National Library

of Canada

Acquisitions and

Bibliographic Servi zos Branch

395 Wellington Streel

Ontawa. Oniaro

KIA ON4
Bibliotheque nationale

du Canada

Direction des acquisitions ef des services bibliographiques

395. ne Wellington

Onawa (Ontario)

KIAON
NOTICE
On the Notreteterence

AVIS
The quality of this microform is heavily dependent upon the quality of the original thesis submitted for microfilming. Every effort has been made to ensure the highest quality of reproduction possible.

If pages are missing, contact the university which granted the degree.

Some pages may have indistinct print especially if the original pages were typed with a poor typewriter ribbon or if the university sent us an inferior photocopy.

Reproduction in full or in part of this microform is governed by the Canadian Copyright Act, R.S.C. 1970, c. C-30, and subsequent amendments.
La qualité de cette microforme dépend grandement de la qualité de la thèse soumise au microfilmage. Nous avons tout fait pour assurer une qualité supérieure de reproduction.

S'il manque des pages, veuillez communiquer avec l'université qui a conféré le grade.

La qualité d'impression de certaines pages peut laisser à désirer, surtout si les pages originales ont été dactylographiées à l'aide d'un ruban usé ou si l'université nous a fait parvenir une photocopie de qualité inférieure.

La reproduction, même partielle, de cette microforme est soumise à la Loi canadienne sur le droit d'auteur, SRC 1970, c. C-30, et ses amendements subséquents. 


\title{
THE SEROTONERGIC AND NORADRENERGIC SYSTEMS OF THE HIPPOCAMPUS: THEIR INTERACTIONS AND THE EFFECTS OF ANTIDEPRESSANT TREATMENTS
}

\author{
Raymond Mongeau \\ Neurobiological Psychiatry Unit and \\ Department of Neurology and Neurosurgery \\ McGill University, Montréal
}

Submitted: August 29, 1994

A Thesis submitted to the Faculty of Graduate Studies and Research in partial fulfilment of the requirements of the degree of Ph.D. in Neuroscience 
National Library

of Canada

Acquisitioris and

Bibliographic Services Branch

395 Wellington Street

Ontawa. Ontario

K1A ON4
Bibliothèque nationale

du Canada

Direction des acquisitions et

des services bibliographiques

395, rue Wellinglon

Ottawa (Ontario)

KIA ON4
Your tite volre rolerence

Qur the Norre rototence
THE AUTHOR HAS GRANTED AN IRREVOCABLE NON-EXCLUSIVE LICENCE ALLOWING THE NATIONAL LIBRARY OF CANADA TO REPRODUCE, LOAN, DISTRIBUTE OR SELL COPIES OF HIS/HER THESIS BY ANY MEANS AND IN ANY FORM OR FORMAT, MAKING THIS THESIS AVAILABLE TO INTERESTED PERSONS.
L'AUTEUR A ACCORDE UNE LICENCE IRREVOCABLE ET NON EXCLUSIVE PERMETTANT A LA BIBLIOTHEQUE NATIONALE DU CANADA DE REPRODUIRE, PRETER, DISTRIBUER OU VENDRE DES COPIES DE SA THESE DE QUELQUE MANIERE ET SOUS QUELQUE FORME QUE CE SOIT POUR METTRE DES EXEMPLAIRES DE CETTE THESE A LA DISPOSITION DES PERSONNE INTERESSEES.
THE AUTHOR RETAINS OWNERSHIP OF THE COPYRIGHT IN HIS/HER THESIS. NEITHER THE THESIS NOR SUBSTANTIAL EXTRACTS FROM IT MAY BE PRNNTED OR OTHERWISE REPRODUCED WITHOUT HIS/HER PERMISSION.
L'AUTEUR CONSERVE LA PROPRIETE DU DROIT D'AUTEUR QUI PROTEGE SA THESE. NI LA THESE NI DES EXTRAITS SUBSTANTIELS DE CELLECI NE DOIVENT ETRE IMPRIMES OU AUTREMENT REPRODUITS SANS SON AUTORISATION.

ISBN $\quad 0-612-05761-5$ 


$$
\begin{gathered}
\text { A ma mire } \\
\text { Laurence Mongeau }
\end{gathered}
$$




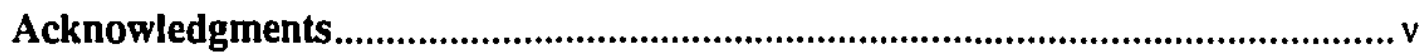

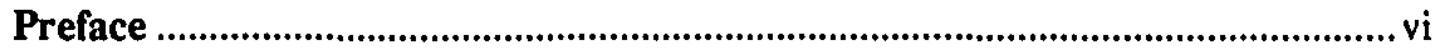

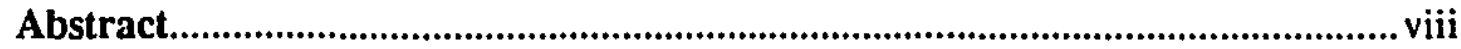

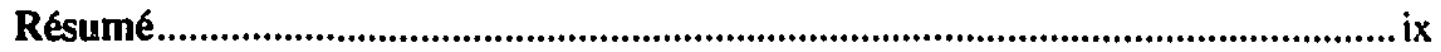

Chapter I - Review of the literature

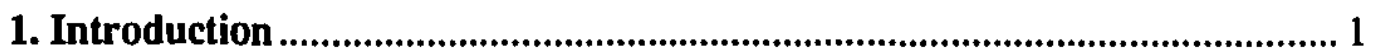

2. Basic concepts

2.1. Serotonin (5-HT) and 5-HT receptors ....................................................... 4

2.2. Noradrenaline (NA) and adrenoceptors ....................................................... 7

3. Physiology of serotonergic and noradrenergic neurons

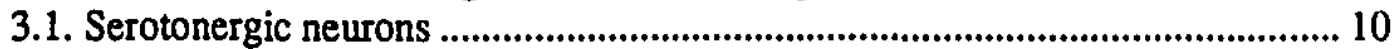

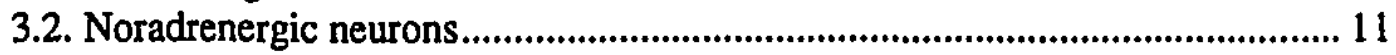

4. Distribution of 5-HT and NA innervations of the hippocampus .................. 13

5. Serotonergic neurotransmission in the hippocampus

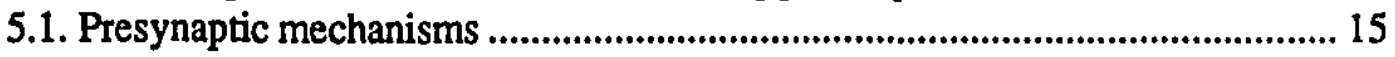

5.2. Postsynaptic mechanisms

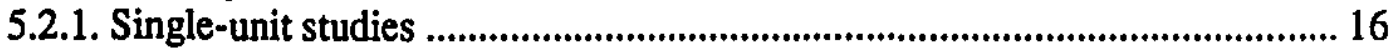

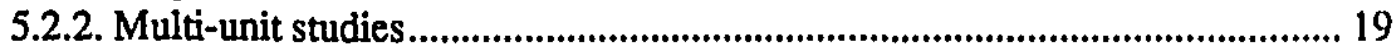

6. Noradrenergic neurotransmission in the hippocampus

6.1. Presynaptic mechanisms ........................................................................ 20

6.2. Postsynaptic mechanisms

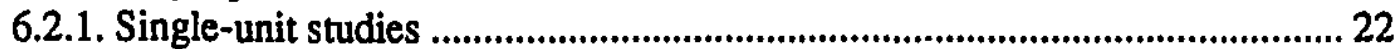

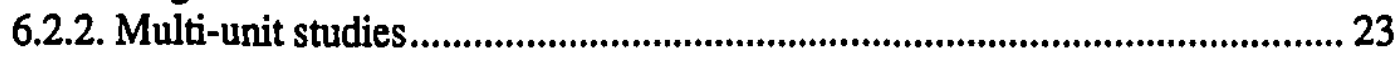

7. Effects of antidepressant treatments on 5-HT and NA neurons

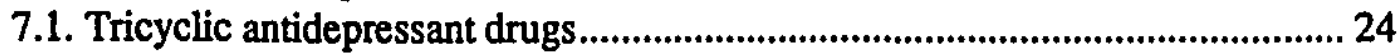

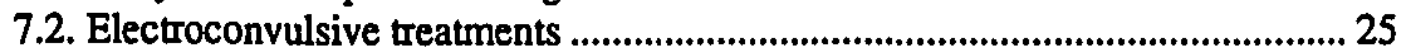

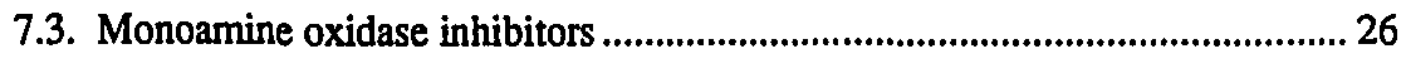

7.4. Selective reuptake blockers....................................................................... 27

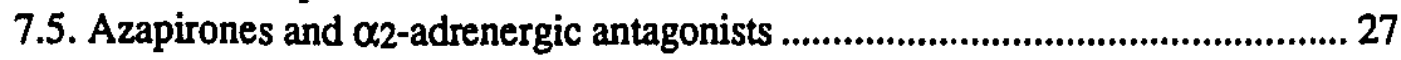

8. Effects of antidepressant treatments on hippocampal 5-HT and NA systems

8.1. At the presynaptic level

8.1.1. Tricyclic antidepressant drugs ..................................................................2. 29

8.1.2. Electroconvulsive treatments ................................................................... 30 


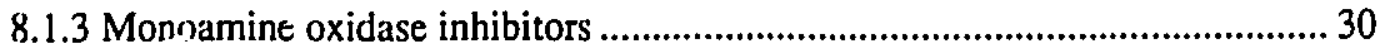

8.1.4 Selective reuptake inhibitors .................................................................... 31

8.1.5 Azapirones and $\alpha_{2}$-adrenergic antagonists .............................................. 32

8.2. At the postsynaptic level

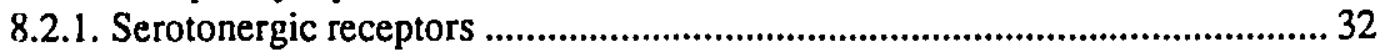

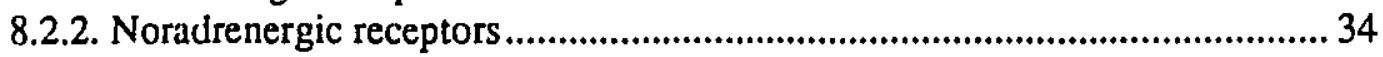

9. Interactions between the serotonergic and noradrenergic systems ............... 36

9.1. Neurochemical evidence for interactions at prisynaptic terminals................... 38

9.2. Neurochemical evidence for interactions at postsynaptic sites ........................ 39

9.3. Behavioral evidence for interactions in the brain............................................ 40

9.4. Evidence for interconnections between 5-HT neurons of the

mesencephalic raphe nuclei and NA neurons of the locus coeruleus....................... 41

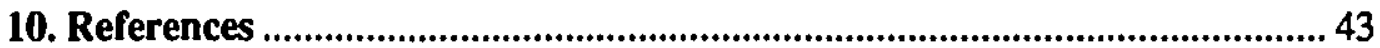

\section{Chapter II - First article}

In vivo electrophysiological evidence for tonic activation by endogenous noradrenaline of $\alpha_{2}$-adrenoceptors on 5-hydroxytryptamine terminals in the rat hippocampus

\section{Chapter III - Second article}

Electrophysiological evidence for desensitization of $\alpha_{2}$-adrenoceptors on serotonin terminals following long-term treatment with drugs increasing norepinephrine synaptic concentration

\section{Chapter IV - Third article}

Effects of long-term $\alpha_{2}$-adrenergic antagonists and electroconvulsive treatments on the

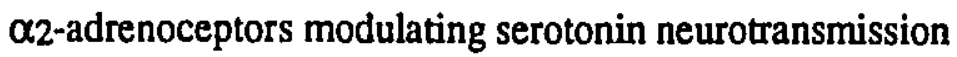

\section{Chapter V - Fourth article}

Activation of 5-HT3 receptors enhances the electrically evoked release of [3H]noradrenaline in rat brain limbic structures

\section{Chapter VI - Fifth article}

Effect of long-term administration of antidepressant drugs on the 5-HT3 receptors that enhance the electrically evoked release of $[3 \mathrm{H}]$ noradrenaline in rat hippocampus

\section{Chapter VII - General discussion}




\section{ACKNOWLEDGEMENTS}

I wish to express my sincere gratitude to Dr Claude de Montigny, Director of the Neurobiological Psychiatry Unit, and Dr. Pierre Blier, Associate Professor at McGill University, for accepting me as a graduate student, supervising my research with competence and providing me with excellent research facilities. I had the opportunity to benefit from a stimulating scientific environment that Claude and Pierre generate by their exceptional energy and enthusiasm to elucidate neurophysiological processes. I would also like to thank all research students, fellows, technicians and secretaries whose help and friendship made this period of my life enjoyable.

I would like to thank in advance the members of the Thesis committee for contributing their time and effort to the evaluation of this lengthy document.

This work was made possible by the financial support of the Fonds de Recharche en Santé du Québec and the Medical Research Council of Canada. 


\section{Preface}

The studies and the reviews of the present thesis concern the serotonergic and the noradrenergic systems of the hippocampus, their interactions, and their implications in the mechanism of action of antidepressant treatments. I have chosen the option provided in the "Guidelines Concerning Thesis Preparation" which allows inclusion as chapters of the thesis the text of original papers concerning the thesis research project. The Faculty regulations required to be cited in full are the following:

"Candidates have the option, subject to the approval of their Department, of including, as part of their thesis, copies of the text of a paper(s) submitted for publication, or the clearly-duplicated text of a pliblished paper(s), provided that these copies are bound as an integral part of the thesis.

- If this option is chosen, connecting texts, providing logical bridges between the different papers, are mandatory.

- The thesis must still conform to all other requirements of the "Guidelines Concerning Thesis Preparation" and should be in a literary form that is more than a mere collection of manuscripts published or to be published. The thesis must include, as separate chapters or sections: (1) a Table of contents, (2) a general abstract in English and French, (3) an introduction which clearly states the rationale and objectives of the study, (4) a comprehensive general review of the background literature to the subject of the thesis, when this review is appropriate, and (5) a final overall conclusion and/or summary.

- Additional material (procedural and design data, as well as descriptions of equipment used) must be provided where appropriate and in sufficient detail (e.g. in appendices) to allow a clear and precise judgement to be made of the importance and originality of the research reported in the thesis.

- In the case of manuscripts coauthored by the candidate and others, the candidate is required to make an explicit statement in the thesis of who contributed to such work and to what extent; supervisors must attest to the accuracy of such claims at the Ph.D. Oral defense. Since the task of the examiners is made more difficult in these cases, it is the candidate's interest to make perfectly clear the responsibilities of the different authors of coauthored papers."

The experimental parts of the thesis (chapters 2, 3, 4, 5 and 6) consist of four original articles that have been already published and one submitted for publication. In these five studies, I have done all the experimental work, written the manuscripts, performed the calculations and statistical analysis and drawn the figures. The use of the terms "we", and "our" within the text refers to myself and my thesis supervisors, Drs. P. Blier and C. de Montigny. Together with my thesis supervisors, I have contributed to the 
design of the experiments, to the formulation of the working hypothesis and to the interpretation of the results. A comprehensive general review of the literature is provided in chapter 1. The overall summary and discussion of the different studies of this thesis are made in chapter 7 .

"- Elements in the thesis that are to be considered as contributions to original knowledge must be clearly indicated in the preface or by separate statement at the beginning or at the end of the thesis. This requirement is mandatory for Ph.D. theses.

- Theses should also contain an appropriate, not necessarily long or exhaustive, historical statement of previously relevant work or investigation."

The existence of $\alpha_{2}$-adrenergic heteroreceptors that inhibit the release of serotonin has been known since the early 80's from in vitro studies. The work presented in chapter 2 is the first in vivo electrophysiological characterization of these heteroreceptors. There were some clues in the literature suggesting that the 5-HT3 agonist 2-methyl-5-HT modulates the release of noradrenaline. The study presented in chapter 5 demonstrates that there are 5-HT3 receptors that enhance the release of noradrenaline in rat brain limbic areas. The other studies of this thesis are original contributions on the effect of antidepressant drugs at the level of the serotonergic and noradrenergic systems, except the finding that repeated electroconvulsive shock increases the serotonergic neurotransmission (chapter 5) which was already reported by de Montigny and collaborators. We were the first to provide evidence that $\alpha_{2}$-adrenergic heteroreceptors become desensitized following long-term treatments with drugs that increase the synaptic concentration of noradrenaline (chapters 4 and 5). The last study of this thesis (chapter 6) investigates for the first time the effect of antidepressant drug treatments on serotonergic receptors that modulate the release of noradrenaline. Finally, the review of the literature of chapter 1 is also a contribution to original knowledge since no reviews on serotonergic and noradrenergic neurotransmission and the effects of antidepressant treatments have ever focused specifically on the hippocampus. Furthermore, as far as I know, the review provided in this chapter on the interactions between the central serotonergic and noradrenergic systems of the brain has never been done before. 


\begin{abstract}
Since the formulation of the monoaminergic hypotheses of depression, several investigations have established that the serotonin (5-HT) as well as the noradrenaline (NA) systems are altered by antidepressant treatments. In the last few years, several studies have indicated that interactions between these two systems might also be important in the mechanism of action of antidepressant drugs. We have thus undertaken: 1) to elucidate the nature of some interactions between the 5-HT and NA systems in the rat hippocampus using in vivo electrophysiology and in vitro superfusion techniques; and 2) to determine whether antidepressant treatments alter these interactions. It was found that NA tonically inhibits 5-HT neurotransmission via $\alpha_{2}$-adrenergic heteroreceptors. In addition, the presence of $5-\mathrm{HT}_{3}$ receptors facilitating the release of NA was also documented in this brain region. These receptors were shown to be activated by elevated levels of endogenous 5-HT, but did not appear to be activated by basal levels of this neurotransmitter. Long-term administrations of antidepressant drugs commonly known to increase the synaptic concentration of NA, i.e. monoamine oxidase and NA reuptake inhibitors, were found to desensitize the $\alpha_{2}$-adrenoceptors that inhibit the release of 5HT. This effect was also produced by atypical antidepressant drugs that have $\alpha_{2}$ adrenergic antagonist properties (e.g. mianserin and idazoxan), but not by other antidepressant treatments, (e.g. selective 5-HT reuptake inhibitors or electroconvulsive shocks). In contrast to $\alpha_{2}$-adrenergic heteroreceptors, 5-HT3 receptors that enhance the release of NA did not become desensitized following long-term treatments with drugs that elevate the synaptic concentration of their endogenous neurotransmitter. Rather, the responsiveness of these 5-HT3 receptors became blunted following an antidepressant treatment (desipramine) that caused a large increase in the release of NA. Taken together, these results suggest that presynaptic receptors mediating interactions between the NA and 5-HT systems might exert an important role in the mechanism of action of antidepressant treatments.
\end{abstract}




\section{Résumé}

Depuis la formulation des hypothèses monoaminergiques de la dépression, plusieurs études ont clairement établit que les systèmes sérotoninergiques et noradrénergiques sont affectés par des traiterrents antidépresseurs. Au cours des dernières années, plusieurs études ont indiqué que les interactions entre ces deux systèmes sont importantes dans les mécanismes d'action des antidépresseurs. Nous avons donc entrepris d'élucider, premièrement, la nature des interactions entre les systèmes sérotoninergique et noradrénergique dans l'hippocampe du rat en utilisant des techniques électrophysiologiques in vivo et de superfusion in vitro. Deuxièmement, nous avons déterminé si des traitments antidépresseurs reconnus altèraient ces interactions. Il fut établit que la noradrénaline (NA) inhibe toniquement la neurotransmission sérotoninergique par l'intermédiaire des hétérorécepteurs $\alpha_{2}$-adrénergiques. De plus, dans cette région du cerveau, la présence de récepteurs du type 5-HT3 facilitant la libération de la NA fut également établit. Nous avons montré que ces derniers sont activés par des niveaux élevés de sérotonine (5-HT) endogène, mais non par des niveaux de base de ce neurotransmetteur. Les traitements à long-terme avec des antidépresseurs tels que les inhibiteurs de la monoamine oxidase ou de la recapture de la NA ayant la propriété d'augmenter la concentration synaptique de la NA, ont désensibilisé les adrénorécepteurs de type $\alpha 2$ qui inhibent la libération de la 5-HT. Ce dernier effet a aussi été produit par des traitements antidépresseurs atypiques ayant des propriétes antagonistes $\alpha_{2}-$ adrenergiques (mianserin and idazoxan), mais non par d'autres traitment antidépresseurs, tel qu'un inhibiteur sélectif de la recapture de la 5-HT ou les traitements électroconvulsifs. Contrairement aux hettérorécepteurs $\alpha 2$-adrénergiques, les récepteurs 5-HT3, qui augmentent la libération de la NA, n'ont pas été désensibilisés par des traitements à long terme avec des médicaments qui augmentent la concentration synaptique de leur neurotransmetteur. La sensibilité de ces récepteurs 5-HT3 s'est plutôt vu réduite suite à un traitement (désipramine) qui a entrainé une grande augmentation de la libération de la NA. Dans l'ensemble, ces résultats suggèrent que les récepteurs présynaptiques qui médient les interactions entre les systèmes 5-HT et NA sont des éléments neuronaux importants impliqués dans le mode d'action des médicaments antidépresseurs. 


\section{REVIEW OF THE LITERATURE}

\section{Introduction}

The present research endeavour is aimed at better understanding the interactions between brain neuronal systems implicated in the regulation of mood and the biological basis of pharmacological treatments currently used in the treatment of depression. Depression is a mental illness with both psychological and biological symptoms. Asdescribed in the Diagnostic and Statistical Manual of Mental Disorders (DSM IV) ${ }^{1}$, major depressive episodes are characterized by depressed mood, attenuated interest (anhedonia), feelings of worthlessness, anorexia or hyperphagia, insomnia or hypersomnia, and recurrent thoughts about death or suicide. At the turn of the century, Kraepelin made a fundamental distinction between endogenous (somatic, physiological, biochennical) and exogenous (reactive) depressive disorders. Although the boundary between these two conditions may appear rather fuzzy, it remains that some depressions seem to be reactions to psychological or environmental stressful life events, whereas others have no obvious psychogenic causes and mightrather be the consequence of some primary biological defect in the brain. Genetic factors are thought to be important. The mode of transmission is described as multifactorial inheritance because the inherited depressive genotypes would interact with environmental factors to produce the disease ${ }^{161}$. DSM IV makes distinctions between various types of affective disorders. For instance, major depression is diagnosed when severe symptoms of depression are present nearly every day for at least two weeks, whereas dysthymia is characterized by depressive symptoms present for two years but not of sufficient severity and duration to meet the criteria of a major depressive episode. It is noteworthy that depression is often associated with other mental disorders. In bipolar depression, the person experiences manic episodes (elevated mood, hyperactivity, flight of ideas, megalomania, hyposomnia, ...) that alternate with depressive episodes. Patients with Alzheimer's disease, who suffer brain degeneration in some areas such as the hippocampus, experience loss of memory functions and also often have secondary symptoms of depression in greater proportion than patients with other afflicting diseases. It is also noteworthy that in most instances there is a significant degree of anxiety in depressed patients. Depression may occur secondary to a generalized anxiety disorder or a panic disorder ${ }^{147}$, and classical antidepressantdrugs are effective against these two an:iety disorders 243,244 .

It is important to point out that despite intensive research, the etiological factors leading to depression remain unknown. According to the Cognitive Theory of Depression 35 , depressive individuals tend to distort events and draw erroneous conclusions leading to self-blame. Traumatic events during early youth would be responsible for the development of inadequate attitudes and depression. Other authors have subsequently linked the concept of learned helplessness to depression (Cognitive-Learning Theory) 253,400 . According to this theory, anxiety would be the initial reaction of an individual to stressful or frustrating situations. In some cases when aversive events are not resolved, individuals might develop the conviction that they cannot control recurrent stress or that they have no hope to ever obtain gratification and secondarily adopt depressive behaviors as maladaptive defense mechanisms. Similarly, 
when animals are exposed to uncontrollable aversive events, such as electric shocks, they typically develop behavioral deficits. They will fail to escape subsequent electric shocks even though they have the possibility. Furthermore, their performances in rewarded behavior conditions are consistently impaired, suggesting that they are afflicted with anhedonia. Memory functions are thought to play a central role in learned helplessness. The difference between individuals who become depressed and those who adequately overcome stressful experience might be related to their resilient attitude, or in other words, with the capacity of their brain to disrupt, disconnect or disengage previously learned associations if these now lead to an aversive outcome ${ }^{114}$.

One brain area of interest in relation to memory functions and depression is the hippocampus. Gray has provided an impressive review suggesting that this structure is implicated in short-term memory and could serve the function of a comparator that would compare actual versus expected stimuli ${ }^{179}$. This hippocampal comparator would be particularly useful to detect anxiogenic stimuli and activate appropriate behaviors in reaction to them ${ }^{179}$. There is evidence indicating that the behavioral deficits of learned helplessness can be prevented by hippocampal lesions ${ }^{128}$. In addition, the hippocampus would be a central site implicated in the negative feedback on the secretion of glucocorticoids released during stress ${ }^{378}$. Hypercortisolism has been consistently observed in depressed patients and recent data indicate that this might be related to an abnormal feedback mechanism occurring at a central site such as the hippocampus, rather than at the pituitary 479 . In animal models of depression, chronic stress inhibits fast glucocorticoid negative feedback and down-regulates glucocorticoid receptors selectively in the hippocampus 478 .

Since the formulation of the indoleamine hypothesis of depression, suggesting that serotonin (5HT) is decreased in depressed patients ${ }^{262}$, many studies have supported this but did not yield unequivocal evidence of decreased 5-HT neurotransmission in depression ${ }^{115}$. Here are some findings supporting that hypothesis. Selective reduction of hippocampal 5-HT1A receptors has been found in the brain of depressed patients ${ }^{92}$, and high levels of glucocorticoids decrease the density of 5-HT1A receptors in the rat hippocampus ${ }^{301}$. Interestingly, activation of 5-HT1A receptors reversed the behavioral deficits of learned helplessness in rats ${ }^{176}$. Furthermore, inhibition of 5-HT synthesis with para-chlorophenylalanine produced relapse of depression in patients who had responded to antidepressant drugs 405.406 . Deakin has reviewed evidence suggesting that the 5-HT1A neurotransmission of the hippocampus might be involved in disengaging behaviors that lead to aversive outcomes. According to this hypothesis, the serotonergic system of the hippocampus mediates tolerance to chronic aversive events. Thereby, an increase in 5-HT neurotransmission would be a natural antidepressant ${ }^{114}$.

The early catecholamine hypothesis of depression proposed that the symptoms of this disease are linked with a deficiency of noradrenaline (NA) in the brain ${ }^{383}$. In contrast, other authors have suggested that NA might actually be increased in depression 250,256 . An integrated view has also been proposed whereby, rather than being either reduced or enhanced, NA neurotransmission would be dysregulated 407 . Similarly to what has been found in animal models of learned helplessness, the basal output of NA might 
be decreased in depression due to a diminution of this neurotransmitter in the cytosolic stores ${ }^{469}$. However, this NA depletion would diminish the inhibitory feedback mechanisms controlling, for instance, NA synthc sis or NA neurons' firing activity. Consequent to this decreased NA autoinhibition, depressive patients would respond to acute stressors with enhanced noradrenergic activity 407 . There are also several lines of evidence indicating that the NA system of the hippocampus would play an important role in learned helplessness. An up-regulation of $\beta$-adrenergic receptors confined to the hippocampus of animals exhibiting behavioral deficits following the learned helplessness training has been reported 240 , and direct injection of an antidepressant drug into the hippocampus prevented these behavioral deficits ${ }^{403}$. Moreover, selective destruction of the noradrenergic fibers of the hippocampus delayed the reversal of learned helplessness by long-term antidepressant drug treatments ${ }^{411}$. Gray ${ }^{179}$ has also reviewed evidence indicating that the NA system, by its arousing and memory-enhancing functions, would engage the septo-hippocampal system in the processing of anxiogenic stimuli. It is also important to point out that the NA neurotransmission in the hippocampus and circulating glucocorticoids mutually interact $237,238,249$.

Perhaps the most convincing evidence indicating that the serotonergic and noradrenergic systems of the brain are relevant to the therapy of depression is the fact that most clinically effective drugs used for the treatment of this disease act directly on the synaptic concentration of 5-HT and or NA. The first drug commercialized in the late 1950's for the treatment of depression was iproniazid, an inhibitor of the enzyme monoamine oxidase (MAO). The concentrations of 5-HT and NA are increased in the brain by blocking their degradation by MAO. Since this original discovery, many other MAO inhibitors (e.g., phenelzine, tranylcypromine, clorgyline, moclobemide, ...) have been shown to be clinically effective for the treatment of depression. Imipramine was also tested during the same period for its potential as an antipsychotic drug, but it turned out that imipramine was effective in depression rather than in schizophrenia. This tricyclic antidepressant drug as well as others of this class (e.g., amitryptiline, desipramine, chlorimipramine) were shown to share the property of blocking the reuptake of NA and 5-HT with different potencies, and their therapeutic efficacy was thereby thought to be related to the prolongation of the synaptic action of these neurotransmitters ${ }^{470}$. Subsequently, selective inhibitors of either 5-HT reuptake (e.g., citalopram, fluoxetine, paroxetine) or NA reuptake (e.g., maprotiline, oxaprotiline, amoxapine) were shown to display antidepressant efficacy as well 373,404 . Some antidepressant drugs were found to act directly at 5-HT or adrenergic receptors. For example, mianserin and trazodone increase NA output by blocking $\alpha 2$-adrenergic autoreceptors ${ }^{470}$. Furthermore, antidepressant drugs of the azapirone family (e.g., buspirone, gepirone) act as partial agonists at the level of the 5-HT $1 \mathrm{~A}$ receptors ${ }^{367}$. It is noteworthy that aithough the above effects on the noradrenergic and serotonergic systems are observed following acute administration, long-term treatments are required for all these drugs in order to achieve clinical efficacy.

It was proposed that there might be two types of depressions, known as $A$ and $B$, linked respectively with decreases in NA and 5-HT neurotransmission 276. In type A depression, patients would have low pretreatment levels of NA metabolites (e.g. MHPG) and this parameter would normalize with clinical 
improvement. Furthermore, these patients would have rapid elevation of mood when administered a NA releaser (amphetamine) and exhibit a good antidepressant response to NA reuptake blockers. On the other hand, in type B depression, patients would have low pretreatment levels of 5-HT metabolites (e.g. 5-HIAA), a lack of response to amphetamine and a favourable response to 5-HT reuptake blockers. However, this hypothesis was not supported by clinical findings, and antidepressant agents inhibiting either 5-HT or NA reuptake are equally effective and are not selective for subtypes of depression 477 .

In the next sections, one of my aims is to review the most fundamental elements of serotonergic and noradrenergic neurotransmissions. Several elements of the 5-HT and NA systems (synthesis, receptors, second messengers, ...) are discussed here, all being potential targets for antidepressant treatments. In the second part of this review, the issue of the effects of antidepressant treatments on the 5-HT and NA systems is directly addressed. I have decided to focus mainly on the hippocampus as my research project is mainly concerned with this area. In the third and last part, I review data from various experiments indicating that the 5-HT and NA systems interact extensively in the brain.

\section{Basic concepts}

\subsection{Serotonin and 5-HT receptors}

Long before its discovery in the brain, physiologists knew that serotonin (5-hydroxytryptamine, 5-HT) is a vasoconstrictor agent found in serum when blood is allowed to clot ${ }^{16}$. The findings that lysergic acid diethylamide (LSD), a potent hallucinogen, shares structural similarities with 5-HT and blocks some peripheral responses to 5-HT opened an importantchapter in neuropsychopharmacology 155 . Furthermore, the notion that reserpine, a potent tranquilizing drug used at the time, produces a depletion of 5-HT in the brain sometimes correlating with behavioral "depression" was another important landmark 75 . These early studies already suggested that 5 -HT could be a neurotransmitter.

This function is now well established, and the principal biochemical steps surrounding the neuronal synthesis and degradation of 5-HT are known. The limiting precursor of 5-HT synthesis is an amino acid, tryptophan, which arises primarily from the diet and crosses the blood-brain barrier through a non-specific carrier for large neutral aminoacids ${ }^{99}$. As shown in the figure 1, tryptophan hydroxylase adds a hydroxyl group at the position 5 of the indole ring of tryptophan to yield 5-hydroxytrytophan (5HTP). A non-specific enzyme decarboxylates of either tryptophan or 5-HTP which then become tryptamine or 5-hydroxytryptamine (5-HT), respectively305.

The degradation of 5-HT into its major metabolite 5-hydroxyindoleacetic acid (5-HIAA) is carried out in the brain through the action of the monoamine oxidase (MAO) and aldehyde dehydrogenase (Fig. 1). Although the MAO present in 5-HT neurons is of type B, this enzyme degrades 5-HT only when the concentration of this neurotransmitter is elevated since MAOB has a very low affinity for 5-HT. In fact, extraneuronal 5-HT is mostly degraded by the MAO of type A localized in cathecolaminergic neurons 267,464 . However, the removal of 5-HT from the synaptic cleft is affected primarily by its reuptake into serotonergic terminals via a specific carrier, rather than by the action of MAO $A^{151}$. 

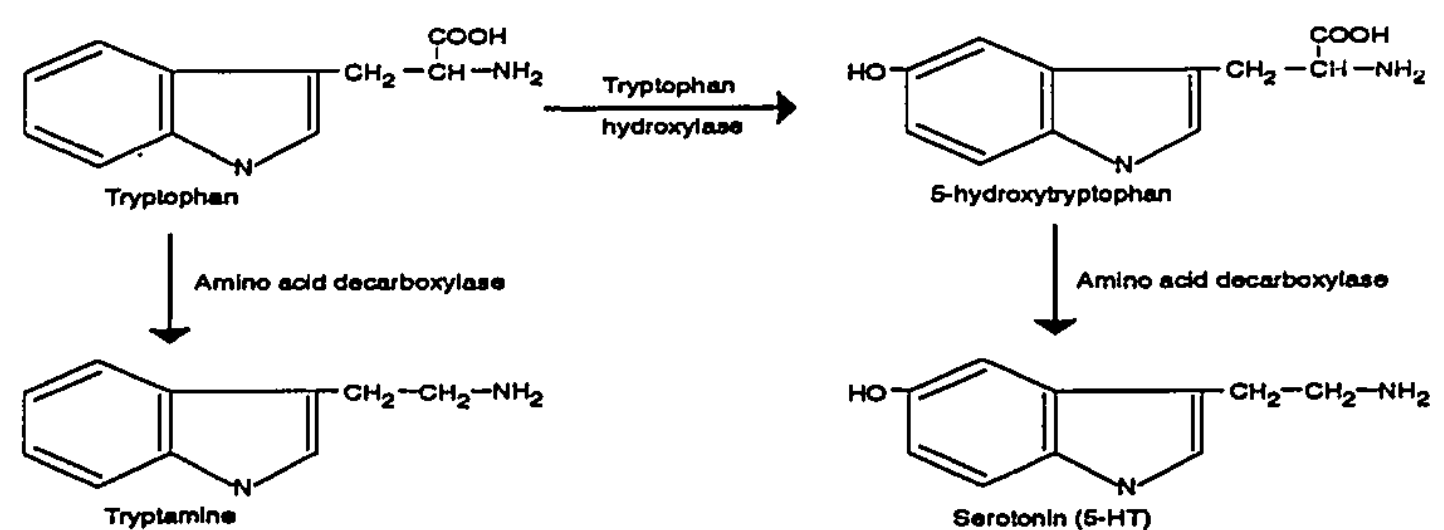

6-hydroxytryptophan

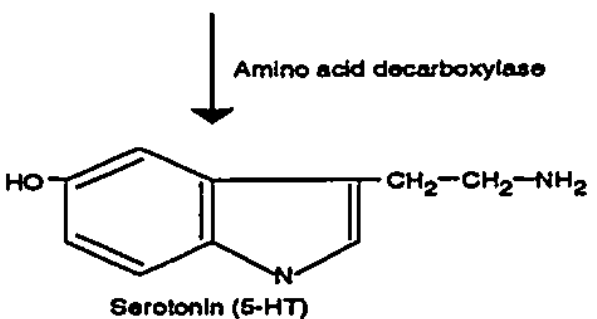

Fig. 1. 5-HT synthesis and metabolism

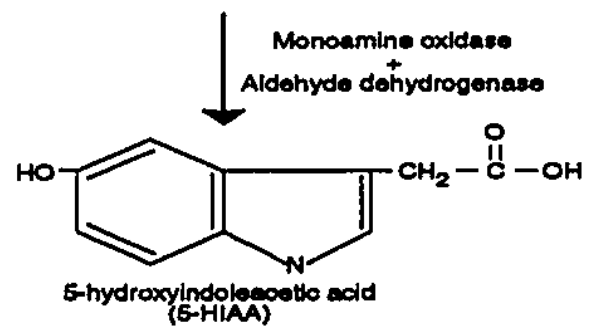

Most 5-HT-containing neurons are clustered in or near the midline region of the caudal mesencephalon, the pons and upper brain stem in a region denominated the raphe area ${ }^{154}$. The more caudal groups of the brainstem project mostly to the medulla and the spinal cord. Among the more rostral groups are the nuclei designated as the dorsal raphe (DR) and the median raphe (MR) that provide extensive 5-HT innervation of the telencephalon and diencephalon. The fibers of the DR and the MR course together through the ventral tegmentum and into the medial forebrain bundle before projecting to differential sites. The main projections of the DR are the brain cortex, the striatum, the amygdala, the nucleus accumbens, the lateral septum and the hypothalamus. The hippocampus receives innervation from the DR but also from the MR. Apart from the hippocampus, the latter nucleus has important terminal sites in the frontal cortex, the septum and mesolimbic areas26,98,263,284,437.

There is considerable evidence for heterogeneity in receptor types involved in 5-HT neurotransmission. Remarkable progress has been made these last few years regarding the classification of 5-HT receptors in the brain. The distinction between 5-HT 1 and 5-HT2 receptors, commonly accepted since the early 80 's, is based on the respective affinities of these receptors for [3H]5-HT and related agonists (5-HT1 $>5-\mathrm{HT}_{2}$ ) and for certain 5-HT antagonists such as [3H] spiperone (5-HT2 $\left.>5-\mathrm{HT}_{1}\right)$. The nature of the transduction mechanisms linked to these receptors is another criterion generally accepted to differentiate 5-HT receptors. 5-HT1 receptors inhibit adenylate cyclase through $\mathrm{G}_{\mathrm{i}}$ proteins while the 5-HT2 receptors stimulate phosphoinositide metabolism through $G_{p}$ proteins 196,226 .

The 5-HT1 receptor class in the brain has been further subdivided into the 5-HT1A, 5-HT1B, 5HTIC $_{1 \mathrm{C}}$ 5-HT1D receptors ${ }^{347}$. The relatively selective ligand 8-OH-DPAT (8-hydroxy-2-[di-Npropylamino]-tetralin) has been invaluable for the characterization of the 5-HT1A receptor subclass ${ }^{188,346}$. Many agonists such as 5-carboxyamidotryptamine (5-CT) or buspirone, and classical 5HT antagonists such as spiperone or LSD, as well as $\beta$-adrenergic antagonists such as (-)pindolol or ()propanolol, have been shown to display high affinity for this receptor in competition studies. 
Somatodendritic autoreceptors on 5-HT neurons of various species, including humans, are thought to be of the 5-HTIA subtype as the high density binding of [3H]8-OH-DPAT in the raphe area is markedly reduced following lesions of 5-HT neurons $222,451,462$. In the hippocampus, the binding of $[3 \mathrm{H}] 8-\mathrm{OH}-$ DPAT is also very dense, and these 5-HT 1 A receptors would be localized on postsynaptic cells rather than on 5-HT terminals ${ }^{188,248,451}$. Extended saturation analysis of [3H]8-OH-DPAT binding in the rat hippocampus revealed that the ligand would discriminate between high and low affinity states of the 5 . HT 1 A receptor. Further studies suggested that the high affinity site corresponds to a state stabilized with a regulatory G-protein, whereas the low affinity site corresponds to the uncoupled state of the receptor 313 . Cloning and functional expression of the mRNA coding for the 5-HT1A receptor were realized, and the pharmacological characteristics of the cloned receptor were similar to those found for the native $5-\mathrm{HT}_{1 \mathrm{~A}}$ receptor of the brain 10 .

The rat hippocampus has also been shown to be endowed with 5-HTIB receptors using $[3 \mathrm{H}]$ dihydroergotamine or $(-)[125\rceil$ iodocyanopindol ${ }^{189,221,308}$. These radioligands, used in conjunction with unlabeled adrenergic ligands and 8-OH-DPAT to achieve receptor selectivity, label a single population of sites 189,221 . The 5-HT1B receptors resemble 5-HT1A receptors since agonists such as 5$\mathrm{CT}$, or antagonists, such as methiothepin, display good affinities for these sites. However, 5-HT $1 \mathrm{~B}$ receptors are distinguished from the 5-HT1A subtype by their very low affinities for ligands such as buspirone and spiperone. Functional characterization revealed that terminal autoreceptors regulating 5HT release are of the 5-HT $1 B$ subtype in the rat brain 131,210,295. However, it appears that most 5-HT $1 B$ binding receptors in regions such as the rat hippocampus are postsynaptic because these sites are either unaltered or only slightly reduced by selective lesions of 5-HT neurons ${ }^{145,450}$.

There is no $(-)[125]$ iodocyanopindol binding in human brains nor in other species such as the guinea-pig222. Various studies suggest that 5-HT1D rather than 5-HT1B receptors serve terminal autoreceptor functions in humans and guinea-pigs brains 223 . The conclusion of these studies lies in the fact that 5 -HT 1 D receptors have a pharmacological profile distinct from that of 5-HT1B receptors, mainly with regard to their affinities for $\beta$-adrenergic ligands. It is noteworthy that two types of human $5-\mathrm{HT}_{1 \mathrm{D}}$ receptors have been cloned, the 5-HT1D $\alpha$ and the 5-HT1Dß. The 5-HTID $\beta$ receptor has a high genomic sequence homology with the rodent 5-HT1B receptor 226,236 . Recent studies indicated that mRNAs coding for the 5-HT $1 \mathrm{D} \alpha$ receptor are present in the rat brain 190 and electrophysiological data sugge: : that 5-HT1D $\alpha$ receptors modulate the release of 5-HT in the rat dorsal raphe area352.

Most 5-HT2 agonists such as $\alpha$-methyl-5-HT and antagonists such as ritanserin are also acting, at 5-HT1Creceptors ${ }^{269}$. Furthermore, 5-HT1C receptors share with 5-HT2 receptors a similar transduction system and amino acid sequence homology 196,220. The Serotonin Club Receptor Nomenclature Committee has thus recently decided to change the name of the 5-HT1C to the 5-HT2 receptor and the classical 5-HT2 to the 5-HT2A receptor ${ }^{226}$. These two receptors can be distinguished by their relative affinity for certain antagonists. For instance, small concentrations of [3H]ketanserin or [3H]spiperone mainly label 5-HT2A receptors and cisapride has a nanomolar range affinity for the 5-HT2A but not for the 5-HT2C receptors 269 . 
Recently, a third class of 5-HT recognition sites, the 5-HT3 receptors, has been characterized in different species and tissues 70,364 . The 5-HT3 receptor does not have the seven transmembrane domains linked with $\mathrm{G}$ protein transduction system, as is the case for 5-HT1 and 5-HT2 receptors, but is rather a member of the ion channel-gated family, which includes the nicotinic receptors 220 . Although 2methyl-5-HT and phenylbiguanide are prototypic agonists for 5-HT3 receptors, their affinities for these receptors are relatively low, but nevertheless comparable to that of 5- $\mathrm{HT}^{219}$. Advances in the pharmacological characterization of 5-HT3 receptors are mainly attributable to the development of new ligands such as ondansetron, tropisetron and zacopride that are highly pot'nt antagonists at these sites 220 . A widespread distribution of binding sites for these ligands is found centrally as well as peripherally. By using the 5-HT3 antagonist, [3H]GR65630, autoradiographic analysis ${ }^{247}$ showed high density of 5HT3 sites in various regions of the rat brain, such as the hippocampus, the septurn and the area postrema. It is noteworthy that subunits of the 5-HT3 receptor have been cloned, and the expressed receptor has pharmacological properties similar to that of the native receptor found in the brain 239,288 .

The latest receptor class officially accepted into the 5-HT receptor family is the 5-HT4 subtype ${ }^{226}$, which has recently been cloned 160 . Cisapride and 5-methoxytryptamine are agonists with high affinities for these sites, whereas 2-methyl-5-HT is inactive. Some 5-HT3 receptor antagonists such as tropisetron and ondansetron are active as antagonist at 5-HT4 receptors, albeit they have a lower affinity at these sites. In contrast, the 5-HT3 antagonist zacopride acts as an agonist at 5-HT4 receptors 219,220 . The complete characterization of binding at 5-HT4 receptors has been done recently using [3H]GR113808, and saturation analysis revealed a single site of high affinity in brain areas such as the hippocampus ${ }^{184}$. The highest densities of these receptors are found in the striatum, substantia nigra and olfactory tubercule ${ }^{184}$. Finally, it is noteworthy that other 5-HT receptor subtypes denominated 5-HT1E/1F and $5-\mathrm{HT} 5 / 6 / 7$ have also been recently proposed based on molecular cloning and pharmacological data 66 .

\subsection{Noradrewaline and adrenoceptors}

Noradrenaline (NA; also known as norepinephrine), and its structural analogues adrenaline and dopamine, belongs to a class of hormones and neurotransmitters known as the catecholamines. The first proposal of neurohormonal transmission came at the beginning of the century 260,268 from experiments using extracts of adrenal glands containing adrenaline. The early studies of Cannon and coworkers 82 reported a substance, released from the liver upon sympathetic nerve stimulation, closely resembling adrenaline but having different effects on heart vessels. This substance was subsequently identified in 1946 as being NA, and was shown to be released by sympathetic nerve terminals ${ }^{135}$. For some years after, there was controversy concerning whether the presence of NA in the brain reflectcd only the sympathetic innervation of cerebral blood vessels. This issue was resolved in 1954 by Vogt who showed that NA distribution in the brain is not uniform and does not coincide with the density of blood vessels found in any given brain region, suggesting rather that NA would serve the function of a central neurotransmitter 99 . 
Tyrosine is the primary precursor of NA and of all catecholamines of the brain. This amino acid is the hydroxylated product of phenylalanine that is assimilated through the diet. Before being utilized by NA neurons, tyrosine is taken up from the bloodstream and actively transported into the brain. The biochemical steps involved in the transformation of tyrosine into NA are summarized below and schematized in figure 2 .

First, a second hydroxyl group is added by tyrosine nydroxylase to the aromatic ring to form the cathecol nucleus of the molecule that becomes DOPA (3,4dihydroxyphenylalanine), the carboxylated precursor of dopamine. The latter (being itself a neurotransmitter in some brain regions) is taken up from the cytoplasm into vesicles, where it then becomes the substrate of dopamine- $\beta$ hydroxylase. This enzyme yields NA by adding a hydroxyl group on the carbon at the $\beta$ position of the side chain 305 .

The major metabolite of NA in the brain is MHPG (3methoxy-4-hydroxyphenylglycol). After its depolarizationevoked release, NA is taken up into noradrenergic terminals by a specific reuptake carrier, where it is then catabolized by the type A MAO. With the help of an aldehyde reductase, MAO A catalyzes the formation of an alcohol that is then transformed into DHPG (3,4-dihydroxyphenylglycol). DHPG is subsequently excreted and becomes the target of the catecholO-methyl transferase (COMT) which transfers a methyl group, taken from S-adenosylmethionine, onto the hydroxyl group at the meta position of the phenyl ring, thereby yielding MHPG (Fig. 2) 305.

The major cluster of NA-containing neurons that innervates the telencephalon and diencephalon is the locuscoeruleus (LC) nucleus, a structure that lies in the dorsolateral part of the pontine tegmentum. There is also a large number of NA neurons that forma more diffuse butcontinuous group thatlies throughout the lateral ventral tegmental field. Apart from the LC, the dorsal aspect of the tegmental area contains another group of NA neurons centered in the dorsal motor nucleus of the vagus ${ }^{263}$. The axons of these NA neurons are intermingled into three noradrenergic bundles: 1 ) The fibers of the dorsal NA bundle (DNB) originating from the LC innervates the whole neocortex, hippocampus and amygdala; 2) The ventral

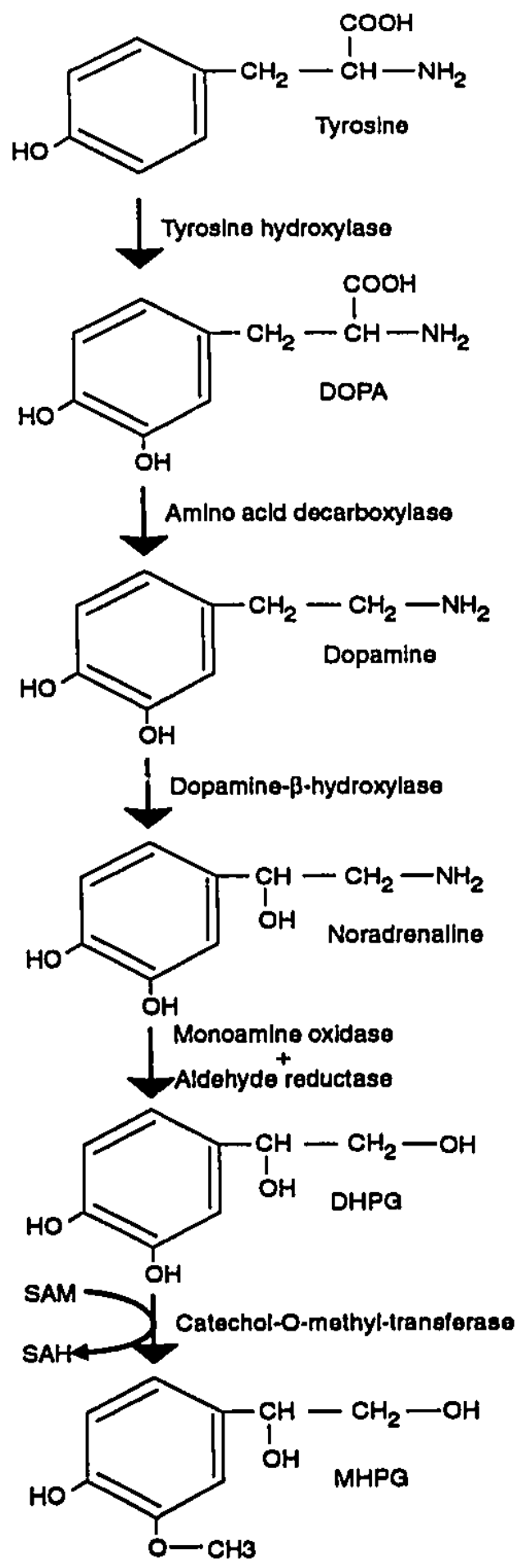

Fig. 2. NA synthesis and metabolism 
NA bundle (VNB) collects fibers from many nuclei of the lateral ventral tegmental group as well as some fibers of the LC, and projects mainly to hypothalamic and mesolimbic structures. In addition, most of the NA fibers that innervate the spinal cord arise from this bundle; 3 ) There is finally the periventricular system (PVS), that originates from the NA neurons centered in the motor nucleus of the vagus, gains fibers from LC and terminates in the central gray or periventricular areas 272 .

The actual classification of adrenergic receptors in the central nervous system relies on the classical pharmacology of three major adrenergic agents acting in the periphery: adrenaline, NA and isoproterenol. Following in vivo and in vitro studies of the metabolic effects of adrenergic agents, Ahlquist proposed a division of their pharmacological responses into two general classes, termed $\alpha$ - and $\beta$-adrenergic ${ }^{9}$. In the brain, as well as in the periphery, NA is more potent than isoproterenol at $\alpha$-adrenoceptors. In contrast, isoproterenol is more potent than NA at $\beta$-adrenoceptors. Since this original definition, development of more selective ligands allowed further subdivision of the two adrenoceptor families into four classes that are found in the brain, termed $\alpha_{1}, \alpha_{2}, \beta_{1}$ and $\beta_{2}{ }^{169}$.

Phenylephrine and clonidine are the agonists typically used to selectively activate $\alpha_{1-}$ and $\alpha_{2-}$ adrenoceptors, respectively. On the other hand, prazosin and yohimbinc antagonize the action of NA at these respective receptors. Langer was the first to propose that $\alpha_{1}$-adrenoceptors mediate the postsynaptic effects of NA and that $\alpha_{2}$-adrenoceptors serve autoreceptor functions regulating NA release ${ }^{258}$. Direct evidence that $\alpha_{2}$-adrenoceptors are localized on NA terminals was recently provided as the binding of $[3 \mathrm{H}]$ idazoxan, an $\alpha_{2}$-adrenergic ligand, was decreased in rat brain areas such as the cortex and the hippocampus after denervation of NA fibers ${ }^{200}$. However. most of [3H] idazoxan binding sites appear to be postsynaptic since around $80 \%$ of these remained unaffected by the noradrenergic

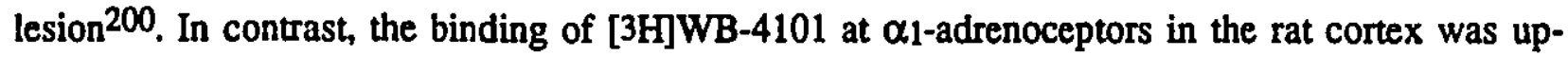
regulated following NA fiber denervation, suggesting that the latterreceptors are exclusively postsynaptic and become supersensitive following prolonged NA depletion 441 .

Advances in the techniques of pharmacology and molecular biology provided a more complex

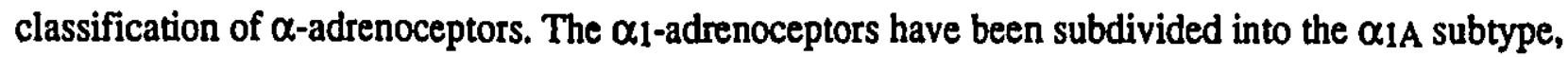
which has equal affinity for WB4101 and prazosin, and the $\alpha_{1 B}$ subtype that has a greater affinity for prazosin. The latter subtype is also more sensitive to inactivatior. by chlorethylclonidine ${ }^{195}$. Using the

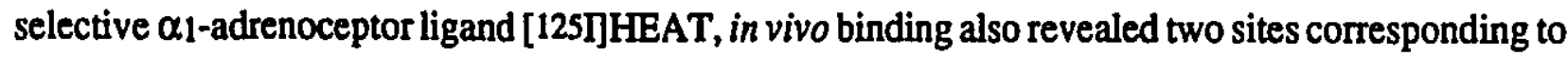

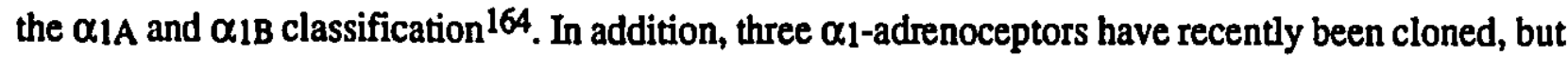
their characteris' $i$; hardly match those of the above pharmacological subtypes ${ }^{195}$. There is also ample evidence of heterogeneity at the level of the $\alpha_{2}$-adrenoceptors. The $\alpha_{2 A} \mathrm{~A}$ and $\alpha_{2 B}$ subtypes differ regarding their relative affinities for prazosin and oxymetazoline: the $\alpha 2 \mathrm{~A}$ subtype has a greater affinity for oxymetazoline while the $\alpha_{2 B}$ subtype has a higher affinity for prazosin $165,195,442$. There is, in addition, the $\alpha_{2}$ c subtype that resembles the $\alpha 2 \mathrm{~B}$ subtype but has a greater affinity ratio of prazosin versus oxymetazoline, and the $\alpha_{2 D}$ subtype that is comparable to the $\alpha_{2 A} \mathrm{~A}$ subtype but has a lower affinity for yohimbine as the main difference. Finally, there are four $\alpha_{2}$-adrenoceptors that have recently been cloned that correspond pharmacologically to the native $\alpha_{2 A}, \alpha_{2 B}, \alpha_{2 C}$ and $\alpha_{2 D}$ subtypes $195,277,442$. In 
situ hybridization studies in the rat brain revealed that the $\mathrm{LC}$ arsa is endo'wed with $\alpha 2 \mathrm{~A}$-adrenoceptors

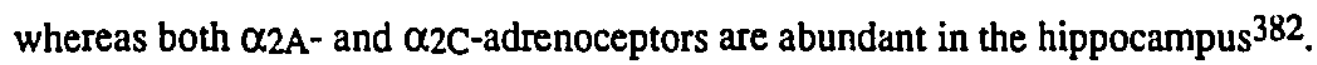

Many of the pharmacological effects that have been classically attributed to the activation of $\alpha$ adrenoceptors are in fact mediated by imidazoline ( $I_{1}$ and $I_{2}$ ) receptors that are insensitive to $\mathrm{NA}^{67,211,427,435}$. About $30 \%$ of [3H]para-aminoclonidine binding in the bovine brain cannot be dispiaced by NA and would correspond to I1 receptors ${ }^{133}$. Similarly, NA- resistant [3H]idazoxan binding sites would correspond to $I_{2}$ receptors ${ }^{211}$. Clonidine has a rather mediocre affinity for imidazoline sites compared to that for $\alpha_{2}$-adrenoceptors ${ }^{73}$. In turn, moxonidine and idazoxan are a good agonist and antagonist, respectively, at the imidazoline receptors, whereas yohimbine is inactive 134,211 . A putative neurotransmitter acting at imidazoline receptors has recently been discovered. Agmatine is an endogenous substance that binds potently to $I_{1}$ and $I_{2}$ receptors ${ }^{270}$. This substance is the product of the enzymatic decarboxylation of arginine, an important component of the urea cycle, present in the brain 305 .

There are also the two $\beta_{1}$ and $\beta_{2}$-adrenoceptors. However, the amino acid sequence disparity between these subtypes is not as great as for $\alpha_{1}$ - and $\alpha_{2}$-adrenoceptors ${ }^{461}$. In the brain, $\beta_{1}$ and $\beta_{2}$ adrenergic receptors can hardly be differentiated in terms of their physiological functions since they both stimulate adenylate cyclase ${ }^{461}$. Nevertheless, autoradiographic studies revealed that these receptors are differentially regulated by NA fiberdenervation, and have distinctdistributions in the brain 309 . )-[125I]Iodocyanopindol in the presence of unlabeled 5-HT (to mask 5-HT1B receptors) binds with a single high affinity site to $\beta_{1}$ - and $\beta_{2}$-adrenoceptors in the hippocampus. Competition analysis with drugs selective for $\beta$-adrenoceptor sivitypes (CI-89406 and CI-118551) revealed that about $70 \%$ of $\beta$ adrenoceptors in the hippocampus are of the $\beta_{1}$ subtype, the remainder being of the $\beta_{2}$ subtype ${ }^{436}$.

\section{Physiology of serotonergic and noradrenergic neurons}

\subsection{Serotonergic neurons}

The serotonergic innervation of the hippocampus arises from 5-HT neurons of the median and dorsal raphe area (DR and MR) ${ }^{98}$. As originally describcd by Aghajanian and co-workers, most neurons of this region display a slow and regular firing rate and long-duration, positive action potential. This firing pattern was shown as being characteristic of 5-HT-containing neurons using different techniques: combined single cell recording and fluorescence histochemistry, stimulation of the ascending 5-HT pathway coupled with antidromic recording, and 5-HT denervation 4,457 . The firing activity of 5-HT neurons is attributed to a pacemaker cycle involving a calcium-dependent potassium current. After an action potential, 5-HT neurons exhibit a large afterhyperpolarization (AHP) that decays slowly during the interspike interval. Spikes are generated by the termination of the AHP rather than by excitatory postsynaptic potentials (EPSPs) arising from afferent fibers. These events can be explained in molecular terms: during depolarization, the extracellular calcium enters the cell through voltage-dependent calcium channels and then activates a transient outward potassium conductance which consequently 
leads to an AHP. Calcium is then extruded and sequestered to allow the decay of the AHP and the deinactivation of the calcium current. When the membrane voltage finally reaches the threshold for the activation of voltage-dependent calcium conductances, another action potential is triggered ${ }^{6}$.

The firing rate of 5-HT neurons is regulated by autoreceptors of the 5-HT1A subtype localized in the somatodendritic region. Indeed, microiontophoretic application of 5-HT or 5-HT1A agonists (e.g. 5-methoxytryptamine, 8-OH-DPAT, LSD, ipsapirone, gepirone) onto dorsal raphe 5-HT neurons depresses their firing activity, and this suppressant effect is antagonized by intravenous spiperone or by microiontophoretically applied (-)-propranolo151,61,64,275,368,412. The reduction of 5-HT neurons' discharges induced by $5-\mathrm{HT}_{1 \mathrm{~A}}$ receptor activation is mediated by an hyperpolarization of the membrane occurring through an opening of potassium channels $5,413,466$. Pertusis toxin inactivation of $\mathrm{G}_{\mathrm{i} / \text { oproteins }}$ markedly reduces the suppressant effect of 5-HT1A agonists 60,228 . However, in vitro application or intracellular injection of cAMP analogues do not reduce this response ${ }^{228}$. It thus appears that 5-H $\mathrm{T}$ autoreceptors activate $G$ proteins which can directly open potassium channels without any involvement of adenylate cyclase.

Microdialysis and voltammetry studies have shown that the activation of 5-HT1A autoreceptors in the raphe area decreases the release of 5-HT at the level of terminals in the hippocampus ${ }^{63,401}$. It thus appears that changes in the firing rate of 5-HT neurons induced by drugs acting at 5-HT1A autoreceptors can alter the level of activation of postsynaptic 5-HT receptors in the brain. However, it is still not clear to what extent physiological variations in raphe cell firing can alter the release of 5-HT at postsynaptic sites. In any case, the firing activity of 5-HT neurons appears quite stable during the aroused period and the only major changes are those associated with the sleep-wake cycle 233 . During REM sleep 5-HT neurons are silent, whereas during awakening these neurons discharge at rates of about $3 \mathrm{~Hz}$ during quiet periods and of about $6 \mathrm{~Hz}$ during arousal ${ }^{440}$. It is noteworthy, however, that various environmental stimuli or stressors do not change the discharge rate of 5-HT neurons when compared to the undisturbed active waking baseline 465 .

\subsection{Noradrenegic neurons}

The hippocampus is innervated by NA terminals originating exclusively from the locus coeruleus (LC) 191,241. Similarly to 5-HT neurons in raphe nuclei, NA neurons in the LC have been shown to display a slow, tonic pacemaker activity with long-duration action potentials 177 . This spontaneous activity appears intrinsic to NA neurons because it is still present in dissociated LC NA neurons in culture ${ }^{292}$. A scheme of a pacemaker cycle of action potentials involving calcium-activated potassium conductances, similar to the one depicted above for 5-HT neurons, can explain some aspects of NA neurons' physiology. However, this pacemaker activity also shows a dependence on cAMP and protein kinase A. Extracellular agents that increase CAMP levels enhanced the firing rate of $L C$ neurons ${ }^{459}$. In addition, LC NA neurons in vitro show a time-dependent decrease of their spontaneous firing activity that can be counteracted with intracellular infusion of CAMP, 8-Br-cAMP, or with the catalytic subunit of cAMP-dependent protein kinase, and this effect is accentuated with a specific inhibitor of this enzyme ${ }^{15}$. 
The firing activity of LC NA neurons is regulated by somatodendritic autoreceptors of the $\alpha_{2}$ adrenergic subtype. Indeed, intravenous administration or microiontophoretic application of clonidine inhibits the firing activity of these NA neurons, and this effect is blocked by $\alpha_{2}$-adrenergic antagonists such as yohimbine $83,291,431$. Elevated synaptic levels of endogenous NA, achieved through the blockade of NA reuptake carriers with desipramine, or microiontophoretic application of NA also produce an inhibition of firing of LC NA neurons that is blocked by the $\alpha_{2}$-adrenergic antagonist idazoxan 105 . Activation of the somatodendritic NA autoleceptors by endogenous NA is further demonstrated by the fact that small intravenous doses of $\alpha_{2}$-adrenergic antagonists increase, by themselves, the discharge rate of NA neurons 291 .

There is little doubt that $\alpha_{2}$-adrenergic receptors in the LC decrease the firing rate of NA neurons primarily via an increase in potassium conductance. Agonists at $\alpha_{2}$-adrenergic receptors produce a hyperpolarization of these neurons whose reversal potential is predicted by the Nernst equation for a potassium-dependent change in permeability 19,467. At high concentrations, NA can also decrease calcium currents. However this effect is not mimicked by clonidine nor prevented by yohimbine ${ }^{468}$, thus excluding a primary involvement of calcium conductance in the mediation of $\alpha_{2}$-adrenergic autoinhibition at this level. Somatodendritic $\alpha_{2}$-adrenergic autoreceptors might increase the potassium conductance through an inhibition of adenylate cyclase. Inactivation of $\mathrm{Gi} / 0$ proteins with pertussis toxin was shown to almost totally abolish the effect of clonidine at these receptors 7 . Furthermore, intracellular injection of cAMP analogues reversed $\alpha_{2}$-adrenoceptor-mediated hyperpolarization ${ }^{19}$. Elevation of cAMP concentrations increased the firing rate and produced an inward current in LCNA neurons ${ }^{8}$. However, this inward current was not simply the opposite of the outward potassium current elicited by $\alpha_{2}-$ adrenergic agonists since it was not reversed in the region of potassium reversal potential ${ }^{8}$. According to Aghajanian and Wang ${ }^{8}, \alpha_{2}$-adrenergic agonists would have a dual action. Through a direct action of the $\mathrm{G}$ protein transduction system, they would open potassium channels without involving cAMP. Secondly, $\alpha_{2}$-adrenergic agonists would induce a $G$ protein-mediated inhibition of adenylate cyclase, that, in turn, inactivates a cAMP-dependent inward current. These two phenomena would act in concert to produce the $\alpha_{2}$-adrenergic-mediated hyperpolarization of LC NA neurons.

The discharge rate of LCNA neurons during the sleep-wake cycle is comparable to that discussed above for 5-HT neurons of raphe nuclei (i.e., being highest during waking states, lowest during deep sleep and virtually silent during REM sleep) ${ }^{24}$. Contrary to 5-HT neurons, LC NA neurons increase markedly their firing activity in response to noxious, stressful, or anxiogenic stimuli231,361,362. At the postsynaptic level, a stress stimulus and the direct electrical stimulation of the LC area reduce the activity of hippocampal neurons ${ }^{398}$ and increase the extracellularconcentration of NA in the hippocampus $2,245,330$.

It is difficult to determine with classical pharmacological tools how changes in the firing rate of LC neurons can affect the release of NA in the hippocampus since both somatodendritic and terminal autoreceptors are of the $\alpha_{2}$-adrenergic subtype. Nevertheless, the release of NA per pulse in the hippocampus was shown to be inversely related to the frequency of the stimulation of the NA pathway 
arising from the $\mathrm{LC}^{104}$. This result suggests that in the situation of high neuronal discharge, terminal autoreceptors decrease the amount of NA released per action potential. Thereby, in vivo administration of $\alpha_{2}$-adrenergic agonists could decrease the release of NA in the hippocampus by acting mainly at terminals, rather than at somatodendritic autoreceptors. Here is the evidence supporting this contention. First, intraperitoneal injection of clonidine or idazoxan modulates NA turnover in brain terminal areas equally well in rats that had $\mathrm{LC}$ inactivation with tetrodotoxin as in intact rats ${ }^{106}$. Second, low doses of the alkylating agent EEDQ (N-ethoxycarbonyl-2-ethoxy-1,2-dihydroquinoline) antagonize the effect of clonidine on the terminal NA turnover, while not affecting the inhibitory effect of this drug on the firing rate of the LC NA neurons ${ }^{130}$.

\section{Distribution of 5-HT and NA innervations of the hippocampus}

The 5-HT and NA systems are known to sourse along three common bundles of fibers to reach the hippocampus: the fimbria-fornix, the fasciculus cinguli, and the amygdaloid pathway. All NA fibers of these bundles arise from the LC area ${ }^{191,241}$. In contrast, 5-HT fibers of the hippocampus originate from distinct raphe nuclei. Neurons of the MR project to the hippocampus via the fasciculus cinguli and the fimbria-fornix, whereas those of the DR project to the hippocampus via the amygdaloid pathwoy 26,437. MR and DR 5-HT fibers exhibit readily recognizable morphological differences. Serotonergic axons of the MR are relatively coarse with large spherical varicosities (enlargements of aggregated synaptic vesicles containing 5-HT). In contrast, axons from the DR are very fine and have small varicosities of various forms 232,284 .

The fimbria-fornix contributes to the NA arborization of $\mathrm{CA} 3$ stratum lucidum (at the apical side of the pyramidal cell layer) and to the polymorph layer of the dentate gyrus (fig. 3)191,335. On the other hand, 5-HT fibers carried by the latter bundle arborize to the stratum lacunosum-moleculare of $\mathrm{CA}_{1}$ and the stratum radiatum of $\mathrm{CA}^{26}$ as well as to the molecular and the polymorph layer of the dentate gyrus. The fasciculus cinguli distributes its NA and 5-HT fibers to the molecular layers of the dentate gyrus, the subiculum and the Ammon's horn 191,314. The amygdaloid bundle distributes a dense NA innervation in the whole hippocampal formation, but sends 5-HT fibers mainly to the molecular layer of the dentate gyrus 26,80 .

Physiological interactions between the 5-HT and NA systems require a relative proximity of the terminals that release these neurotransmitters. It is of interest here to compare the relative distribution of these fibers in various layers of the hippocampal formation. Oleskevich and co-workers have recently quantified the distribution of NA and 5-HT fibers in the rat hippocampus using [3H]NA and [3H]5-HT uptake-labeling of axonal varicosities 334,335 . They found these varicosities to be prominent in all regions of the hippocampus, suggesting multiple sites for interactions between the 5-HT and NA systems (Fig.3). A dense labeling of both [3H]NA and [3H] 5-HT is found in the apical layers (molecular and radiatum) of all regions (subiculum, Ammon's horn and dentate gyrus), except for the stratum lucidum of Ammon's hom that receives a large amount of NA but not of 5-HT varicosities. In addition, these neurotransmitter systems largely innervate the polymorph layer of the subiculum, whereas the stratum 
Serotonergic innervation of the hippocampus

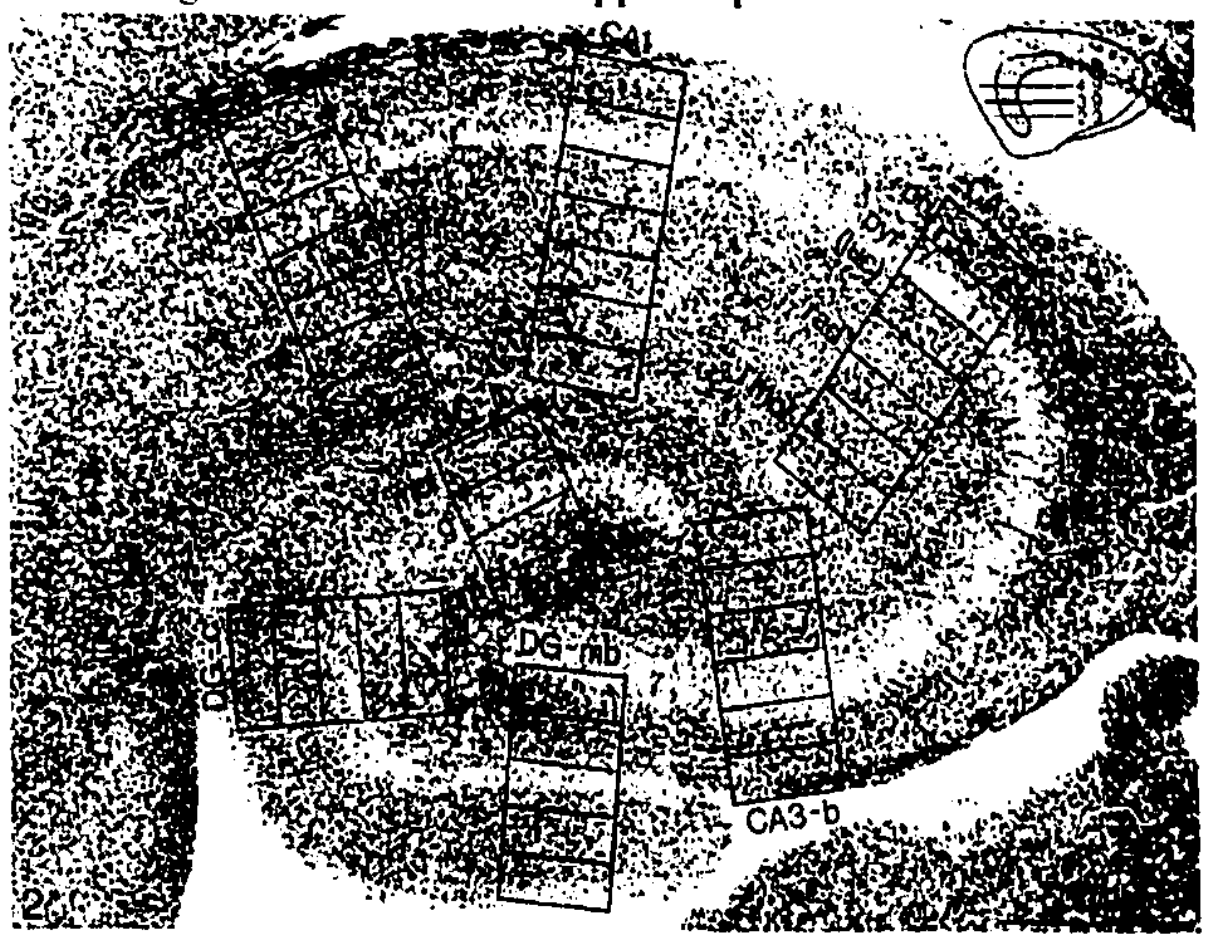

Noradrenergic innervation of the hippocampus

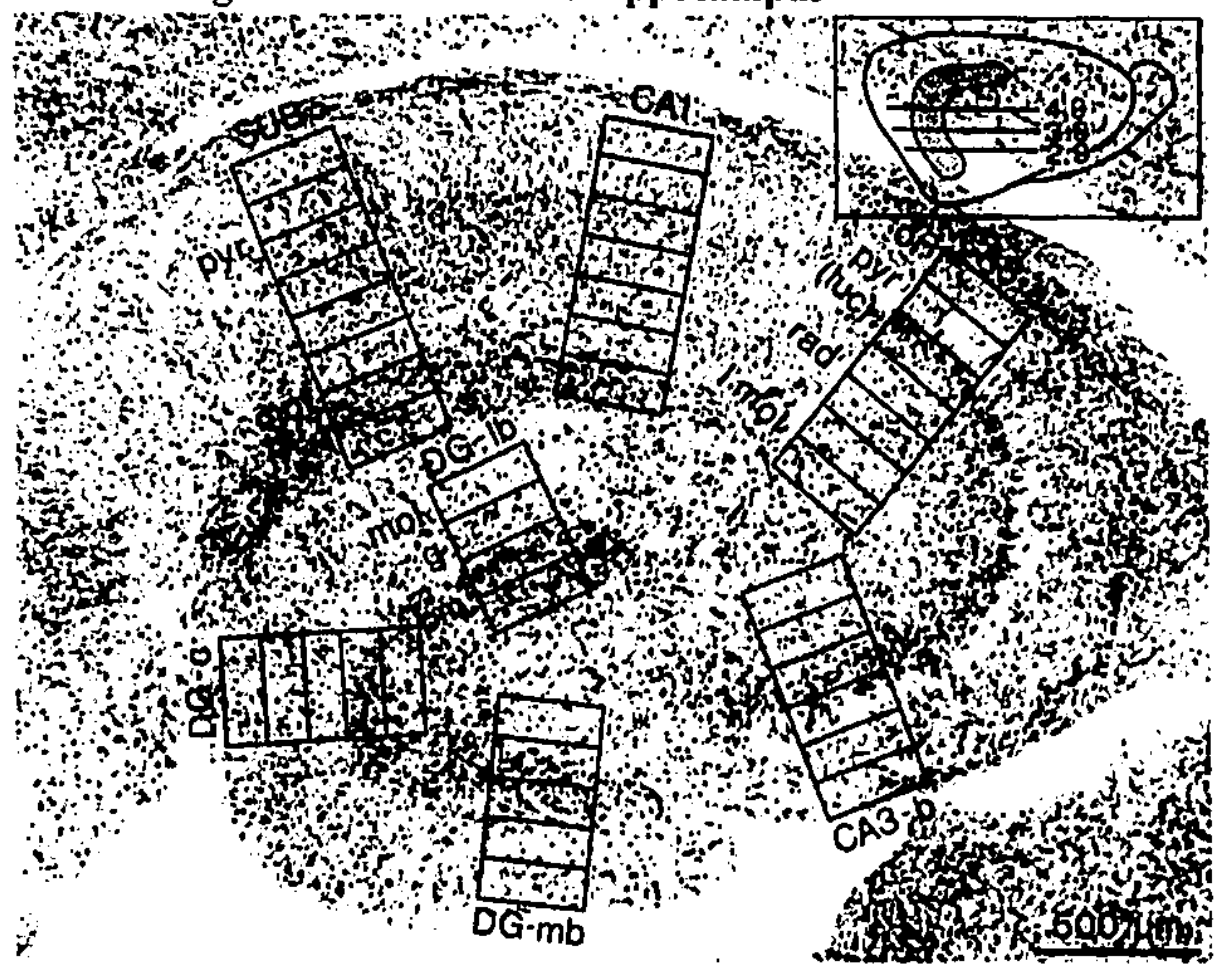

Fic 3. NA and S-HT innervations of tho hippoeampus. SUB, subienlam; CA1, CA3-a and CA3-b, respective Ammon's horn sectors; DG-mb, DGe and DG-1b, medial blade, ereat and lateral blade of the deatnte gyress. The various Iayers in each repion are designated alongaide their respective countiog windows by the following abbreviations: pyr, stratum pyramilale; mol, stratum moloculare; or, stratum orieas; luc, stratum rucidum; rad, stratum radiatum; Imol, stratum pyramidale; n10h, stratum molevelare; of, stratum oriens; les, stratum lucidum; rad, stratum radiatum; Insol, stratum lacunosum-moiecuiare; E, granalo cell byer; pm, polymorph cell layer (courteay of L. Descarries). 
oriens is an area enriched with 5-HT varicosities. In contrast, there is only sparse NA and 5-HT innervation in the pyramidal and granular cell layers and in the alveus of Ammon's horn.

The relative density of varicusities containing NA compared with those containing 5-HT in different layers of the hippocampal formation was estimated from the work of Oleskevich and coworkers 334,335 (fig. 3). At the : $:$ vel of the apical projections of pyramidal and granular cells (stratum moleculare and radiatum), the ratio of 5-HT/NA varicosities is almost two. This ratio is even greater (almost four) at the level of the stratum oriens of Ammon's horn. In contrast, there are slightly more NA varicosities in the region of the pyramidal cell layers of $\mathrm{CA}_{3}$, as well as in the granular and polymorph layers of the dentate gyrus. Finally, the ratio of 5-HT/NA terminals in the pyramidal layers of the CA1 and of the subiculum is similar to that found at the level of the molecular layers.

\section{Serotonergic neurotransmission in the hippocampus}

\subsection{Presynaptic mechanisms}

Contrary to somatodendritic autoreceptors of the raphe area, terminal 5-HT autoreceptors of the hippocampus are not of the 5-HT1A subtype, and control the release of 5-HT without interfering with the propagation of action potentials ${ }^{423}$. In vitro characterization using rat hippocampal slices preloaded with [3H]5-HT, reveaied that these autoreceptors are of the 5-HT1B subtype ${ }^{140}$. For instance, 5-CT potently decreases the evoked release of $[3 \mathrm{H}] 5-\mathrm{HT}$, and this effect is blocked by methiothepin, metergoline and cyanopindolol. In contrast, 8-OH-DPAT is much less potent at inhibiting the release of 5-HT, and this inhibition is not blocked by spiperone ${ }^{140}$. Methiothepin increases by itself the evoked release of [3H]5-HT from preloaded hippocampal slices $140,218,343,456$, whereas metergoline does it only in the presence of a 5 -HT reuptake blocker ${ }^{140}$, and $\beta$-adrenergic antagonists such as cyanopindolol and propranolol act as partial agonists at 5-HT1B autoreceptors 140,296 . The role of these hippocampal autoreceptors on 5-HT terminals has also been investigated in vivo. Electrical stimulation of the 5-HT pathway suppresses the firing activity of pyramidal neurons by releasing synaptic 5-HT48,49. The effectiveness of the stimulation is decreased by the administration of RU 24969, a 5-HT1A/1B agonist, and this effect is blocked by methiothepin, but not by a 5-HT1A antagonist, BMY 737886,88. Furthermore, a microdialysis study revealed that local application of the specific 5-HT1B agonist CP93,129 decreases the hippocampal output of endogenous 5-HT and that this effect is also blocked by methiothepin 213 .

Terminal 5-HT autoreceptors in species other than rodents are of the 5-HTID subtype. Human and guinea-pig hippocampi are endowed with 5-HT1D rather than 5-HT 1 B receptors ${ }^{222}$, but there are no data indicating that terminal autoreceptors in the hippocampus of these later species are also of the 5-HTID subtype. Nonetheless, in vitro superfusion experiments have been carried out using human or guinea-pig neocortical slices 158,307 . In both cases, 5-CT decreased the release of [3H]5-HT. These inhibiting effects were blocked by the 5-HT1B/1D antagonist methiothepin, but not by the 5-HT1B antagonist cyanopindolol 158,307. 
Most authors agree that terminal 5-HT autoreceptors decrease the release of 5-HT by reducing the calcium influx via voltage-dependent calcium channels ${ }^{423}$. Three series of findings support this contention: 1) The effectiveness of the activation of terminal 5-HT autoreceptors is less pronounced the higher the calcium concentration in the superfusion medium ${ }^{175}$;2) The calcium-independent release of $[3 \mathrm{H}] 5-\mathrm{HT}$ by fenfluramine is not modulated by ligands acting at terminal 5 -HT autoreceptors ${ }^{259} ; 3$ ) Voltage-sensitive calcium channel agonists (dihydropyridines) concentration-dependently enhance potassiumevoked release of $[3 \mathrm{H}] 5-\mathrm{HT}^{141}$. It is not clear, however, what are the molecular steps involved in the transduction of the autoinhibitory 5-HT signal that lead to a change in calcium influx into 5-HT terminals. Although the activation of protein kinase $C$ was found to enhance the release of $[3 \mathrm{H}] 5-\mathrm{HT}$ from hippocampal slices, it did not alter the effect of 5-HT agonists and antagonists acting at terminal autoreceptors ${ }^{138}$. There is one report indicating that the inhibitory effect of LSD on the release of 5-HT is abolished in slices preincubated 4 hours with the $\mathrm{G}_{\mathrm{i} / \mathrm{o}}$ proteins ribosylating agent pertussis toxin, suggesting that terminal 5-HT autoreceptors are linked with these $G$ proteins ${ }^{343}$. However, the lack of effect of this pretreatment on the facilitatory effect of methiothepin ${ }^{343}$ does not fit with this hypothesis. Furthermore, subsequent studies revealed no changes in the function of terminal 5-HT autoreceptors following in vivo inactivation of either $\mathrm{G}_{\mathrm{i} / \mathrm{o}}$ or $\mathrm{G}_{\mathrm{s}}$ with pertussis or cholera toxin, respectively, and following in vitro inactivation of $G$ proteins with $\mathrm{N}$-ethylmaleimide ${ }^{41,60}$.

Evidence was recently provided for 5-HT3 receptors having an opposite role on the release of 5HT compared to the 5-HT1B/1D autoreceptors. Indeed, these receptors would exert a positive feedback on the release of 5-HT43,290. The selective 5-HT3 agonist 2-methyl-5-HT and elevated levels of endogenous 5-HT (achieved through the blockade of the 5-HT reuptake carrier) increase the evoked release of [3H]5-HT from preloaded guinea pig hippocampal slices, effects that are blocked by various 5-HT3 antagonists ${ }^{43}$. A microdialysis study revealed similar phenomena in the rat hippocampus 290 . These 5-HT3 receptors do not appear to be tonically antivated since the 5-HT3 antagonist MDL 7222 does not decrease by itself the release of [3H]5-HT in vitro, nor that of endogenous 5-HT in vivo ${ }^{43,290}$. Much research is needed to determine the mechanisms involved in the facilitatory action of 5-HT3 receptors on the release of [3H]5-HT since the cellular localization of these receptors is not even known. A direct action of 5-HT3 agonists at receptors on 5-HT terminals was ruled out by the finding that these ligands fail to enhance the release of $[3 \mathrm{H}] 5-\mathrm{HT}$ in experiments using nerve endings (synaptosomes) or using slices that had nerve influx propagation blocked by the inactivation of the sodium channels with tetrodotoxin 62 .

\subsection{Postsynaptic mechanisms}

\subsubsection{Single-unit studies}

Early in vivo electrophysiological studies revealed that microiontophoretic upplication of 5-HT onto neurons of the hippocampus produces predominantly inhibitory effects, but also sometimes excitatory effects. At the time of these early investigations, only the excitatory effect was known to be 
blocked by classical 5-HT antagonists such as methysergide 187,366 . More recent in vivo studies have shown that the inhibitory effect of 5-HT on the firing rate of pyramidal neurons of hippocampus is blocked by the 5-HT1A antagonist BMY 7378, but not by the 5-HT2 antagonist ketanserin 61,87 . Furthermore, selective ligands of 5-HT 1 A receptors such as 8-OH-DPAT, gepirone or tandospirone act as partial agonists at these receptors. When applied by microiontophoresis, these ligands display suppressanteffects on the firing rate of pyramidal neurons, but also antagonize the effect of 5-HT applied concomitantly53,61,87,166. There are apparently two subsets of postsynaptic 5-HT1A receptors in the hippocampus differentially coupled to $G$ proteins that suppress pyramidal cell firing. Indeed, the extrasynaptic 5-HT1A receptors localized on the soma of pyramidal cells, which can be activated by microiontophoretic application of agonists, are inactivated by pertussis toxin 60 . On the other hand, intrasynaptic 5-HT $1 \mathrm{~A}$ receptors localized on dendrites of pyramidal cells and activated by endogenous 5-HT released by the electrical stimulation of the ascending 5-HT pathway are unaffected by pertussis toxin ${ }^{60}$. It thus appears that extrasynaptic $5-\mathrm{HT}_{1 \mathrm{~A}}$ receptors are coupled with $\mathrm{G}_{\mathrm{i} / 0}$ proteins while intrasynaptic 5-HT $1 \mathrm{~A}$ receptors are not.

In vitro intracellular recordings have revealed that the most prominent effect of 5-HT on hippocampal pyramidal neurons is a membrane hyperpolarization associated with an increase in potassium conductance $97,234,395$. This response most likely constitutes the membrane mechanism underlying the suppressant effect of 5-HT on the firing rate of pyramidal neurons. First, the opening of this potassium channel by 5-HT was shown to be mediated by 5-HT 1 A receptors as it is blocked, for instance, by 8-OH-DPAT and spiperone and mimicked with a lesser efficacy by 8-OH-DPAT $21,96,369$. Second, the ability of 5-HT1A receptors to hyperpolarize pyramidal neurons is prevented by pertussis toxin. Finally, intracellular injection of GTP $\gamma$ S, a non-hydrolyzable analogue of GTP, produces a persistent hyperpolarization and prevents the effect of 5-HT, showing an involvement of $\mathrm{G}_{\mathrm{i} / \mathrm{o}}$ proteins in this electrophysiological response ${ }^{18,481}$.

Superfusion of 5-HT was also shown to inhibit cAMP formation from hippocampal membranes in which adenylate cyclase was activated by forskolin in the presence or absence of vasoactive intestinal peptide ${ }^{113,122}$. The 5-HT1A ligand 8-OH-DPAT is a full agonist in this paradigm, whereas spiperone is an antagonist ${ }^{113,122}$. Interestingly, the activation of 5-HT1A receptors was also shown to stimulate the production CAMP in hippocampal membranes 402 , a result consistent with the electrophysiological data discussed above, indicating that there might be two subsets of 5-HT 1 A receptors in this brain area coupled to either $G_{i}$ or $G_{s}$ proteins. It is important to emphasize, however, that the $G$ protein-coupled 5-HT1A receptors that mediate the hyperpolarization of pyramidal neurons activate potassium channels without involving the stimulation or the inhibition of adenylate cyclase. Indeed, intracellular injections of forskolin or 8-Br-cAMP did not hyperpolarize pyramidal neurons. Moreover, buffering intracellular CAMP with 8-Br-cAMP failed to blunt the hyperpolarization effect of 5-HT 18 . Th., exact functions of these 5-HTIA-mediated modulations of adenylate cyclase in 5-HT neurotransmission remain unknown.

In addition to its hyperpolarizating action, 5-HT elicits on hippocampal neurons late excitations that are not mediated by $5-\mathrm{HT}_{1 \mathrm{~A}}$ receptors ${ }^{21,97}$. Suppression of two types of potassium conductances 
would be involved in these effects of 5-HT: first, a suppression of a slowly developing calciumdependent potassium current (I $\mathrm{I}_{\mathrm{H} H \mathrm{P}}$ ) would be responsible for the accommodation of cell firing frequency (the length of the AHP is reduced by 5-HT via the suppression nf this current and c. nnsequently leads to increased neuronal discharges in response to depolarizaliun inputs), and, second, an even more slowly developing and long-lasting suppression of a voltage-dependent potassium currents ( $\mathrm{Im}$ ) that produces neuronal depolarizations and excitations 21,97 . In pyramidal neurons of guinea pig and rat cortex, for instance, these responses have been attributed to the activation of 5-HT2 receptors ${ }^{167}$. However, Andrade and Nicoll 21 have shown that 5-HT-induced suppressions of either $I_{m}$ or IAHP in hippocampal pyramidal neurons are not blocked by ketanserin, thus excluding any involvement of 5 HT2 receptors in this brain area. Subsequent studies suggested that the suppression of potassium conductances by 5-HT are mediated by 5-HT4 receptors since they are blocked by the 5-HT3/4 agents BRL 24924, zacopride and cisapride, and mimicked by the 5-HT $1 / 4$ agonists 5-methoxytryptamine and 5-CT, but not by the 5-HT3 agonist 2-methyl-5-HT 20,85. Interestingly, 5-HT4 receptors have been found to be positively coupled to adenylate cyclase system in the guinea pig hippocampus $120,121,402$. However, further studies are awaited to establish whether the above electrophysiological effects are mediated through this second messenger system.

In the presence of spiperone (which blocks the 5-HT1A and 5-HT2A receptors), a fast excitatory response of pyramidal cells to 5-HT was also observed, in addition to the late excitatory response described above. The former response to 5-HT was mimicked by the 5-HT2A/C receptor agonist DOI, and antagonized by the 5-HT2A/2Cligand ketanserin, suggesting the involvement of 5-HT2Creceptors 36 . It was concluded that 5-HT can increase the subthreshold excitatory postsynaptic potential (EPSP) amplitude to initiate spike firing via these receptors. However, the exact nature of the ion channels involved is unknown 36 .

Serotonin mediates depolarization in a variety of tissues by the opening of 5-HT3 receptorchannels that have mixed permeability to sodium and potassium 350,474 . In cultured embryonic mouse hippocampal cells, 5-HT and 2-methyl-5-HT induce large inward currents that are short lasting and of rapid onset, and these responses are blocked by the 5-HT3 antagonist tropisetron ${ }^{475}$. A major characteristic of the 5-HT3 receptor-channels is that they rapidly desensitize following prolonged 5-HT application. Moreover, the function of these 5-HT3 receptors appears to be regulated by the adenylate cyclase system since the rate of desensitization was found accelerated by the perfusion of forskolin, phosphodiesterase inhibitors, cAMP analogues or cholera toxin 475 .

It is important to note that 5-HT3-receptor-mediated membrane depolarizations were observed on a heterogeneous population of hippocampal cells 475 , but not at the level of hippocampus pyramidal cells 21,97 . Recent evidence suggests that the activation of 5-HT3 receptors excites GABAergic interneurons which would in turn inhibit the firing activity of pyramidal neurons through GABAA receptors. Anatomical studies revealed synaptic contacts between 5-HT terminals and GABAergic interneurons of the hippocampus 153 . Furthermore, 5-HT and 2-methyl-5-HT increase inhibitory synaptic inputs and these effects are blocked by tropisetron as well as by the GABAA antagonist bicuculline 370 . 


\subsubsection{Multi-unit studies}

Serotonergic neurotransmission was also investigated electrophysiologically in the hippocampus with the technique of field potential recording, which allows the determination of the firing activity of a population of neurons (population spike). The effect of 5-HT on field potential is a composite of two responses mediated by at least two distinct 5-HT receptors. Bath application of 5-HT produces an initial increase followed by a decrease in the amplitude of the $\mathrm{CA}_{1}$ population spike evoked by the stimulation of the stratum radiatum 38,372 . The suppressing effect of 5-HT appears to be of the 5-HT 1 A subtype as it was mimicked by 8-OH-DPAT and blocked by spiperone, but not by ketanserin ${ }^{37}$. Furthermore, the CA1 population spike evoked by the stimulation of the Schaffer collaterals was also reduced by 8-OHDPAT and buspirone ${ }^{348}$.

This inhibitory effect of 5-HTIA agonists might be explained by the hyperpolarization of pyramidal neurons that we have reviewed in the preceding section. Consistently, the decrease in population spike amplitude induced by $5-\mathrm{HT}$ was also blocked by the inactivation of $\mathrm{G}_{\mathrm{i}} / \mathrm{o}$ proteins with pertussis toxin 94 . The excitatory effect of 5-HT, however, cannot be explained by the 5-HT4-receptormediated decrease in the potassium conductance, characterized by Andrade and Chaput ${ }^{20}$, since in contrast to the 5-HT blockade of afterhyperpolarization, the 5-HT-induced enhancement in population spike amplitude has a rapid onset ${ }^{38,372}$. This excitatory response of $5-\mathrm{HT}$ would best be explained by the 5-HT2C receptors that increase a subthreshold cationic conductance ${ }^{36}$ (see the preceding section).

Interestingly, 5-HT was also shown to inhibit the induction of prolonged enhancements in the excitability of hippocampus pyramidal neurons. High frequency stimulation of mostexcitatory synapses of the hippocampus lead to long-term potentiation (LTP) of synaptic transmission. Subsequent stimulations of these excitatory pathways typically produce population spikes of greater amplitude, a phenomenon thought to be of relevance to memory processes ${ }^{126}$. Many studies have indicated that high frequency stimulation of excitatory pathways depolarizes the membrane of postsynaptic neurons through the activation of glutamate receptors (kainate/quisqualate), which then allows the activation of voltage-sensitive glutamate receptors, the NMDA receptor-channels. These channels permit influx of calcium into the cell, that, in turn, triggers second messenger events, such as the activation of protein kinase, thought to be crucial for the production of LTP76. Production of LTP by high frequency stimulation of either the commisural pathway projecting to the $\mathrm{CA} 3$ region or of the stratum radiatum in the $\mathrm{CA}_{1}$ region were shown to be blocked by bath superfusion of $5-\mathrm{HT}^{100,454}$. The blockade of LTP by 5 -HT could involve a hyperpolarization of pyramidal neurons through $5-\mathrm{HT}_{1} \mathrm{~A}$ receptors as this effect is blocked by methysergide or by spiperone and mimicked by $5-\mathrm{CT}^{100,454}$. The suppressant effect of 5-HT on LTP in the CA 1 region seems to involve also 5-HT3 receptors because this response is blocked by tropisetron and ondansetron ${ }^{100}$. It was suggested that 5 -HT3 receptors that reside on the GABAergic interneurons and mediate inhibition of the firing activity of pyramidal neurons ${ }^{370}$ could be implicated in the suppression of LTP by $5-\mathrm{HT}^{100}$. 


\section{Noradrenergic neurotransmission in the hippocampus}

\subsection{Presynaptic mechanisms}

Noradrenergic fibers of the hippocampus are endowed with $\alpha_{2}$-adrenergic autoreceptors that control the amount of NA released from terminals, but not the propagation of action potentials or the synthesis of NA as do their somatodendritic counterparts ${ }^{422}$. Ilustrating the inhibitory effect of presynaptic $\alpha 2$-adrenoceptors, nanomolar concentrations of NA or cloniairie decrease the electricallyor potassium-evoked release of $[3 \mathrm{H}] \mathrm{NA}$ from preloaded slices of rat and rabbit hippocampus, and these effects are blocked by yohimbine. In contrast, the $\alpha_{1}$-adrenergic agonist phenylephrine produces a similar inhibiting effect only at micromolar concentrations, and the latter effect is not blocked by the

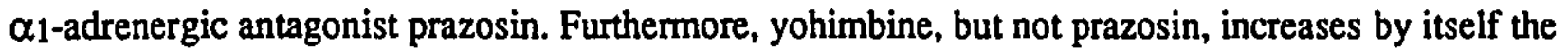
evoked release of [3H]NA in the hippocampus ${ }^{149,230}$. However, $\alpha 2$-adrenergic antagonists are without any effect in conditions where there is no autoinhibition of NA release ${ }^{11}$. In vivo electrical stimulation of the ascending NA pathway of the locus coeruleus has also been shown to suppress the firing activity of hippocampal pyramidal neurons ${ }^{104}$. The effectiveness of the stimulation is decreased by clonidine, increased by idazoxan, and inversely proportional to the frequency of stimulation, confirming that the receptors mediating these effects are terminal $\alpha_{2}$-adrenergic autoreceptors ${ }^{104}$. Recently, more precise pharmacologicai characterization revealed that terminal autoreceptors are of the $\alpha_{2 \mathrm{D}} \mathrm{subtype}$ in the rat and of the $\alpha_{2 A}$ subtype in the rabbit ${ }^{438}$. Additional characterization of receptors on NA terminals of hippocampus will be required to determine whether imidazoline receptors are implicated in the inhibition of the release of NA by agents such as clonidine and oxymetazoline, as was found to be the case at the level of postganglionic sympathetic nerve endings 173 .

There are still controversies about the exact molecular mechanisms involved in the inhibition of NA release by presynaptic $\alpha_{2}$-adrenoceptors in the central nervous system. Pertussis toxin and $\mathrm{N}$ -

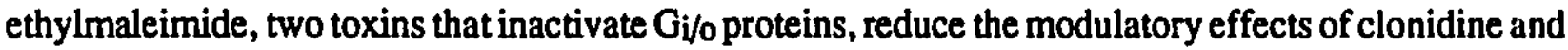
yohimbine on the release of [3H]NA in rabbit hippocampal slices ${ }^{12,13}$. Although the issue has not been investigated yet in hippocampus, an analog of cAMP (8-bromo-cAMP) and activators of adenylate cyclase ( $\mathrm{NaF}$ or forskolin) were shown to increase the release of [3H]NA and reduce the modulating effect of $\alpha 2$-adrenergic agonists and antagonists in rat hypothalamus and cerebral cortex 359,389 . In contrast, activation of protein kinase $\mathrm{C}$ with phorbol esters increases the release of [3H]NA, but does not change the effect of yohimbine ${ }^{208}$. These results suggest that terminal autoreceptors reduce the release of NA through the $G_{i}$ protein-mediated inhibition of adenylate cyclase. This interpretation has to be taken with caution, however, considering that divergent results were reported with forskolin 289 and that some authors estimate the magnitude of the effect of pertussis toxin on terminal autoreceptor function as being weak compared to what is observed at the level of postsynaptic $\alpha 2$-adrenoceptors ${ }^{329}$.

The primary ion conductance affected by terminal $\alpha 2$-adrenergic autoreceptors is also a matter of debate. As has been observed for somatodendritic $\alpha 2$-adrenergic autoreceptors, terminal autoreceptors could increase a potassium conductance, hyperpolarize the axons, and thereby decrease the influx of calcium through voltage-dependent calcium channels. In support of this view, the inhibitory effect of 
this $\alpha_{2}$-adrenergic-mediated hyperpolarization is prevented when a very high concentration of potassium is used to evoke [3H]NA release in rathippocampal synaptomes 69,422 . Furthermore, the effect of clonidine in rat hippocampal slices is distinctly diminished in the presence of a potassium channel blocker (TEA) ${ }^{14}$. However, the effect of this $\alpha_{2}$-adrenergic agonist is restored, in either case, when the concentration of calcium is lowered, suggesting the primary involvement of calcium ions ${ }^{14,422}$. The participation of a voltage-sensitive calcium channel is not obvious, however, since $\alpha_{2}$-adrenoceptors still mediate an inhibition of $[3 \mathrm{H}] \mathrm{NA}$ release from preloaded slices of hippocampus in absence of extracellularcalciu. . 229 . Nevertheless, ruthenium red, which blocks the entry of calcium into intracellular stores, decreases the effect of clonidine. Based on these results, Jackisch and co-workers ${ }^{229}$ suggested that the inhibition of adenylate cyclase by $\alpha_{2}$-adrenergic autoreceptors decreases cytoplasmic calcium affecting NA release by primarily acting on the intracellular pool of calcium.

\subsection{Postsynaptic mechanisms}

\subsubsection{Single-unit studies}

Both excitatory and inhibitory effects of the application of NA on the firing rate of pyramidal neurons of hippocampus have been reported $102,287,318,319,342$. The most prominent in vivo action of pressure ejected NA on pyramidal neurons is a suppression of firing which is readily blocked by the $\alpha_{1}$ adrenergic antagonist prazosin, but not by the $\alpha 2$-adrenergic antagonist rauwolscin ${ }^{342}$. In contrast, the suppression of firing activity induced by microiontophoretic application of NA is weakly blocked by prazosin, but almost completely blocked by the $\alpha_{2}$-adrenergic antagonists idazoxan and piperoxan ${ }^{103}$. The above results suggested the existence of twodistinct $\alpha$-adrenoceptors mediating the inhibitory effect of NA. A differential role of these receptors has subsequently been suggested by Curet and de Montigny 103 who used the technique of electrical stimulation of the ascending NA pathway arising from the LC coupled with orthodromic recording of $\mathrm{CA} 3$ pyramidal neurons. Following $\mathrm{LC}$ stimulation, synaptically released NA produces a suppressing effect on the firing rate of pyramidal neurons of hippocampus 397 that is blocked by prazosin, but not by idazoxan ${ }^{102}$. It was thereby concluded that postsynaptic $\alpha_{1}$-adrenoceptors are localized intrasynaptically since they directly respond to synaptically released NA from terminals. In microiontophoresis studies, diffusion of NA through the glass micropipette would rather activate postsynaptic $\alpha 2$-adrenoceptors localized extrasynaptically.

Electrical stimulation of the LC ascending pathway, similarly to pressure ejection of NA, was shown to produce a period of activation that follows the inhibition of the firing rate ${ }^{102,319}$. This excitatory effect of NA on the activity of hippocampal pyramidal neurons was shown to be mediated by $\beta$-adrenoceptors since it was blocked by antagonists such as propanolol ${ }^{102,319}$. The mechanism involved in the excitation of hippocampal pyramidal neurons by $\beta$-adrenoceptors is well established. it implicates the calcium-sensitive voltage-independent potassium current (IAFP) which is implicated in the accommodation of spike firing. By inactivating the channels mediating this potassium current, NA decreases the hyperpolarization that occurs after an action potential and thereby facilitates the generation of new spikes 185,279 . The receptors mediating the blockade of IAHP by NA were shown to be of the $\beta_{1}$ - 
adrenergic subtype as the effect of NA was blocked by the antagonist atenolol and mimicked by the agonist dobutamine ${ }^{281}$. In addition, the second messenger mediating the depolarization and the increase in the firing activity of pyramidal neurons was identified as CAMP since this molecule as well as agents that increase its intracellular availability (forskolin and phosphodiesterase inhibitor) produce excitatory effects similar to those of $\mathrm{NA}^{280}$.

In comparison, there are few intracellular studies that have indicated an action of $\alpha$-adrenoceptors at the level of pyramidal neurons of the hippocampus. A small hyperpolarization of these cells, preceding the $\beta$-adrenergic depolarization, can be induced by $\mathrm{NA}^{261,281}$. Madison and Nicoll 281 assumed this hyperpolarization to be mediated by $\alpha$-adrenoceptors although they admitted that the characterization of thisresponse was problematic. Furthermore, Segal ${ }^{399}$ indicated thatneitherclonidine nor phenylephrine exerted any marked hyperpolarizing action at the level of pyramidal neurons of the hippocampus.

The interactions between the membrane hyperpolarization and the $\beta_{1}$-adrenoceptor-mediated blockade of $I_{\text {AHP }}$ contribute to a prominent function of NA action in the brain, i.e., the modulation of the signal-to-noise ratio. This function of the NA system is iliustrated by the following intracellular experiment performed on hippocampal neurons (see fig. 4) 281 . In a control condition, a brief electrical stimulation of a pyramidal cell was adjusted to a level just above the threshold to elicit a single action potential. A second longer electrical stimulation was carried out to elicit several action potentials with the usual accommodation of firing rate due to the activation of the calcium-dependent potassium current (IAHP). Application of NA to a pyramidal cell reduced its IAHP-mediated accommodation and allowed the cell to fire action potentials throughout the period of the longer electrical stimulation. However, the concomitant hyperpolarizing action of NA blocked the ability of the brief electrical stimulation to elicit a single action potential. It thus appears that NA can suppress responses of pyramidal neurons to noise, i.e. stimuli near the action potential threshold, while at the same time increasing responses to significant signals, i.e. larger stimuli281.

\subsubsection{Multi-unit studies}

When the action of NA was studied at the level of a population of neurons of the $\mathrm{CA}_{1}$ region of the hippocampus, using the technique of field potential recording, biphasic effects were observed, in keeping with the data derived from single unit studies reviewed above. On the one hand, low

control

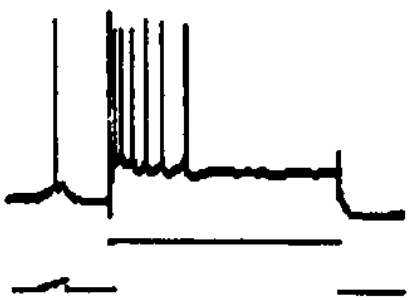

noradrenaline

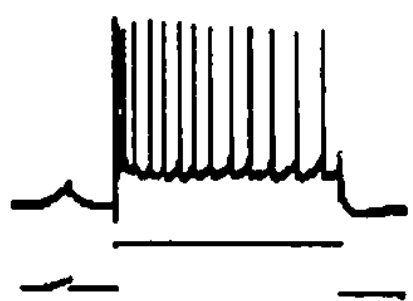

wash

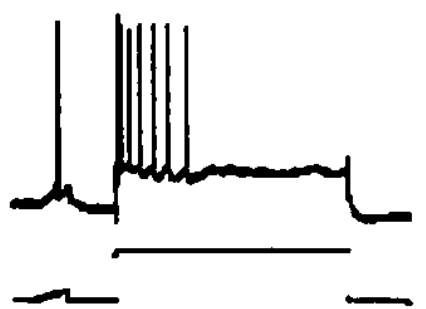

Fig. 4. Experiment illustrating the modulation of the signal-to-noise ratio by NA (modified from Nicoll et al., Psychopharmacology: The third generation of progress, ed. H.Y. Meltzer, Raven press, N.Y., p. 108, 1987) 
concentrations of NA or microiontophoresis of NA onto the cell body layer enhanced the population spike amplitude evoked by Schaffer collateral stimulation. The response was blocked by $\beta$-adrenergic antagonists and mimicked by $\beta$-adrenergic agonists as well as by cAMP analogues $287,317,319$. On the other hand, higher concentrations of NA or phenylephrine suppressed the evoked population spike. The latter response was characterized as being of the $\alpha_{1}$-adrenergic subtype, although clonidine had a similar inhibitory effect at high concentrations $317,319,320$. It is also noteworthy that NA can increase the spontaneous firing rate of interneurons, which can in turn lead to an inhibition of principal cells and a reduction of the population spike amplitude 338 .

The effects of activating $\alpha$-adrenoceptors in the principal cell layers of the hippocampus are not always inhibitory. Indeed, NA was shown to act through $\alpha$-adrenoceptors to reduce the inhibitory action of GABAergic interneurons on the firing activity of pyramidal neurons and thereby increase the excitability of these cells 266,282 . More precisely, NA would reduce the early IPSPs mediated by GABAA receptors that increase chloride influx into pyramidal neurons ${ }^{118}$. However, the cellular site responsible for the disinhibitory effect of NA does not appear to reside at an interneuron. Instead, activation of $\alpha$ adrenoceptors would reduce the GABAergic inhibition by depressing excitatory synapses originating from pyramidal neurons that activate interneurons (recurrent feedbacks) ${ }^{118}$. Another way by which $\alpha_{1-}$ adrenoceptors activation can lead to increase excitability of pyramidal cells is by a potentiation of glutamatergic input mediated by NMDA receptors ${ }^{399}$. $\alpha_{1}$-Adrenoceptors are well known to activate the phosphatidyl inositol pathway in a variety of tissues ${ }^{329}$. These receptors induce the enzymatic hydrolysis of phosphatidyl-inositol-4,5-biphosphate (PIP2) by phospholipase $\mathrm{C}$ which produces diacylglycerol (DAG) and inositol-1,4,5-triphosphate (IP3). The formation of the latter $\mathrm{IP}_{3}$ molecule in turn promotes the release of calcium from intracellular stores ${ }^{329}$. Segal and colleagues ${ }^{399}$ found that microiontophoresis of IP3, but not cAMP, produces the same enhancing effect as $\alpha_{1}$-adrenergic agonists. It was thus concluded that $\alpha_{1}$-adrenoceptors can potentiate the NMDA-msdiated influx of intracellular calcium in hippocampal principal neurons 399 .

The excitation of granule cells of the dentate gyrus by the stimulation of the perforant path was shown to be increased following either prolonged microiontophoretic application of $\mathrm{NA}^{472}$ or bath superfusion of $\mathrm{NA}^{108,254,419}$. This long-term change is produced as well as by the activation of the LC ascending pathway using electrical stimulation ${ }^{109,192}$, glutamate ${ }^{194}$, or blockade of somatodendritic $\alpha 2$-adrenergic autoreceptors with idazoxan ${ }^{379}$. Both the population spike amplitude and the firing activity of pyramidal neurons of the $\mathrm{CA}_{1}$ and $\mathrm{CA}_{3}$ regions elicited by stimulating, respectively, the Schaffer collateral or the mossy fibers were similarly shown to be enhanced by NA (for a review see ${ }^{193}$ ). Besides these data, the fact that responses to topical application of glutamate are enhanced by previous bath superfusion of $\mathrm{NA}^{396}$ suggests that noradrenergic outputs enhance glutamatergic neurotransmission in the hippocampus. These potentiating effects of NA on glutamatergic neurotransmission denominated "NA Long-Lasting Potentiations" (NALLPs) can last for several minutes to several hours, and are thought to be physiologically relevant to memory processes 193 . NALLPs are known to be mediated by $\beta$-adrenergic receptors coupled to the adenylate cyclase system ${ }^{419,420}$. The crucial event triggered by 
the cAMP signal might well be an increased cellular influx of calcium, considering that $\beta$-adrenoceptors were sho:vn to activate voltage-sensitive calcium channels ${ }^{180}$. One of these calcium conductances could be the NMDA receptor-channel because antagonists at this site were shown to suppress the induction of NALLPs 78,418 .

The phenomenon of NALLP is distinct from that of LTP as it does not require high frequency stimulation of excitatory pathways to be induced. However, NALLP appears related to LTP, considering that both phenomena are similarly blocked by protein synthesis inhibitors 420 and that LTP is facilitated by bath applications of $\mathrm{NA}^{216,417}$ but markedly reduced by noradrenergic fiber denervation ${ }^{419,421}$. Furthermore, superfusion of hippocampal slices with a low concentration of NA, insufficient to produce NALLP, paired with a subthreshold high-frequency stimulation of mossy fibers that does not induce by itself LTP, can produce long-lasting potentiation of pyramidal neurons' excitability 215.

\section{Efiects of antidepressant treatments on 5-HT and NA neurons}

\subsection{Tricyclic antidepressant drugs}

Some tricyclic antidepressant drugs (TCAs) decrease the firing rate of DR 5-HT neurons when administered acutely. In this regard, chlorimipramine is quite potent 392,447 , whereas imipramine and amitryptiline inhibit 5-HT neurons' discharges only at relatively higher doses 392 . The inhibitory effect of these drugs correlate with their capacity to block 5-HT reuptake in vitro (chlorimipramine > imipramine > amitriptyline), and TCAs that preferentially block NA reuptake (nortriptyline or desipramine) are either very weak or inactive 356,392 . Following long-term administration of imipramine, the firing activity of 5-HT neurons and the sensitivity of their somatodendritic 5-HT1A autoreceptors to microiontophoretically-applied 5-HT or LSD remained normal 47 . Furthermore, the binding of [3H]8OH-DPAT was found unchanged in the DR area following long-term amitriptyline treatments ${ }^{463}$. 'The fact that these TCAs are rapidly metabolized to secondary amines that are weak 5-HT reuptake blockers has been invoked to explain their lack of long-term effect on the sensitivity of 5-HT1A autoreceptors following sustained administration 57 .

Most TCAs studied so far were found to inhibit the firing rate of LC NA neurons when administered acutely $392,430,432$. The efficacy that these TCAs display in inhibiting the firing activity of NA neurons can also be explained in terms of reuptake blocker potency. Desipramine and nortriptyline are effective at low doses, whereas imipramine and amitriptyline are much less potent ${ }^{392}$. Intraperitoneal administration of desipramine for one to seven days was found to produce small reductions of the firing activity of LC NA neurons 225,298 . However, long-term desipramine treatment of two to three weeks induced a greater decrement of neuronal firing 225 . A two-week treatment with imipramine was also reported to decrease LCNA neurons' firing activity 430,432 . It is noteworthy, however, that these findings could not be replicated by other investigators 255,444 .

Changes in LC NA neurons' activity following long-term TCA treatments would occur in conjunction with other changes at the enzymatic, receptor and second messenger levels. As discussed earlier (section 3.2), the firing rate of NA neurons is directly proportional to the intracellular 
concentration of CAMP. TCA treatments could thus decrease neuronal firing through a change in cAMP concentration in LCNA neurons. In keeping with this view, long-term imipramine was shown to reduce cAMP-dependent protein kinase activity in the LC area ${ }^{300}$. These changes in CAMP levels after TCA treatments might also regulate the expression of proteins in NA neurons. Using immunohistochemistry and in situ hybridization techniques, long-term imipramine and nortriptyline treatments were shown to decrease the expression of tyrosine hydroxylase in the $\mathrm{LC}$ area ${ }^{72,300,323}$. Long-term treatments with imipramine, chlorimipramine and desipramine were also shown to decrease the concentration of $\mathrm{G}_{\alpha \mathrm{s}}$ proteins and increase that of $\mathrm{G}_{\alpha o}$ proteins in the $\mathrm{LC}$ area ${ }^{265}$. Either of these changes could decrease the firing rate of NA neurons either by enhancing the potassium conductance presumably coupled to $\mathrm{G}_{\alpha_{0}}$ or by decreasing the $\mathrm{G}_{\alpha s-c o u p l e d}$ adenylate cyclase production of cAMP265.

The $\alpha 2$-adrenergic autoreceptors mediating the inhibitory feedback on the firing activity of NA neurons might become desensitized following long-term TCA treatments. Indeed, the suppressant effects of intravenous administration of imipramine, desipramine or clonidine, as well as that of microiontophoretically-applied clonidine, were reduced in rats treated with desipramine or imipramine $255,393,432,444$. In agreement with these electrophysiological findings, [3H]clonidine and [3H]idazoxan binding was found to be reduced in the $\mathrm{LC}$ region following long-term amitrityline and protriptyline treatments 251,410 . Curiously enough, the inhibitory effect of NA applied by microiontophoresis remained unchanged after long-term desipramine ${ }^{255}$. As stated earlier (section 2.2), clonidine has some affinity for imidazoline receptors. It is thus possible that it is these imidazoline receptors, rather than the $\alpha 2$-adrenoceptors, that become desensitized to the inhibitory action of clonidine following long-term TCA treatments. In addition, the blunted effect of desipramine and imipramine after these treatments might be explained by the desensitization of NA reuptake carriers 33 . Therefore, the issue regarding the sensitivity of somatodendritic autoreceptors in the LC area after longterm TCAs still remains unclear.

\subsection{Electroconvulsive treatments}

Considering the therapeutic and heuristic importance of electroconvulsive therapy (ECS) for major depression, it is surprising how few studies examined its effect on the physiology of NA and 5HT neurons. One study 433 reported a decreased responsiveness of somatodendritic $\alpha 2$-adrenergic autoreceptors of LCNA neurons to the inhibitory effect of intravenous clonidine after repeated ECS. However, this change was also observed with a single electroconvulsive shock ${ }^{433}$ which does not produce antidepressant effects in humans. Similarly to long-term TCA treatments, ECS was also shown to decrease tyrosine hydroxylase immunoreactivity in the $\mathrm{LC}$ area323.

The effect of ECS on 5-HT neurons of the DR was only recently investigated ${ }^{42}$. Here again, a lack of effect of ECS was observed, as was found after long-term TCA treatments. Furthermore ECS was shown not to change the firing activity of these neurons nor the responsiveness of 5-HT $1 \mathrm{~A}$ autoreceptors to the microiontophoretic application of 5-HT or 8-OH-DPAT ${ }^{42}$. 


\subsection{Monoamine oxidase inhibitors}

A marked decrease in the firing activity of LCNA neirons was observed after a two-day treatment with type A MAOIs such as clorgyline and phenelzine, but not with the type B MAOI deprenyl 50,55 . When the inhibition of MAO A was prolonged to one or three weeks, there was no tendency of NA neurons' firing activity to return to the baseline ${ }^{50,55}$. These results suggest that the regulation of LCNA neurons' firing activity via somatodendritic $\alpha 2$-adrenergic autoreceptors is not altered by long-term MAOIs. Consistent with this view, the suppressant effect of intravenous clonidine on the firing rate of NA neurons was unchanged after these treatments ${ }^{50}$. However, long-term treatments with either clorgyline or phenelzine were found to decrease [3H]clonidine or [3H]idazoxan binding in the LC area 95,251 . This discrepancy could be explained by the fact that there is a particularly large $\alpha 2$-adrenergic receptor reserve on LCNA neurons ${ }^{130}$. Therefore, the small down-regulation of $L C \alpha 2$-adrenoceptors observed in binding studies 95,251 might not be sufficient to affect the $\alpha_{2}$-adrenergic autoinhibitory functions which would ultimately depend upon the ratio of receptors versus $G$ proteins. Other interesting data indicate that phenelzine increases tyrosine hydroxylase mRNA levels ${ }^{71}$. This is the opposite of TCAs which, as reviewed in section 7.1, decrease the expression of this enzyme 72 .

Similarly to NA neurons, DR 5-HT neurons were shown to reduce their firing rate in response to two-day treatments with MAOIs of type $A^{50,55}$. In contrast with NA neurons however, 5-HT neurons firing activity displays a partial recovery after one week and a complete one after three weeks of treatment ${ }^{50,55}$ (see fig. 5). The gradual recovery of 5-HT neuronal discharge was accounted for by the finding that 5-HT1A autoreceptors become desensitized following long-term MAOIs. Indeed, the inhibitory effect of intravenous LSD was markedly reduced following long-term clorgyline, phenelzine or amiflamine treatments 50,55 . Unfortunately, there are actually no biochemical correlates of these electrophysiological findings. In one autoradiographic study, sustained MAO inhibition with clorgyline, phenelzine or tranylcypromine failed to decrease the binding of [3H]8-OH-DPAT in the MR and DR
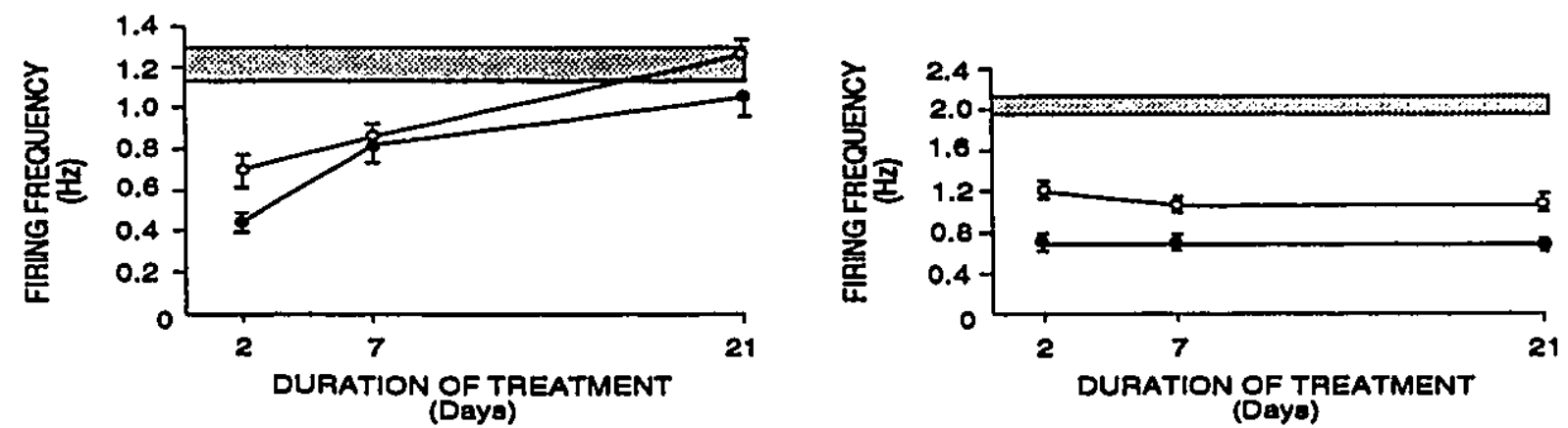

$\bullet$ CLORGYLINE ( $1 \mathrm{mg} / \mathrm{kg} / \mathrm{day})$

○ PHENELZINE (2.6 mg/kg/day)

Fig. 5. Alterations of 5-HT (left) and NA (right) neurons' firing activity by MAOI treatments (modified from Blier and de Montigny, Neuroscience, 16,949; 1985 with the permission of authors) 
$\operatorname{areas}^{206}$. As discussed earlier, 5-HT1A autoreceptors are coupled with $\mathrm{Gi}_{\mathrm{i} / \mathrm{o}}$ proteins 60,228 . The desensitization of the 5-HT1A-mediated autoinhibition possibly results from an alteration at the level of $\mathrm{G}$ protein functions rather than from a down-regulation of autoreceptors.

\subsection{Selective reuptake blockers}

Similarly to most TCAs, acute intravenous administration of the selective serotonin reuptake inhibitors (SSRIs) citalopram, indalpine or zimelidine dose-dependently inhibits the firing activity of DR 5-HT neurons, but at much lower doses 58,89,112. Furthermore, these drugs were shown to markedly decrease the firing activity of 5 -HT neurons after two-day treatments $48,58,89$. There was a partial recovery after one week and a complete one after three weeks of treatment. Interestingly, these changes in firing activity after long-term SSRIs were associated with subseisitivity of the somatodendritic 5-HT autoreceptors. Indeed, the dose-response curve for the inhibitory effect of LSD on the firing activity of 5-HT neurons was found shifted to the right after long-term citalopram 89 and the effect of $10 \mu \mathrm{g} / \mathrm{kg}$ of LSD was markedly reduced in rats treated for two-weeks with indalpine or zimelidine 48,58 . The recovery of 5-HT neuron firing activity after long-term SSRIs can be explained by the desensitization of the somatodendritic 5-HT autoreceptors and might account, at least in part, for the delayed efficacy of thise agents in depression.

There are few biochemical studies that have investigated the effect of long-term SSRI treatments on 5-HT1A receptor binding in the raphe regions. There is one report indicating a decreased $[3 \mathrm{H}] 8-\mathrm{OH}$ DPAT binding sites in the DR area following long-term treatments with the SSRI fluoxetine ${ }^{463}$. Another study, however, did not indicate any change in [3H]8-OH-DPAT binding in both the MR and DR regions after long-term 5-HT reuptake inhibition with citalopram or sertraline 206.

Selective NA reuptake blockers such as oxaprotiline or maprotiline have not been investigated for their long-term effects on the physiology of NA neurons. Nevertheless, long-term treatments with these antidepressant drugs would be expected to have a similar profile of action on the firing activity of LC NA neurons as those described earlier for TCAs having preferential affinities for the NA reuptake carrier such as desipramine or nortriptyline. Long-term SSRI treatments, contrary to TCA or MAOI treatments, did not change $[3 \mathrm{H}]$ idazoxan binding in the $\mathrm{LC}$ area 71 . Surprisingly however, long-term fluoxetine treatments increased the expression of tyrosine hydroxylase in the LC area to the same extent as did longterm MAOI treatments ${ }^{71}$. Furthermore, long-term zimelidine treatments were shown to decrease the responsiveness of somatodendritic $\alpha_{2}$-adrenergic autoreceptors to microiontophoretically-applied clonidine, although the effect of this treatment at this level was considerably smaller than that of desipramine or imipramine 393.

\subsection{Azapirones and ca-adrenergic antagonists}

As stated earlier, the antidepressant drugs of the azapirone family such as buspirone, gepirone, tandospirone or ipsapirone are high affinity ligands for 5-HT $1 \mathrm{~A}$ receptors. It is therefore not surprising that the intravenous administration of the above agents was found to potently decrease tie firing rate of 
DR 5-HT neurons 51,166,391,413,448. Short-term treatments (two-day) with gepirone or tandospirone also produce a decrease in 5-HT neurons firing activity. As for MAOI and SSRI treatments, there is a partial recovery after one week and a complete one after two weeks of treatment with gepirone or taridospirone 53,166 . The recovery of 5-HT neuronal firing was again ascribed to a desensitization of 5$\mathrm{HT}_{1 \mathrm{~A}}$ somatodendritic autoreceptors as the dose-response curve of LSD was shifted to the right and the suppressant effects of microiontophoretically-applied 5-HT, LSD, 8-OH-DPAT or gepirore were markedly reduced after these treatments $51,53,166$. Results consistent with the above interpretation were obtained in experiments using in vitro recording of 5-HT neuron firing activity in brainstem slices as the concentration-dependent inhibition by 8-OH-DPAT of 5-HT neuronal discharges was shifted to the right after a two-week treatment with ipsapirone ${ }^{381}$.

Effects of long-term treatments with gepirone and ipsapirone on the density of somatodendritic 5-HT autoreceptors were investigated using autoradiographic techniques. A three week treatment with gepirone ( $10 \mathrm{mg} / \mathrm{kg} /$ day) was initially shown to produce a small decrease $(-27 \%)$ of [3H]8-OH-DPAT binding in the DR area. However, subsequent studies using the same gepirone treatment, or twice daily injections of ipsapirone ( $5 \mathrm{mg} / \mathrm{kg} /$ day) during two weeks, failed to replicate these results 312,381 . Further studies revealed that stringent conditions are necessary to produce the down-regulation of 5-HT1A receptors with azapirone treatments. Twice daily injections of ipsapirone (10 mg/kg/day) produced a small decrease $(-20 \%)$ of [3H]8-OH-DPAT labeling in the DR area after a two-week treatment, whereas a three-week treatment with this same drug markedly reduced (-63\%) this binding ${ }^{136}$. However, 5$\mathrm{HT}_{1 \mathrm{~A}}$ receptors in the MR nucleus were found unaffected by the latter ipsapirone treatment ${ }^{136}$. It thus remains unclear whether a down-regulation of 5-HT1A receptors in the raphe area can account for the desensitization of 5-HT $1 \mathrm{~A}$ autoreceptors by azapirone treatments.

The common metabolite of azapirones is 1-(2-pyrimidinyl)-piperazine (1-PP), a potent $\alpha_{2}$ adrenergic antagonist. Acute administration of 1-PP, or parent azapirone compounds, were shown to increase the firing rate of LC NA neurons $46,74,129,376,377$. In this regard, the action of azapirones is similar to that of another antidepressant drug, mianserin, which was also shown to increase NA neurons' activity by blocking somatodendritic $\alpha_{2 \text {-adrenergic autoreceptors }}{ }^{107}$. The selective $\alpha_{2 \text {-adrenergic }}$ antagonist and purported antidepressant drug idazoxan has a complex profile of action at LC NA neurons. Under sodium pentobarbital anesthesia, a low intravenous dose of idazoxan was shown to decrease the firing rate of LCNA neurons whereas microiontophoretic application of this drug increases NA neuronal discharges 152,168 . When chloral hydrate was used instead for anesthesia, the suppressant effect of the low dose of idazoxan was not observed and higher doses of this drug actually increased NA neurons' firing activity 152 .

It can thus be concluded that some antidepressant drugs increase the firing rate of LCNA neurons when given acutely. However, this is unlikely to be the basic property underlying their therapeutic efficacy in depression. First, because it stands in contrast with the effect of TCAs that, as we have seen, decrease neuronal discharge from these cells. And second, because the enhancing effects of tandospirone and mianserin have been found to disappear upon long-term administration 107,166 , yet these drugs are clinically effective at this stage. 


\section{Effects of antidepressant treatments on hippocampai 5-HT and NA systems}

\subsection{At the presynaptic level}

\subsubsection{Tricyclic antidepressant drugs}

The only TCA that has been studied so far for its effect at NA terminals is the NA reuptake blocker desipramine. Althougks it prolongs the time of action of NA in the synaptic cleft, desipramine appears to reduce the amount of NA released per action potential. Acute administration of this drug has been shown to decrease the efficacy of the electrical stimulation of the LC ascending pathway to suppress the firing activity of $\mathrm{CA} 3$ pyramidal neurons, and this was shown to be mediated via the activation of terminal $\alpha_{2}$-adrenergic autoreceptors ${ }^{105}$. A two-week treatment with $5 \mathrm{mg} / \mathrm{kg} /$ day of desipramine did not change the efficacy of the stimulation of the NA pathway. In contrast, a treatment with a higher dose of desipramine (10 mg/kg/day) increased the efficacy of the electrical stimulation at $5 \mathrm{~Hz}^{255}$. The latter in vivo result is consistent with previous in vitro experiments that have shown enhanced electrically- or potassium-evoked release of [3H]NA following treatments of three to four weeks with desipramine at a similar regimen 81,388 . The enhanced release of NA following desipramine treatment could be ascribed to a desensitization of terminal $\alpha_{2}$-adrenergic autoreceptors. In support of this contention is the fact that the inhibitory effect of intravenous clonidine on the efficacy of the stimulation of NA pathway is reduced following long-term desipramine treatments $(10 \mathrm{mg} / \mathrm{kg} / \text { day })^{255}$. Furthermore, the effect of increasing the frequency of the stimulation, on the level of activation of $\alpha_{2}$-adrenergic autoreceptors by synaptically released NA, was also diminished after long-term desipramine treatments ${ }^{255}$. However, two groups found that the inhibitory effect of clonidine on the evoked release of [3H]NA from preloaded hippocampal slices in vitro is unaltered by a similar desipramine treatments ${ }^{81,388}$, thus yet excluding any definitive conclusion.

There is one superfusion study that suggested a decreased inhibitory effect of clonidine after longterm desipramine 214 . However, in these experiments the animals were sacrificed only 12 hours after the last injection of desipramine, and it is possible that elevated levels of endogenous NA, produced by remaining NA reuptake blockade with desipramine, competed with clonidine. Smith et al.410 also reported a decreased density of $[3 \mathrm{H}]$ clonidine binding sites in hippocampus following long-term amitriptyline, and claimed that this effect might be the biochemical correlate of an $\alpha_{2}$-adrenergic autoreceptor desensitization on the basis that this effect is not observed in rats that had NA fiber denervation at birth. This conclusion can be questioned, considering that a desensitization of postsynaptic $\alpha_{2}$-adrenoceptors by an increased synaptic concentration of NA (consequent to the sustained reuptake blockade produced by amitriptyline) would also be prevented by NA fiber denervation. In subsequent studies 214 , the same group reported decrease in [3H]clonidine binding after long-term desipramine treatment which is consistent with the above results with amitriptyline.

There are two studies that have investigated the effect of long-term TCA treatments on 5-HT terminals of the hippocampus. A three-week treatment with imipramine was shown to increase the efficacy of the electrical stimulation of the 5-HT pathway to suppress the firing activity of CA3 pyramidal 
neurons 56,90 . However, a desensitization of terminal 5-HT1B autoreceptors is not likely to account for this eftect: first, because the efficacy of the 5-Н $\mathrm{H}_{1 \mathrm{~B}}$ antagonist methiothepin to increase the suppression of firing was unchanged, and second, because the difference in the degree of suppression of firing observed by increasing the frequency of the stimulation was also unaltered 56,90 .

\subsubsection{Electroconvulsive shock treatment}

There are few studies that have investigated the effect of ECS on NA or 5-HT terminals of the hippocampus. Acutely, electroconvulsive shocks increase the release of various neurotransmitters, including NA and 5-HT, in the hippocampus, ${ }^{125,132,434}$. However, microdialysis andelectrophysiological studies have shown that ECS does not produce a sustained increase the release of NA nor of 5-HT in the hippocampus 90,434 . Furthermore, these studies indicated unchanged sensitivity of NA or 5-HT terminal autoreceptors as assessed by the unaltered enhancing effects of antagonists, idazoxan and methiothepin respectively, acting at these sites 90,434 . Considering these functional data, the decreased [3H]clonidine binding that has been reported in the hippocampus after ECS ${ }^{415}$ can hardly be ascribed to a down-regulation of $\alpha_{2}$-adrenoceptors on NA terminals. Finally, there is one report indicating that the funtion of the 5-HT3 receptors that enhance the evoked release of [3H]5-HT is not modified by $\mathrm{ECS}^{42}$.

\subsubsection{Monoamine oxidase inhibitors}

Brain concentrations of 5-HT and NA have been found elevated after both short- and long-term treatments with various MAOIs ${ }^{54,55,144}$. These results, however, are not necessarily indicating that an increased amount of neurotransmitters is available for release following neuronal depolarization. In fact, increased cytoplasmic levels of 5-HT and NA appear to inhibit the synthesizing enzymes of these neurotransmitters and could thus limit their intraneuronal accumulation and terminal release ${ }^{144}$. Consistent with this interpretation is the fact that long-term inhibition of MAO A with clorgyline or phenelzine does not change the efficacy of the stimulation of the ascending NA pathway to suppress the firing activity of pyramidal neurons of hippocampus ${ }^{54}$. Finberg and co-workers documented a desensitization of prejunctional $\alpha_{2}$-adrenergic autoreceptors in the periphery following long-term MAOIs ${ }^{142,143}$. However, terminal $\alpha_{2}$-adrenergic autoreceptors of the central nervous system are apparently not affected by these treatments 81,341 .

In contrast to NA, 5-HT neurotransmission was found to be enhanced following long-term treatments with MAOIs of $A$ type $45,54,55$. This phenomenon is most likely explained by a presynaptic mechanism because the sensitivity of postsynaptic 5-HT receptors was not increased by these treatments $45,54,55$. A down-regulation of presynaptic terminal 5-HT autoreceptors is an appealing explanation for the increased release of 5-HT, considering that long-term treatments with MAOIs have consistently been shown to decrease [3H]5-HT binding to 5-HT1 receptors in the brain274,349,380. However, selective lesions of inputs from the raphe to the hippocampus (administration of 5,7dihydroxytryptamine in the cingulum and the fimbria fornix) do not cause any significant decrease in 
[3H]5-HT binding in the hippocampus 355 , thus indicating that these binding sites are postsynaptic. In the light of this evidence, the down-regulation of [3H]5-HT binding sites by MAOIs does not appear relevant to an action of these treatments at presynaptic sites. Furthermore, an electrophysiological study by Blier et al. ${ }^{45}$ ruled out a possible desensitization of terminal 5-HT autoreceptors of hippocampus by long-term clorgyline. Indeed, the enhancing effect of methiothepin and the ratio of effectiveness of two frequencies of stimulation were found to be unchanged after this treatment ${ }^{45}$. It is not clear at present if other factors, in addition to the increase in the releasable pool of 5-HT, contribute to the increased 5HT neurotransmission after long-term MAOI treatments.

\subsubsection{Selective reuptake inhibitors}

No studies have investigated the effect of antidepressant drugs with selective NA reuptake inhibitor properties, apart from that of the TCA desipramine, on the function of noradrenergic terminals of the hippocampus. In comparison, the actions of SSRIs at 5-HT terminals have been the subject of numerous experiments. We have seen (section 5.1) that bath application of paroxetine in vitro increases the evoked the release of [3H]5-HT from preloaded hippocampal slices through 5-HT3 receptors ${ }^{43}$. Interestingly, long-term treatment with parox.tine was recently shown to blunt the enhancing effect of the selective 5-HT3 agonist 2-methyl-5-HT4 . It is noteworthy that a mere increase in the synaptic concentration of 5-HT during the paroxetine treatment is not sufficient to explain the above data inasmuch as long-term treatments with a MAOI do not produce this effect ${ }^{44}$.

It appears nonetheless that, similarly to MAOIs, various SSRIs (citalopram, zimelidine, fluoxetine and paroxetine) increase the efficacy of 5-HT neurotransmission in vivo through a presynaptic mechanism ${ }^{45,48,89,90}$. However, in contrast to the results obtained with MAOIs, SSRIs were consistently shown to decrease the sensitivity of terminal 5-HT autoreceptors in the hippocampus. Indeed, the enhancing effect of methiothepin on the efficacy of the electrical stimulation of the 5-HT pathway to suppress the firing rate of pyramidal neurons was nearly abolished by long-term paroxetine, fluoxetine or citalopram treatments (fig. 6). In addition, increasing the frequency of the stimulation did not decrease the efficacy of 5-HT neurotransmission in these treated animals to the same extent as in saline-treated rats ${ }^{45,89,90}$. Experiments carried out in vitro on brain slices provided further evidence consistent with the desensitization of terminal 5-ETT autoreceptors. Long-term treatments with paroxetine were shown to increase the electrically-evoked release of [3H]5-HT from preloaded hippocampal slices, and to shift to the right the concentration-effect curve of the 5HTIB autoreceptor agonist 5-CT 44 . Finally, it is noteworthy

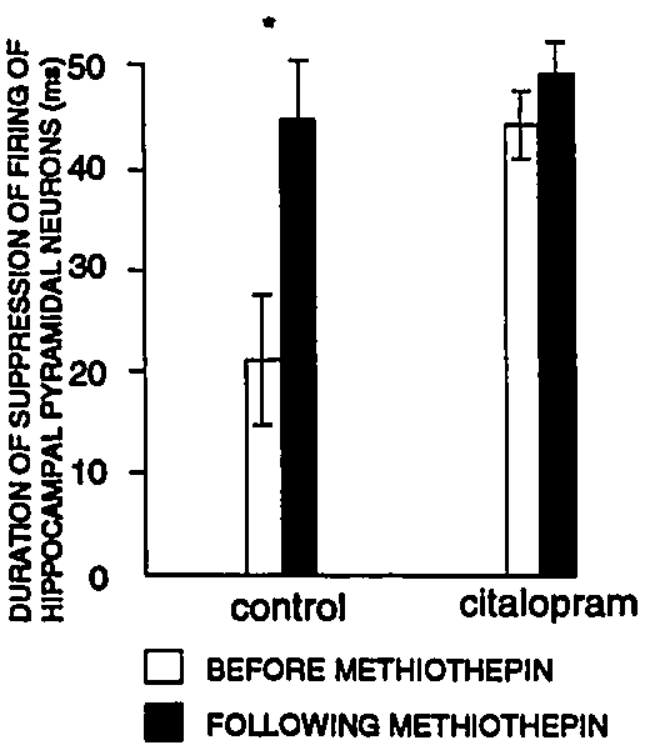

Fig. 6 Effect of a long-term citalopram treatment on 5-HT neurotransmission (modified from Chaput et al, Adv. Biol. Psych. 17,$1 ; 1988$ with the permission of authors) 
that the desensitization of terminal 5-HT autoreceptors by SSRIs is not restricted to the hippocampus as it was also found in the hypothalamus 44,315 .

\subsubsection{Azapirones and $\alpha_{2}$-adrenergic antagonists}

Gepirone and tandospirone have been studied for their effects on the function of 5-HT and NA terminals. Long-term treatments with these drugs do not change the efficacy of 5-HT or NA neurotransmission, as suggested by the unchanged suppressant effects on the firing activity of $\mathrm{CA}_{3}$ pyramidal neurons of electrically stimulating 5-HT and NA ascending pathways $46,51,166$. Furthermore, the ratio of duration of suppression of firing at two frequencies of stimulation was found to be unchanged, indicating that terminal autoreceptors on 5-HT and NA terminals remained normosensitive after longterm azapirone treatments $46,51,166$.

Similarly to mianserin and idazoxan, the metabolite of azapirones, 1-PP, increases the release of NA through the blockade of terminal $\alpha 2$-adrenergic autoreceptors $46,104,242$. Although the above agents are clinically effective after long-term administration, it is possible that this acute property contributes to their therapeutic action. Long-term $\alpha_{2}$-adrenoceptor antagonism with mianserin and idazoxan has been shown to sensitize terminal NA autoreceptors, and this was detectable two to three days after the washout of the drugs. Noradrenergic neurons thus seem to compensate, through the bias of this mechanism, for the large increase in NA release produced by the sustained blockade of terminal autoreceptors. It is likely that 1-PP produced a similar effect although it was not observed in the study by Blier et al. 46 , probably because the osmotic minipumps delivering tandospirone were left in place during the experiments.

\subsection{At the postsynaptic level}

\subsubsection{Serotonergic receptors}

Several single-unit in vivo studies have shown that long-term TCA treatments (chlorimipramine, imipramine, amitriptyline, desipramine) increase the responsiveness of extrasynaptic 5-HT 1 A receptors mediating the inhibition of the firing activity of hippocampus pyramidal neurons. Intrasynaptic 5-HT $1 \mathrm{~A}$ receptors, activated by the eiectrical stimulation of the ascending 5-HT pathway, were also found supersensitive after these TCA treatments $90,111,112,157,178$. Interestingly, this sensitization of 5-HT1A receptors was also produced by repeated ECS treatments, as well as by some atypical antidepressant drugs (iprindole, mianserin), but not by MAOIs, SSRIs or azapirones $57,90,110$. Long-term treatments with MAOIs increase the efficacy of the stimulation of the 5-HT pathway in the hippocampus. However clorgyline, but not other MAOIs, was found to decrease the suppressanteffect of microiontophoreticallyapplied 5-HT and 8-OH-DPAT ${ }^{45,90 .}$

Multi-unit in vitro studies have generated contradictory data concerning the sensitivity of postsynaptic 5-HT receptors following long-term antidepressant treatments. One multi-unit study, consistent with the above single-unit studies, has shown that long-term treatments with desipramine 
enhance the reduction in population spike amplitude induced by bath application of 5-HT ${ }^{117}$. Another group, however, found no significant change of this reduction of population spike amplitude after longterm chlorimipramine ${ }^{336}$. In yet another study, four to five weeks of treatment with imipramine, desipramine or clorgyline was found to decrease the inhibitory effect of 5-HT on the population spike amplitude 372 . However, the inhibitory effect of 5-HT in the latter study appeared to be mediated by $5-$ $\mathrm{HT}_{2}$ receptors as it was blocked by cyproheptadine and ketanserin 372 .

There are also controversies concerning the effect of long-term antidepressant treatments on the 5-HT1A-mediated inhibition of forskolin-stimulated adenylate cyclase in the hippocampus. Newman and co-workers reported that this function is desensitized by a variety of long-term treatments including a TCA (desipramine), ECS, SSRIs (fluoxetine, zimelidine) and even a selective NA reuptake blocker (maprotiline) $324,325,328$. Prolonged administration of low dnses of azapirones (buspirone, ipsapirone) produce similar effects, but curiously, a single administration of a higher dose of buspirone also desensitized the inhibitory effect of 5-HT on adenylate cyclase activity ${ }^{328}$. Two groups found that longterm treatments with MAOIs of type A (tranylcypromine or clorgyline) decrease the 5-HT1A-mediated inhibition of cAMP production ${ }^{409,449}$. Although Varrault and colleagues ${ }^{449}$ also observed a desensitization following ECS, they did not observe any change after long-term fluoxetine or desipramine treatments. Finally, one group reported no alterations in the 5-HT1A-mediated inhibition of forskolin activated adenylate cyclase following long-term treatments with either the TCAs desipramine and imipramine or the MAOI tranylcypromine 333 . Unfortunately, satisfactory explanations that could account for these discrepancies have not been proposed in any of the above studies. To my knowledge, the effect of long-term antidepressant treatment on the production of phosphatidyl inositol mediated by the activation of 5-HT2 receptors in the hippocampus has never been examined, although it was found to be decreased in the cortex by several antidepressant drugs 326 .

There is a general agreement among various studies that long-term treatments with directly acting 5-HT1A agonists (azapirones) or sustained increase in the synaptic concentration of 5-HT (with SSRIs or MAOIs)do notchange the density or affinity of 5-HT 1A receptors in the hippocampus 136,206,312,381,463. In the case of long-term TCA treatments, the status of 5-HT1A binding sites is controversial. Welner and co-workers ${ }^{463}$ reported an increased density of [3H]8-OH-DPAT binding sites in the $\mathrm{CA}_{1}$ region and dentate gyrus after long-term treatment with amitriptyline. This would be consistent with the sensitization of 5-HT 1 a receptors by this treatment that was repeatedly reported in electrophysiological experiments. In one study 310 , long-term imipramine was found to down-regulate 5-HT1A receptors, but others suggested that this TCA treatment does not alter 5-HT1A receptor density 312,460 . Furthermore, the decreased binding of [3H]8-OH-DPAT observed in certain regions of the hippocampus after repeated stress was not reversed by long-term imipramine treatments 460 . Finally, a recent study 79 reported an increase in the levels of hippocampal 5-HT1A receptors, but not mRNA, in LEW/N rats treated with imipramine for 8 weeks. However, in F344/N rats that had received a similar treatment, there was a decreased abundance of mRNAs coding for the 5-HT1A receptor while the density of 5-HT1A receptors was unaltered 79 . 
Several studies documented a reduction of 5-HT2 receptor number by long-term antidepressant drugs in several brain areas including the hippocampus. Although there are some negative findings, antidepressant drugs of all classes including TCAs, SSRIs, MAOIs and azapirones, have been reported to down-regulate these sites $371,381,471,480$. Nonetheless, several authors believe that the downregulation of 5-HT2 receptors by antidepressant drugs is not related to their therapeutic activity as repeated ECS, actually a very effective antidepressant treatment, has been consistently shown to produce the opposite change, i.e., it increases 5-HT2 receptor density 471,480 .

Taken together, the above results suggest that antidepressant treatments might have opposite actions on postsynaptic 5-HT-mediated functions depending on the population of receptors and the mechanisms of transduction involved. For example, based on binding and electrophysiological data, it might be argued that TCA treatments desensitize the 5-HT2-mediated function by decreasing receptors density. On the other hand, TCA treatments increase the neurotransmission at the level of the $5-\mathrm{H}^{\mathrm{i}} 1 \mathrm{~A}$ receptors that inhibit the firing rate of pyramidal cells of hippocampus. In contrast to the desensitization of 5-HT2-mediated functions, the TCA-induced enhancement of 5- $\mathrm{HT}_{1 \mathrm{~A}}$-mediated function would take place beyond the receptor itself, first because, no consistent changes were found in [3H]8-OH-DPAT binding studies after these treatments, and second, because TCAs would decrease rather than increase the 5-HT1A-mediated inhibition of adenylate cyclase. Recent studies implicate various subunits of $G$ proteins in the effect of long-term TCA treatments. The findings that long-term TCA treatments increase the brain concentration of $G_{\alpha o}$ proteins and that TCAs directly enhance GTPase activity of $G_{\alpha o}$ proteins could account for the enhancement of the 5-HT1A-mediated electrophysiological response ${ }^{265.476}$. In turn, a reduction in the brain concentration of $\mathrm{G}_{\alpha \mathrm{i}}$ after long-term TCA treatments has also been reported and could explain the diminished capacity of 5-HT to inhibit adenylate cyclase activity 265 .

\subsubsection{Noradrenergic receptors}

Among the better documented postsynaptic effects of long-term antidepressant treatments is the desensitization of $\beta$-adrenoceptors. In contrast to the results obtained for postsynaptic 5-HT receptors, there is reasonable agreement among electrophysiological, second messenger and binding studies. As reviewed earlier (section 6.2), stimulation of the ascending pathways from the LC produce, through synaptically released NA, a $\beta$-adrenergic-mediated activation of the firing activity of pyramidal neurons of hippocampus. This response is decreased following long-term treatment with desipramine ${ }^{255}$. Longterm treatments with desipramine, mianserin and imipramine were also shown to depress the excitatory action of the $\beta$-adrenergic agonist isoprenaline on the population spike amplitude 117 and decrease the excitatory effect of NA on the firing rate of $\mathrm{CA}_{1}$ hippocampal neurons ${ }^{40}$. In addition, intracellular recordings revealed a marked reduction in the concentration-response curve of isoproterenol, to decrease the afterhyperpolarization amplitude of pyramidal neurons, following long-term imipramine administration 39 . 
A large variety of antidepressant treatments, including TCAs, MAOIs, ECS and atypical antidepressant drugs (e.g., iprindole, mianserin) have been shown to reduce either $\beta$-adrenoceptor binding or $\beta$-adrenergic-mediated production of cAMP326,428. Contrary to the original belief, not all antidepressant drugs have this property. Citalopram and trimipramine do not exert this effect 198,227 . Furthermore, most experiments that have investigated the desensitization of $\beta$-adrenoceptors by antidepressant treatments used brain samples from the cortex. In the hippocampus, as in the cortex, ECS and long-term treatment with TCAs (desipramine, imipramine) or with a MAOI (pargyline) were shown to down-regulate [3H]dihydroalprenolol binding 77,246,345,414-416. Surprisingly, long-term treatment with amitryptiline, nomifensine or tranylcypromine down-regulated the $\beta$-adrenoceptors of the cortex, but not those of the hippocampus 345,416 . Therefore, although the desensitization of $\beta$-adrenoceptors is a shared effect of numerous antidepressant treatments in the cortex, this does not appear to be the case in other areas, such as the hippocampus. Furthermore, there are regional variations in the desensitization of the $\beta$-adrenoceptors even within the hippocampus. Long-term imipramine treatments reduced [125]pindolol binding in the $\mathrm{CA}_{1}$ stratum radiatum or the molecular layer of the dendate gyrus, but not in the $\mathrm{CA} 3$ stratum radiatum ${ }^{123}$. Interestingly, Paul and co-workers ${ }^{345}$ reported that the kinetics of the desensitization of $\beta$-adrenoceptors are more rapid in the frontal cortex than in the hippocampus. They have also observed that in the hippocampus, but not in the cortex, this kinetic effect is accelerated by stress.

The desensitization of $\beta$-adrenoceptors appears to be an adaptative mechanism in response to the enhancement in the synaptic concentration of NA produced by antidepressant drugs. Indeed, 6-OHDA denervation of NA fibers or $\beta$-adrenoceptor blockade abolished the effect of long-term desipramine treatments on cAMP stimulation ${ }^{339}$ and on $\beta$-adrenergic binding 390,473 . ECS apparently desensitizes $\beta$-adrenoceptors through a different mechanism because the effect of this treatment was found to be unaltered in NA-denervated animals 453 . There are several lines of evidence indicating that $\beta$ adrenoceptors desensitize very rapidly in response to short-term agonist exposure, a phenomenon involving phosphorylation of the receptors by protein kinases (PKA or $\beta A R K)^{197}$. However, the desensitization of $\beta$-adrenoceptors by long-term antidepressant treatments cannot be explained by a phosphorylation mechanism ${ }^{197}$. Instead, a decreased production of mRNA coding for $\beta$-adrenoceptors has been reported following long-term antidepressant treatments ${ }^{217}$. The desensitization of the $\beta$ adrenergic-mediated production of CAMP by antidepressant treatments might also involve an altered capacity of $G_{\alpha s}$ proteins to stimulate adenylate cyclase. Similarly to cholera toxin, long-term desipramine or amitriptyline treatments appear to increase the coupling of $\mathbf{G}_{\alpha s}$ to adenylate cyclase and would thereby reduce the capacity of $\beta$-adrenoceptors to stimulate cAMP production by the intermediary of this nucleotide 265,340 .

Some data suggest an enhancement of $\alpha_{1}$-adrenergic responses in hippocampus following longterm antidepressant treatments. A two-week administration of imipramine, mianserin and oxaprotiline increased the suppressant effect of phenylephrine on the firing rate of $\mathrm{CA}_{1}$ hippocampal neurons in vitro ${ }^{40}$. However, treatment with MAOIs, desipramine or tandospirone did not change the efficacy of 
the electrical stimulation (at $1 \mathrm{~Hz}$ ) of the $\mathrm{LC}$ pathway to suppress the firing activity of $\mathrm{CA} 3$ pyramidal neurons, an effect which is mediated through $\alpha_{1}$-adrenoceptors $46,54,55,255$. The above results of Bijak ${ }^{40}$ appear consistent with the enhancement of affinity and sensitivity of brain $\alpha_{1 \text {-adrenoceptors following }}$ long-term antidepressant drugs that Aghajanian and co-workers found in other regions of the brain 302 304.458. There is also one report indicating that the binding of [3H]WB-4101 to $\alpha_{1}$-adrenoceptor of mouse hippocampal membrane is increased by a long-term amitriptylire treatment ${ }^{363}$. In contrast, the binding of $[3 \mathrm{H}]$ prazosin to these receptors in the rat hippocampus was found to be unaltered by longterm amitriptyline, imipramine os mianserin treatments ${ }^{199,332}$. Furthermore, NA-stimulated inositol phospholipid breakdown via $\alpha_{1}$-adrenoceptors was tound unchanged following long-term desipramine, mianserin or maprotiline treatments ${ }^{146}$. And although repeated ECS consistently increases the density of $[3 \mathrm{H}]$ prazosin binding sites and the $\alpha_{1}$-adrenergic stimulation of inositol phosphate in the cortex, no changes were found in the hippocampus after this treatment ${ }^{199,327,332,426}$. The bulk of evidence thus suggests that the $\alpha_{1}$-adrenoceptors of the hippocampus are not generally affected by antidepressant

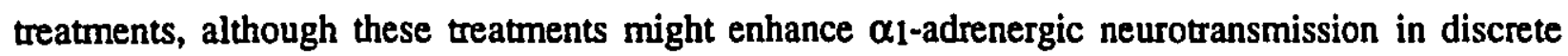
regions of the hippocampus.

Electrophysiological studies have consistently found no change in the sensitivity of postsynaptic $\alpha_{2}$-adrenoceptors to suppress the firing activity of hippocampal pyramidal neurons following long-term antidepressant treatments. The treatments studied were repeated ECS for two weeks ${ }^{110}$, TCAs (desipramine, chlorimipramine, imipramine, amitryptiline) for two days, one week or two weeks ${ }^{111,157,178,212}$, mianserin or indalpine for two weeks ${ }^{212}$, or MAOIs (clorgyline, phenelzine, amiflamine) for two days or three weeks $52,54,55$. These results stand in contrast with the data of $[3 \mathrm{H}]$ clonidine binding studies that indicated a decrease in $\alpha_{2}$-adrenoceptor number in hippocampus following repeated ECS for 10 days 415 or amiriptyline for two weeks ${ }^{32}$. A recent radioligand binding study using $[3 \mathrm{H}]$ clonidine revealed, however, that the down-regulation of $\alpha 2$-adrenoceptors of hippocampus by TCAs is clearly observed after one week of treatment, smaller after two weeks of treatment, and disappears after three weeks of treatment ${ }^{32}$. A similar progressive recovery of [3H]clonidine binding was observed in other regions of the brain ${ }^{32}$. It can thus be reasonably concluded, based on the above electrophysiological data and on the latter binding study, that postsynaptic $\alpha_{2}$ adrenergic functions and receptor number are not affected by long-term antidepressant treatments.

\section{Interactions between the serotonergic and noradrenergic systems}

As described in the preceding sections, there is an abundance of data indicating that the noradrenergic and the serotonergic systems are implicated in the mechanism of action of antidepressant drugs. It might be argued that it is a joint dysfunction of the NA - 5-HT system that is etiologically relevant in depression. The interplay between the modifications induced by antidepressant treatments in both of these systems could also be crucial. There is one study that has indicated a significant correlation between 5-HIAA and MHPG in patients with major depression, suggesting that 5-HT and NA deficits occur in parallel 224 . A more recent study ${ }^{23}$ investigated the relationship between the $\alpha_{1-}$ 
adrenergic and 5-HT neurotransmission using the cortisol response to desipramine and that to the nonselective 5-HT agonist mCPP (meta-chlorophenylpiperazine), respectively. A significant negative correlation between the serotonergic and noradrenergic cortisol responses was found, and interestingly, the magnitude of this correlation was modest in healthy volunteers but highly significant for major depressive patients ${ }^{23}$. From these results, Asnis and his collaborators ${ }^{23}$ concluded a joint disturbance of the NA - 5-HT systems in major depression. There is also clinical evidence that the combinations of antidepressant treatments that increase both NA and 5-HT synaptic concentrations display superior therapeutic efficacy to either one alone. A more rapid antidepressant response was achieved when depressive patints were treated with a 5-HT reuptake blocker (fluoxetine) plus a NA reuptake blocker (desipramine), than when they were treated with desipramine alone ${ }^{322}$. Furthermore, the administration of tyrosine, the precursor of NA, potentiates the antidepressant capacity of 5-HTP, the precursor of 5$\mathrm{HT}^{445}$.

Essentially two types of mechanisms could account for a joint effect of 5-HT and NA in the action of antidepressant treatments. On one hand, these treatments could act on each neurotransmitter system independently, but simultaneously. In this case, parallel mechanisms would be involved. For instance, as described in section 8.2, TCA treatments can decrease the excitability of pyramidal neurons of the idppocampus by two parallel mechanisms, i.e. the sensitization of the inhibitory 5-HT $1 A$ receptors and the desensitization of the excitatory $\beta$-adrenoceptors. On the other hand, serial mechanisms could involve a principal action of an antidepressant treatment on one neurotransmitter system that would in turn alter the other monoaminergic system. We will focus in the next sections on serial mechanisms.

Several types of neuronal interactions can be distinguished. One type involves heteroreceptors which can be defined as presynapticreceptors activated by a neurotransmitter different from that released by the nerve terminals on which they are localized. The firstdescription of neuronal interactions through heteroreceptors was shown at the level of the cholinergic system, when it was found that NA released from the sympathetic postganglionic nerve endings tonically inhibited the release of acetylcholine from varicosities ${ }^{344}$. Evidence suggests that heteroreceptors can be activated in absence of synaptic contact. It appears, for instance, that NA released from nonsynaptic varicosities can diffuse to $\alpha 2$-adrenergic heteroreceptors localized on target cells at a greater distance than what is normally observed for conventional synaptic neurotransmission 116,455. A second way by which the NA and 5-HT systems could have "cross-talk" is via receptor-receptor interactions. In this case, adrenergic and 5-HT receptors localized on the same cell could alter each other's sensitivity or function through common transduction links at the level or: the membrane or via second messengers. Of course, these receptor-receptor interactions could occur either at the level of 5-HT or NA neurons themselves or at the level of their common postsynaptic cell targets. A third type of interaction involves direct noradrenergic or serotonergic synaptic input at the level of the soma or the dendrites of 5-HT and NA neurons. Finally, these neurons could also interact indirectly through the bias of interposed neurons, for instance through GABAergic interneurons. 


\subsection{Neurochemical evidence for interactions at presynaptic terminals}

Since the original report of Montel and Starke that clonidine inhibits the release of [3H]5-HT from preloaded cerebral cortex slices ${ }^{424}$, the existence of $\alpha 2$-adrenoceptors modulating 5-HT release has been extensively documented, in vitro, in various brain regions of several species including humans $127,139,148,158,172,174,271,357,358,386,387$. The conclusion that these $\alpha 2$-adrenergic heteroreceptors are localized on 5-HT terminals was inferred from two approaches. First, the inhibitory effect of NA on the release of $[3 \mathrm{H}] 5-\mathrm{HT}$ was found in synaptosomes, i.e. pinched-off nerve terminals from the rat brain cortex and hippocampus 294,357 . Second, the blockade of neuronal propagation of action potential with tetrodotoxin did not hinder the inhibitory effect of NA on the calcium-evoked release of [3H]5-HT from preloaded cortex slices ${ }^{172}$. However, binding studies failed to detect any change in [3H]clonidine or [3H]rauwoiscine binding after 5 -HT fiber denervation ${ }^{183}$, most likely because $\alpha_{2}$-adrenergic heteroreceptors account only for a small proportion of $\alpha 2$-adrenoceptors .

The characterization of the precise pharmacological subtype of the $\alpha 2$-adrenergic heteroreceptors has recently been done. A first study indicated that these heteroreceptors are not of the $\alpha_{2 B} B$-subtype, based in part on the fact that oxymetazoline, an $\alpha_{2 A} \mathrm{~A} / \mathrm{D}$-adrenergic agonist, acts with high potency at these sites and that prazosin, which is selective for $\alpha_{2 \mathrm{~B}}$-adrenoceptors, is not an antagonist at these sites ${ }^{165}$. It was subsequently shown that $\alpha_{2}$-adrenoceptors on 5 -HT terminals are of the $\alpha_{2 D} \mathrm{D}$ subtype in rat brain cortex, but of the $\alpha_{2 A}$ subtype in the same brain area of the rabbit ${ }^{438}$. Similarly to $\alpha_{2}$-adrenergic autoreceptors, there is some evidence that $\alpha_{2}$-adrenergic heteroreceptors on 5-HT terminals are negatively linked with adenylate cyclase. In the presence of forskolin plus an inhibitor of phosphodiesterase, the inhibitory effect of $\alpha 2$-adrenergic agonists (clonidine, B-HT 920, bromoxidine) on the release of [3H]5-HT from preloaded rat brain $ı$ slices was markedly reduced ${ }^{359,384}$. Furthermore, inactivation of $\mathrm{G}$ proteins with $\mathrm{N}$-ethylmaleimide was shown to diminish the action of clonidine at $\alpha 2$-adrenergic heteroreceptors on 5-HT terminals ${ }^{293}$. The phosphatidyl-inositol pathway could also be linked with these heteroreceptors as the activation of protein kinase $C$ with a phorbol ester was shown to shift to the right the concentration-effect curve of bromoxidine in the hypothalamus 359 .

According to the data of Blier et al..$^{59}$, there are complex receptor-receptor interactions between the $\alpha_{2}$-adrenergic heteroreceptor, the autoreceptor and the reuptake carrier on 5-HT terminals of the hypothalamus. These authors have shown that the inhibition of the electrically-evoked release of [3H]5HT by bromoxidine or NA is attenuated in the presence of SSRIs (citalopram, paroxetine). A similar finding previously indicated that the action of clonidine at $\alpha_{2}$-adrenoceptors on 5-HT terminals of the cerebral cortex is decreased in the presence of the SSRI 6-nitroquipazine ${ }^{174}$. Pretreatment with the 5HT synthesis inhibitor para-chlorophenylalanine (PCPA) was performed to determine whether the interaction between the $\alpha_{2}$-adrenergic heteroreceptors and the 5-HT reuptake carrier is due to an increased synaptic concentration of 5-HT. The inhibitions induced by NA or bromoxidine on the release of [3H]5-HT were not significantly changed by the PCPA pretreatment. However, this pretreatment markedly reduced the interaction between citalopram and bromoxidine, suggesting that the responsiveness of $\alpha 2$-adrenergic heteroreceptors is determined by the level of activation of 5-HT 1 B autoreceptors ${ }^{59}$. 
In support of this interpretation, the blockade of 5-HT1B autoreceptors with cyanopindolol was shown to prevent the inhibitory effect of citalopram on the sensitivity of the $\alpha_{2}$-adrenergic heteroreceptors 59 . Comparable receptor-receptor interactions regulating 5-HT and NA actions were also illustrated in the peripheral nervous system as mutual inhibitory actions between prejunctional $\alpha 2$-adrenoceptors and 5 $\mathrm{HT}_{1 \mathrm{~B}}$ receptors on the sympathetic nerve terminals 311.

Recent data from the group of Frazer suggest the presence of $\beta$-adrenoceptors on 5-HT terminals 207 . The selective binding of [125П]iodopindolol at $\beta_{1}$ - and $\beta_{2}$-adrenoceptors (in presence of unlabelled ligands masking either of these sites) was investigated following denervation of 5-HT terminals with 5,7-DHT. Densities of both receptors were found to be decreased in several terminal regions of the brains. The down-regulation appeared selective for the $\beta_{1}$-adrenoceptors in some areas of the hippocampus as there was a marked decrease of both $\beta_{1}$ and $\beta_{2}$ subtypes in the area $C A 3$, but only

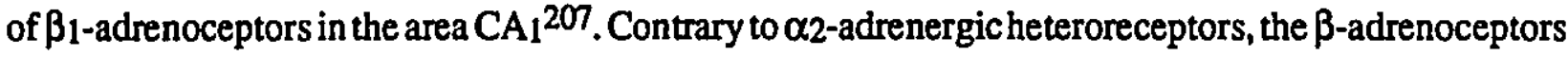
on 5-HT terminals do not appear to modulate the release of 5-HT:. First, because the inhibitory effect of NA on the evoked release of [ $3 \mathrm{H}] 5$-HT is not blocked by $\beta$-adrenergic antagonists 148,172 , and second, because $\beta$-adrenergic agonists are lacking any effect at this level $34,148,385$. Some $\beta$-adrenergic antagonists enhance the evoked release of [3H] $[\mathrm{H}-\mathrm{HT}$, but this action is thought to be related to a blockade of 5-HT 1 B autoreceptors $34,140,271,306,385$. Nevertheless, long-term treatments with the $\beta$-adrenergic agonist and putative antidepressantdrug flerobuterol was shown to increase the efficacy of the electrical stimulation of the ascending 5-HT pathway to suppress the firing activity of CA3 pyramidal neurons 68 . This effect was not due to the blockade of 5-HT1B autoreceptors as the sensitivity of these autoreceptors was found unchanged when tested by increasing the frequency of the stimulation 68 . Furthermore, the sensitivity of postsynaptic 5-HT 1 A receptors was also unaltered ${ }^{68}$. It thus appears that stimulation of $\beta$ adrenoceptors can modulate upon sustained and prolonged activation, but not acutely, the release of 5HT through some unknown mechanism.

\subsection{Neurochemical evidence for interactions at postsynaptic sites}

The " 5-HT / NA link" hypothesis of affective disorders was postulated by Sanders-Bush and Sulser 375,429 on the basis of alterations of $\beta$-adrenoceptor number and function following impairment of 5-HT neurotransmission. These suthors have reviewed evidence indicating that the binding of [3H]dihydroalprenolol is increased by 5,7-DHT lesions of 5-HT terminals as well as by pretreatments with the 5-HT synthesis inhibitor PCPA 375,429 . Furthermore, it was shown that the down-regulation of [3H]dihydroalprenolol binding normally observed following long-term treatments with various antidepressant drugs (see section 8.2.2) is not observed in animals pretreated with either 5,7-DHT or PCPA $^{375,429}$. However, subsequent studies revealed that the selective binding of [125חpindolol at $\beta$ adrenoceptors is not affected by 5,7-DHT and PCPA pretreatments 207 and that an up-regulation of postsynaptic 5-HT1B sites labelled with low affinity by [3H]dihydroalprenolol can account for the results obtained following 5,7-DHT lesions ${ }^{163,365}$. In addition, it appears that antidepressant drugs selectively down-regulate high affinity [3H]dihydroalprenolol binding and that these high affinity sites are not affected by PCPA pretreatments $\check{3} 3$. 
Nevertheless, the notion that 5 -HT regulates $\beta$-adrenocepror function cannot be readily ruled out. Denervation of 5-HT fibers with 5,7-DHT was shown to prevent the desensitization of isoproterenolstimulated adenylate cyclase by long-term desipramine treatment in the cortex ${ }^{31}$. Others, however, fail to replicate these results using 5,7-DHT or PCPA pretreatments 235,285 . Blockade of 5-HT synthesis with PCPA was also shown to increase isoproterenol-induced production of cAMP in the hippocampus 425 . Furthermore, addition of 5-HT onto hippocampal slices stimulated adenylate cyclase by itself, but did not change isoproterenol-induction of this enzyme ${ }^{316}$. In contrast, 5-HT alone did not change cAMP production in the cortex, but markedly increased isoproterenol-activation of adenylate cyclase via the activation of 5-HT2 receptors ${ }^{316}$. It thus appears that depending on the regions of the brain, 5-HT either facilitates or inhibits $\beta$-adrenergic-stimulation of cAMP.

Relatively fewer studies have been done at the level of other postsynaptic receptors. Some reports indicated that the enhancement in 5-HT neurotransmission produced by a long-term citalopram treatment increases the density of cortical, but not hippocampal, $\alpha_{1 \text {-adrenoceptors }} 283,332,452$. This effect might be linked with the capacity of citalopram to reduce the desensitization of $\alpha_{1}$-adrenoceptors by protein kinase $C^{321}$. Finally, one study 178 indicated that an intact NA system is required for the sensitization of 5-HT1A receptors by TCA in the hippocampus. Indeed, the increased efficacy of microiontophoretically-applied 5-HT to suppress the firing rate of CA3 pyramidal neurons that is induced by long-term treatments with amitriptyline was shown to be abolished by a pretreatment with 6-OHDA ${ }^{178}$.

\subsection{Behavioral evidence for interactions in the brain}

Data from several sources underscore the ability of NA to modulate 5-HT behavioral responses. These NA modulations can be separated into those that enhance 5-HT responses, most often found to be mediated by $\beta$-adrenoceptors, and those that decrease these behaviors. The earliest investigations 181,182 studied the role of the NA system on the 5-HT receptor-mediated hyperactivity. Pretreatments with the $\beta$-adrenergic antagonist (-)propranolol almost totally inhibited the hyperactivity elicited by 5 HTP $^{182}$. ECS is known to increase the hyperactivity induced by the 5-HT2 receptor agonist quipazine. Interestingly, this effect of ECS was shown to be abolished by previous lesioning of NA neurons with 6-OHDA ${ }^{181}$. Subsequent studies investigated several aspects of the so-called 5-HT syndrome: headtwitch or head-weaving, forepaw treading, hindlimb abduction. These behaviors induced by either 5 HTP or 5-HT2 agonists (quipazine or 5-methoxydimethyltryptamine) were shown to be enhanced by various $\beta$-adrenergic agonists (salbutamol, clenbuterol, terbutaline) and blocked by (-)propranolol or

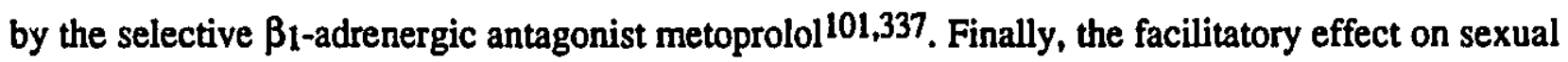
behaviors of 5-methoxydimethyltryptamine was prevented by selective lesions of NA neurons with DSP-4137.

NA appears to exert in parallel inhibitory effects on 5-HT behavioral responses through $\alpha_{2}$ adrenoceptors. Head-twitches induced by 5-methoxydimethyltryptamine or 5-HTP were potently inhibited by low doses of clonidine, and potentiated by the antagonists idazoxan and yohimbine 202 . 
These effects do not involve $\alpha_{2}$-adrenergic autoreceptors however, tut rather postsynaptic $\alpha_{2}$ adrenoceptors as 6-OHDA or DSP-4 lesions enhanced the head-twitch behavior without altering the inhibitory effect of clonidine ${ }^{202}$. NA also has antagonistic effects on 5 -HT-induced suppression of pain. Foot-shock-induced jumping in rats was shown to be decreased by different conditions that increase the stimulation of postsynaptic 5-HT receptors, such as 5-HTP, 5-methoxydimethyltryptamine or 5-HT reuptake inhibition with chlorimipramine 331 . The analgesic effect of all these drugs was abolished by pretreatments with either clonidine or with the NA releaser methamphetamine ${ }^{331}$. Direct application of 5-HT in the hippocampus dose-dependently decreased the behavioral reactivity to hot plate or footshock, but mixed application of 5-HT and NA did not have this effect ${ }^{156}$. Spinal NA depletion with 6-OHDA or DSP-4 abolished and even reversed the analgesic effects of 5-methoxydimethyltryptamine in shock titration, hot-plate and tail-flick measures of pain sensitivity 22 .

There are also behavioral data suggesting that 5 -HT may have a facilitating effect on NA terminals. Clonidine is known to produce hypoactivity or sedation via an inhibitory effect on NA neurons through the activation of $\alpha 2$-adrenergic autoreceptors ${ }^{203}$. Considering that this sedative effect of clonidine was shown to be potentiated by 5-HT denervation with 5,7-DHT 201 , it might be argued that 5-HT normally exerts an enhancing effect on NA release in some brain areas, which would counteract the inhibitory effect of clonidine at $\alpha_{2}$-adrenergic autoreceptors. It is also irteresting to note that long-term treatments with noradrenergic reuptake blockers (or MAOIs) decrease the sedative effect of clonidine 204,205,297 and that this effect was shown to be prevented by a 5,7-DHT pretreatmen ${ }^{201}$.

\subsection{Evidence for interconnections betwaen 5-HT neurons of the mesencephalic raphe nucleiand NA neurons of the locus coeruleus}

Ample evidence irdicates that the DR is the target of NA projections from the LC. Several techniques including fluorescence histochemistry, retrograde tracing with horsedish peroxidase, and elec son microscopy autoradiography have shown that 5-HT neurons in the DR are heavily innervated by $N$, p projections from the $L C 17,29,154,273,374$. Furthermore, the $D R$ area was shown to display a dense labeling with the two $\alpha_{1}$-adrenoceptor ligands [3H]WB4101 and [3H]prazosin ${ }^{443}$. The noradrenergic input was reported to be excitatory in chloral hydrate-anesthetized rats since microiontophoretic

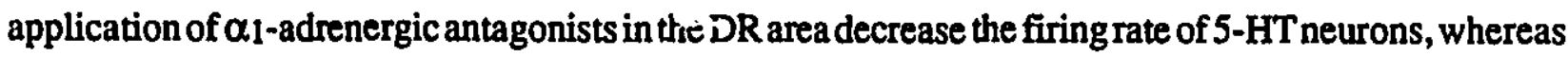

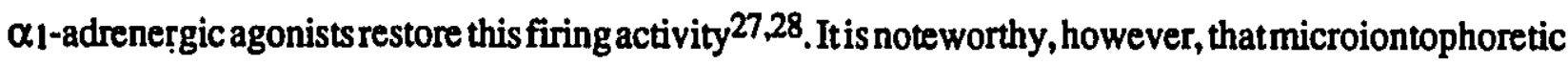
applications of NA or phenylephrine have no effect by themselves, suggesting a maximal tonic activation of $\alpha_{1}$-adrenoceptors under basal conditions 252,431 . Reserpine, which induces depletions of both 5-HT and NA, suppressed 5-HT neurons' firing ${ }^{30}$. This effect of reserpine was shown to be prevented by $6-$ OHDA lesions of $\mathrm{NA}$ terminals and reversed by amphetamine, a releaser of endogenous $\mathrm{NA}^{30}$. Agents acting at $\alpha_{2}$-adrenoceptors have the opposite effect on DR 5-HT neurons' firing activity. Indeed, intravenous administration of agonists acting at these receptors (clonidine, oxaminozoline) suppressed 5-HT neuronal discharges 119,431 , whereas the selective $\alpha_{2}$-adrenoceptor antagonists idazoxan and $(t)$ remeron enhanced by themselves the firing activity of these neurons $152,159,186$. The effect of 
clonidine is mediated by the activation of $\alpha_{2}$-adrenergic autoreceptors, which would decrease the endogenous NA excitatory input at $\alpha_{1}$-adrenoceptors on the $D R$, because it was prevented by 6-OHDA lesions of NA neurons ${ }^{431}$. However in other conditions, clonidine might also act directly at $\alpha_{2}-$ adrenoceptors, located in the somatodendritic regions of DR 5-HT neurons, to inhibit cell firing as well as the release of 5-HT150,257. Clonidine and 6-OHDA pretreatments were also shown to increase intracellular 5-HT histochemical fluorescence in the DR area, most likely through a decrease in the release of $5-\mathrm{HT}^{162}$.

The excitatory action of $\alpha_{1}$-adrenoceptors on the firing activity of DR 5-HT neurons was inferred to be physiologically important because most of the DR 5-HT neurons in brain slices obtained from rats or mice anesthetized with halothane prior to decapitation were silent $i n$ vitro unless NA or phenylephrine were added into the medium ${ }^{439,446}$. However, in brain slices from mice that were not anesthetized before decapitation, 5-HT neurons were spontaneously active and phenylephrine was inactive ${ }^{439}$. Further-

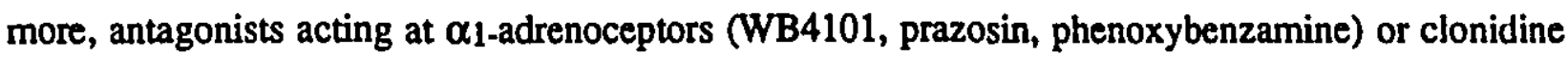
produced strong behavioral effects in freely moving cats, but did not alter DR firing activity ${ }^{209}$. It thus appears that although the excitatory NA input at DR 5-HT neurons occurs under some physiological conditions, the continuous activity of LC NA neurons is not required in order to maintain DR 5-HT neurons' firing activity232.

On the basis of some indirect data (behavioral activity and 5-HIAA concentration in terminal brain areas) it has been suggested that LC NA neurons exert an inhibitory action on the activity of MR 5-HT neurons ${ }^{124,353,354}$. Although there are NA terminals in the MR areal ${ }^{154}$ it har :.ot been shown that these projections originate from the LC. Furthermore, there is no electrophysiological indication that could substantiate the above contention.

Visualisation of $[3 \mathrm{H}] 5-\mathrm{HT}$-labeled varicosities with high-resolution autoradiography 264 , immunohistochemical localisation of tryptophan hydroxylase 351 and horseradish peroxidase (HRP) retrograde tracing 84,394 suggested the presence of 5-HT terminals at the dendritic level of LC NA neurons. However, a recent study employing a more sensitive method, tracer wheat germ agglutininconjugated HRP, did not find these serotonergic projections to the LC area 25 . Neuronal perikarya containing 5-HT have been found in the primate LC area ${ }^{408}$. The presence of 5-HT markers in the LC area might thus be explained in light of this evidence. It appears clear, nevertheless, that 5-HT exerts an inhibitory effect on NA synthesis since lesions of several raphe nuclei, including the DR and MR, increase tyrosine hydroxylase activity in the LCarea 278,299 . There is also electrophysiological evidence that 5-HT might exert a suppressant effect on the spontaneous firing activity of LCNA neurons. There is a low h": "onsistent binding to 5-HT1 sites the $\mathrm{LC}_{\mathrm{H}} \mathrm{rre}^{462}$. Although microiontophoretic application of 5-HT or 8-OHDPAT does not have any effect on LC NA neurons spontaneous activity, stimulation of presynaptic 5-HT1 receptors with 5-HT, TFMPP or 8-OHDPAT was shown to markedly decrease glutamate activation of firing activity as well as glutamatergic synaptic potentials of LC NA neurons $65,91,171$. This excitatory glutamatergic pathway connected to the LC and modulated by 5-HT most likely originates from the paragigantocellularis nucleus 25,65 . 
Similarly to 5-HT 1 ligands, local applications of 5-HT2 agonists into the LC area do not change the firing activity of NA neurons 6,171 . However, $5-\mathrm{HT}_{2}$ agonists have a prominent modulatory effect on the firing activity of LCNA neurons when administered intravenously. Indeed, mescaline, LSD and $( \pm)$-DOI have been shown to potentiate the sensory-evoked activation of LC NA neurons, and these responses were blocked by various 5-HT2 antagonists $3,6.93$. Interestingly, this response might also be mediated indirectly by the glutamatergic pathway from the paragigantocellularis nucleus since it was blocked by local application of a NMDA receptor antagonist, 2-amino-5-phosphonopentanoic acid 93 . Besides this potentiating effect on the sensory evoked activity, intravenously administered 5-HT2 agonists have also been shown to exert inhibitory effects on LC NA neurcns' spontaneous activity through postsynaptic 5-HT2 receptors $3,6,93,170,360$. The latter inhibitory effects are mediated via GABAergic fibers originating from the prepositus hypoglossal nucleus because they are blocked by local LC application of the GABA antagonist bicuculline and are prevented by electrolytic lesions of the prepositus hypoglossal nucleus 93,171 .

\section{References}

1. (1994) Diagnostic and statistical manual of mental disorders. Fourth Edition, American Psychiatric Association, Washington,DC.

2. Abercrombie, E. D., R. W. Keller, and M. J. Zigmond. (1988) Characterization of hippocampal norepinephrine release as measured by microdialysis perfusion: pharmacological and behavioral sndies. Neuroscience 27:897.

3. Aghajanian, G. K. (1980) Mescaline and LSD facilitate the activation of locus coeruleus neurons by peripheral stimuli. Brain Res. 186:492.

4. Aghajanian, G. K. and H. J. Haigler. (1974) L-Tryptophan as a selective histochemical marker for serotonergic neurons in single-cell recording studies. Brain Res. 81:364.

5. Aghajanian, G. K. and J. M. Lakoski. (1984) Hyperpolarisation of serotonergic neurons by serotonin and LSD: Studies in brain slices showing increased potassium conductance. Brain Res. 305:181.

6. Aghajanian, G. K., J. S. Sprouse, and K. Rasmussen. (1987) Physiology of the midbrain serotonin system. In Psychopharmacology: The third generation of progress. H. Y. Meltzer, editor, Raven press, New York, 141-149..

7. Aghajaniun, G. K. and Y. -Y. Wang. (1986) Pertussis toxin blocks the outward currents evoked by opiate and $\alpha_{2-}$ agonists in locus coeruleus neurons. Brain Res. 371:390.

8. Aghajanian, G. K. and Y. Y. Wang. (1987) Common $\alpha_{2}$ and opiate effector mechanisms in the locus coeruleus: intracellular studies in brain slices. Neuropharmacology 26:793.

9. Ahlquist, R. P. (1948) A study of the adrenergic receptors. Am. J. Physiol. 153:586.

10. Albert, P. R., Q. -Y. Zhou, H. H. M. Van tol, J. R. Bunzow, and O. Civelli. (1990) Cloning, functional expression, and mRNA tissue distribution of the rat 5-hydroxytryptamine ${ }_{1 \mathrm{~A}}$ receptor gene. J. Biol. Chem. 265:5825.

11. Allgaier, C., E. Agneter, T. J. Feuerstein, and E. A. Singer. (1992) Estimation of the biophase concentration of noradrenaline at presynaptic $\alpha_{2}$-adrenoceptors in brain slices. Naunyn-Schmiedeberg's Arch. Pharmacol. 345:402. 
12. Allgaier, C., T. J. Feuerstein, and G. Herting. (1986) N-ethylmaleimide (NEM) diminishes $\alpha_{2}$-adrenoceptor mediated effects on noradrenaline release. Naunyn-Schmiedeberg's Arch. Pharmacol. 333:104.

13. Allgaier, C., T. J. Feuerstein, R. Jackisch, and G. Henting. (1985) Islet-activating protein (pertussis toxin) diminishes $\alpha_{2}$-adrenoceptor mediated effects on noradrenaline release. Naunyn-Schmiedeberg's Arch. Pharmacol. 331:235.

14. Allgaier, C., H. Repp, and G. Herting. (1993) Effect of $K^{+}$channel blockers on the $\alpha_{2}$-adrenoceptor-coupled regulation of electrically evoked noradrenaline release in hippocampus. Naunyn-Schmiedeberg's Arch. Pharmacol. 347:14.

15. Alreja, M. and G. K. Aghajanian. (1991) Pacemaker activity of locus coeruleus neurons: whole cell re: ordings in brain slices show dependence on CAMP and protein kinase A. Brain Res. 556:339.

16. Amin, A. H., T. B. B. Crawford, and J. H. Gaddum. (1954) The distribution of substance P and 5 hydroxytryptamine in the central nervous system of the dog. J. Physiol. (Lond.)126:596.

17. Anderson, C., D. Pasquier, W. Forbes, and P. Morgane. (1977) Locus coeruleus-to-dorsal raphe input examined by electrophysiological and morphological methods. Brain Res. Bull. 2:209.

18. Andrade, R. (1992) Electrophysiology of 5-HTiA receptors in the rat hippocampus and cortex. Drug Dev. Res. 26:275.

19. Andrade, R. and G. K. Aghajanian. (1985) Opiate- and $\alpha_{2}$-adrenoceptor-induced hyperpolarizations of locus ceruleus neurons in brain slices: reversal by cyclic adenosine 3':5'-monophosphate analogues. J. Neurosci. 5:2359.

20. Andrade, R. and Y. Chaput. (1991) 5-HT 4 like receptors mediate the slow excitatory response to serotonin in the rat hippocamipus. J. Pharmacol. Exp. Ther. 257:930.

21. Andrade, R. and R. A. Nicoll. (1987) Pharmacologically distinct actions of serotonin on single pyramidal neurones of the rat hippocampus recorded in vitro. J. Physiol. (Lond) 394:99.

22. Archer, T., G. Jonsson, B. G. Minor, and C. Post. (1986) Noradrenergic-serotonergic interactions and nociception in the rat. Eur. J. Pharmacol. 120:295.

23. Asnis, G. M., S. Wetzler, W. C. Sanderson, R. S. Kahn, and H. M. van Praag. (1992) Functional interrelationship of serotonin and norepinephrine: Cortisol response to $\mathrm{mCPP}$ and DMI in patients with panic disorder, patients with depression, and normal control subjects. Psychiat. Res. 43:65.

24. Aston-Jones, G. and F. E. Bloom. (1981) Activity of NA containing locus coeruleus neurons in behaving rats anticipates fluctuations in the sleep waking cycle. J. Neurosci. 1:876.

25. Aston-Jones, G., M. Ennis, W. A. Pieribone, W. T. Nickell, and M. T. Shipley. (1986) The brain nucleus locus coeruleus: restricted afferent control of a broad efferent network. Science 234:734.

26. Azmitia, E. C. and M. Segal. (1978) An autoradiographic analysis of the differential ascending projections of the dorsal and median raphe nuclei in the rat. J. Comp. Neurol. 179:641.

27. Baraban, J. M. and G. K. Aghajanian. (1980) Suppression of serotonergic neuronal firing by $\alpha$-adrenoceptor antagonists: evidence against GABA mediation. Eur. J. Pharmacol. 66:287.

28. Baraban. J. M. and G. K. Aghajanian. (1980) Suppression of firing activity of 5-HT neurons in the dorsal raphe by alpha-adrenoceptor antagonists. Neuropharmacology 19:355.

29. Baraban, J. M. and G. K. Aghajanian. (1981) Noradrenergic innervation of serolonergic neurons in the dorsal raphe: Demonstration by electron microscopic autoradiography. Brain Res. 204:1. 
30. Baraban, J. M., R. Y. Wang, and G. K. Aghajanian. (1978) Reserpine suppression of dorsal raphe neuronal firing: mediation by adrenergic system. Eur. J. Pharmacol. 52:27.

31. Barbaccia, M. L., N. Brunello, D. M. Chuang, and E. Costa. (1983) On the nlode of action of imipramine: Relationship between serotonergic axon terminal function and down-regulation o: $\beta$-adrenergic receptors. Neuropharmacology 22:373.

32. Barturen, F. and J. A. Garcia-Sevilla. (1992) Long term treatment with desipramine increases the turnover of $\alpha_{2-}$ adrenoceptors in the rat brain. Mol. Pharmacol. $42: 846$.

33. Bauer, M.E. and S. M. Tejani-Butt. (1992) Effects of repeated administration of desipramine or electroconvulsive shock on norepinephrine uptake sites measured by $\left[{ }^{3} \mathrm{H}\right]$ nisoxetine autoradiography. Brain Res. 582:208.

34. Baumann, P. A. and P. C. Waldmeier. (1981) Further evidence for negative feedback control of serotonin release in the central nervous system. Naunyn-Schmiedeberg's Arch. Pharmacol. 317:36.

35. Beck, A. T. (1967) Depression: Clinical, experimental and theoritical aspects. Harper \& Row, New York.

36. Beck, S. G. (1992) 5-Hydroxytryptamine increases excitability of $C A_{1}$ hippocampal pyramidal cells. Synapse 10:334.

37. Beck, S. G., W. P. Clarke, and J. Goldfard. (1985) Spiperone differentiates multiple 5-hydroxytryptamine responses in rat hippocampal slices in vitro. Eur. J. Pharmacol. 116:195.

38. Beck. S. G. and J. Goldfarb. (1985) Serotonin produces a reversible concentration dependent decrease of population spikes in rat hippocampal slices. Life Sci. 36:557.

39. Beck, S. G. and P. M. Halloran. (1989) Imipramine alters $\beta$-adrenergic, but not serotonergic mediated responses in rat hippocampal pyramidal cells. Brain Res. 504:72.

40. Bijak, M. (1989) Antidepressant drugs potentiate the $\alpha_{l}$-adrenoceptor effect in hippocampal slices. Eur.J. Pharmacol. 166:183.

41. Blier, P. (1991) Teminal serotonin autoreceptor function in the rat hippocampus is not modified by pertussis and cholera toxins. Naunyn-Schmiedeberg's Arch. Pharmacol. 344:160.

42. Blier, P. and C. Boucnard. (1992) Effect of repeated electroconvulsive shocks on serotonergic neurons. Eur. J. Pharmacol. 211:365.

43. Blier, P. and C. Bouchard. (1993) Functional characterization of a 5-HT3 receptor which modulates the release of 5 !?T in the guinea-pig brain. Br. J. Pharmacol. 108:13.

44. Blier, P. and C. Bouchard. (1994) Modulation of 5-HT release in the guinea pig brain following long-term administration of antidepressant drugs. Br.J. Pharmacol. (in press)

45. Blier, P., Y. Chaput, and C. de Montigny. (1988) Long-term 5-HT reuptake blockade, but not monoamine oxidase inhibirion, decreases the function of terminal 5-HT autoreceptors: an electrophysiological study in the rat brain. Naunyn-Schmiedeberg's Arch. Pharmacol. 337:246.

46. Blier, P., O. Curet, Y. Chaput, and C. de Montigny. (1991) Tandospirone and its metabolite, 1-(2-pyrimidinyl)piperazine-II. Effects of acute administration of 1-PP and long-term administration of tandospirone on noradrenergic neurotransmission. Neuropharmacology 7:691.

47. Blier, P. and C. de Montigny. (1980) Effect of chronic tricyclic antidepressant treatment on the serotonergic autoreceptor: a microiontophoretic study in the rat. Naunyn-Schmiedeberg's Arch. Pharmacol. 314:123. 
48. Blier, P. and C. de Montigny. (1983) Electrophysiological investigations on the effect of repented zimelidine administration on serotonergic neurotransmission in the rat. J. Neurosci. 3:1270.

49. Blier, P. and C. de Montigny. (1985) Short-term lithium administration enhances serotonergic neurotransmission: Electrophysiological evidence in the rat CNS. Eur. J. Pharmacol. 113:69.

50. Blier, P. and C. de Montigny. (1985) Serotonergic but not noradrenergic neurons in rat central nervous system adapt to long-term treatment with monoamine oxidase inhibitors. Neuroscience 16:949.

51. Blier, P. and C. de Montigny. (1987) Modification of 5-HT neuron properties by sustained administration of the 5-HT $1 \mathrm{~A}$ agonist gepirone: electrophysiological studies in the rat brain. Synapse 1:470.

52. Blier, P. and C. de Montigny. (1987) Antidepressant monoamine oxidase inhibitors enhance serotonin but not norepinephrine neurotransmission. Psychopharmacol. Series 3:127.

53. Blier, P. and C. de Montigny. (1990) Differential effect of gepirone on presynaptic and postsynaptic serotonin receptors: single-cell recording studies. J. Clin. Psychopharmacol. 10:13S.

54. Blier, P., C. de Montigny, and A. Azzaro. (1986) Modification of serotonergic and noradrenergic neurotransmissions by repeated administration of monoamine oxidase inhibitors: Electrophysiological studies in the rat central nervous system. J. Pharmacol. Exp. Ther. 237:987.

55. Blier, P., C. de Montigny, and A. Azzaro. (1986) Effect of repeated amiflamine administration on serotonergic and noradrenergic neurotransmission: Electrophysiological studies in the rat CNS. Naunyn-Schmiedeberg's Arch. Pharmacol. 334:253.

56. Blier, P., C. de Montigny, and Y. Chaput. (1987) Modifications of the serotonin system by antidepressant trentments: implications for the therapeutic response in major depression. J. Clin. Psychopharmacol. 7:24S.

57. Blier, P., C. de Montigny, and Y. Chaput. (1990) A role for the serotonin system in the mechanism of action of antidepressant treatments: preclinical evidence.J. Clin. Psychiatry. 51 Suppl:14.

58. Blier, P., C. de Montigny, and D. Tardif. (1984) Effects of the two antidepressant drugs mianserin and indalpine on the serotonergic system: Single-cell studies in the rat. Psychopharmacology 84:242.

59. Blier, P., A. M. Galzin, and S. Z. Langer. (1990) Interaction between serotonin uptake inhibitors and alpha-2 adrenergic heteroreceptors in the rat hypothalamus. J. Pharmacol. Exp. Ther. 254:236.

60. Blier, P., A. Lista, and C. de Montigny. (1993) Differential properties of pre- and postsynaptic 5hydroxytrypttamineit receptors in the dorsal raphe and hippocampus: il. Effect of pertussis and cholera toxins. $J$. Pharmacol. Exp. Ther. 265:16.

61. Blier, P., A. Lista, and C. de Montigny. (1993) Differential properties of pre- and postsynaptic 5hydrox ytryptamine 1 a receptors in the dorsal raphe and hippocampus: effect of spiperone. J. Pharmacol. Exp. 'Ther. 265:7.

62. Blier, P., P. J. Monroe, C. Bouchard, D. L. Smith, and D. J. Smith. (1993) 5-HT3 receptors which modulate [3H]5-HT release in the guinea pig hypothalamus are not autoreceptors. Synapse 15:143.

63. Blier, P., A. Serrano, and B. Scatton. (1990) Differential responsiveness of the rat dorsal and median raphe 5-HT systems to 5-HT 1 receptor agonists and p-chloroamphetamine. Synapse 5:120.

64. Blier, P., S. Steinberg, Y. Chaput, and C. de Montigny. (1989) Electrophysiological assessment of putative antagonists of 5-hydroxytryptamine receptors: a single-cell study in the rat dorsal raphe nucleus. Can.J. Physiol. Pharmacol. 67:98. 
65. Bobker, D. H. and J. T. Williams. (1989) Serotonin agonists inhibit synaptic potentials in the rat locus coeruleus in vitro via 5-HT $1 \mathrm{~A}$ and 5-HT, $1 \mathrm{~B}$ receptors. J. Pharmacol. Exp. Ther. 250:37.

66. Boess, F. G. and I. L. Martin. (1994) Molecular biology of 5-HT receptors. Neuropharmacology 33:275.

67. Bousquet, P., G. Bricca, M. Dontenwill, J. Feldman, H. Greney, A. Belcourt, J. Stutzmann, and E. Tibiriça. (1992) From the $\alpha_{2}$-adrenoceptors to the imidazoline preferring receptors. Fundam. Clin. Pharmacol. 6 Suppl. 1:15s.

68. Bouthillier, A., P. Blier, and C. de Montigny. (1991) Flerobuterol, a $\beta$-adrenoceptor agonist, enhances serotonergic neurotransmission: an electrophysiological study in the rat brain. Psychopharmacology 103:357.

69. Bowyer, J. F. and N. Weiner. (1990) $\alpha_{2}$-Adrenergic inhibition of calcium-evoked $3 \mathrm{H}$-norepinephrine release from synaptosomes is blocked by depolarization. J. Pharmacol. Exp. Ther. 253:1063.

70. Bradley, P. B., G. Engel, W. Feniuk, J. R. Fozard, P. P. Humphrey, D. N. Middlemiss, E. J. Mylecharane, B. P. Richardson, and P. R. Saxena. (1986) Proposals for the classification and nomenclature of functional receptors for 5hydroxytryptamine. Neuropharmarnlogy 25:563.

71. Brady, L. S., P. W. Gold, M. Herkenham, A. B. Lynn, and H. J. Whitfield,Jr.. (1992) The antidepressants fluoxetine, idazoxan and phenelzine alter corticotropin-releasing hormone and tyrosine hydroxylase mRNA levels in rat brain: therapeutic implications. Brain Res. 572:117.

72. Brady, L. S., H. J. Whitfield,Jr., R. J. Fox, P. W. Gold, and M. Herkenham. (1991) Long-term antidepressant administration alters corticotropin-releasing homone, tyrosine hydroxylase, and mineralocorticoid receptor gene expression in rat brain. Therapeutic implications. J. Clin. Invest. 87:831.

73. Bricca, G., M. Dontenwill, A. Molines, J. Feldman, A. Belcourt, and P. Bousquet. (1988) The imidazoline preferring receptor: binding studies in bovine, rat and human brainstem. Eur. J. Pharmacol. 150:401.

74. Broderick, P. A. and M. F. Piercey. (1991) 5-HT 1 A agonists uncouple noradrenergic somatodendritic impulse flow and terminal release. Brain Res. Bull. 27:693.

75. Brodie, B. B. and P. A. Shore. (1957) A concept for a role of serotonin and norepinephrine as chemical mediators in the brain. Ann. N. Y. Acad. Sci. 66:631.

76. Brown, T. H. and A. M. Zador. (1990) Hippocampus. In The synaptic organization of the brain. G. M. Shepherd, editor. Oxford University Press, New York. 346-388.

77. Brunello, N., M. L. Barbaccia, D. M. Chuang, and E. Costa. (1982) Down-regulation of $\beta$-adrenergic receptors following repeated injections of desmethylimipramine: permissive role of serotonergic axons. Neuropharmacology 21:1145.

78. Burgard, E. C., G. Decker, and J. M. Sarvey. (1989) NMDA receptor antagonists block norepinephrine-induced long-lasting potentiation and long-term potentiation in rat dentate gyrus. Brain Res. 482:351.

79. Bumet, P. W. J., D. Michelson, M. A. Smith, P. W. Gold, and E. M. Stemberg. (1994) The effect of chronic imipramine administration on the densities of 5-HT $1 \mathrm{~A}$ and 5-HT 2 receptors and the abundancies of 5-HT receptor and transporter mRNA in the cortex, hippocampus and dorsal raphe of three strains of rat. Brain Res. 638:311.

80. Cage, F. H., A. Bjorklund, and U. Stenevi. (1983) Reinnervation of the partially deafferented hippocampus by compensatory collateral sprouting from spared cholinergic and noradrenergic afferents. Brain Res. 268:27.

81. Campbell, 1. C. and R. M. McKeman. (1986) Clorgyline and desipramine alter the sensitivity of [3H]noradrenaline release to calcium but not to clonidine. Brain Res. 372:253.

82. Cannon, W. B. and J. E. Uridil. (1921) Studies on the conditions of activity in endocrine glands. VIII. Some effects on the denervated heart of stimulating the nerves of the liver. Am.J.P. yysiol. 58:353. 
83. Cedarbaum, J. M. and G. K. Aghajanian. (1976) Noradrenergic neurons of the lucus coeruleus: Inhibition by epinephrine and activation by $\alpha$-antagonist piperoxane. Brain Res. 112:413.

84. Cedarbaum. J. M. and G. K. Aghajanian. (1978) Afferent projections to the rat locus coeruleus as determined by a retrograde technique. J. Comp. Neurol. 178:1.

85. Chaput, Y., R. C. Araneda, and R. Andrade. (1990) Pharmacological and functional analysis of a novel serotonin receptor in the rat hippocampus. Eur.J. Pharmacol. 182:441.

86. Chaput, Y., P. Blier, and C. de Montigny. (1986) In vivo electrophysiological evidence for the regulatory role of autoreceptors on serotonergic terminals. J. Neurosci. 6:2796.

87. Chaput, Y. and C. de Montigny. (1988) Effects of the 5-HT 1 receptor antagonist, BMY7378, on 5-HT neurotransmission: Electrophysiological studies in the rat central nervous system. J. Pharmacol. Exp. Ther. 246:359.

88. Chaput, Y. and C. de Montigny. (1988) Effects of the 5-hydroxytryptamine receptor antagonist, BMY 7378, on 5hydroxytryptamine neurotransmission: Electrophysiological studies in the rat central nervous system. J. Pharmacol. Exp. Ther. 246:359.

89. Chaput, Y., C. de Montigny, and P. Blier. (1986) Effects of a selective 5-HT reuptake blocker, citalopram, on the sensitivity of 5-HT autoreceptors: electrophysiological studies in the rat brain. Naunyn-Schmiedeberg's Arch. Pharmacol. 333:342.

90. Chaput, Y., C. de Montigny, and P. Blier. (1991) Presynaptic and postsynaptic molifications of the serotonin system by long-term administration of antidepressant treatments: An in vivo electrophysiologic study in the rat. Neuropsychopharmacology. 5:219.

91. Charlety, P. J., G. Aston-Jones, H. Akaoka, M. Buda, and G. Chouvet. (1991) 5-HT decreases glutumate-evoked activation of locus coeruleus neurons through 5-HTIA receptors. Comp. Rend. Acad. Sci. 312:421.

92. Cheetham, S. C., M. R. Crompton, C. L. E. Katona, and R. W. Horton. (1990) Brain 5-HT, binding sites in depressed suicides. Psychopharmacology 102:544.

93. Chiang, C. and G. Aston-Jones. (1993) A 5-hydroxytryptaminez agonist augments $\gamma$-aminobutyric acid and excitatory amino acid inputs to noradrenergic locus coeruleus neurons. Neuroscience 54:409.

94. Clarke, W. P., M. De Vivo, S. G. Beck, S. Maayani, and J. Goldfarb. (1987) Serotonin decreases population spike amplitude in hippocampal cells through a pertussis toxin substrate. Brain Res. 410:357.

95. Cohen, R. M., R. P. Ebsstein, J. W. Daly, and D. L. Murphy. (1982) Chronic effects of a monoamine oxidase inhibiting antidepressant: decrease in functional alpha-adrenergic autoreceptors precede the decrease in norepinephrine-stimulated cyclic adenosine 3':5'-monophosphate systems in rat brain. J. Neurosci. 2:1588.

96. Colino, A. and J. V. Halliwell. (1986) 8-OH-DPAT is a strong antagonist of 5-HT action in rat hippocampus. Eur. J. Pharmacol. 130:151.

97. Colino, A. and J. V. Halliwell. (1987) Differential modulation of three separate potassium-conductances in hippocampal $C A_{1}$ neurons by serotonin. Nature 328:73.

98. Conrad, L. C. A., C. M. Leonard, and D. W. Pfaff. (1974) Connections of the median and dorsal raphe nuclei in the rat: An autoradiographic and degeneration study. J. Comp. Neurol. 156:179.

99. Cooper, J. R., F. E. Bloom, and R. H. Roth. (1991) The biochemical basis of neuropharmacology. Oxford, New York. 220.379.

:0. Corradetti, R., L. Ballerini, A. M. Pugliese, and G. Pepeu. (1992) Serotonin blocks the long-term potentiation induced by primed burst stimulation in the $C A_{1}$ region of rat hipposampal slices. Neuroscience $46: 511$. 
101. Cowen, P. J., D. G. Grahame-Smith, A. R. Green, and D. J. Heal. (1982) B-Adrenoceptor agonists enhance 5hydroxytryptamine-mediated behavioral responses. Br. J. Pharmacol. 76:265.

102. Curet, O. and C. de Montigny. (1988) Electrophysiological characterization of adrenoceptors in the rat dorsal hippocampus. II. Receptors mediating the effect of synaptically released norepinephrine. Brain Res. 475:47.

103. Curet, O. and C. de Montigny. (1988) Electrophysiological characterization of adrenoceptors in the rat dorsal hippocampus. I. Receptors mediating the effect of microiontophoretically applied norepinephrine. Brain Res. 475:35.

104. Curet, O. and C. de Montigny. (1989) Electrophysiological characterization of adrenoceptors in the rat dorsal hippocampus. III. Evidence for the physiological role of terminal $\alpha_{2}$-adrenergic autoreceptors. Brain Res. 499:18.

105. Curet, O., C. de Montigny, and P. Blier. (1992) Effect of desipramine and amphetamine on noradrenergic neurotransmission: electrophysiological studies in the rat brain. Eur. J. Pharmacol.

106. Curet, O.. T. Dennis, and B. Scatton. (1987) Evidence for the involvement of presynaptic $\alpha_{2}$-adrenoceptors in the regulation of norepinephrine metabolism in the rat brain. J. Pharmacol. Exp. Ther. 240:327.

107. Curtis, A. L. and R. J. Valentino. (1991) Acute and chronic effects of the atypical antidepressant, mianserin on brain noradrenergic neurons. Psychopharmacology 103:330.

108. Dahl, D. and J. M. Sarvey. (1989) Norepinephrine induces pathway-specific long-lasting potentiation and depression in the hippocampal dentate gyrus. Proc. Natl. Acad. Sci. U. S A. 86:4776.

109. Dahl, D. and J. Winson. (1985) Action of norepinephrine in the dentate gyrus. I. Stimulation of locus coeruleus. Exp. Brain Res. 59:491.

110. de Montigny, C. (1984) Electroconvulsive shock treatments enhance responsiveness of forebrain neurons to serotonin. J. Pharmacol. Exp. Ther. 228:230.

111. de Montigny, C. and G. K. Aghajanian. (1978) Tricyclic antidepressants: Long-term treatment increases responsivity of rat forebrain neurons to serotonin. Science 202:1303.

112. de Montigny, C., P. Blier, G. Caille, and E. Kouassi. (1981) Pre- and postsynaptic effect of zimelidine and norzimelidine on the scrotonergic system: Single cells study in the rat. Acta Psychiatr. Scand. 63 suppl. 290:79.

113. De Vivo, M. and S. Maayani. (1986) Characterization of the S-hydroxytryptamine1A receptor-mediated inhibition of forskolin-stimulated adenylate cyclase activity in guinea pig and rat hippocampal membranes. J. Pharmacol. Exp. Ther. 238:248.

114. Deakin, J. F., F. G. Craeff, and F. Guimaraes. (1992) 5-HT receptor subtypes and the modulation of aversion. In Central serotonin receptors and psychotropic drugs. C. . Marsden and D. J. Heal, editors. Blackwell, London. 147174.

115. Deakin, J. F. W. (1989) 5-HT reccptor subtypes in depression. In Behavioural pharmacology of 5-HT. P. Beram, A. R. Cools, and T. Archer, editors. Hillsdale, New Jersey. 179-204.

116. Descarries, L., K. D. Watkins, and Y. Lapierre. (1977) Noradrenergic axon terminals in the cerebral cortex of rat. III. Topometric ultrastructural analysis. Brain Res. 133:197.

117. Dijcks, F. A., G. S. Ruigt, and J. S. De Graaf. (1991) Antidepressants affect amine modulation of neurotransmission in the rat hippocampal slice-I. Delayed effects. Neuropharmacology 30:1141.

118. Doze, V. A., G. A. Cohen, and D. V. Madison. (1991) Synaptic localization of adrenergic disinhibition in the rat hippocampus. Neuron 6:889. 
119. Dresse, A. and J. Scuvee Moreau. (1986) Influence of the $\alpha_{2}$ agonist oxaminozoline (S3341) on firing rate of central noradrenergic and serotonergic neurons in the rat. Comparison with clonidine. Arch. Int. Physiol. Biochim. 94:99.

120. Dumuis, A., R. Bouhelal, M. Sebben, and J. Bockaert. (1988) A S-HT receptor in the central nervous system, positively coupled with adenylate cyclase, is antagonized by ICS 205930. Eur. J. Pharmacol. 146: 187.

121. Dumuis, A., R. Bouhelal, M. Sebben, R. Cory, and J. Bockaert. (1988) A nonclassical 5-hydroxytryptamine receptor positively coupled with adenylate cyclase in the central nervous system. Mol. Pharmacol. 34:880.

122. Dumuis, A., M. Sebben, and J. Bockaert. (1988) Pharmacology of 5-hydroxytryptamine-1A receptors which inhibit cAMP production in hippocampal and cortical neurons in primary culture. Mol. Pharmacol. 33:178.

123. Duncan, G. E., I. A. Paul, K. R. Powell, J. B. Fassberg, W. E. Stumpf, and G. R. Breese. (1989)

Neuroanatomically selective down-regulation of beta adrenergic receptors by chronic imipramine treatment: relationships to the topography of [3H]imipramine and [3H] desipramine binding sites.J. Pharmacol. Exp. Ther. 248:470.

124. Dyr, W., W. Kostowski, B. Zacharski, and A. Bidzinski. (1983) differential clonidine effects on EEG following lesions of the dorsal and median raphe nuclei in rats. Pharmacol. Biochem. Behav. 19:177.

125. Ebert, M. H., R. J. Baldessarini, L. F. Lipinski, and K. Berv. (1973) Effects of electroconvulsive seizures on amine metabolism in the rat brain. Arch. Gen. Psychiatry 29:397.

126. Eichenbaum, H. and T. Otto. (1993) LTP and memory: can we enhance the connection? Trends Neurosci. 16:163.

127. Ellison, D. W. and I. C. Campbell. (1986) Studies on the role of $\alpha_{2}$-adrenoceptors in the control of synaptosomal 3H-5-hydroxytryptamine release: effects of antidepressant drugs. J. Neurochem. 46:218.

128. Elmes, D. G., L. E. Jarrard, and P. D. Swart. (1975) Helplessness in hippocampectomised rats: response perseveration? Physiol. Psychol. 3:51.

129. Engberg, G. (1989) A metabollite of buspirone increases locus coeruleus activity via $\alpha_{2}$-receptor blockage. $J$. Neural Transm. 76:91.

130. Engberg, G. and E. Eriksson. (1991) Effects of $\alpha_{2}$-adrenoceptor agonists on locus coeruleus firing rate and brain noradrenaline turnover in N-ethoxycarbonyl-2ethoxy-1,2-dihydroquinoline (EEDQ)-treated rats. NaunynSchiniedeberg's Arch. Pharmacol. 343:472.

131. Engel, G., M. Gothert, D. Hoyer, E. Schlicker, and K. Hillenbrand. (1986) Identity of inhibitory presynaptic 5hydroxytryptamine (5-HT) autoreceptors in the rat brain cortex with 5-HT 1 B binding sites. Naunyn-Schmiedeberg's Arch. Pharmacol. 332:1.

132. Engel, J., L. C. F. Hanson, and B. -E. Roos. (1971) Effect of electroshock on 5-HT metabolism in rat brain. Psychopharmacology 20:197.

133. Emsberger, P. R., M. P. Meeley, J. J. Mann, and D. J. Reis. (1987) Clonidine binds to imidazole binding sites as well as $\alpha_{2}$-adrenoceptors in the ventrolateral medulla. Eur. J. Pharmacol. 134:1.

134. Ernsberger, P. R., K. L. Westbrooks, M. O. Christen, and S. G. Schafer. (1992) A second generation of centrally acting antihypertensive agents act on putative $\mathrm{I}_{1}$-imidazoline receptors. J. Cardiovasc. Pharmacol. 20:\$1.

135. Euler, U. S. (1954) Adrenaline and noradrenaline. Distribution and action. Pharmacol. Rev, 6:15.

136. Fanelli, R. J. and K. McMonagle-Strucko. (1992) Alteration of 5-HT 1 A receptor binding sites following chronic treatment with ipsapirone measured by quantitative autoradiography. Synapse 12:75. 
137. Fernandez-Guasti, A., S. Hansen, T. Archer, and G. Jonsson. (1985) Noradrenaline-serotonin interaction in the control of sexual behavior in the male rat: DSP4-induced noradrenaline depletion antagonizes the facilitory effect of serotonin receptor agonists. Brain Res. 377:112.

138. Feuerstein, T. J., C. Allgaier, and G. Henting. (1987) Possible involvement of protein kinase C (PKC) in the regulation of electrically evoked serotonin (5-HT) release from rabbit hippocampal slices. Eur. J. Pharmacol. 139:267.

139. Feuerstein, T. J., G. Hertting, and R. Jackisch. (1985) Endogenous noradrenaline as modulator of hippocampal 5HT release. Naunyn-Schmiedeberg's Arch. Pharmacol. 329:216.

140. Feuerstein, T. J., A. Lupp, and G. Herting. (1987) The serotonin (5-HT) autoreceptor in the hippocampus of the rabbit: role of 5-HT biophase concentration. Neuropharmacology 26:1071.

141. Feuerstein, T. J., E. Neuschwander, W. Sauermann, and A. Lupp. (1991) The conditions of calcium entry via Ltype channels for induction of serotonin release from rabbit hippocampus. Eur. J. Pharmacol. 198:37.

142. Finberg, J. P. M., G. Ari, G. Lavian, and D. Hovevey-Sion. (1990) Modification of $\alpha_{2}$-presynaptic receptor activity and catecholamine release following chronic MAO inhibition. J. Neural Transm. suppl. 32:405.

143. Finberg, J. P. M. and A. Tal. (1985) Reduced peripheral presynaptic adrenoceptor sensitivity following chronic antidepressant treatment in rats. Br. J. Pharmacol. 84:609.

144. Finberg, J. P. M. and M. B. H. Youdim. (1983) Selective MAO A and B inhibitors: Their mechanism of action and pharmacology. Neuropharmacology 22:441.

145. Fischette, C. T., B. Nock, and K. Renner. (1987) Effects of 5,7-dihydroxytryptamine on serotonin 1 and serotonin2 receptors throughout the rat central nervous system using quantitative autoradiography. Brain Res. 421:263.

146. Fowler, C. J., W. Danysz, and T. Archer. (1986) Noradrenaline-stimulated inositol phospholipid breakdown as a

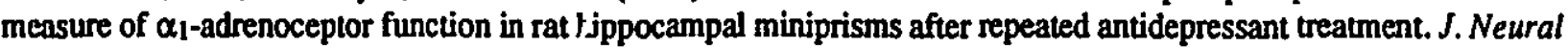
Transm. 66:197.

147. Frances, A., D. Manning, D. Marin, J. Kocsis, K. McKinney, W. Hall, and M. Kline. (1992) Relationship of anxiety and depression. Psychopharmacology 106 Suppl.:S82.

148. Frankhuijzen, A. L. and A. H. Mulder. (1980) Noradrenaline inhibits depolarization-induced 3H-5-HT release from slices of rat hippocampus. Eur. J. Pharmacol. 63:179.

149. Frankhuijzen, A. L. and A. H. Mulder. (1982) Phamacological characterization of presynaptic alphaadrenoceptors modulating 3H-NA and 3H-5-HT release form slices of the hippocampus of the rat. Eur. J. Pharmacol. 81:97.

150. Frankhuijzen, A. L., G. Wardeh, F. Hogenboom, and A. H. Mulder. (1988) $\alpha_{2}$-Adrenoceptor mediated inhibition of the release of radiolabelled 5-hydroxytryptamine and noradrenaline from slices of the dorsal region of the rat brain. Naunyn-Schmiedeberg's Arch. Pharmacol. 337:255.

151. F.azer, A. and J. G. Hensler. (1994) Serotonin. In Basic Neurochemistry: Molecular, cellular, and medical aspects. G. J. Siegel, B. W. Agranoff, R. W. Albers, and P. B. Molinoff, editors. Raven, New York. 283-308.

152. Freedman, J. E. and G. K. Aghajanian. (1984) Idazoxan selectively antagonizes $\alpha_{2}$-adrenoceptors on rat central neurons. Eur. J. Pharmacol. 105:265.

153. Freund, T. F., A. I. Gulyas, L. Acsady, T. Gorcs, and X. Toth. (1990) Serotonergic control of the hippocampus via local inhibitory intemetrons. Proc. Natl. Acad. Sci. U. S A. 87:8501.

154. Fuxe, K. (1965) The distribution of monoamine terminals in the central nervous system. Acta Physiol. Scand. 64 (Suppl. 247):37. 
155. Gaddum, J. H. (1953) Antagonism between LSD and 5-hydroxytryptamine. J. Physiol. (Lond.) 121:15p.

156. Gage, F. H. and J. E. Springer. (1981) Behavioral assessment of norepinephrine and serotonin function and interaction in the hippocampal formation. Pharmacol. Biochem. Behav. 14:815.

157. Gallager, D. W. and W. E. Bunney. (1979) Failure of chronic lithium treatment to block tricyclic antidepressantinduced 5-HT supersensitivity. Naunyn-Schmiedeberg's Arch. Pharmacol. 307:129.

158. Galzin, A. M., M. F. Poirier, A. Lista, J. P. Chodkiewicz, P. Blier, R. Ramdine, Loo H,, F. X. Roux, A. Redondo. and S. Z. Langer. (1992) Characterization of the 5-hydroxytryptamine receptor modulating the release of [3H]5hydroxytryptamine in slices of the human neocortex. J. Neurochem. 59:1293.

159. Garratt, J. C., F. Crespi, R. Mason, and C. A. Marsden. (1991) Effects of idazoxan on dorsal raphe 5hydroxytryptamine neuronal function. Eur. J. Pharmacol. 193:87.

160. Gerald, C., N. Adham, H. T. Kao, L. E. Schechter, M. A. Olsen, J. A. Bard, T. M. Laz, P. J. J. Vaysse, T. A. Branchek, and R. L. Weinshank. (1994) The 5-HT 4 receptor: Molecular cloning of two splice variants. IUPHAR satellite meeting on serotonin 3:54.

161. Gershon, E. S., Berrettini, W., Numberger, J. Jr., Goldin L.R. (1987) The search for genetic markers in affective disorders. In Psychopharmacology: The third generation of progress. H. Y. Meltzer, editor. Raven press, New York. $481-492$.

162. Geyer, M. A. and E. H. Lee. (1984) Effects of clonidine, piperoxane and locus coeruleus lesion on the serotonergic and dopaminergic systems in raphe and caudate nucleus. Biochem. Pharmacol. 33:3399.

163. Gillespie, D. D., D. H. Manier, E. Sanders-Bush, and F. Sulser. (1988) The serotonin/norepinephrine-link in brain. II Role of serotonin in the regulation of $\beta$-adrenoceptors in the low agonist affinity conformation. J. Pharmacol. Exp. Ther. 244:154.

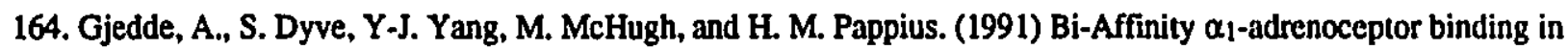
normal rat brain in vivo. Synapse 9:1.

165. Gobbi, M., E. Frittoli, and T. Mennini. (1990) The modulation of 3H-noradrenaline and 3H-serotonin releasc from rat brain synaptosomes is not mediated by the $\alpha_{2 B}$-adrenoceptor subtype. Naunyn-Schmiedeberg's Arch. Pharmacol. 342:382.

166. Godbout, R., Y. Chaput, P. Blier, and C. de Montigny. (1991) Tandospirone and its metabolite, 1-(2pyrimidinyl)-piperazine-I. Effects of acute and long-term administration of tandospirone on serotonin neurotransmision. Neuropharmacology 7:679.

167. Goldfard, J. (1990) Electrophysiologic studies of serotonin receptor activation. Neuropsychopharmacology 3:435.

168. Goldstein, J. M., L. C. Knobloch, and J. B. Malick. (1983) Electrophysiological demonstration of bolh $\alpha_{2}-$ agonist and antagonist properties of RX 781094. Eur. J. Pharmacol. 91:101.

169. Goodman Gilman, A., L. S. Goodman, and A. Gilman. (1980) The pharmacological basis of therapeutics. McMillan, New York.

170. Gorea, E. and J. Adrien. (1988) Serotonergic regulation of noradrenergic coerulean neurons: electrophysiological evidence for implication of 5-HT2 receptors. Eur. J. Pharmacol. 154:285.

171. Gorea, E., D. Davenne, L. Länfumey, M. Chastanet, and J. Adrien. (1991) Regulation of noradrenergic coerulean neuronal firing mediated by 5-HT 2 re:eptors: Involvement of the prepositus hypoglossal nucleus. Neuropharmacology 30:1309. 
172. Gothert, M. and H. Huth. (1980) $\alpha$-Adrenoceptor-mediated modulation of 5-HT release from rat brain cortex slices. Naunyn-Szhmiedeberg's Arch. Pharmacol. 313:21.

173. Gobthert, M. and G. J. Molderings. (1991) Involvement of presynaptic imidazoline receptors in the $\alpha_{2}-$ adrenoceptor-independent inhibition of noradrenaline release by imidazoline derivatives. Naunyn-Schmiedeberg's Arch. Pharmacol. 343:271.

174. Gothert, M., E. Schlicker, and F. Kostermann. (1983) Relationship between transmitter uptake inhibition and effects of alpha-adrenoceptor agonists on serotonin and noradrenaline release in the rat brain cortex. NaunynSchmiedeberg's Arch. Pharmacol. 322:121.

175. Guthert, M. (1980) Serotonin-receptor-mediated modulation of Ca2+-dependent 5-hydroxytryptamine release from neurones of the rat brain cortex. Naunyn-Schmiedeberg's Arch. Pharmacol. 314:223.

176. Graeff, F. G., E. A. Audi, S. S. Almeida, E. O. Graeff, and M. H. Hunziker. (1990) Behavioral effects of 5-HT receptor ligands in the aversive brain stimulation, elevated plus-maze and learned helplessness tests. Neurosci. Biobehav. Rev. 14:501.

177. Graham, A. W. and G. K. : ghajanian. (1971) Effects of amphetamine on single cell activity in a catecholamine nucleus, the locus coeruleus. Nature 234:100.

178. Gravel, P. and C. de Montigny. (1987) Noradrenergic denervation prevents sensitization of rat forebrain neurons to serotonin by tricyclic antidepressant treatment. Synapse 1:233.

179. Gray, J. A. (1987) The neuropsychology of anxiety: An enquiry into the functions of the septo-hippocampal system. Oxford University Press, Oxford.

180. Gray, R. and D. Johnston. (1987) Noradrenaline and $\beta$-adrenoceptor agonists increase activity of voltage-dependent calcium channels in hippocampal neurons. Nature 327:620.

181. Green, A. R. and J. F. W. Deakin. (1980) Brain noradrenaline depletion prevents ECS-induced enhancement of serotonin and dopamine mediated behaviour. Nature. 285:232.

182. Green, A. R. and D. G. Grahame-Smith. (1976) (-)-Propranolol inhibits the behavioural responses of rats to increased 5-hydroxytryptamine in the central nervous system. Nature 262:594.

183. Gross, G., M. Gothert, U. Glapa, G. Engel, and H-J. Schümann. (1985) Lesioning of serotonergic and noradrenergic nerve fibres of the rat brain does not decrease binding of $3 \mathrm{H}$-clonidine or $3 \mathrm{H}$-rauwolscine to cortical membranes. Naunyn-Schmiedeberg's Arch. Pharmacol. 328:229.

184. Grossman, C. J., G. J. Kilpatrick, and K. T. Bunce. (1993) Developmerti of a radioligand binding assay for 5-HT 4 receptors in guinea-pig and rat brain. Br. J. Pharmacol. 109:618.

185. Haas, H. L. and A. Konnerth. (1983) Histamine and noradrenaline decrease calcium-activated potassium conductance in hippocampal pyramidal cells. Nature 302:432.

186. Haddjeri, N., P. Blier, and C. de Montigny. (1994) Effect of the $\alpha_{2}$-adrenoceptor antagonist remeron on rat 5-HT neurotransmission. Soc. Neurosci. Abstr. 20:637.14.

187. Haigler, H. J. and G. K. Aghajanian. (1974) Peripheral serotonin antagonists: failure to antagonize serotonin in brain areas receiving a prominent serotonergic input. J. Neural Transm. 35:157.

188. Hall, M. D., S. el Mestikawy, M. B. Emerit, L. Pichat, M. Hamon, and H. Gozlan. (1985) [3H]8-hydroxy-2-(di-npropylamino)tetralin binding to pre- and postsynaptic 5-hydroxytryptamine sites in various regions of the rat brain. $J$. Neurochem. 44:1685. 
189. Hamblin, M. W., K. Ariani, P. I. Adriaenssens, and R. D. Ciaranello. (1987) [3H]dihydroergotamine as a highaffinity, slowly dissociating radioligand for $5-\mathrm{HT}_{1 \mathrm{~B}}$ binding sites in rat brain membranes: Evidence for guanine nucleotide regulation of agonist affinity states. J. Pharmacol. Exp. Ther. 243:989.

190. Hamblin, M. W., R. W. McGuffin, M. A. Metcalf, D. M. Dorsa, and K. M. Merchant. (1992) Distinct 5-HTIB and 5-HT $1 \mathrm{D}$ serotonin receptors in rat: Structural and pharmacological comparison of the two cloned receptors. Mol. Cell Neurosci. 3:578.

191. Haring, J. H. and J. N. Davis. (1985) Differential distribution of locus coeruleus projections to the hippocampal formation: Anatomical and biochemical evidence. Brain Res. 325:366.

192. Harley, C., J. S. Milway, and J. C. Lacaille. (1989) Locus coeruleus potentiation of dentate gyrus responses: evidence for two systems. Brain Res. Bull. 22:643.

193. Harley, C. W. (1987) A role for norepinephrine in arousal, emotion and learning?: limbic modulation by norepinephrine and the Kety hypothesis. Prog. Neuropsychopharmacol. Biol. Psychiatry 11:419.

194. Harley, C. W. and J. S. Milway. (1986) Glutamate cjection in the locus coeruleus enhances the perforant pathevoked population spike in the dentate gyrus. Exp. Brain Res. 63:143.

195. Harrison, J. K., W. R. Pearson, and K.R. Lynch. (1991) Molecular characterization of $\alpha_{1}$ - and $\alpha_{2}$-adrenoceptors. Trends Pharmacol. Sci. 12:62.

196. Hartig, P., N. Adham, J. Bard, A. Hou-Yu, H. -T. Kao, M. Macchi, L. Schechter, D. Urquhart, J. Zgombick, R. Durkin, R. Weinshank, and T. Branchek. (1993) Molecular biology of 5-HT, receptors. In Serotonin: From cell biology to pharmaculogy and therapeutics. P. M. Vanhoutte, P. R. Saxens, R. Paoletti, N. Brunello, and A. S. Jackson, editors. Kluwer Acad., Dordrecht. 21-31.

197. Hausdorff, W. P., M. G. Caron, and R. J. Lefkowitz. (1990) Tuming off the signal: desensitization of $\beta$ adrenergic receptor function. FASEB J. 4:2881.

198. Hauser, K., H. R. Olpe, and R. S. Jones. (1985) Trimipramine, a tricyclic antidepressant exerting atypical actions on the central noradrenergic system. Eur. J. Pharmacol. 111:23.

199. Hayakawa, H., M. Shimizu, and S. Yamawaki. (1992) The effects of electroconvulsive shock or imipramine on subtypes of al-adrenoceptors in the frontal cortex of the rat. Neuropharmacology 31:955.

200. Heal, D. J., S. A. Butler, M. R. Prow, and W. R. Buckett. (1993) Quantification of presynaptic $\alpha_{2}$-adrenoceptors in rat brain after short-term DSP-4 lesioning. Eur. J. Pharmacol. 249:37.

201. Heal, D. J., E. M. Hurst, M. R. Prow, and W. R. Buckett. (1990) An investigation of the role of 5hydroxytryptamine in the attenuation of presynaptic $\alpha_{2}$-adrenoceptor-mediated responses by antidepressant treatments. Psychopharmacology 101:100.

202. Heal, D. J., J. Philpot, K. M. O'Shaughnessy, and C. L. Davies. (1986) The influence of central noradrenergic function on 5-HT2-ms-jiated head-twitch responses in mice: possible implications for the actions of antidepressant drugs. Psychopharmacology 89:414.

203. Heal, D. J., M. R. Prow, and W. R. Buckett. (1988) Clear evidence that clonidine hypoactivity and mydriasis are mediated by pre- and post-synaptic $\alpha_{2}$-adrenoceptors respectively. Br.J. Pharmacol. 95:781p.

204. Heal, D. J., M. R. Prow, and W. R. Buckett. (1991) Effects of antidepressant drugs and electroconvulsive shock on pre- and postsynaptic $\alpha_{2}$-adrenoceptor function in the brain: rapid down-regulation by sibutramine hydrochloride. Psychopharmacology 103:251. 
205. Heal, D. J., M. R. Prow, J. Gosden, G. P. Luscombe, and W. R. Buckett. (1992) A comparison of various antidepressant drugs demonstrates rapid desensitisation of $\alpha_{2}$-adrenoceptors exclusively by sibutramine hydrochloride. Psychopharmacology 107:497.

206. Hensler, J. G., G. B. Kovachich, and A. Frazer. (1991) A quantitative autoradiographic study of serotonin 1 A receptor regulation. Effect of 5,7-dihydroxytryptamine and antidepressant treatments. Neuropsychopharmacology $4: 131$.

207. Hensler, J. G., G. A. Ordway, C. Gambarana, P. Areso, and A. Frazer. (1991) Serotonergic neurons do not influence the regulation of $\beta$-adrenoceptors induced by either desipramine or isoproterenol. J. Pharmacol. Exp. Ther. 256:656.

208. Hertting, G. and C. Allgaier. (1988) Participation of protein kinase C and regulatory G proteins in modulation of the evoked noradrenaline release in brain. Cell. Mol. Neurobiol. 8:105.

209. Heym, J., M. E. Truison, and B. L. Jacobs. (1981) Effects of adrenergic drugs on raphe unit activity in freely moving cats. Eur. J. Pharmacol. 74:117.

210. Hibert, M. and D. N. Middlemiss. (1986) Stereoselective blockade at the 5-HT autoreceptor and inhibition of radioligand binding to central 5-HT recognition sites by the optical isomers of methiothepin. Neuropharmacology 25:1.

211. Hieble, J. P. and R. R. Ruffolo. (1992) Imidazoline receptors: Historical perspective. Fundam. Clin. Pharmacol. 6:7s.

212. Hjorth, S. and T. Magnusson. (1988) The 5-HTIA receptor agonist, 8-OH-DPAT, preferentially activates cell body 5-HT autoreceptors in rat brain in vivo. Naunyn-Schmiedeberg's Arch. Pharmacol. 338:463.

213. Hjorth, S. and R. Tao. (1991) The putative 5-HT $1 B$ receptor agonist CP-93,129 suppresses rat hippocampal 5-HT release in vivo: comparison with RU 24969. Eu-. J. Pharmacol. 209:249.

214. Hollingsworth, P. J. and C. B. Smith. (1991) Factors influencing the finction of presynaptic $\alpha_{2}$-adrenoceptors in rat brain. In Presynaptic receptors and neuronal transporters. S. Z. Langer, A. M. Galzin, and J. Costentin, editors. Pergamon, Oxford. 39-42.

215. Hopkins, W. F. and D. Johnston. (1984) Frequency-rependent noradrenergic modulation of long-term potentiation in the hippocampus. Science 226:350.

216. Hopkins, W. F. and D. Johnston. (1988) Noradrenergic enhancement of long-term potentiation at mossy fiber synapses in the hippocampus. J. Neurophysiol. 59:667.

217. Hosoda, K. and R. S. Duman. (1993) Regulation of $\beta_{1}$-adrenergic receptor mRNA and iigand binding by antidepressant treatments and norepinephrine depletion in rat frontal cortex. I. Neurochem. 60:1335.

218. Hotta, I. and S. Yamawaki. (1988) Possible involvement of presynaptic 5-HT autoreceptors in effect of lithium on 5-HT release in hippocampus of rat. Neuropharmacology 27:987.

219. Hoyer, D. (1990) Serotonin 5-HT3, 5-HT4, and 5-HT-M receptors. Ni.uropsychopharmacology. 3:371.

220. Hoyer, D. (1991) The 5-HT receptor family: Ligands, distribution and receptor-effector coupling. In S-HT $1 \mathrm{~A}$ agonists, S-HT3 antagonists and benzodiazepines: their comparative behavioural pharmacology. R. J. Rodgers and S. J. Cooper, editors. Wiley, Chichester. 31.

221. Hoyer, D., G. Engel, and H. O. Kalkman. (1985) Characterization of the 5-HT/B recognition site in rat brain: Binding studies with (-)[125]iodocyanopindolol. Eur. J. Pharmacol. 118:1. 
222. Hoyer, D., A. Pazos, A. Probst, and J. M. Palacios. (1986) Serotonin recepiors in the human brain. I. Characterization and autoradiographic localization of 5-HT $1 \mathrm{~A}$ recognition sites. Apparent absence of 5-HT, $1 \mathrm{~B}$ recognition sites. Brüin Res. 376:85.

223. Hoyer, D., P. Schoeffter, C. Waeber, and J. M. Palacios. (1990) Serotonin 5-HTID receptors. Ann. NY Acad. Sci. 600:168.

224. Hsiao, J. K., H. Ágren, J. J. Bartko, M. V. Rudorfer, M. Linnoila, and W. Z. Potter. (1987) Monoamine neurotransmitter interactions and the prediction of antidepressant response. Arch. Gen. Psychiatry 44:1078.

225. Huang, Y. H., J. W. Maas, and G. H. Hu. (1980) The time course of noradrenergic pre- and postsynaptic activity during chronic desipramine treatment. Eur. J. Pharmacol. 68:41.

226. Humplirey, P. P. A., P. Hartig, and D. Hoyer. (1993) A reappraisal of 5-HT receptor classification. In Serotonin: From cell biology to pharmacology and therapeutics. P. M. Vanhoutte, P. R. Saxena, R. Paoletti, N. Brunello, and A. S. Jackson, editors. Kluwer Acad., Dordrecht. 41-47.

227. Hyttel, J., K. F. Overo, and J. Amt. (1984) Biochemical effects and drug levels in rats after long-term treatment with the specific 5-HT-uptake inhibitor, citalopram. Psychopharmacology 83:20.

228. Innis, R. B., E. J. Nestler, and G. K. Aghajanian. (1988) Evidence for G protein mediation of serotonin- and GABAB-induced hyperpolarization of rat dorsal raphe neurons. Brain Res. 459:27.

229. Jackisch, R., H. Y. Huang, H. Rensing, D. Lauth, C. Allgaier, and G. Hertting. (1992) $\alpha_{2}$-Adrenoceptor mediated inhibition of exocytotic noradrenaline release in the absence of extracellular calcium. Eur. J. Pharmacol. Mol. Pharmacol. 226:245.

230. Jackisch, R., E. Werle, and G. Hertting. (1984) Identification of mechanisms involved in the modulation of release of noradrenaline in the hippocampus of the rabbit in vitro. Neuropharmacology 23:1363.

231. Jacobs, B. L., E. D. Abercrombie, C. A. Fomal, E. S. Levine, D. A. Morilak, and I. L. Stafford. (1991) Singleunit and physiological analyses of brain norepinephrine function in behaving animals. Prog. Brain Res. 88:159.

232. Jacobs, B. L. and E. C. Azmitia. (1992) Structure and function of the brain serotonin system. Physiol. Rey. $72: 165$.

233. Jacobs, B. L., L. O. Wilkinson, and A. F. Casimir. (1990) The role of brain serotonin:A neurophysiologic perspective. Neuropsychopharmacolog.ty 3:473.

234. Jahnsen, H. (1980) The action of 5-hydroxytryptamine on neuronal membranes and synaptic transmission in area CA $A_{1}$ of the hippocampus in vitro. Brain Res. 197:83.

235. Janowsky, D. S., L. R. Steranka, D. D. Gillespie, and F. Sulser. (1982) Role of neuronal signal input in the downregulation of central noradrenergic receptor function by antidepressant drugs. J. Neurochem. 39:290.

236. Jin, H., D. Oksenberg, A. Ashkenazi, S. J. Peroutka, A. M. V. Duncan, R. Rozmahel, Y. Yang, G. Mengod, J. M. Palacios, and B. F. O'Dowd. (1992) Characterization of the human 5-hydroxytryptaminejB receptor. J. Biol. Chem. 267:5735.

237. Joels, M., G. Bouma, W. Hesen, and Y. Zegers. (1991) Increased effect of noradrenaline on synaptic responses in rat $\mathrm{CA}_{1}$ hippocampal area after adrenalectomy. Brain Res. 550:347.

238. Joels, M. and E. R. de Kloet. (1991) Effect of corticosteroid hormones on electrical activity in rat hippocampus. J. Steroid Biochem. Molec. Biol. 40:83.

23\%. Johnson, D. S. and S. F. Heinemann. (1992) Cloning and expression of the rat 5HT 3 receptor reveals speciesspecific sensitivity to curare antagonism. Soc. Neurosci. Abstr. 18:265.1. 
240. Johnson, J., ‥ Sherman, F. Petty, D. Taylor, and F. Henn. (1982) Receptor changes in learned helplessness. Soc. Neurosci. Abst. 8:392.

241. Jones, B. E. and R. Y. Moore. (1977) Ascending projections of the locus coeruleus in the rat. II. Autoradiographic, study. Brain Res. 127:23.

242. Jones, G. H., T. W. Robbins, and C. A. Marsden. (1989) Isolation-rearing retards the acquisition of scheduleinduced polydipsia in rats. Physiol. Behav. 45:71.

243. Kahn, R. J., D. M. McNair, R. S. Lipman, L. Covi, K. Rickels, R. Downing, S. Fisher, and L. M. Frankenthaler. (1986) Imipramine and chlordiazepoxide ir. depressive and anxiety disorders. Il. Efficacy in anxious outpatients. Arch. Gen. Psychiatry 43:79.

244. Kahn, R. S., H. M. van Praag, S. Wetzler, C. M. Asnis, and G. Barr. (1988) Serutonin and anxiety revisited. Biol. Psychiatry 23:i89.

245. Kalen, P., E. Rosegren, O. Lindvall, and A. Bjorklund. (1989) Hippocampal noradrenaline and serotonin release over 24 hours as measured by the dialysis technique in freely moving rats: correlation to behavioural activity state, effect of handling and tail-ptach. Eur. J. Neurosci. 1:181.

246. Kellar, K. J. and D. A. Beigstrom. (1983) Electroconvulsive shock: Effects on biochemical correlates of neurotransmitter receptors in rat brain. Neuropharmacology 22:401.

247. Kilpatrick, G. J., B. J. Jones, and M. B. Tyers. (1988) The distributiun uí specific binding of the 5-HT3 receptor ligand 3HGR65630 in rat brain using quantitative autoradiography. Neurosci. Lett. 94:156.

248. Kohler, C., A.-C. Radesater, W. Lang, and V. Chan-Palay. (1980) Distribution of serotonin-1A receptors in the monkey and the postmortem human hippocampal region. A quantitative autoradiographic study using the selective agonist [3H]8-OH-DPAT. Neurosci. Lett. 72;43.

249. Kolasa, K., L. Song, and R. S. Jope. (1992) Adrenalectomy increases phosphoinositide hydrolysis induced by norepinephrine or excitatory amino acids in rat hippocampal slices. Brain Res. 579:128.

250. Koslow, J. H., J. W. Maas, C. L. Bowden, J. M. Davis, I. Hanin, and I. Javaid. (1983) CSF and urinary biogenic amines and metabolites in depression and mania. Arch. Gen. Psychiatry 40:999.

251. Kovarhich, G. B., A. Frazer, and C. E. Aronson. (1993) Effect of chronic administration of antidepressants on $\alpha_{2}$-adrenoceptors in the locus coeruleus and its projection fields in rat brain determined by quantitative autoradiography. Neuropsychopharmacology 8:57.

252. Koyama, Y. and Y. Kayama. (1993) Mutual interactions among cholinergic, noradrenergic and serotonergic neurons studied by ionophoresis of these transmitters in rat brainstem nuclei. Neuroscience 55:1117.

253. Laborit, H. (1973) Les comportements. Biologie, physiologie, pharmacologie. Masson, Paris.

254. Lac.ille, J. C. and C. W. Harley. (1985) The action of norepinephrine in the dentate gyrus: $\beta$-mediated facilitation of evoked potentials in vitro. Brain Res. 358:210.

255. Lacroix, D., P. Blier, O. Curet, and C. de Montigny. (1991) Effects of long-term desipramine administration on noradrenergic neurotransmission reuptake: Electrophysiological studies in the rat brain. J. Pharmacol. Exp. Ther. 257:1081.

256. Lake, C. R., D. Pickar, M. Ziegler, S. Lipper, S. Slater, and D. L. Murphy. (1982) High plasma norepinephrine levels in patients with major affective disorder. Am. J. Psychiatry 139:1315.

257. Lanfumey, L. and J. Adrien. (1988) Adaptive changes of $\beta$-adrenergic receptors after neonatal locus coeruleus lesion: regulation of serotoninergic unit activity. Synapse 2:644. 
258. Langer, S. Z. (1974) Presynaptic regulation of catecholamine release. Biochem. Pharmacol. 23:1793.

259. Langer, S. Z. and C. Moret. (1982) Citalopram antagonizes the stimulation by lysergic acid diethylamide of presynaptic inhibitory serotonin autoreceptors in the rat hypothalamus. J. Pharmacol. Exp. Ther. 222:220.

260. Langley, J. N. (1901) Observations on the physislogical action of extracts of the supra-renal bodies. J. Physiol. (Lond.) 27:237.

261. Langmoen, I. A.. M. Segal, and P. Andersen. (1981) Mechanisms of norepinephrine actions on hippocampal pyramidal Arch. Gen. Psychia!ry 40:999.

251. Kovachich, G. B., A. Frazer, and C. E. Aronson. (1993) Effect of chronic administration of antidepressants on $\alpha_{2}$-adrenoceptors in the locus coeruleus and its projection fields in rat brain determined by quantitative autoradiography. Neuropsychopharmacology 8:57.

252. K.cjama, Y. and Y. Kayama. (1993) Mutual interactions among cholinergic, noradrenergic and serotonergic neurons studied by ionophoresis of these transmitters in rat brainstem nuclei. Neuroscience 55:1117.

253. Laborit, H. (1973) Les comportements. Biologie, physiologie, pharmacologie. Masson, Paris.

254. Lacaille, J. C. and C. W. Harley. (1985) The action of norepinephrine in the dentate gyrus: $\beta$-mediated facilitation of evoked potentials in vitro. Brain Res. 358:210.

255. Lacroix, D., P. Blier, O. Curet, and C. de Montigny. (1991) Effects of long-term desipramine administration on noradrenergic neurotransmission reuplake: Electrophysiological studies in the rat brain. J. Pharmacol. Exp. Ther. 257:

256. Lake, C. R., D. Pickar, M. Ziegier, S. Lipper, S. Slater, and D. L. Murphy. (1982) High plasma norepinephrine leveis in patients with major affective disorder. Am. J. Psychiatry 139:1315.

257. Lanfumey, L. and J. Adrien. (1988) Adaptive changes of $\beta$-adrenergic receptors after neonatal locus cocruleus lesion: regulation of serotoninergic unit activity. Synapse 2:644.

258. Langer, S. Z. (1974) Presynaptic regulation of catecholamine release. Biochem. Pharmacol. 23:1793.

259. Langer, S. Z. and C. Moret. (1982) Citalopram antagonizes the stimulation by lysergic acid diethylamide of presynaptic inhibitory serotonin autoreceptors in the rat hypothalamus. J. Pharmacol. Exp. Ther. 222:220.

260. Langley, J. N. (1901) Observations on the physiological action of extracts of the supra-renal bodies. J. Physiol. 27:237.

261. Langmoen, I. A., M. Segal, and P. Andersen. (1981) Mechanisms of norepinephrine actions on hippocampal pyramidal cells in vitro. Brain Res. 208:349.

262. Lapin, I. P. and G. F. Oxenkrug. (1969) Intensification of central serotonergic processes as a possible determinant of the thymoleptic effect. Lancet 1:132.

263. Lechin, F., B. van der Dijs, J. Amat, and M. Lechin. (1989) Neurochemistry and clinical disorders: circuitry of some psychiatric and psychosomatic syndromes. F. Lechin and B. van der Dijs, editors. CRC, Boca Rato.

264. Leger, L. and L. Descarries. (1978) Serotonin nerve terminals in the locus coeruleus of adult rat: a radioautographic study. Brain Res. 145:1.

265. Lesch, K.P. and H. K. Manji. (1992) Signal-transducing G proteins and antidepressant drugs: Evidence for modulation of a subunit gene expression in rat brain. Biol. Psychiatry 32:549.

266. Leung, P. P. and J. J. Miller. (1988) Dual role of norepinephrine in the hippocampal CA $A_{1}$ region of the rat: inhibition and disinhibition. Can. J. Physiol. Pharmacol. 66:814. 
267. Levith, P., J. E. Pintar, and X. O. Breakefield. (1982) Immunocytochemical demonstration of monoamine oxidase $B$ in brain astrocytes and serotonergic neurons. Proc. Nall. Acad. Sci. U. S A. 79:6385.

268. Lewandowsky, M. (1898) Ueber eine wirkung des nebennierenextractes auf das auge. Zentralbl. Physiol. , 1 liz.599.

269. Leysen, J. E. (1990) Gaps and peculiarities in 5-HT2 receptor studies. Neuropsychopharmacology 3:361.

270. Li, G., S. Regunathan, C. J. Barrow, J. Eshraghi, R. Cooper, and D. J. Reis. (1994) Agmatine: An endogenous clonidine-displacing substance in the brain. Science 263:966.

271. Limberger, N., G. Bonanno, L. Spath, and K. Starke. (1986) Autoreceptors and $\alpha_{2}$-adrenoceptors at the serotonergic axons of rabbit brain cortex. Naunyn-Schmiedeberg's Arch. Pharmacol. 332:324.

272. Lindvall, O. and A. Bjorklund. (1974) The organization of the ascending catecholamine neuron systems in the rat brain as revealed by glyoxylic acid fluorescence method. Acta Psychiatr. Scand. 412:1.

273. Loizou, L. A. (1969) Projections of the nucleus locus coeruleus in the albino rat. Brain Res. 15:563.

274. Lucki, I. and A. Frazer. (1982) Prevention of the serotonin syndrome in rats by repeated administration of monoamine oxidase inhibitors but not tricyclic antidepressants. Psychopharmacology 77:205.

275. Lum, J. T. and M. F. Piercey. (1988) Electrophysiological evidence that spiperone is an antagonist of 5-HT $1 \mathrm{~A}$ receptors in the dorsal raphe nucleus. Eur. J. Pharmacol. 149:9.

276. Maas, J. W. (1975) Biogenic amines and depression. Arch. Gen. Psychiatry 32:1357.

277. MacKinnon, A. C., M. Spedding, and C. Brown. (1994) $\alpha_{2}$-Adrenoceptors: More subtypes but fewer functional differences. Trends Pharmacol. Sci. 15:119.

278. MacRae-Deguerce, A., B. Berod, A. Mermet, A. Keller, G. Chouvet, T. Joh, and J. Pujol. (1982) Alterations in tyrosine hydrolase activity elicited by raphe nuclei lesions in the rat locus coeruleus: Evidence for the involvement of serotonin afferents. Brain Res. 235:285.

279. Madison, D. V. and R. A. Nicoll. (1982) Noradrenaline blocks ascommodation of pyramidal cell discharge in the hippocampus. Nature 299:636.

280. Madison, D. V. and R. A. Nicoll. (1986) Cyclic adenosine 3',5'-monophosphate mediates $\beta$-receptor actions of noradrenaline in rat hippocampal pyramidal cells. J. Physiol. (Lond) 372:245.

281. Madison, D. V. and R. A. Nicoll. (1986) Actions of noradrenaline recorded intracellularly in rat hippocampal CA 1 pyramidal neurones, in vitro. J. Physiol. (Lond) 372:221.

232. Madison, D. V. and R. A. Nicoll. (1988) Norepinephrine decreases synaptic inhibition in the rat hippocampus. Brain Res. 442:131.

283. Maj, J., V. Klimek, and G. Nowak. (1985) Antidepressant drugs given repeatedly increase binding to $\alpha_{1-}$ adrenoceptors in the rat cortex. Eur. J. Pharmacol. 119:113.

284. Man ounas, L. A., C. A. Mullen, E. O'Hearn, and M. E. Molliver. (1991) Dual serotonergic projections to forebrain in the rat: Morphologically distinct 5-HT axon terminals exhibit differential vulnerability to neurotoxic amphetamine derivatives. J. Comp. Neurol. 314:558.

285. Manier, D. H., D. D. Gillespie, L. R. Steranka, and F. Sulser. (1984) A pivotal role for serotonin in the downregulation of $\beta$-adrenoceptors by antidepressants: Reversibility of the action of p-chlorophenylalanine. Experientia 40:1223. 
286. Manier, D. H., D. D. Gillespie, and F. Sulser. (1989) Dual aminergic regulation of central $\beta$-adrenoceptors. Effect of "atypical" antidepressants and 5-hydroxytryptophan. Neuropsychopharmacology. 2:89.

287. Marciani, M. G., P. Calabresi, P. Stanzione, and G. Bernardi. (1984) Dopaminergic and norkirenergic responses in the hippocampal slice preparation. Evidence for different receptors. Neuropharmacology 23:303.

288. Maricq, A., A. S. P.ièišñn, A.. I. Rrake, R. M. Myers, and D. Julius. (1991) Primary structure and functional expression of the 5-!iT3 recistor, a serotonin gated ion channel. Science 254:432.

289. Marksiein, R., K. Digges, N. R. Marshall, and K. Starke. (1984) Forskolin and the release of noradrenaline in cerebrocortical slices. Naunyn-Schmiedeberg's Arch. Pharmacol. 325:17.

290. Martin, K. F., S. Hannon, I. Phillips, and D. J. Heal. (1992) Opposing roles for 5-HT 1 B and $5 \cdot \mathrm{HT}_{3}$ receptors in the control of 5-HT release in rat hippocampus in vivo. Br. J. Pharmacol. 106:139.

291. Marwaha, J. and G. K. Aghajanian. (1982) Relative potencies of alpha-1 and alpha-2 antagonists in the locus ceruleus, dorsal raphe and dorsal lateral geniculate nuclei: an electrophysiological study. J. Pharmacol. Exp. Ther. 222:287.

292. Masuko, S., Y. Nakajima, S. Nakajima, and K. Yamaguchi. (1986) Noradrenergic neurons from the locus coeruleus in dissociated cell culture: Culture methods, morphology, and electrophysiology. J. Neurosci. 6:3229.

293. Maura, G., G. Bonanno, and M. Raiteri. (1992) Presynaptic $\alpha_{2}$-adrenoceptors mediating inhibition of noradrenaline and 5-HT release in rat cerebral cortex: further characterization as different $\alpha_{2}$-adrenoceptor subtyptes. Naunyn-Schmiedeberg's Arch. Pharmacol. 345:410.

294. Maura, G., A. Gemignani, and M. Raiteri. (1982) Noradrenaline inhibits central serotonin release through $\alpha_{2}-$ adrenoceptors located on serotonergic nerve terminals. Naunyn-Schmiedeberg's Arch. Pharmacol. 320:272.

295. Maura, G., E. Roccatagliata, and M. Raiteri. (1986) Serotonin autoreceptor in rat hippocampus: pharmacological characterization as a subtype of the 5-HT, receptor. Naunyn-Schmiedeberg's Arch. Pharmacol. 334:323.

296. Maura, G., M. Ulivi, and M. Raiteri. (1987) (-)-Propranolol and (+/-)-cyanopindolol are mixed agonists-antagonists at serotonin autoreceptors in the hippocampus of the rat brain. Neuropharmacology 26:713.

297. McKenna, K. F., G. B. Baker, R. T. Coutts, and A. J. Greenshaw. (1992) Chronic administration of the antidepressant-antipanic drug phenelzine and its $\mathrm{N}$-acetylated analogue: Effects on monoamine oxidase, biogenic amines, and $\alpha_{2}$-adrenoceptor function. J. Pharm. Sci. 81:832.

298. McMillen, B. A., $\ddot{w}$. Warnack, D. C. German, and P. A. Shore. (1980) Effects of chronic desipramine treatment on rat brain noradrenergic responses to $\alpha$-adrenergic drugs. Eur. J. Pharmacol. 61:239.

299. McRae-Degueurce, A., L. Léger, L. Wiklund, and J. F. Pujol. (1981) Functional recuperation of the serotonergic innervation in the rat locus coeruleus. J. Physiol. 77:389.

300. Melia, K. R., K. Rasmussen, R. Z. Terwilliger, J. W. Haycock, E. J. Nestler, and R. S. Duman. (1992) Coordinate regulation of the cyclic AMP system with firing rate and expression of tyrosine hydroxylase in the rat locus coeruleus: effects of chronic stress and drug treatments. J. Neurochem. 58:494.

301. Mendelson, S. D. and B. S. McEwen. (1992) Autoradiographic analyses of the effects of adrenalectomy and corticosterone on 5-HTIA and 5-HT 1 B receptors in the dorsal hippocampus and cortex of the rat. Neuroendocrinology. 55:444.

302. Menkes, D. B. and G. K. Aghajanian. (1981) $\alpha_{1}$-Adrenoceptor-mediated responses in the lateral geniculate nucleus are enhanced by chronic antidepressant treatment. Eur. J. Pharmacol. 74:27. 
303. Menkes, D. B., G. K. Aghajanian, and D. W. Gallager. (1983) Chronic antidepressant treatment enhances agonist affinity of brain $\varkappa_{1}$-adrenoceptors. Eur. J. Pharmacol. 87:35.

304. Menkes, D. B., G. K. Aghajanian, and R. B. McCall. (1980) Chronic antidepressant treatment enhances $\alpha$ adrenergic and serotonergic responses in the facial nucleus. Life Sci. 27:45.

305. Michal, G. (1982) Biochemical pathways. Boehringer Mannheim.

306. Middlemiss, D. N. (1986) Blockade of the central 5-HT autoreceptor by $\beta$-adrenoceptor antagonists. Eur. J. Pharmacol. 120:51.

307. Middlemiss, D. N., M. E. Bremer, and S. M. Smith. (1988) A pharmacological analysis of the 5-HT receptor mediating inhibition of 5.HT release in the guinea-pig frontal cortex. Eur. J. Pharmacol. 157:101.

308. Middlemiss, D. N. and P. H. Hutson. (1990) The 5-HT1B receptors. Ann. N. Y. Acad. Sci. 600:132.

309. Minneman, K. P., M. D. Dibner, B. B. Wolfe, and P. B. Molinoff. (1979) $\beta_{1}$ - and $\beta_{2}$-adrenergic receptors in rat cercbral cortex are independently regulated. Science 204:866.

310. Mizuta, T. and T. Segawa. (1988) Chronic effects of imipramine and lithium on postsynaptic 5-HT $1 \mathrm{~A}$ and 5$\mathrm{HT}_{1 \mathrm{~B}}$ sites and on presynaptic 5-HT3 sites in rat brain. Jpn. J. Pharmacol. 47:107.

311. Molderings, G. J. and M. Gothert. (1990) Mutual interaction between presynaptic $\alpha_{2}$-adrenoceptors and 5-HT 18 receptors on the sympathetic nerve terminals of the rat inferior vena cava. Naunyn-Schmiedeberg's Arch.Pharmacol. 341:391.

312. Mongeau, R. (1990) Antidepressant and anxiolytic action on the serotonin $1 \mathrm{~A}$ binding site. Masters thesis at McGill University, Montreal.

313. Mongeau, R., S. A. Welner, R. Quirion, and B. E. Suranyi-Cadotte. (1992) Further evidence for differential affinity states of serotonin IA receptor in rat hippocampus. Brain Res. 590:229.

314. Moore, R. Y., A. E. Halaris, and B. E. Jones. (1978) Serotonin neurons of the midbrain raphe: ascending projections. J. Comp. Neurol. 180:417.

315. Moret, C. and M. Briley. (1990) Serotonin autoreceptor subsensitivity and antidepressant activity. Eur. J. Pharmacol. 180:351.

316. Morin, D., R. Sapena, R. Zini, and J. P. Tillement. (1992) Serotonin enhances the $\beta$-adrenergic response in rat brain cortical slices. Eur. J. Pharmacol. 225:273.

317. Mueller, A. L., B. J. Hoffer, and T. V. Dunwiddie. (1981) Noradrenergic responses in rat hippocampus: evidence for mediation by $\alpha$-and $\beta$-receptors in the in vitro slice. Brain Res. 214:113.

318. Mueller, A. L., K. L. Kirk, B. J. Hoffer, and T. V. Dunwiddie. (1982) Noradrenergic responses in rat hippocampus: electrophysiological actions of direct- and indirect-acting symnathomimetics in the in vitro slice. $J$. Pharmacol. Exp. Ther. 223:599.

319. Mueller, A. L.. M. R. Palmer, B. J. Hoffer, and T. V. Dunwiddie. (1982) Hippocampal noradrenergic responses in vivo and in vitro. Characterization of alpha and beta components. Naunyn-Schmiedeberg's Arch. Pharmacol. 318:259.

320. Mynlieff, M. and T. V. Dunwiddie. (1988) Noradrenergic depression of synaptic responses in hippocampus of rat: evidence for mediation by $\alpha_{1}$-receptors. Neuropharmacology $27: 391$.

321. Nalepa, I. and J. Vetulani. (1993) Enhancement of the responsiveness of cortical adrenergic receptors by chronic administration of the 5-hydroxytryptamine uptake inhibitor citalnpram. J. Neurochem. 60:2029. 
322. Nelson, J. C., C. M. Mazure, M. B. Bowers, and P. I. Jatlow. (1991) A preliminary, open study of the combination of fluoxetine and desipramine for rapid treatment of major depression. Arch. Gen. l'sychiurry 48.303.

323. Nestler, E. J., A. McMahon, E. L. Sabban, J. F. Tallman, and R. S. Duman. (1990) Cirronic antidepressant adruinistration decreases the expression of tyrosine hydroxylase in the rat locus coeruleus. Proc. Natl. Acad. Sci. U.S A. 87:7522.

324. Newman, M. E., D. Drummer, and B. Lerer. (1990) Single and combined effects of desipramine and lithium on serotonergic receptor number and second messenger function in rat brain. J. Pharmacol. Exp. Ther. 260:16.

325. Newman, M. E. and B. Lerer. (1988) Chronic electroconvulsive shock and desipramine reduce the degree of inhibition by 5 -HT and carbachol of forskolin-stimulated adenylate cyclase in rat hippocampal membranes. Eur. $J$. Pharmacol. 148:257.

326. Newman, M. E. and B. Lerer. (1989) Modulation of second messenger function in rat brain by in vivo altcration of receptor sensitivity: relevance to the mechanism of action of electroconvulsive therafy and antidepressants. Prog. Neuropsychopharmacol. Biol. Psychiatry 13:1.

327. Newman, M. E., I. Miskin, and B. Lerer. (1987) Effects of single and repeated electroconvuisive shock administration on inositol phosphate accumulation in rat brain slices. J. Neurochem. 49:19.

328. Newman, M. E., B. Shapira, and B. Lerer. (1992) Regulation of 5-hydroxytryptamine 1 A receptor function in rat hippocampus by short- and long-term administration of 5-hydroxytryptamine 1A agonist and antidepressants. $J$. Pharmacol. Exp. Ther. 260:16.

329. Nichols, A. J. (1991) $\alpha$-Adrenoceptor signal transduction mechanism. In $\alpha$-adrenoceptors: Molecular biology, Biochemistry and Pharmacology. Prog. Basic Clin. Pharmacol. R. R. Ruffolo, editor. Krager Press, Basel , 44-74.

330. Nisenbaum, L. K., M. J. Zigmond, A. F. Sved, and E. D. Abercrombie. (1991) Prior exposure to chronic stress results in enhanced synthesis and release of hippocampal norepinephrine in resf: nse to a novel stressor. J. Neurosci. $11: 1478$.

331. Nishikawa, T., M. Tanaka, A. Tsuda, Y. Khono, and N. Nagasaki. (1983) Serotonergic-catecholaminergic interactions and footshock-induced jumping behavior in rats. Eur. J. Pharmacol. 94:53.

332. Nowak, G. and E. Przegalinski. (1988) Effect of repeated treatment with antidepressant drugs and electroconvulsive shock (ECS) on [3H] prazosin binding to different rat brain structures. J. Neural Transm. 71:57.

333. Odagaki, Y., T. Koyama, and I. Yamashita. (1991) No alterations in the 5-HT1A -mediated inhibition of forskolin-stimulated adenylate cyclase activity in the hippocampal membranes from rats chronically treated with lithium or antidepressants. J. Neural Transm. Gen. Sect. 86:85.

334. Oleskevich, S. and L. Descarries. (1990) Quantified distribution of the serotonin innervation in adult rat hippocampus. Neuroscience 34:19.

335. Oleskevich, S., L. Descarries, and J. C. Lacaille. (1989) Quantified distribution of the noradrenaline innervation in the hippocampus of adult rat. J. Neurosci. 9:3803.

336. Olpe, H. R., A. Schellenberg, and R. S. Jones. (1984) The sensitivity of hippocampal pyramidal neurons to serotonin in vitro: effect of prolonged treatment with clorgyline or clomipramine. J. Neural Transm. 60:265.

337. Ortmann, R., S. Martin, E. Radeke, and A. Delini-Stula. (1981) Interaction of $\beta$-adrenoceptor agonists with the serotonergic system in rat brain. A behavioral study using the L-5-HTP syndrome. Naunyn-Schmiedeberg's Arch. Pharmacol. 316:225.

338. Otmakhov, N. A. and A. G. Bragin. (1982) Effects of norepinephrine and serotonin upon spontaneous activity and responses to mossy fiber stimulation of CA3 neurons in hippocampal slices. Brain Res. 253:173. 
339. Ozawa, H. and M. M. Rasenick. (1991) Chronic electroconvulsive treatment augments coupling of the GTPbinding protein $G_{3}$ to the catalytic moiety of adenylyl cyclase in a manner similar to that seen with chronic antidepressant drugs. J. Neurochem. 56:330.

340. Ozawa, H., M. M. Rasenick, and T. Saito. (1990) Effect of chronic antidepressant administration on beta adrenergic receptor GTP binding protein adenylate cyclase coupling. Biog. Amines 7:535.

341. Palfreyman, M. G., A. K. Mir, M. Kubina, D. N. Middlemiss, M. Richards, M. D. Tricklebank, and J. R. Fozard. (i986) Monoamine receptor sensitivity changes following chrołac administration of MDL 72394, a site-directed inhibitor of monoamine oxidase. Eur. J. Pharmacol. 130:73.

342. Pang, K. and G. M. P.use. (1987) Differential effects of norepinephrine on hippocampal complex-spike and thetaneurons. Brain Res. 425:146.

343. Passarelli, F., T. Costa, and O. F. Almeida. (1988) Pertussis toxin inactivates the presynaptic serutonin autoreceptor in the hippocampus. Eur. J. Pharmacol. 155:297.

344. Paton, W. D. M. and E. S. Vizi. (1969) The inhibitory action of noradrenaline and acetylcholine otitput by guinea-pig longitudinal muscle strip. Br. J. Pharmacol. 35:10.

345. Paul, I. A., G. E. Duncan, K. R. Powell, R. A. Mue'tr, J. S. Hong, and G. R. Breese. (1988) Regionally specific neural adaptation of $\beta$-adrenergic and 5-hydroxytryptaminez receptors after antidepressant administration in the forced swim test and after chronic antidepressant drug treatment. J. Pharmacol. Exp. Ther. 246:956.

346. Peroutka, S. J. (1986) Pharmacological differentiation and characterization of 5-HT $1 \mathrm{~A}, 5-\mathrm{HT}_{1 \mathrm{~B}}$, and 5-HT $1 \mathrm{C}$ binding sites in rat frontal cortex. J. Neurochem. 47:529.

347. Peroutka, S. J. (1988) 5-HT receptor subtypes: molecular, biochemical and physiological characterization. Trends Neurosci. 11:496.

348. Peroutka, S. J., M. D. Mauk, and J. D. Kocsis. (1987) Modulation of neuronal activity in the hippocampus by 5hydroxytryptamine and 5-hydroxytryptamine1A selective drugs. Neuropharmacology 26:139.

349. Peroukka, S. J. and S. H. Snyder. (1980) Long-term antidepressant treatment decreases spiroperidol-labelled serotonin receptor binding. Science 210:88.

350. Peters, J. A., M. M. Hilary, and J. L. Jeremy. (1992) Recent advances in the electrophysiological characterization of 5-HT3 receptors. Trends Pharmacol. Sci. 13:391.

351. Pickel, V. M., T. H. Joh, and D. J. Reis. (1977) A serotonergic innervation of noradrenergic neurons in nucleus locus coenuleus: demonstration by immunocytochemical localization of the transmitter specific enzymes tyrosine and tryptophan hydroxylase. Brain Res. 131:197.

352. Pineyro, G., C. de Montigny, and P. Blier. (1993) Regulation of somatodendritic release of 5-HT in the rat dorsal maphe by 5-HT ID receptors. Soc. Neurosci. Abstr. 19:93.4.

353. Plaznik, A., W. Danysz, W. Kostowski, A. Bidzinski, and M. Hauptmann. (1983) Interaction between noradrenergic and serotonergic brain systems as evidenced by microinjections of adrenergic agonists and antagonists into the median raphe nucleus. Pharmacol. Biochem. Behav. 19:27.

354. Plaznik, A. and W. Kostowski. (1991) The involvement of serotonin and noratrenaline in the psychopathological processes of stress and depression: Animal models and the effect of antidepressant drugs. Pol. J. Pharmacol. Pharm. 43:301.

355. Quik, M. and E. Azmitia. (1983) Selective destruction of the serotonergic fibers of the fornix-fimbria and cingulum bundle increases 5-HT, but not 5-HT2 receptors in rat midbrain. Eur. J. Pharmacol. 90:377. 
356. Quineaux, N., J. Scuvée-Moreau, and A. Dresse. (1982) Inhibition of in vitro and ex vivo uptake of noradrennline and 5-hydroxytryptamine by five antidepressants; Correlation with reduction of spontaneous firing rate of central monoaminergic neurones. Naunyn-Schmiedeberg's Arch. Pharmacol. 319:66.

357. Raiteri, M., G. Maura. A. Gemignani, and A. Pittaluga. (1983) Differential blockade by (-)mianserin of ute $\alpha_{2}-$ adrenoceptors mediating inhibition of noradrenaline and serotonin release from rat brain synaptosomes. NaunynSchmiedeberg's Arch. Pharmacol. 322:180.

358. Raite:: M., G. Maura, and P. Versace. (1983) Functional evidence for two stereochemically different alpha-2 adrenoceptors regulating central norepinephrine and serotonin release. J. Pharmacol. Exp. Ther. 224:679.

359. Ramdine, R., A. M. Galzin, and S. Z. Langer. (1989) Involvement of adenylate cyclase and protein kinase C in the alpha 2-adrenoceptor-mediated inhibition of noradrenaline and 5-hydroxytryptamine release in rat hypothalaunic slices. Naunyn-Schmiedeberg's Arch. Pharmacol. 340:386.

360. Rasmussen, K., R. A. Glennon, and G. K. Aghajanian. (1986) Phenethylar.ine hallucinogens in the locus coeruleus: potency of action correlates with rank order of 5-HT2 binding affinity. Eur. J. Pharmacol. 132:79.

361. Rasmussen, K. and B. Jacobs. (1986) Single unit activity of locus coeruleus neurons in the freely moving cat. II conditioning and pharmacologic studies. Brain Res. 371:335.

362. Rasmussen, K., D. Morilak, and B. L. Jacobs. (1986) Single unit activity of locus coeruleus neurons in the freely moving cat. I. During naturalistic behaviors and in response to simple and complex stimuli. Brain Res. 371:324.

363. Rehavi, M., O. Ramot, B. Yavetz, and M. Sokolovsky. (1980) Amitriptyline: long-term treatment elevates $\alpha$ adrenergic and muscarinic receptor binding in mouse brain. Brain Res. 194:443.

364. Richardson, B. P. and G. Engel. (1986) The pharmacology and function of 5-HT3 receptors. Trends Neurosci. september:424.

365. Riva, M. A. and I. Creese. (1989) Comparison of two putatively selective radioligands for labeling central nervous system $\beta$-adrenergic receptors: inadequacy of [3H]dihydroalprenolol. Mol. Pharmacol. 36:135.

366. Roberts, M. H. T. and D. W. Straughan. (1967) Excitation and depression of cortical neurones by 5hydroxytryptamine. J. Physiol. (Lond) 193:269.

367. Robinson, D. S., R. E. Gammans, R. C. Shrotriya, S. W. Jenkins, J. J. Andary, D. R. Alms, and M. E. Messina. (1990) Clinical effects of 5-HT 1 A partial agonists, buspirone and gepirone, in the treatment of depression. Clin. Neuropharmacol. 13 (suppl. 2):228.

368. Rogawski, M. A. and G. K. Aghajanian. (1981) Serotonin autoreceptors on dorsal raphe neurons: structureactivity relationships of tryptamine analogs. J. Neurosci. 1:1148.

369. Ropert, N. (1988) Inhibitory action of serotonin in $\mathrm{CA}_{1}$ hippocampal neurons in vitro. Neuroscience 26:69.

370. Ropert, N. and N. Guy. (1991) Serotonin facilitates GABAergic transmission in the $\mathrm{CA}_{1}$ region of rat hippocampus in vitro. J. Physiol. (Lond) 441:121.

371. Roth, B. L., M. Hamblin, and R. D. Ciaranello. (1990) Regulation of 5-HT2 and 5-HT IC serotonin receptor levels. Methodology and mechanisms. Neuropsychopharmacology 3:427.

372. Rowan, M. J. and R. Anwyl. (1985) The effect of prolonged treatment with tricyclic antidepressants on the actions of 5-hydroxytryptamine in the hippocampal slice of the rat. Neuropharmacology 24:131.

373. Rudorfer, M. V. and W.Z. Potter. (1989) Antidepressants: A comparative review of the clinical phamacology and therapeutic use of the newer versus the older drugs. Drugs. 37:713. 
374. Sakai, K., D. Salvert, M. Touret, and M. Jouvet. (1977) Afferent connections of the raphe dorsalis in the cat as visualized by the horseradish peroxidase technique. Brain Res. 137:11.

375. Sanders-Bush, E. and F. Sulser. (1986) Serotonin/norepinephrine receptor interactions: sensitivity changes after antidepressants and lesions. In Receptor-receptor interactions: $A$ new intramembrane integrative mechanism. K. Fuxe and L. F. Agnati, editors. Plenum Press, N.Y. and London. 387.

376. Sanghera, M. K., J. A. Coke, H. L. Williams, and B. A. McMillen. (1990) Ipsapirone and 1-(2-pyrimidinyl)piperazine increase rat locus coeruleus noradrenergic activity. Brain Res. Bull. 24:17.

377. Sanghera, M. K. and D. C. German. (1983) The effects of benzodiazepine and non-benzodiazepine anxiolytics on locus coeruleus unit activity. J. Neural Transm. 57:267.

378. Sapolsky, R. M. and P. M. Plotsky. (1990) Hypercortisolism and its possible neural bases. Biol. Psychiatry 27:937.

379. Sara, S. J. and O. Bergis. (1991) Enhancement of excitability and inhibitory processes in hippocampal dentate gyrus by noradrenaline: a pharmacological study in awake, freely moving rats. Neurosci. Lett. 126:1.

380. Savage, D. D., J. Mendels, and A. Frazer. (1980) Monoamine oxidase inhibitors and serotonin uptake inhibitors: Differential effects on [3H]serotonin binding sites in rat brain. J. Pharmacol. Exp. Ther. 168:297.

381. Schechter, L. E., F. J. Bolanos, H. Gozlan, L. Lanfumey, S. Haj-Dahmane, A. M. Laporte, C. M. Fattaccini, and M. Hamon. (1990) Alterations of central serotoninergic and dopaminergic neurotransmission in rats chronically treated with ipsapirone: biochemical and electrophysiological studies. J. Pharmacol. Exp. Ther. 255:1335.

382. Scheinin, M., J. W. Lomasney, D. M. Hayden-Hixson, U. B. Schambra, M. G. Caron, R. J. Lefkowitz, and R. T. Fremeau. (1994) Distribution of $\alpha_{2}$-adrenergic receptor subtype gene expression in rat brain. Mol. Brain Res. 21:133.

383. Schildkraut, J. J. (1965) The catecholamine hypothesis of affective disorders: A review of supporting evidence. Am. J. Psychiatry 122:509.

384. Schlicker, E., K. Fink, K. Classen, and M. Gothert. (1987) Facilitation of serotonin (5-HT) release in the rat brain cortex by cAMP and protable inhibition of adenylate cyclase in 5-HT nerve terminals by presynaptic $\alpha_{2}$ adrenoceptors. Naunyn-Schmiedeberg's Arch. Pharmacol. 336:251.

385. Schlicker, E., M. Gothert, and K. Hillenbrand. (1985) Cyanopindolol is a highly potent and selective antagonist at the presynaptic serotonin autoreceptor in the rat brain cortex. Naunyn-Schmiedeberg's Arch. Pharmacol. 331:398.

386. Schlicker, E., M. Gothert, F. Kostermann, and R. Clausing. (1983) Effects of $\alpha$-adrenoceptor antagonists on the release of serotonin and noradrenaline from rat brain cortex slices. Influence of noradrenaline uptake inhibition and determination of p A2 values. Naunyn-Schmiedeberg's Arch. Pharmacol. 323:106.

387. Schlicker, E., M. Guthert, F. Kostermann, and R. Clausing. (1983) Effects of $\alpha$-adrenoceptor antagonists on the release of serotonin and noradrenaline from rat brain cortex slices. Naunyn-Schmiedeberg's Arch. Pharmacol. 323:106.

388. Schoffelmeer, A. N. M. and A. H. Mulder. (1982) 3H-Noradrenaline and 3H-5-HT release from rat brain slices and its presynaptic $\alpha$-adrenergic modulation after long-term desipramine pretreatment. Naunyn-Schmiedeberg's Arch. Pharmacol. 318:173.

389. Schoffelmeer, A. N. M. and A. H. Mulder. (1983) 3H-Noradrenaline release from rat neocortical slices in the absence of extracellular calcium and its presynaptic $\alpha_{2}$-adrenergic modulation: a study on the possible role of cAMP. Naunyn-Schmiedeberg's Arch. Pharmacol. 323:188. 
390. Scott, J. A. and F. T. Crews. (1986) Down-regulation of seroioninz, but not of $\beta$-adrenergic receptors during chronic treatment with amitriptyline is independent of stimulation of serotonin2 and $\beta$-adrenergic receptors. Neuropharmacology 25:1301.

391. Scuvée Moreau, J.. I. Giesbers. and A. Dresse. (1987) Electrophysiological and microiontophoretic studies with buspirone: influence on the firing rate of central monoaminergic neurons and their responsiveness to dopamine. clonidine or GABA. Arch. Int. Physiol. Biochim. 95:439.

392. Scuvée-Moreau, J. J. and A. Dresse. (1979) Effect of various antidepressant drugs on the spontancous firing rate uf locus coenuleus and dorsal raphe neurons of the rat. Eur. J. Pharnuacol. 57:219.

393. Scuvee-Moreau, J. J. and T. H. Svensson. (1982) Sensitivity in vivo of central $\alpha_{2}$ - and opiate receptors after chronic treatment with various antidepressants. J. Neural Transm. 54:51.

394. Segal, M. (1979) Serotonergic innervation of the locus coeruleus from the dorsal raphe and its action on responses to noxious stimuli. J. Physiol. (Lond) 286:401.

395. Segal, M. (1980) The action of scrotonin in the rat hippocampal slice preparation. J. Physiol. (Lond) 303:423.

396. Segal, M. (1982) Norepinephrine modulates reactivity of hippocampal cells to chemical stimulation in vitro. Exp. Neurol. 77:86.

397. Segal, M. and F. E. Bloom. (1974) The action of norepinephrine in the rat hippocampus. Il. Activation of the input pathway. Brain Res. 72:99.

398. Segal, M. and F. E. Bloom. (1976) The action of norepinephrine in the rat hippocampus. IV. The effects of locus coeruleus stimulation on evoked hippocampal unit activity. Brain Res. 107:513.

399. Segal, M., H. Markram, and G. Richter-Levin. (1991) Actions of norepinephrine in the rat hippocampus. Prog. Brain Res. 88:323.

400. Seligman, M. E. P. (1975) Helplesness: On depression, development and death. Freeman, San Francisco.

401. Sharp, T. and S. Hjorth. (1990) Application of brain microdialysis to study the pharmacology of the 5-HT IA autoreceptor. J. Neurosci. Methods 34:83.

402. Shenker, A., S. Maayani, H. Weinstein, and J. P. Green. (1987) Phamacological characterization of two 5hydroxytryptamine receptors coupled to adenylate cyclase in guinea pig hippocampal membranes. Mol. Pharmacol. 31:357.

403. Sherman, A. D. and F. Petty. (1980) Neurochemical basis of the action of antidepressants on learned helplessness. Behav. Neural Biol. 30:119.

404. Shopsin, B., G. B. Cassano, and L. Conti. (1981) An overview of new "second generation" antidepressant compounds: Research and treatment implications. In Antidepressants: neurochemical, behavioral, and clinical perspectives. S. J. Enna J.B. Malick and E. Richelson editors. Raven, N.Y.. 219.

405. Shopsin, B., E. Friedman, and S. Gershon. (1976) Parachlorophenylalanine reversal of tranylcypromine effects in depressed patients. Arch. Gen. Psychiatry 33:811.

406. Shopsin, B., S. Gershon, and M. Goldstein. (1975) The use of synthesis inhibitors in defining a role for biogenic amines during imipramine treatment in depressed patients. Psychopharmacol. Commun. 1:239.

407. Siever, L. J. and K. L. Davis. (1985) Overview: toward a dysregulation hypothesis of depression. Am. J. Psychictry 142:1017. 
408. Sladek, J. and P. Walker. (1977) Serotonin-containing neuronal perikarya in the primate locus coeruleus and subcocruleus nuclei. Brain Res. 134:359.

409. Sleight, A. J., C. A. Marsden, M. G. Palfreyman, A. K. Mir, and W. Lovenberg. (1988) Chronic MAO A and MAO B inhibition decreases the 5-HT $\mathrm{H}_{1 \mathrm{~A}}$ receptor-mediated inhibition of forskolin-stimulated adenylate cyclase. Eur. J. Pharmacol. 154:255.

410. Smil', C. B., J. A. Garcia-Sevilla, and P. J. Hollingsworth. (1981) $\alpha_{2}$-Adrenoceptors in rat brain are decreased after long-term tricyclic antidepressant drug treatment. Brain Res. 210:413.

411. Soubrie, P., P. Martin, S. el Mestikawy, and M. Hamon. (1987) Delayed behavioral response to antidepressant drugs following sclective damage to the hippocampal noradrenergic innervation in rats. Brain Res. 437:323.

412. Sprouse, J. S. and G. K. Aghajanian. (1986) (-)-Propanolol blocks the inhibition of serotonergic dorsal raphe cell firing by $5-\mathrm{HT}_{1 \mathrm{~A}}$ agonists. Eur. J. Pharmacol. 128:295.

413. Sprouse, J. S. and G. K. Aghajanian. (1987) Electrophysiological responses of serotoninergic dorsal raphe neurons to 5-HT $1 \mathrm{~A}$ and 5-HT $1 \mathrm{~B}$ agonists. Synapse 1:3.

414. Stanford. C., D. J. Nutt, and P. J. Cowen. (1983) Comparison of the effects of chronic desmethylimipramine administration on $\alpha_{2}$ - and $\beta$-adrenoceptors in different regions of rat brain. Neuroscience 8:161.

415. Stanford, S. C. and D. J. Nutt. (1982) Comparison of the effects of repeated electroconvulsive shock on $\alpha_{2-}$ and (j-adrenoceptors in different regions of rat brain. Neuroscience 7:1753.

416. Stanford, S. C., D. J. Nutt, and P. J. Cowen. (1983) Comparison of the effects chronic desmethylimipramine administration on $\alpha_{2}$ and beta-adrenoceptors in different regions of rat brain. Neuroscience 8:161.

417. Stanton, P. K. and U. Heinemann. (1986) Norepinephrine enhances stimulus-evoked caicium and potassium concentration changes in dentate granule cell layer. Neurosci. Lett. 67:233.

418. Stanton, P. K., I. Mody, and U. Heinemann. (1989) A role for N-methyl-D-aspartate receptors in norepinephrineinduced long-lasting potentiation in the dentate gyrus. Exp. Brain Res. 77:517.

419. Stanton, P. K. and J. M. Sarvey. (1985) The effect of high-frequency electrical stimulation and norepinephrine on cyclic AMP levels in normal versus norepinephrine-depleted rat hippocampal slices. Brain Res. 358:343.

420. Stanton, P. K. and J. M. Sarvey. (1985) Blockade of norepinephrine-induced long-lasting potentiation in the hippocampal dentate gyrus by an inhibitor of protein synthesis. Brain Res. 361:276.

421. Stanton, F. K. and J. M. Sarvey. (1987) Norepinephrine regulates long-term potentiation of both the population spike and dendritic EPSP in hippocampal dentate gyrus. Brain Res. Bull. 18:115.

422. Starke, K. (1987) Presynaptic $\alpha$-autoreceptors. Rev. Physiol. Biochem. Pharmacol. 107:73.

423. Starke, K., M. Gothert, and H. Kilbinger. (1989) Modulation of neurotransmitter release by presynaptic autoreceptors. Physiol. Rev. 69:864.

424. Starke, K. and H. Montel. (1973) Involvement of $\alpha$-receptors in clonidine induced inhibition of transmitter release from central morioamine neurones. Neuropharmacology 12:1073.

425. Stockmeier, C. A., A. M. Martino, and K. J. Kellar. (1985) A strong influence of serotonin axons on $\beta$-adrenergic receptors in rat brain. Science 230:323.

426. Stockneier, C. A., S. W. McLeskey, J. A. Blendy, N. R. Amstrong, and K. J. Kellar. (1987) Electroconvulsive shock but not antidepressant drugs increases $\alpha_{1}$-adrenoceptor binding sites in rat brain. Eur. J. Pharmacol. 139:259. 
427. Suaud-Chagny, M. F., C. Mermet. E. Tibiriça, P. Bousquet, and F. Gonon. (1992) Does rilmenidine act in vivo on central $\alpha_{2}$-adrenoceptors modulating noradrenaline release. Eur. J. Phormacol. 213:305.

428. Sulser, F., A. J. Janowsky, F. Okada, D. H. Manier, and P. L. Mobley. (1983) Regulation of recognition and action function of the norepinephrine (NE) receptor-coupled adenylate cyclase system in brain: implications for the therapy of depression. Neuropharmacology 22:425.

429. Sulser, F. and E. Sanders Bush. (1987) The serotonin-norepinephrine link hypothesis of affective disorders: receptor-receptor interactions in brain. Adv. Exp. Med. Biol. 221:489.

430. Svensson, T. H. (1980) Effect of chronic treatment with tricyclic antidepressant drugs on identified brain noradrenergic and serotonergic neurons. Acta Psychiatr. Scand. 61:121.

431. Svensson, T. H., B. S. Bunney, and G. K. Aghajanian. (1975) Inhibition of both NA and 5-HT neurons in brain by the $\alpha$-adrenergic agonist clonidine. Brain Res. 92:291.

432. Svensson, T. H. and T. Usdin. (1978) Feedback Inhibition of brain noradrenaline neurons by tricyclic antidepressants: alpha receptor mediation. Science 202:1089.

433. Tepper, J. M., S. Nakamura, C. W. Spanis, L. R. Squire, S. J. Young, and P. M. Groves. (1982) Subsensitivity of catecholaminergic neurons to direct acting agonists after single or repeated electroconvulsive shock. Biol. Psychiatry 17:1059.

434. Thomas, D. N., D. J. Nutt, and R. B. Holman. (1992) Effects of acute and chronic electroconvulsive shock on noradrenaline release in the rat hippocampus and frontal cortex. Br. J. Pharmacol. 106:430.

435. Tibirica, E., J. Feldman, C. Mermet, F. Gonon, and P. Bousquet. (1991) An imidazoline-specific mechanism for the hypotensive effect of clonidine: a study with yohimbine and idazoxan. J. Pharmacol. Exp. Ther. 256:606.

436. Tiong, A. H. and J. S. Richardson. (1990) The characterization of $\beta$-adrenoceptor subtypes in the rat amygdala and hippocampus. Int. J. Neurosci. 54:231.

437. Tork, I. (1990) Anatomy of the serotonergic system. Ann. N. Y. Acıd. Sci.600:9.

438. Trendelenburg, A. -U., M. Trendelenburg, K. Starke, and N. Limberger. (1994) Release-inhibiting $\alpha_{2-}$ adrenoceptors at serotonergic axons in rat and rabbit cortex: evidence for pharmacological identity with $\alpha_{2}$ autoreceptors. Naunyn-Schmiedeberg's Arch. Pharmacol. 349:25.

439. Trulson, M. E. and T. Crisp. (1984) Role of norepinephrine in regulating the activity of serotonin-containing dorsal raphe neurons. Life Sci. 35:511.

440. Trulson, M. E. and B. L. Jacobs. (1979) Raphe unit activity in freely moving cats: correlation with level of behavioral arousal. Brain Res. 163:135.

441. U'Prichard, D. C., W. D. Bechtel, B. M. Rouot, and S. H. Snyder. (1979) Multiple apparent $\alpha$-noradrenergic receptor binding sites in rat brain: Effect of 6-OHDA. Mol. Pharmacol. 16:47.

442. Uhien, S., Y. Xia, V. Chhajlani, C. C. Felder, and J. E. S. Wikberg. (1992) [3H]-MK 912 binding delineates two $\alpha_{2}$-adrenoceptor subtypes in rat CNS one of which is idertical with the cloned p A2d $\alpha_{2}$-adrenoceptor. Br. J. Pharmacol. 106:986.

443. Innerstall, J. R., I. Femandez, and L. M. Orensanz. (1985) The alpha-adrenergic receptor: radiohistochemical analysis of functional characteristics and biochemical differences. Pharmacol. Biochem. Behav. 22:859.

444. Valentino, R. J., A. L. Curtis, D. G. Parris, and R. G. Wehby. (1990) Antidepressant actions on brain noradrenergic neurons. J. Pharmacol. Exp. Ther. 253:833. 
445. van Praag, H. M. (1983) In search of the mode of action of antidepressants: 5-HTP/tyrosine mixtures in depressions. Neuropharmacology 22:433.

446. VanderMaelén, C. P. and G. K. Aghajanian. (1983) Electrophysiclogical and pharmacological characterization of serotonergic dorsal raphe neurons recorded extracellularly and intracellularly in rat brain slices. Brain Res. 289:109.

447. VanderMaelen, C. P. and J. P. Braselton. (1992) Effects of the antidepressant compound nefazodone sn central monoaminergic neuronal discharge in rats. Drug Dev. Res. 25:235.

448. VanderMaelen, C. P., G. K. Matheson, R. C. Wilderman, and L. A. Patuerson. (1986) Inhibition of serotonergic dorsal raphe neurons by systemic and iontophoretic administration of buspirone, a non-benzodiazepine anxiolytic drug. Eur. J. Pharmacol. 129:123.

449. Varrault, A., V. Leviel, and J. Bockaert. (1991) S-HT1A -sensitive adenylyl cyclase of rodent hippocampal neurons: effects of antidepressant treatments and chronic stimulation with agonists. J. Pharmacol. Exp. Ther. 257:433.

450. Verge, D., G. Daval, M. Marcinkiewicz, A. Patey, S. el Mestikawy, H. Gozlan, and M. Hamon. (1986) Quantitative autozadiography of multiple 5-HT 1 receptor subtypes in the brain of control or 5,7-dihydroxytryptamine-treated rats. J. Neurosci. 6:3474.

451. Verge, D., G. Daval, A. Patey, H. Gozlan, S. el Mestikawy, and M. Hamon. (1985) Presynaptic 5-HT autoreceptors on serotonergic cell bodies and/or dendrites but not terminals are of the 5-HT 1 A subtype. Eur.J. Pharmacol. 113:463.

452. Vetulini, J., L. Antkiewicz-Michaluk, and A. Rokosz-Pelc. (1984) Chronic administration of antidepressant drugs incresses the density of cortical 3H-prazosin binding sites in the rat brain. Brain Res. 310:360.

453. Vetultuni, J., R. J. Stawarz, and F. Sulser. (1976) Adaptative mechanisms of the noradrenergic cyclic AMP gencrating system in the limbic forebrain of the rat: Adaptation to persistent changes in the availability of norepinephrine (NE). J. Neurochem. 27:661.

454. Villani, F. and D. Johnston. (1993) Serotonin inhibits induction of long-term potentiation at commissural synapses in hippocampus. Brain Res. 606:304.

455. Vizi, E. S., I. J. Elenkov, F. Oberfrank, J. Kiss, and L. G. Harsing. (1992) Role of presynaptic $\alpha_{2}$-heteroreceptors in nonsynaptic modulation of transmitter release. Ad. Bioscience 82:297.

456. Wang, H. Y. and E. Friedman. (1988) Chronic lithium: desensitization of autoreceptors mediating serotonin release. Psychopharmacology 94:312.

457. Wang, R. Y. and G. K. Aghajanian. (1977) Antidromically identified serotonergic neurons in the rat midbrain raphe: Evidence for collateral inhibition. Brain Res. 132:186.

458. Wang, R. Y. and G. K. Aghajanian. (1980) Enhanced sensitivity of amygdaloid neurons to serotonin and norepinephrine after chronic antidepressant treatment. Commun. Psychopharmacol. 4:83.

459. Wang, Y. Y. and G. K. Aghajanian. (1987) Excitation of locus coeruleus neurons by an adenosine 3',5'-cyclic monophosphate-activated inward current: extracellular and intracellular studies in rat brain slices. Synapse 1:481.

460. Watanabe, Y., R. R. Sakai, B. S. McEwen, and S. Mendelson. (1993) Stress and antidepressant effects on hippocampal and cortical 5-HT, $1 \mathrm{~A}$ and 5-HT2 receptors and transport sites for serotonin. Brain Res. 615:87.

461. Weiner, N. and P. B. Molinoff. (1994) Catecholamines. In Basic Neurochemistry: Molecular, cellular, and medical aspects. G. J. Siegel, B. W. Agranoff, R. W. Albers, and P. B. Molinoff, editors. Raven, New York. 261-281.

462. Weissmann-Nanopoulos, D., E. Mach, J. Magre, Y. Demassey, and J. F. Pujol. (1985) Evidence for the localization of 5-HT $1 \mathrm{~A}$ binding sites on serotoniti containing neurons in $\mathrm{t}$.. " phe dorsalis and raphe centralis nuclei of the rat brain. Neurochem. Int. 7:1061. 
463. Welner, S. A., C. de Montigny, J. Desroches, P. Desjardins, and B. E. Suranyi-Cadotte. (1989) Autoradiographic quantification of serotonin 1 A receptors in rat brain following antidepressant drug treatment. Synopse 4:347.

464. Westlund, K. N., R. M. Denney, R. M. Rose, and C. W. Abell. (1988) Localization of distinct monoamine oxidase A and monoamine oxidase B cell populations in human brainstem. Neuroscience 25:439.

465. Wilkinson. L. O. and B. L. Jacobs. (1988) Lack of response of serotonergic neurons in the dorsal raphe nucleus of freely moving cats to stressful stimuli. Exp. Neurol. 101:445.

466. Williams, J. T. (1988) Voltage- and ligand-activated inwardly rectifying currents in dorsal raphe neurons in vitro. J. Neurosci. 8(9):3499.

467. Williams, J. T., G. Hendersen, and R. A. North. (1985) Characterization of $\alpha_{2}$-adrenoceptors which increase potassium conductance in rat locus coeruleus neurones. Neuroscience 14:95.

468. Williams, J. T. and R. A. North. (1985) Catecholamine inhibition of calcium action potentials in rat locus coeruleus neurones. Neuroscience 14:103.

469. Willner, P. (1985) Noradrenaline and depression: Theoritical perspectives. In Depression: A psychobiological synthesis. Wiley, New York. 193-215.

470. Willner, P. (1985) Depression: A psychobiological synthesis. Wiley, New York.

471. Willner, P. (1985) Antidepressants and serotonin. In Depression: A Psychobiological synthesis. Wiley, New York. 327-351.

472. Winson, J. and D. Dahl. (1985) Action of norepinephrine in the dentate gyrus. II. Iontophoretic studies. Exp. Brain Res. 59:497.

473. Wolfe, D. B., T. K. Harden, J. R. Sporn, and P. B. Molinoff. (1978) Presynaptic modulation of $\beta$-adrenergic receptors in rat cerebral cortex after treatment with antidepressants. J. Pharmacol. Exp. Ther. 207:305.

474. Yakel, J. L. (1992) 5-HT3 receptors as cation channels. In Central and peripheral 5-HT3 receptors. Academic Press, 102-127.

475. Yakel, J. L. and M. B. Jackson. (1988) 5-HT 3 receptors mediate rapid responses in cuitured hippocampus and a clonal cell line. Neuron. 1:615.

476. Yamamoto, H., U. Tomita, M. Mikuni, I. Kobayashi, A. Kagaya, T. Katada, M. Ui, and K. Takahashi. (1992) Direct activation of purified $G_{0}$-type GTP binding protein by tricyclic antidepressants. Neurosci. Lett. 139:194.

477. Yazici, O., F. Aricioglu, G. Gürvit, A. Uçok, Y. Tastaban, . Canberk, M. Ozgüroglu, T. Durat, and D. Sahin. (1993) Noradrenergic and serotoninergic depression. J. Affect. Disord. 27:123.

478. Young, E. A., S. Akana, and M. F. Dallman. (1990) Decreased sensitivity to glucocorticoid fast feedback in chronically stressed rats. Neuroendocrinology 51:536.

479. Young, E. A., R. F. Haskett, V. Murphy-Weinberg, S. J. Watson, and Y... Akil. (1991) Loss of glucocorticoid fast feedback in depression. Arch. Gen. Psychiatry 48:693.

480. Zemlan, F. P. and D. L. Garver. (1990) Depression and antidepressant therapy: receptor dynamics. Prog. Neuropsychopharmacol. Biol. Psychiatry 14:503.

481. Zgombick, J. M., S. G. Beck, C. D. Mahle, B. Craddock-Royal, and S. Maayani. (1989) Pertussis toxin-sensitive guanine nucleotide-binding protein(s) couple adenosine Al and 5-hydroxytryptamine ${ }_{1 \mathrm{~A}}$ receptors to the same cffector systems in rat hippocampus: biochemical and electrophysiological studies. Mol. Pharmocol. 35:484. 
First article

This research endeavour had essentially two aims: first, to identify and characterize adrenergic and 5-HT receptors implicated in interactions between the NA and 5-HT systems of the hippocampus, and second, to determine whether these interactions are altered by antidepressant treatments. The presence of $\alpha_{2}$-adrenergic heteroreceptors on 5-HT fibers was well documented in the early 80's in in vitro studies using brain slices. However, the in vivo electrophysiological characterization of these adrenoceptors had not been done. In this first study, we performed this task using the technique of electrical stimulation of the ascending 5-HT pathway coupled with orthodromic recording in the $\mathrm{CA} 3$ region of the rat hippocampus. In brief, stimulation of the 5-HT pathway produces a suppression of firing of pyramidal neurons, the duration of which is determined by the sensitivity of postsynaptic 5-HT1A receptors and by the amount of 5-HT released into the synaptic cleft. For instance, agents that decrease the release of 5-HT from terminals by activating 5-HT terminal autoreceptors have been shown to decrease the duration of suppression of firing. This electrophysiological paradigm allowed us to resolve an important issue, that is, the presence or the absence in vivo of a tonic inhibitory action of endogenous NA on $\alpha_{2}$-adrenoceptors of the 5-HT fibers.

This article entitled "In vivo electrophysiological evidence for a tonic activation by endogenous noradrenaline of $\alpha_{2}$-adrenoceptors on 5-hydroxytryptamine terminals in the rat hippocampus" by myself, Pierre Blier and Claude de Montigny was published in the Naunyn-Schmiedeberg's Archives of Pharmacology (1993, vol. 347, pp. 266-272). 


\title{
In vivo electrophysiological evidence for tonic activation by endogenous noradrenaline of $\alpha_{2}$-adrenoceptors on 5-hydroxytryptamine terminals in the rat hippocampus
}

\author{
Raymond Mongeau, Pierre Blier, and Claude de Montigny \\ Neurobiological Psychiatry Unit, Department of Psychiatry, McGill University, 1033 Pine Avenue West, Montrtal, Quebec, Canada H3A IAI \\ Received August 11, 1992/Accepted November 27, 1992
}

Summary. The activation of $\alpha_{2}$-adrenergic heteroreceptors was studied by comparing the effectiveness of the electrical stimulation of the ascending 5-HT pathway in suppressing the firing activity of $\mathrm{CA}_{3}$ dorsal hippocampus pyramidal neurons prior to, and following, the intravenous administration of noradrenergic agents. Desipramine ( $2 \mathrm{mg} / \mathrm{kg})$, a selective noradrenaline reuptake blocker, reduced the efficacy of the stimulation; this effect was reversed by the $\alpha_{2}$-adrenoceptor antagonists yohimbine $(0.5 \mathrm{mg} / \mathrm{kg})$ and $(-)$ mianserin $(0.5 \mathrm{mg} / \mathrm{kg})$, but not by idazoxan $(0.5 \mathrm{mg} / \mathrm{kg})$, an adrenoceptor antagonist with preferential affinity for the imidazoline recognition sites. Low doses of the $\alpha_{2}$-adrenoceptor agonist clonidine (2 and $10 \mu \mathrm{g} / \mathrm{kg}$ ) enhanced the efficacy of the stimulation, while high doses $(100$ and $400 \mu \mathrm{g} / \mathrm{kg})$ reduced it. These incremental and decremental effects of clonidine were reversed by 0.1 and $1 \mathrm{mg} / \mathrm{kg}$ of yohimbine, respectively. The enhancing ffect of the low dose of clonidine $(10 \mu \mathrm{g} / \mathrm{kg})$ was abolished in rats pretreated with the noradrenaline neurotoxin 6-hydroxydopamine. However, the inhibitory effect of a high dose of clonidine $(100 \mu \mathrm{g} / \mathrm{kg})$ was unaltered by this pretreatment. These results indicate that low doses of clonidine preferentially activate $\alpha_{2}$. adrenergic autoreceptors on the noradrenaline neurons resulting in a reduction of the tonic inhibitory effect of endogenous noradrenaline on 5-HT neurotransmission, while higher doses of clonidine would decrease 5-HT neurotransmission through the direct activation of $\alpha_{2}$-adrenergic heteroreceptors on 5-HT terminals. Furthermore, the selective $\alpha_{2}$-adrenergic heteroreceptors antagonist (-)mianserin $(0.5 \mathrm{mg} / \mathrm{kg})$ increased by itself the efficacy of 5.HT neurotransmission, an effect not observed with yohisibine and idazoxan. Taken together, these results suggest that, in vivo, the $\alpha_{2}$-adrenoceptors on 5-HT terminals of the rat hippocampus are tonically activated by endogenous noradrenaline and modulate 5-HT release.

Correspondence 10 R. Mongeau at the above address
Key words: $a_{2}$-Adrenergic heteroreceptors - Electrophysiology - Hippocampus - Presynaptic modulation - Serotonin

\section{Introduction}

Several in vitro studies, using brain slices or synapto. somes, have provided evidence for the existence of $a_{2}$-adrenoceptors on 5-HT terminals in the brain of different species (for a review see Gothert and Schlicker 1991, see also: Raiteri et al. 1990; Gobbi et al. 1990; Maura et al. 1992). The data on the tonic inhibitory action of endoge. nous noradrenaline on $\alpha_{2}$-adrenoceptors modulating the in vitro release of $\left.{ }^{3} \mathrm{H}\right] 5-\mathrm{HT}$ from prelnaded brain slices are, however, conflicting. On the one hand, experiments with classical $\alpha_{2}$-adrenoceptor antagonists have generally suggested that $\alpha_{2}$-adrenergic heteroreceptors are not tonically activated by endogenous noradrenaline (Gothert et al. 1981; Schlicker et al. 1983; Galzin et al. 1984). On the other hand, the enhanced evoked release of $\left[{ }^{3} \mathrm{H}\right] \mathrm{S}-\mathrm{HT}$ following exposure to high concentrations of the $\alpha$-adrenoceptor antagonist phentolamine has been interpreted as an evidence for the presence of a tonic inhibitory action of endogenous noradrenaline on S.HT neurotransmission (Gothert and Huth 1980; Feuerstein et al. 1985; Frankhuijzen et al. 1988). However, further studies by Limberger et al. (1989) have shown that phentolansine enhances $\left[{ }^{3} \mathrm{H}\right]$ 5-HT release, not by its action at $\alpha_{2}$-adisnergic heteroreceptors, but rather by blocking 5-HT autoreceptors.

Using rabbit hippocampus slices, Feuerstein et al. (1985) have reported that endogenous noradrenaline can activate $a_{2}$-adrenergic heteroreceptors when its synaptic concentration is increased by blocking the noradrenaline reuptake carrier. This phenomenon was not observed in studies using preloaded slices of rat hypothalamus (Galzin et al. 1984), rat hippocampus (Benkirane et al. 1985) and rabbit cerebral cortex (Limberger et al. 1986). Taken together, these results obtained in vitro suggest that the aciivation of $a_{2}$-adrenoceptors on 5-HT terminals by 
endogenous noradrenaline is rather weak, if not absent, depending on the species and/or the brain structure. However, the activation of these $\alpha_{2}$-adrenergic heteroreceptors by endogenous noradrenaline appears to be functionally important in vivo since the responsiveness of these receptors to agonist stimulation was increased following noradrenergic dinervation with DSP4 (Benkirane et al. 1985; Ellison and Campbell 1986). Therefore, studies using int vitro preparations may not be adequate to investigate the inhibitory action of endogenous noradrenaline on 5 -HT release. Consequently, it was deemed crucial to assess the function of the $\alpha_{2}$-adrenoceptors that modulate $5-\mathrm{HT}$ neurotransmission using an in vivo electrophysiological paradigm.

Three strategies were used in the present study to investigate the function of $a_{2}$-adrenergic hetero- and autoreceptors in modulating 5-HT release. First, the concentration of synaptic noradrenaline was increased by the acute blockade of the noradrenergic reuptake carrier with desipramine. Second, different doses of the $\alpha_{2}$-adrenoceptor agonist clonidine were tested on the efficacy of the stimulation of the 5-HT pathway. Third, the effects of the $\alpha_{2}$-adrenoceptor antagonists yohimbine, idazoxan or $(-)$ mianscrin were also assessed on their own and following the administration of clonidine or desipramine. Yohimbine is a classical $a_{2}$-adrenoceptor antagonist (Goldberg and Robertson 1983), whereas idazoxan is an $\alpha_{2}$-adrenoceptor antagonist with affinity for the imidazoline recognition site (Hamilton et al. 1988; Michel et al. 1990). The $(-)$ stereoisomer of mianserin is a particularly interesting compound since it has been reported to block the action of noradrenaline at $a_{2}$-adrenergic heteroreceptors on 5-HT terminals, but not at $\alpha_{2}$-adrenergic autoreceptors on noradrenergic terminals (Raiteri et al. 1083; Maura et al. 1985).

\section{Materials and methods}

Male Sprague-Dawley rats $(225-300 \mathrm{~g})$ maintained on a $12: 12$ lightdask cycle with free access to food and water were used. Lesions of noradrenaline neurons were performed on a group of eight rats that received, under chloral hydrate anesthesia $(400 \mathrm{mg} / \mathrm{kg}$. i. p.), an intracerebroventricular injection of 6-hydroxydopamine (6-OHDA; $120 \mu \mathrm{g}$ free base in $20 \mu \mathrm{l}$ of $0.9 \% \mathrm{NaCl}$ and $0.1 \%$ ascorbie acid), $1 \mathrm{~h}$ af. ter an injection of fluoxetine $(10 \mathrm{mg} / \mathrm{kg}, i, p$.) administered to protect the s.HT system from the neurotoxic action of 6-OHDA. A control group of four sats were injected with $20 \mu \mathrm{l}$ of $0.9 \% \mathrm{NaCl}$.

Recording from dorso' nippocampus $\mathrm{CA}_{3}$ pyramidal neurons. Extracellular unitary; iscordings and microiontophoresis were performed in the $\mathrm{CA}_{3}$ layer of hippocampus with five-barrelled micropipettes, pulled in a conventional manner with the tip broken to a diameter of 9-11 $\mu \mathrm{m}$. The central barrel, used for recording, was filled with a $2 \mathrm{~mol} / \mathrm{l} \mathrm{NaCl}$ solution and the side barrels contsined the following solutions: S-HT creatinine sulfate $(0.5 \mathrm{mmol} / /$ in $200 \mathrm{mmol} / \mathrm{l} \mathrm{NaCl}, \mathrm{pH} 4)$, noradrenaline bitartrate $(20 \mathrm{mmol} / 1$ in $200 \mathrm{mmol} / / \mathrm{NaCl}$, pH 4), acetylcholine chloride $(20 \mathrm{mmol} / 1$ in $200 \mathrm{mmol} / \mathrm{l} \mathrm{NaCl}, \mathrm{pH} 4)$ and a $2 \mathrm{~mol} / 1 \mathrm{NaCl}$ solution used for automatic current balancing. The 5.HT and noradrenaline solutions were retained with a $-7 \mathrm{nA}$ current be. tween ejections. Pyramidal neurons were identified by their large amplitude $(0.5-1.2 \mathrm{mV})$ and long duration $(0.8-1.2 \mathrm{~ms})$ simple spikes alternating with complex spike discharges (Kandel and Spencer 1961). These characteristics readily permit to differentiate pyramidal neurons from interneurons. A leak or small current of acetylcholine $(-1$ to $5 \mathrm{nA})$ was used to activate silent or slowly discharging pyramidal neurons to a physiologic firing rate $(8-12 \mathrm{~Hz})$, since most of these cells do not discharge spontaneously in chloral hydrate-anesthetized rats.

To evaluate the effectiveness of the 6-OHDA lesion, the recovery of the firing rate of the pyramidal neurons following the microiontophoretic applisation of noradrenaline was assessed by determining the recovery time $\mathbf{S O}\left(\mathrm{RT}_{90}\right)$. This parameter is defined by the time (in seconds) required for the firing rate to recover by $50 \%$ from the termination of the microiontophoretic application (de Montigny et al. 1980).

Stimulation of the S-HT pathway. To activate the S-HT projections to the dorsal hippocampus, a bipolar electrode (NE-100; David Kopf, Tujunga. Calif., USA) was implanted on the midline with a $10^{\circ}$ backward angle in the ventromedial tegmentum, $1 \mathrm{~mm}$ anterior to lamda and $8.3 \mathrm{~mm}$ below the cortical surface. Two hundred square pulses of $0.5 \mathrm{~ms}$ were delivered by a stimulator (\$8800; Grass Instruments, Quincey, Mass., USA) at a frequency of $1 \mathrm{~Hz}$ and at an intensity of $300 \mu \mathrm{A}$. The stimulation pulses and the firing activity of the neuron recorded were fed 10 an IBM-PC computer equipped with a Tecmar interface. Peristimulus time histograms were generated to determine the duration of suppression of firing, measured in absolute silence value (SIL, in ms) (Chaput et al. 1986). This parameter is obtained by dividing the total number of events suppressed following the stimulation by the mean frequency of firing of the neuron recorded. It thus represents an estimation of the duration of the suppression of firing corrected for the mcan prestimulation firing rate of the neuron recorded. The effect of stimulating the ascending 5-HT fibers was determined for the same neuron prior to, and following, the intravenous injection of desipramine $(2 \mathrm{mg} / \mathrm{kg})$, clonidine $(2-400 \mu \mathrm{g} / \mathrm{kg})$, yohimbine $(0.1-1 \mathrm{mg} / \mathrm{kg})$, (-)mianserin $(0.5 \mathrm{mg} / \mathrm{kg})$ or idazoxan $(0.5 \mathrm{mg} / \mathrm{kg})$. The time elapsed between the control stimulation periods and those following the administration of the drugs was generally around $15 \mathrm{~min}$.

Drugs. The following drugs were used: desipramine (Merrell Dow, Cincinnati, $\mathrm{OH}$, USA), (-)mianserin (Organon, Oss, The Netherlands), yohimbine $\mathbf{H C l}$, idazoxan $\mathrm{HCl}$, clonidine $\mathrm{HCl}, 5-\mathrm{HT}$ creatinine sulfate, noradrenaline bitartrate, 6-OHDA $\mathrm{HCl}$ and acetylcholine chloride (Sigma Chemicals, St. Louis, Mo., USA) and chloral hydrate (American Chemicals, Montréal, Québec, Canada).

Statistical analysis. All results are expressed as means \pm SEM. The statistical significance of the difference between the effects of the stimulation of the S.HT parbway prior to, and following, the administration of a drug was assessed with the two-tailed paired Student's t-test. The degree of statistical significince of the difference between the $R T_{s 0}$ of the intact rats and the 6-OHDA-treated rats was calculated with the twotailed Student's i-test.

\section{Results}

\section{Effect of acute desipramine administration on the efficacy of 5-HT neurotransmission}

Desipramine $(2 \mathrm{mg} / \mathrm{kg})$, a specific noradrenaline reuptake inhibitor (Ross and Renyi 1975), was tested in order to examine the effect of an increased synaptic availability of endogenous noradrenaline on the efficacy of the stimulation of the 5-HT pathway to suppress the firing activity of dorsal hippocampus $\mathrm{CA}_{3}$ pyramidal neurons. As shown in Fig. 1 A, the administration of desipramine markedly reduced the duration of suppression of firing. The SIL value after desipramine administration was $48 \%$ smaller than that before the injection of the drug (Fig. 1 B).

In order to confirm the $a_{2}$-adrenergic nature of this effect of desipramine, the $a_{2}$-adrenoceptor antagonists yohimbine idazoxan or $(-)$ mianserin were injected following the administration of desipramine. Yohimbine 
A

PRIOR TO

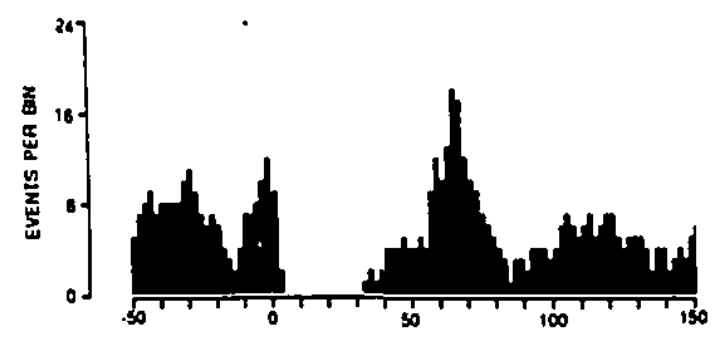

FOLLOWING DESIPRAMINE (2 mg/kg)

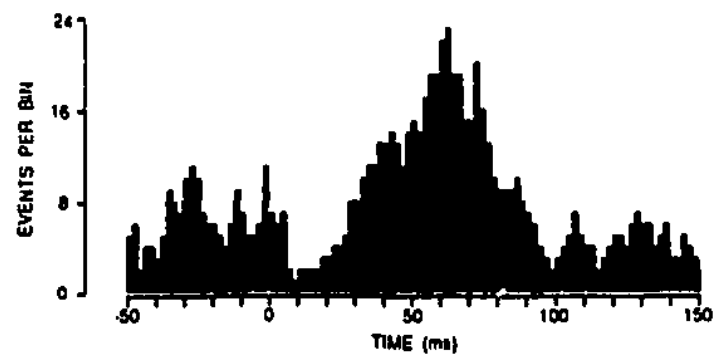

$(0.5 \mathrm{mg} / \mathrm{kg})$, a classical $\alpha_{2}$-adrenoceptor antagonist, reversed the effect of desipramine (Fig. 2 A), while idazoxan $(0.5 \mathrm{mg} / \mathrm{kg})$, an adrenoceptor antagonist with affinity for the imidazoline recognition site, did not alter significantly the effect of desipramine (Fig. 2B). The $\alpha_{2}$-adrenergic heteroreceptor antagonist (-)miariserin $(0.5 \mathrm{mg} / \mathrm{kg}) \mathrm{re}$ versed the suppressant effect of desipramine (Fig. 2C).

\section{Effect of acute clonidine administration on the efficacy 5.HT neurotransmission}

The dose-response curve depicted in Fig. 3 shows that clonidine exerted a biphasic effect on S-HT neurotransmi.ssion. Low doses of clonidine (2 and $10 \mu \mathrm{g} / \mathrm{kg}$ ) en-
B

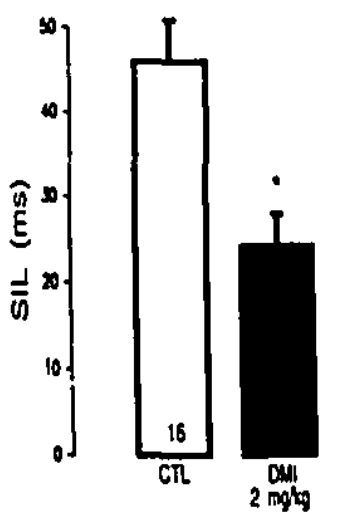

Fig. 1A,B. Effect of intravenous injections of desipramine on the efficacy of the electrical stimulation of the ascending S-HT path. way in suppressing the firing activity of $\mathrm{CA}_{\mathbf{3}}$ pyramidal neurons of hippocampus. A Representative peristimulus time histograms illustrating the effect of desipramine (DMI) in a single experiment. Each peristimulus time histogram was constructed from 200 pulses of $0.5 \mathrm{~ms}$ delivered at $1 \mathrm{~Hz}$ at time 0 with an intensity of $300 \mu \mathrm{A}$. Bin width is $2 \mathrm{~ms}$. 8 Histograms showing the effect of the intravenous administration of desipramine in 16 experiments. The SIL values represent the duration of suppression of firing. Data are mean \pm SEM. $\bullet P<0.001$, using a two-tailed paired Student's i-test, comparing prior to, and following. desipramine administration.

hanced the efficacy of the stimulation, while high doses $(100$ and $400 \mu \mathrm{g} / \mathrm{kg})$ reduced it. The $2 \mu \mathrm{g} / \mathrm{kg}$ dose of clonidine increased the SIL value from $35 \pm 5$ to $42 \pm 5 \mathrm{~ms}$, while the $10 \mu \mathrm{g} / \mathrm{kg}$ dose produced a more robust enhancement of the suppression of firing activity with the SIL value increasing from $38 \pm 2$ to $50 \pm 2 \mathrm{~ms}$. The higher doses of clonidine ( 100 and $400 \mu \mathrm{g} / \mathrm{kg}$ ) produced the opposite effect with SIL values being reduced from $44 \pm 2$ to $35 \pm 2 \mathrm{~ms}$ and from $52 \pm 4$ to $35 \pm 4 \mathrm{~ms}$, respectively.

That these effects of clonidine were attributable to the activation of $\alpha_{2}$-adrenoceptors was then ascertained by subsequently injecting the $\alpha_{2}$-adrenoceptor blocking agent yohimbine. Both the incremental and the decremental effects of clonidine on 5.HT neurotransmission were antagonized by yohimbine: Doses of yohimbine

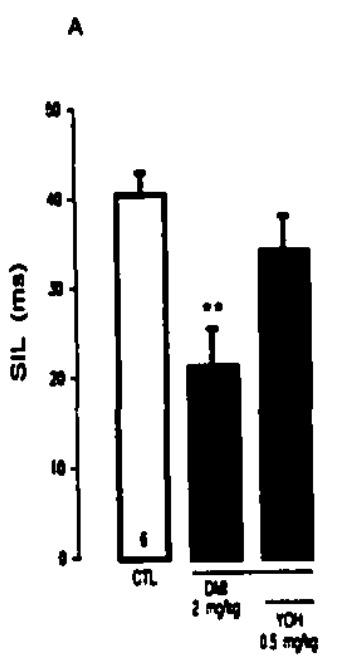

B

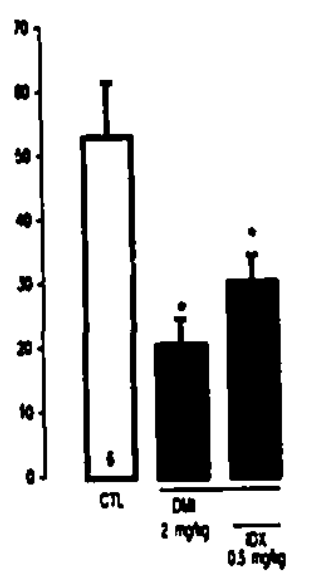

c

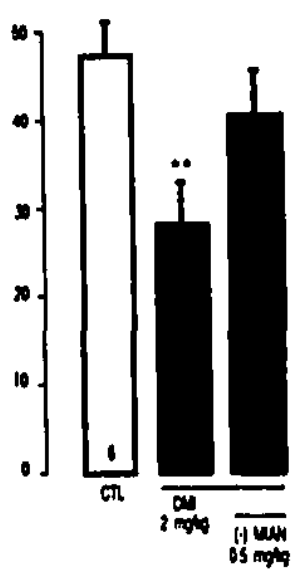

Fis. 2. Histograms illustrating the effect of the intrave. nous administration of the $a_{2}$-adrenoceptor antagonists yohimbine (YOH) A, idazoxan (IDX) B and (-)mians -in ((-)MIAN) $\mathbf{C}$ subsequently to the injection of desipramine on the efficacy of the electrical stimulation of the ascending S.HT pathway. The SIL values represent the duration of suppression of firing. Dau are mean \pm SEM. $\bullet P<0.05$, $\cdots P<0.01$ using a two.tulled paired Student's t-test, comparing prior to, and following, the consecutive administrations of desipramine and of the $a_{2}$-adrenoceptor antagonist. 


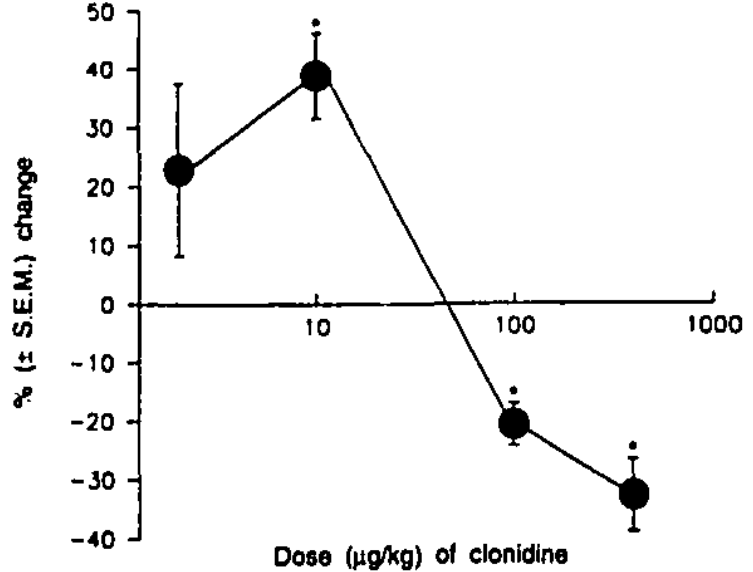

Fig. 3. Esfect of intravenous injections of different doses of clonidine on the efficacy of the electrical stimulation of the ascending 5-HT pathway. The number of rats tested for each dose of clonidine is given within the points. $P<0.001$, two-tailed paired Student's t-test, comparing prior to, and following, clonidine administration

ten times higher than that of clonidine were used: $0.1 \mathrm{mg} / \mathrm{kg}$ of yohimbine antagonized the effect of $10 \mu \mathrm{g} / \mathrm{kg}$ of clonidine and $1 \mathrm{mg} / \mathrm{kg}$ yohimbine antagonized the effect of $100 \mu \mathrm{g} / \mathrm{kg}$ of clonidine (Fig. 4).

\section{Effect of noradrenergic denervation on the modulation of 5-HT neurotransmission by clonidine}

The contention that low doses of clonidine increase 5-HT neurotransmission by attenuating endogenous noradrenaline release, as a result of the activation of $a_{2}$-adrenergic autoreceptors located both on the cell body and terminals of noradrenaline neurons, and that high doses directly activate $\alpha_{2}$-adrenergic heteroreceptors located on 5-HT terminals, was tested in noradrenergic-denervated rats that had received an intracerebroventricular injection
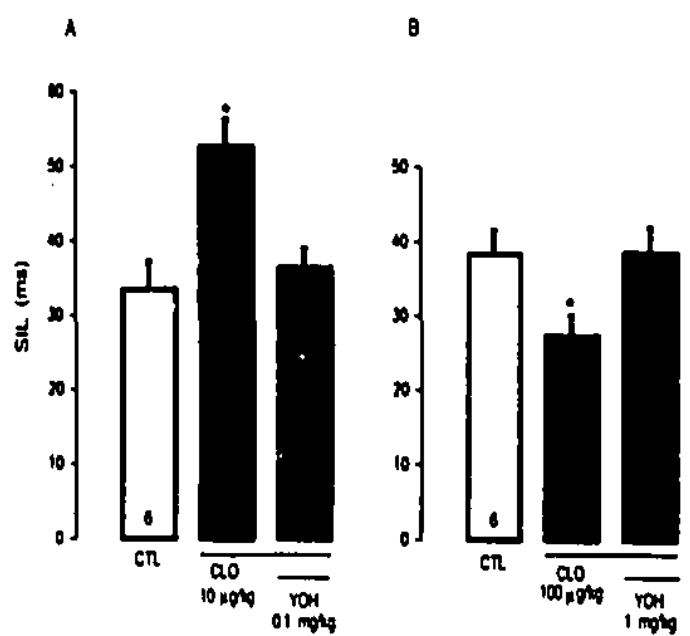

Fir. 4. Effect of the intravenous administration of yohimbine on the ef. ficacy of the stimulation of the ascending S.HT pathway following the intravenous injection of a low (A) and a high (B) dose of clonidine (CLO). Six rats were tested for each dose of clonidine. The SIL values represents the duration of suppression of firing. Data are mean \pm SEM. - $P<0.01$, two-tailed paired Student's $t$-test, comparing prior 10 , and following, clonidine or yohimbine administration of the toxin 6-OHDA two weeks before the experiments. The effectiveness of the lesions was verified by assessing the recovery time ( $\mathrm{RT}_{50}$ ) of the firing rate of $\mathrm{CA}_{3}$ pyramidal neurons following the microiontophosetic application of noradrenaline. In keeping with the notion that intact noradrenaline fibers are required for the noradrenaline reuptake process to allow a prompt recovery of the firing activity of these neurons (de Montigny et al. 1980), the marked increase $\left(488 \%\right.$ ) in the $\mathrm{RT}_{50}$ of 6-OHDA treated rats (Fig. 5A) confirmed an adequate lesioning of the noradrenergic terminals.

The effectiveness of the stimulation of the ascending 5-HT pathway in suppressing the firing activity of the pyramidal neurons was similar in saline and 6-OHDApretreated rats. However, the enhancing effect of the $10 \mu \mathrm{g} / \mathrm{kg}$ dose of clonidine on the efficacy of the stimulation of the ascending 5-HT pathway was abolished by the 6-OHDA pretreatment (Fig. 5B). Whereas, the decrease in the efficacy of the stimulation induced by $100 \mu \mathrm{g} / \mathrm{kg}$ of clonidine was still present in the same rats, indicating that the former, but not the latter, effect is dependent on the presence of noradrenergic terminals. Furthermore, the percentage of inhibition produced by $100 \mu \mathrm{g} / \mathrm{kg}$ of clonidine in the 6-OHDA-pretreated rats $(31 \pm 7 \% ; n=7)$ was greater than that produced in intact rats $(21 \pm 4 \%$; $n=28$ ).

\section{Effect of $\alpha_{2}$-adrenoceptor antagonists on S-HT neurotransmission}

In order to verify whether endogenous noradrenaline attenuates 5-HT neurotransmission under basal conditions, the efficacy of the stimulation of the S-HT pathway was
A

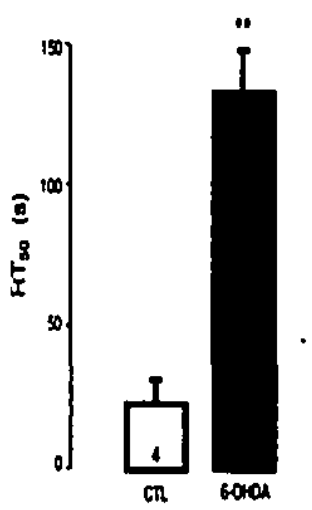

$B$

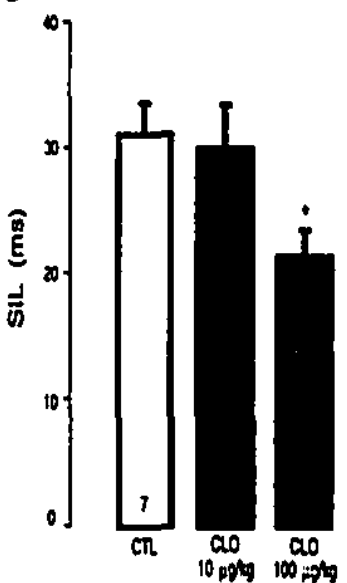

Fig. 5. A Neuronal recovery from the microiontophoretic application of noradrenaline in saline (SAL) and 6-OHDA-pretreater rats. The $\mathrm{RT}_{30}$ value is the time required by the neuron frem the end of the application to recover its firing activity by $50 \%$. The number of neuron tested is given at the bottom of each column. Data are mean \pm SEM. $\cdots P<0.001$, two-tailed Student's t-test comparing control and 6-OHDA-pretreated rats. B Effects of the consecutive administration low and a high dose of clonidine in seven 6-OHDA.piz:ieated rats on the efficacy of the electrical stimulation of the ascending S-HT pathway. The SIL values represents the duration of suppression of firing. Data are mean \pm SEM. - $P<0.01$, two-tailed paired Student's $t$-test, comparing prior to, and following, clonidine administration 
tested prior to, and following, the administration of $0.5 \mathrm{mg} / \mathrm{kg}$ of the $\alpha_{2}$-adrenoceptor antagonists yohimbine, idazoxan and $(-)$ mianserin. As illustrated in Fig. 6A and B, yohimbine and idazoxan had no effect by themselves on 5-HT neurotransmission. However, the selective $\alpha_{2}$-adrenergic heteroreceptor antagonist $(-) \mathrm{mi}$ anserin (Fig. $6 \mathrm{C}$ ) increased by $34 \%$ the duration of suppression of firing produced by the stimulation of the 5-HT pathway.

\section{Discussion}

The purpose of the present study was to determine whether $\alpha_{2}$-adrenoceptors located on 5-HT terminals in the rat hippocampus are tonically activated by endogenous noradrenaline in vivo. The effect of the activation of these $a_{2}$-adrenergic heteroreceptors was therefore studied by comparing the effectiveness of the electrical stimulation of the ascending S-HT pathway in suppressing the firing activity of dorsal hippocampus pyramidal neurons prior to, and following, the intravenous administration of noradrenergic agents. Thus, in this paradigm, the duration of suppression of firing is an indication of the amount of 5-HT released in the synapse. Three different sets of data obtained in the present study support the contention that 5.HT neurotransmission is under tonic inhibitory noradrenergic control: 1) An increase in the synaptic concentration of noradrenaline induced by desipramine decreased the effectiveness of the stimulation of the 5-HT pathway; 2) Low doses of clonidine, that decrease noradrenaline output by activating $\alpha_{2}$-adrenergic autoreceptors on noradrenaline neurons, increased 5-HT neurotransmission; and 3) The blockade of $\alpha_{2}$-adrenergic heteroreceptors by (-)mianserin increased the efficacy of the stimulation of the 5-HT pathway.

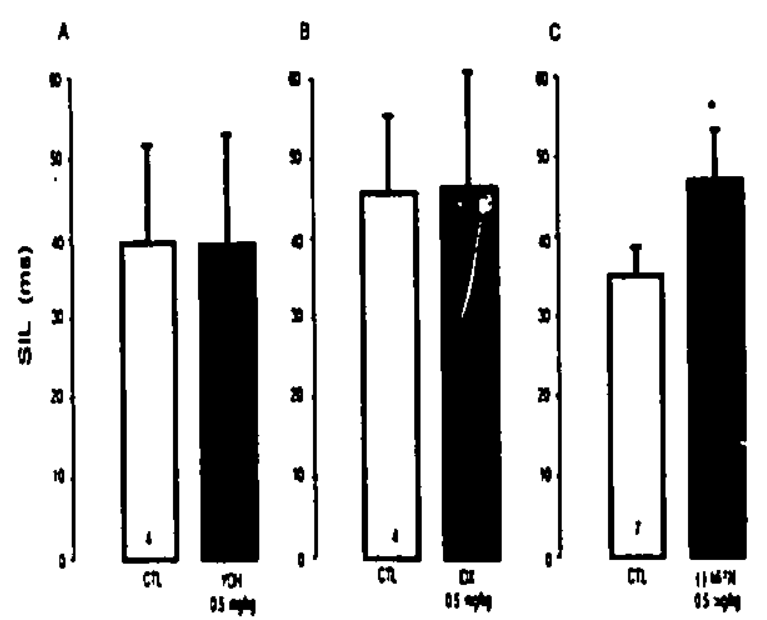

Fig. 6. Histogram illustrating the effect on the efficacy of the electrical stimulation of the ascending5-HT pathway of the intravenous adnutnis:zation of the $a_{2}$-adrenoceptor antagonists yohimbine (A), idazoxan (B) and (..juntan:-:in (C) in the basal condition. The SIL values represents the duration of suppression of firing. Data are mean $\pm S E M$. The number of rats tested is given at the bottom of the open columns. $P<0.05$, iwo-tailed paired Student's $P$-test, comparing prior to, and following, (-)mianserin administration
The results obtained in the present in vivo study following acute blockade of the noradrenaline reuptake pro. cess with tesipramine are at variance with previous in vitro findings. Noradrenaline reuptake blockade reduced the duration of suppression of firing by about $50 \%$ in vivo (Fig. 1), while in vitro it either did not change the evoked release of $\left[{ }^{3} \mathrm{H}\right] 5-\mathrm{HT}$ (Galzin et al. 1984; Benkirane et al. 1985; Limberger et al. 1986) or reduced it by a mere $15 \%$ (Feuerstein et al. 1985). A possible explanation for this apparent discrepancy is that the tonic inhibitory action of endogenous noradrenaline on $\alpha_{2}$-adrenergic heteroreceptors is minimal in in vitro preparations. Endogenous noradrenaline may hardly reach 5-HT terminals because it is washed away by the superfusion flow. Alternatively, the possibility that desipramine exerted its effect in the present experimental paradigm by ail action on receptors other than $\alpha_{2}$-adrenoceptors which affect the function on 5-HT terminals can be excluded since it was reversed with the $\alpha_{2}$-adrenoceptor antagonists yohimbine and (-)mianserin. It is noteworthy that although yohimbine has a moderately high affinity for $5-\mathrm{HT}_{1 \mathrm{~A}}$ binding sites $\left(\mathrm{pK}_{\mathrm{D}}=6.9\right)$, mianserin has a lower affinity for these sites $\left(\mathrm{pK}_{\mathrm{D}}=6.0\right.$; Hoyer, 1991). Furthermore, mianserin does not alter the firing activity of 5-HT neurons nor does it block their somatodendritic

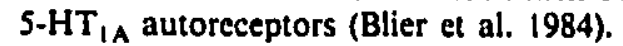

The observation that idazoxan was ineffective in blocking the suppressant effect of desipramine may appear puzzling. First, the possibility that idazoxan does not penetrate the blood brain barrier can readily be excluded because there is ahundant evidence for the capacity of idazoxan, injected intravenously, to antagonizc $\alpha_{2}$ adrenoceptors at doses much Inwer than the one used here on locus coeruleus neuroris both at the level of their cell body and nerve terminals (Washburn and Moises 1989; Curet and de Montigny 1989). Second, there is the possibility that idazoxan could act as a partial agonist at $\alpha_{2}$-adrenoceptors as it was reported to occur with low levels of endogenous noradrenaline (Limberger and Starke 1983). However, this is probably not the case here since the level of noradrenaline was elevated through reuptake blockade by desipramine. Another explanation could be that idazoxan is less potent at $\boldsymbol{\alpha}_{2}$-adrenoceptors on 5-HT fibers than on noradrenergic fibers. This would be consistent with a previous in vitro study showing that idazoxan displays an affinity for autoreceptors on noradrenergic terminals about 250 -fold greater than for adrenoceptors on 5-HT terminals (Preziosi et al. 1989). Furthermore, idazoxan has a high affinity for imidazoline recognition sites. Evidence has been presented suggesting the existence of a non-catecholamine endogenous ligand for imidazoline sites and that noradrenaline would act exclusively at the $\alpha_{2}$-adrenergic phenylethylamine sites (Atlas et al. 1987; Ernsberger et al. 1988; Tibiriça et al. 1991). In keeping with this, our results suggest that $\alpha_{2-}$ adrenoceptors on S.HT terminals would be of the $\alpha_{2}$ adrenergic phenylethylamine type and would be activated by endogenous noradrenaline, but not by a putative endogenous imidazoline ligand.

The biphasic effect of the $\alpha_{2}$-adrenoceptor agonist clonidine on 5-HT neurotransmission is also consistent 
with the notion that $\alpha_{2}$-adrenergic heteroreceptors and $\alpha_{2}$-adrenergic autoreceptors would not be identical, consistent with previous reports (Frankhuyzen and Mulder 1982; Maura et al. 1985) shoving that the potency of clonidine is 10 times higher at the $\alpha_{2}$-adrenergic autoreceptors than at the $\alpha_{2}$-adrenergic heteroreceptors in the rat hippocampus, cerebral cortex and hypothalamus. The enhancing effect of low doses of clonidine on 5-HT neurotransmission is at variant with the in vivo microdialysis results of Tao and Hjorth (1992) and Yoshioka et al. (1992) who found in the rat hippocampus an inhibitory effect of clonidine on 5-HT release at high doses, but no change with low doses of clonidine. That the enhancing effect of a low dose of clonidine in the present study was $\alpha_{2}$-adrenergic in nature is corroborated by its blockade with the $\alpha_{2}$-adrenoceptor antagonist yohimbine. A possible explanation for the discrepancy might be that the electrophysiological paradigm use herein is more suitable than microdialysis to detect the effect of local changes in noradrenaline availability on S-HT neurotransmission. In support of that contention, the observation that the enhancing effect of the low dose of clonidine $(i j \mu \mathrm{g} / \mathrm{kg}$ ) was abolished in 6-OHDA-pretreated rats (Fig. 5B) indicates that this effect of clonidine is attributable to the activation of $\alpha_{2}$-adrenergic autoreceptors, resulting in a decreased tonic activation of $\alpha_{2}$-adrenergic heteroreceptors by endogenous noradrenaline. Contrary to the enhancing effect of the low dose of clonidine $(10 \mu \mathrm{g} / \mathrm{kg})$, the inhibitory effect of the high dose clonidine $(100 \mu \mathrm{g} / \mathrm{kg})$ on $5-\mathrm{HT}$ neurotransmission was not abolished by the 6-OHDA treatment suggesting that the high dose of clonidine activates directly the $\alpha_{2}$-adrenergic heteroreceptors to decrease 5-HT release.

The inhibitory effect of the high dose $(100 \mu \mathrm{g} / \mathrm{kg})$ of clonidine was slightly increased in 6-OHDA-pretreated rats, consistent with previous reports of an increased responsiveness to agonist stimulation of $a_{2}$-adrenergic heteroreceptors following noradrenaline denervation with the toxin DSP4 (Benkirane et al. 1985; Ellison and Campbell 1986). However, these results are at variant with studies showing that the responsiveness of $\alpha_{2}$-adrenergic heteroreceptors to clonidine (Yoshioka et al. 1992) or to exogenous noradrenaline (Schlicker et al. 1982) is not altered following denervation with 6-OHDA. There is the possibility that DSP4 produces a greater depletion of noradrenaline than 6-OHDA. If this holds true, the increased effect observed after DSP4 might be explained by a decreased competition of the exogenous agonist with endogenous noradrenaline instead of a change in the sensitivity of the receptor per se (Gothert and Schlicker 1991). Furthermore, it may appear surprising that the effectiveness of the stimulation of the ascending 5-HT pathway in the 6-OHDA-pretreated rats was not greater in saline-pretreated rats considering that the inhibitory tone of endogenous noradrenaline on 5-HT neurotransmission should have been removed. Consistently, Schlicker et al. (1982) and Yoshioka et al. (1992) found no change in the evoked release of S.HT in their control condition following a noradrenaline depletion with 6-OHDA. Three explanations can be envisaged to account for this apparent discrepancy in these results and/or the present study: 1) The toxin 6-OHDA not being entirely specific for the catecholaminergic fibers could have lesioned some of the 5-HT fibers resulting in a reduction in 5-HT neurotransmission. However, this is not likely since the 5-HT carrier was blocked with fluoxetine $\mathrm{I} \mathrm{h}$ before the administration of the toxin. Such a procedure is known to effectively protect the rat brain from 5-HT.depletion (Gravel and de Montigny 1987); 2) A change in 5-HT neurotransmission could have remained undetected due to the small number of rats tested. However, this is not probable since an expected change of about $40 \%$ (similar to that observed following the acute administration of $10 \mu \mathrm{g} / \mathrm{kg}$ of clonidine) should have been readily detected; 3 ) Compensatory mechanisms may have entered into play. For instance, 5-HT autoreceptors could have maintained the efficacy of 5-HT neurotransmission in the normal range despite the changes in the tonic inhibitory effect of endogenous noradrenaline on $\alpha_{2}$-adrenergic heteroreceptors.

Another important issue is the possibility that a tonic inhibitory effect of endogenous noradrenaline on $\alpha_{2}$-adrenergic heteroreceptors could be blocked by $\alpha_{2}$-adrenoceptor antagonists. We did not find any change in the efficacy of the stimulation of the S-HT pathway in the rat hippocampus following the administration of idazoxan in naive rats, in keeping with the findings of Tao and Hjorth in the same species and tissue (1992). Even though yohimbine was effective in blocking the inhibitory effect of increased endogenous noradrenaline concentration induced by desipramine, it did not antagonize the putative inhibitory action of endogenous noradrenaline on 5-HT neurotransmission in the basal condition. One plausible explanation for these results might be that while yohimbine is expected to increase 5 -HT release by blocking $\alpha_{2}$-adrenergic heteroreceptors, it also blocks $\alpha_{2}$-adrenergic autoreceptors on noradrenergic neurons increasing endogenous noradrenaline release and consequently decreasing 5-HT release. In the basal condition the two phenomena would cancel each other, thus resulting in an unchanged 5-HT neurotransmission. The enhanced 5-HT neurotransmission obtained with the specific $\alpha_{2}$-adrenergic heteroreceptor antagonist (-)mianserin supports this possibility. The (-)stereoisomer of mianserin presumably blocked the $\alpha_{2}$-adrenergic heteroreceptors without affecting the $a_{2}$-adrenergic autoreceptors (Maura et al. 1985; Raiteri et al. 1983), thus revealing the presence of a tonic inhibitory action of endogenous noradrenaline on 5-HT neurotransmission.

The results of the present study indicate that the noradrenaline system interacts in vivo with the 5-HT system via $\alpha_{2}$-adrenoceptors located on 5-HT terminals. This interaction might be clinically important in the mechanism of action of antidepressant drugs which augment the availability of noradrenaline in the synapse. Preliminary resuits suggest that such antidepressant alter the sensitivity of these $\alpha_{2}$-adrenergic heteroreceptors (Mongeau et al. 1992).

Acknowledgements. This work was supported in past by the Medical Research Council of Canada (MRC) grants MT-6144 and 11014 and the Fonds de la recherche en sante du Quebec (FRSQ). R.M. is in receipt of a Studentship from FRSQ and P. B. of a Scholarship from MRC. 


\section{References}

Atlas D, Diamant S, Fales HR, Pannel L (1987) The brain's own clonidine: purification and characterization of endogenous clonidine displacing substance from brain. J Cardiol Pharmacol 10:S122-S127

Benkirane S, Arbilla S, Langer S (1985) Supersensitivity of $a_{2}$-adrenoceptors modulating [ $\left.{ }^{3} \mathrm{H}\right] 5$-HT release after noradrenergic denervation with DSP4. Eur J Pharmacol 119:131-133

Blier P, de Montigny C. Tardif D (1984) Effects of the two antidepressant drugs mianserin and indajpine on the serotonergic system: Single-cell studies in the rat. Psychopharmacology 84:242-249

Chaput Y, de Montigny C, Blier P (1986) Effects of a selective S-HT reuptake blocker, citalopram, on the sensitivity of S-HT autoreceptors: Electrophysiological studies in the rat brain. NaunynSchmiedeberg's Arch Pharmacol 333:342-348

Curet O. de Montigny C (1989) Electrophysiological characterization of adrenoceptors in the rat dorsal hippocampus. III. Evidence for the physiological role of terminal $\alpha_{2}$-adrenergic autoreceptors. Brain Res 499:18-26

de Montigny C, Wang RY, Reader TA, Aghajanian GK (1980) Monoaminergic denervation of the rat hippocampus: Microiontophoretic studies on pre- and postsynaptic supersensitivity to noradrenaline and serotonin. Brain Res 200:363-376

Ellison DW, Campbell IC (1986) Studies on the role of $a_{2}$-adrenoceptors in the control of synaptosomal $\left.{ }^{3} \mathrm{H}\right] \mathrm{S}$-hydroxytryptamine release: Effects of antidepressant drugs. J Neurochem 46:218-223

Ernsberger P, Meeley MP, Reis DJ (1988) An endogenous substance with clonidine-like properties: Selective binding to imidazole sites in the ventrolateral medulla. Brain Res 441:309-318

Feuerstein TJ, Hertting G, Jackisch R (1985) Endogenous noradrenaline as modulator of hippocampal serotonin (S-HT) release. NaunynSchmiedeberg's Arch Pharmacol 329:216-221

Frankhuijzen A, Mulder AH (1982) Pharmacological characterization of presynaptic $\alpha$-adrenoceptors modulating $\left({ }^{3} \mathrm{H}\right)$ noradrenaline and $\left[{ }^{3} \mathrm{H}\right] \mathrm{s}$-hydroxytryptamine release from slices of the hippocampus of the rat. Eur J Pharmacol 81:97:106

Frankhuijzen AL, Wardeh G, Hogenboom F, Mulder AH (1988) Alpha-2 adrenoceptor mediated inhibition of the release of radiolabelled S-hydroxytryptamine and noradrenaline from slices of the dorsal region of the rat brain. Naunyn-Schmiedeberg's Arch Pharmacol 337:255-260

Galzin AM, Motet C, Langer SZ (1984) Evidence that exogenous but not endogenous noradrenaline activates the presynaptic alpha-2 adrenoceptors on serotonergic nerve endings in the at hypothalamus. J Pharmacol Exp Ther 228:725-732

Gobbi M, Frittoli E, Mennini T (1990) The modulation of $\left[{ }^{3} \mathrm{H}\right]$ noradrenaline and $\left[{ }^{3} \mathrm{H}\right]$ serotonin release from rat brain synaptosomes is not mediated by the $\alpha_{2 B^{-a d r e n o c e p t o r}}$ subtype. NaunynSchmiedeberg's Arch Pharmacol 342:382-386

Goldberg MR, Robertson D (1983) Yohimbine: A pharmacological probe for study of the alpha $a_{2}$-adrenoceptors. Pharm Rev 35:143-170

Gothert M. Huth H (1980) Alpha-adrenoceptor-mediated modulation of S-hydroxytryptamine release from rat brain cortex slices. Naunyn-Schmiedeberg's Arch Pharmacol 313:21-26

Gothert M. Huth H. Schlicker E (1981) Characterization of the receptor subtype involved in alpha-adrenoceptor-mediated modulation of se. rotonin release from rat brain cortex slices. Naunyn-Schmiedeberg's Arch Pharmacol 317:199-203

Gothert M. Schlicker E (1991) Regulation of serotonin release in the central nervous system by presynaptic heteroreceptors. In: Feigenbaum J. Hanani M (eds) Presynaptic regulation of neurotransmitter release: A handbook. Freund, Tel Aviv, pp 845-876

Gravel P, de Montigny C (1987) Noradrenergic denervation prevents sensitization of rat forebrain neurons to serotonin by tricyclic anti. depressant treatment. Synapse 1:233-239

Hamilton CA, Reid JL, Yakubu MA (1988) $\int^{3} \mathrm{H}$ ]Yohimbine and ['H]idazoxan bind to different sites on rabbit forebrain and kidney membranes. Eur J Pharmacol 146:345 - 348

Hoyer D (1991) The 5.HT receptor family: Ligands, distribution and receptor-effector coupling. In: Rodgers RJ, Cooper SJ (eds) S-HTIA agonists, 5.HT3 antagonists and benzodiazepines: Their comparative behavion! pharmacology. Wiley, Chichester, pp $31-57$

Kandel ER, Spencer WA (1961) Electrophysiology of hippocampal neurons. II. Afterpotentials and repetitive firing. Psychopharmacology 83:20-27

Limberger N. Bonanno G, Spath L, Starke K (1986) Autoreceptors and $a_{2}$-adrenoceptors at the serotonergic axons of rabbit brain cortex. Naunyn-Schmiedeberg's Arch Pharmacol 332:324-331

Limberger N. Fischer MRG, Wichmann T. Starke $K(1989)$ Phencolamine blocks presynaptic serotonin autoreceptors in rabbit and rat brain cortex. Naunyn-Schmiedeberg's Arch Pharmacol 340:52-61

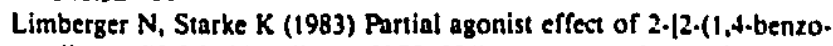
dioxanyl)]-2-imidazoline (RX 781094$)$ at presynaptic $a_{2}$-adrenoceptors in rabbit ear artery. Naunyn-Schmiedeberg's Arch Pharmacol 324:75-78

Maura G. Gemignani A, Raiteri $M$ (1985) $\alpha_{2}$-Adrenoceptors in rat hy. pothalamus and cerebral cortex: Functional evidence for pharmacologically distinct subpopulations. Eur J Pharmacol 116:335 - 339

Maura G, Bonanno G, Raiteri M (1992) Presynaptic $a_{2}$-adrenoceptors mediating inhibition of noradrenaline and s-hydroxyıryptamine re. lease in rat cerebral cortex: Further characterization as different $a_{2}$-adrenoceptor subtypes. Naunyn-Schmiedeberg's Arch Pharmacol 345:410-416

Michel MC, Regan JW, Gerhardt MA, Neubig RR, Insel PA, Motulsky HJ (1990) Noradrenergic [ ${ }^{3} \mathrm{H}$ lidazoxan binding sites are physically distinct from alpha $a_{2}$-adrenergic receptors. Mol Pharmacol 37:65-68

Mongeau R, Blier P, de Montigny C (1992) Electrophysiological evidence for the desensitization of $a_{2}$-adrenergic heteroreceptors on serotonin terminals in the rat hippocampus following chronic antidepressant treatments. Soc Neurosci Abstr 18:95.1

Preziosi P, Martire M, Navarra P, Pistritto G, Vacca M (1989) Prolactinlowering ability of $( \pm)$-idazoxan may be linked to a central noradrenergic-serotonergic interplay. J Pharmacol Exp Ther 249:256-263

Raiteri M, Maura G, Folghera S, Cavazzani P, Andrioli GC, Schlicker E, Schalnus R, Gothert M (1990) Modulation of 5.hydroxytryptamine release by presynaptic inhibitory alpha-2-adrenoceptors in the human cerebral cortex. Naunyn-Schmiedeberg's Arch Pharmacol 342:508-512

Raiteri M. Maura G, Versace P (1983) Functional eviden for two stereochemically different alpha-2 adrenoceptors regulatin. entral noradrenaline and serotonin release. J Phas:macol Ex, Ther 224:679-684

Ross SB, Renyi AL (1975) Tricyclic antidepressant agents. II. Efrects of oral administration on the uptake of ${ }^{3} \mathrm{H}$-noradrenatine and ${ }^{14} \mathrm{C}$-5.hydroxytryptamine in stices of the midbrain-hypothalamus region of the rat. Acta Pharmacol Toxicol 36:395-408

Sclilicker E, Gothert M, Clausing R (1982) Acute or chronic changes of noradrenergic neurotransmission do not affect the a-adrenoceptor-mediated intibition of ${ }^{3} \mathrm{H}$-serotonin release in the cerebral cortex. Naunyn-Schmiedeberg's Arch Pharmacol 320:38-44

Schlicker E, Gothert M, Kostermann F, Clausing R (1983) Effects of $a$ adrenoceptor antagonists on the release of serotonin and noradrena. line from rat brain cortex slices. Naunyn-Schmiedeberg's Arch Pharmacol 323:106-113

Tho R, Hjorth $S$ (1992) $\alpha_{2}$-Adrenoceptor modulation of rat ventral hippocampal S-hydroxytryptamine release in vivo. Naunyn-Schmiede. berg's Arch Pharmacol 345:137-143

Tibiriça E, Feldman J, Mermet C, Gonon F, Bousquet P (1991) An imidazoline-specific mechanism for the hypotensive effect of clonidine: A study with yohimbine and idazoxan. J Pharm Exp Ther 256:606-613

Washburn M. Moises HC (1989) Electrophysiological correlates of presynaptic $a_{2}$-receptor-mediated inhibition of norepinephrine release at locus-coeruleus synapses in dentate gyrus. I Neurosci 9:2131-2140

Yoshioka M, Matsumoto M, Togashi H, Smith CB, Sajto H (1992) Er. feet of clonidine on the release of serotonin from the rat hippocam. pus as measured by microdialysis. Neurosci Lett 139:57-60 


\section{Second article}

We have then undertaken to determine whether long-term antidepressant treatments would affect the responsiveness of the $\alpha_{2}$-adrenoceptors modulating the release of 5-HT using the electrophysiological paradigm of the previous study. Our working hypothesis was that there might be a decreased inhibitory effect of endogenous NA at $\alpha 2$-adrenergic heteroreceptors after antidepressant treatments, which would result from either a desensitization of $\alpha_{2}$-adrenergic heteroreceptors or from a reduced input of endogenous NA acting at these heteroreceptors, or both. The sensitivity of the $\alpha_{2}-$ adrenergic heteroreceptors was tested with a high dose of clonidine $(400 \mu \mathrm{g} / \mathrm{kg}$ i.v.) and the degree of tonic inhibition by endogenous NA was assessed by measuring the enhancement in 5-HT neurotransmission produced by activating the $\alpha_{2}$-adrenergic autoreceptors with a small dose of clonidine $(10 \mu \mathrm{g} / \mathrm{kg}$, i.v.). The sensitivity of postsynaptic 5 - $\mathrm{HT}_{1 \mathrm{~A}}$ receptors and $\alpha_{2}$-adrenoceptors was aiso tested by assessing the suppressant effect on $\mathrm{CA}_{3}$ pyramidal neurons of microiontophoretically-applied 5-HT and NA. Members of three major classes of antidepressant drugs were studied, that is, selective inhibitors of 5-HT or NA reuptake and a type A MAOI .

This article entitled "Electrophysiological evidence for desensitization of a2adrenoceptors on serotonin terminals following long-term treatment with drugs increasing norepinephrine synaptic concentration" by myself, Claude de Montigny and Pierre Blier was published in Neuropsychopharmacology (1994, vol. 10, pp. 41-51). 


\section{Electrophysiologic Evidence for Desensitization of $\alpha_{2}$-Adrenoceptors on Serotonin Terminals Following Long-Term Treatment with Drugs Increasing Norepinephrine Synaptic Concentration}

Raymond Mongeau, M.Sc., Claude de Montigny, M.D., Ph.D., and Pierre Blier, M.D., Ph.D.

Previous results from our laboratory have indicated that small intravenous doses of the $\alpha_{2}$-adrenergic agonist clonidine increase serotonin (5-HT) neurotransmission by attenuating the release of endogenous norepinephrine $(N E)$, as a result of the activation of $\alpha_{2}$-adrenergic autoreceptor on NE neurons, and that high doses of clonidine decrease 5-HT neurotransmission by directly activating $\alpha_{2}$-adrenergic heteroreceptors on 5-HT terminals. The aim of the present study was to assess whether antidepressant treatments that increase the synaptic concentration of NE or 5-HT alter the ability of clonidine to modulate 5-HT neurotransmission through these two $\alpha_{2}$-adrenoceptors. Rats were treated for 3 weeks with $0.75 \mathrm{mg} / \mathrm{kg}$ per day of befloxatone (a reversible inhibitor of monoamine oxidase $A), 10 \mathrm{mg} / \mathrm{kg}$ per day of nisoxetine (a selective NE reuptake inhibitor), $10 \mathrm{mg} / \mathrm{kg}$ per day of paroxetine (a selective 5-HT reuptake inhibitor) or saline using subcutaneous osmotic minipumps (removed 48 hours before the experiment). No significant change in the effect of the small dose of clonidine (10 $\mu \mathrm{g} / \mathrm{kg}, \mathrm{IV}$ ) was found following the befloxatone, the nisoxetine, or the paroxetine treatments. The reduction of 5-HT neurotransmission by the high dose of clonidine $(400 \mu \mathrm{g} / \mathrm{kg}, I V)$ was no longer present in rats treated with nisoxetine or befloxatone, but was unaltered in those treated with paroxetine. Furthermore, in rats pretreated with the NE neurotoxin 6-hydroxydopamine, a long-lerm treatment with befloxatone failed to alter the reducing effect of the high dose of clonidine but abolished the reducing effect of the low dose of clonidine. These results suggest that antidepressant drugs that increase $N E$ synaptic concentration induce a desensitization of $\alpha_{2}$-heteroreceptor on 5-HT terminals.

[Neuropsychopharmacology 10:41-51, 1994]
KEY wORDS: $\alpha_{2}$-Adrenergic autoreceptors; $\alpha_{2}$-Adrenergic heteroreceptors; Antidepressant drugs; Norepinephrine reuptake inhibitors; Serotonin reuptake inhibitors; Monoamine oxidase inhibitors

From the Department of Psychiatry, Neurobiological Psychiatry Unit, McGill University, Montréal, Québec, Canada

Address all correspondence to: Raymond Mongeau, M.Sc. Department of Psychiatry, Neurobiological Psychjatry Unit, McGill University, 1033 Pine Avenue West, Montréal, Québec, Canada H3A 1 A1.

'eceived June 29, 1993; revised September 17, 1993; accepted September 26, 1993.
Several studies have documented the possibility that presynaptic $\alpha_{2}$-adnenergic autoreseptors become desensitized following long-term antidepressant treatments that increase the synaptic co.ıcentration of norepinephrine (NE) (Cren's and Smith 1978; Svensson and Usdin 1978; McMillen et al. 1980; Spyraky and Fibiger 1980; Cohen et al. 1982; Finberg and Tal 1985; Lacr jix et al. 1991). Furthermore, the density of high-affinity state $\alpha_{2}$-adrenoceptors has been shown to be increased on platelets of depressed patients, a condition normalized by long-term treatment with antidepressant drugs (Garcia-Sevilla et al. 1986, 1987; Doyle et al. 1985; 
Takeda et al. 1989; Piletz et al. 1991). However, not all antidepressant treatments share the property of decreasing these $\alpha_{2}$-adrenoceptors (Willner 1985). in contrast, several classes of antidepressant treatments have been shown to alter serotonin (5-HT) neurotransmission (for review, see Blier et al., 1990).

The capacity of $\alpha_{2}$-adrenergic ligands to modulate the evoked release of $\left[{ }^{3} \mathrm{H}\right] 5-\mathrm{HT}$ in vitro has long been known to be exerted by $a_{2}$-adrenoceptors located on 5-HT terminals (Starke and Montel 1973; Göthert and Huth 1980; Frankhuyzen and Mulder 1980,1982; Göthert et al. 1981; Maura et al. 1982). Using an in vivo electrophysiologic paradigm, it was recently shown that these $\alpha_{2}$-adrenergic hete:oreceptors in the rat hippocampus are tonically activated by endogenous NE (Mongeau et al. 1993), as is the case in the human brain (Galzin et al. 1492; Feuerstein et al. 1993). Small doses $\left(2 \mu \mathrm{g} / \mathrm{kg}\right.$ and $10 \mu \mathrm{g} / \mathrm{kg}$ IV) of the $\alpha_{2}$-adrenergic agonist clonidine enhance 5-HT neurotransmission, an effect that is abolished by a 6-hydroxydopamine (6-OHDA) pretreatment and blocked by the $\alpha_{2}$-adrenergic antagonist yohimbine $(0.1 \mathrm{mg} / \mathrm{kg}$ IV). These results indicate that a low dose of clonidine preferentially activates $\alpha_{2}$-adrenergic autoreceptors on NE neurons, reducing the tonic inhibitory action of endogenous NE on $\alpha_{2}$ adrenergic heteroreceptors on 5-HT terminals and, consequently, increasing 5-HT release. However, at higher doses ( $100 \mu \mathrm{g} / \mathrm{kg}$ and $400 \mu \mathrm{g} / \mathrm{kg}$ IV), clonidine decreases 5-HT neurotransmission through a direct activation of $\alpha_{2}$-adrenergic heteroreceptors, as this effect is not affected by NE denervation and is blocked by yohimbine (1 mg/kg IV; Mongeau at al. 1993).

In the present study, this in vivo modulation of $5-\mathrm{HT}$ neurotransmission by clonidine via $\alpha_{2}$-adrenergic autoand heteroreceptors was used to investigate the effect of long-term treatments that increase the synaptic concentration of NE or 5-HT. The changes in the synaptic concentration of the neurotransmitters were induced by either selective reuptake blockade or monoamine oxidase A (MAO-A) inhibition. This electrophysiologic paradigm was considered most appropriate to investigate the modulation of 5-HT neurotransmission by the NE system because, as described before, it allows the in vivo assessment of the responsiveness of the $\alpha_{2}$ adrenergic heteroreceptors on 5-HT terminals and of the level of tonic inhibitory action of NE acting on them.

\section{METHODS}

\section{Animals and Treatments}

Male Sprague-Dawley rats, maintained on a 12-hour/12hour light-dark cycle with free access to food and water, were treated over a course of 3 weeks with 0.75 $\mathrm{mg} / \mathrm{kg}$ per day of the reversible MAO-A inhibitor beflox- atone (Curet et al. 1992a), $10 \mathrm{mg} / \mathrm{kg}$ per day of the selective 5-HT reuptake inhibitor paroxetine (Thomas et al. 1987), $10 \mathrm{mg} / \mathrm{kg}$ per day of the selective NE reuptake inhibitor nisoxetine (Fuller et al. 1979), or saline delivered by osmotic minipumps (ALZA, Palo Alto, CA) inserted subcutaneously. The minipumps were removed 48 hours before the experiments began to allow elimination of the drugs. Lesions of NE neurons were performed under chloral hydrate anesthesia $(400 \mathrm{mg} / \mathrm{kg}$ IP) by injecting 6-OHDA intracerebroventricularly ( 120 $\mu \mathrm{g}$ free base in $20 \mu \mathrm{l}$ of $0.9 \% \mathrm{NaCl}$ and $0.1 \%$ ascorbic acid) 1 hour after an injection of tluoxetine $(10 \mathrm{mg} / \mathrm{kg}$, IP) administered to protect the 5-HT system from the neurotoxic action of 6-OHDA. One week after this procedure, the rats were treated with $0.75 \mathrm{mg} / \mathrm{kg}$ per day of befloxatone for 3 weeks. At the time of the experiments, the rats (weighing between 325 and $375 \mathrm{~g}$ ) were anesthetized with an injection of $400 \mathrm{mg} / \mathrm{kg}$ (IP) of chloral hydrate.

\section{Recording from Dorsal Hippocampus $\mathrm{CA}_{3}$ Pyramidal Neurons}

Extracellular unitary recording and microiontophoresis onto pyramidal neurons in the $\mathrm{CA}_{3}$ region of the dorsal hippocampus were conducted with five-barrelled micropipettes, pulled conventionally with the tip broken to a diameter of $9 \mu \mathrm{m}$ to $11 \mu \mathrm{m}$. The central bartel, used for recording, was filled with a $2 \mathrm{~mol} / \mathrm{L} \mathrm{NaCl}$ solution. The side barrels contained the following solutions: $5-\mathrm{HT}$ creatinine sulfate $(0.5 \mathrm{mmol} / \mathrm{L}$ in $200 \mathrm{mmol} / \mathrm{L}$ $\mathrm{NaCl}, \mathrm{pH} 4), \mathrm{NE}$ bitartrate $(20 \mathrm{mmol} / \mathrm{L}$ in $200 \mathrm{mmol} / \mathrm{L}$ $\mathrm{NaCl}, \mathrm{pH} 4)$, acetylcholine chloride $(20 \mathrm{mmol} / \mathrm{L}$ in 200 $\mathrm{mmol} / \mathrm{L} \mathrm{NaCl}, \mathrm{pH} 4$ ), and a $2-\mathrm{mol} / \mathrm{L} \mathrm{NaCl}$ solution used for automatic current balancing. The 5-HT and NE solutions were retained with a $-7 n \mathrm{~A}$ current between ejections. Pyramidal neurons were identified by their large amplitude $(0.5 \mathrm{mV}$ to $1.2 \mathrm{mV})$ and long-duration (0.8 msec to $1.2 \mathrm{msec}$ ) simple spikes alternating with complex spike discharges (Kandel and Spencer 1961). These characteristics readily allow the differentiation of pyramidal neurons from interneurons. A small current or leak of acetylcholine ( $-1 \mathrm{nA}$ to $5 \mathrm{nA}$ ) was used to activate silent or slowly discharging pyramidal neurons to a physiologic fring rate $(8 \mathrm{~Hz}$ to $12 \mathrm{~Hz})$, because most of these cells do not discharge spontaneously in chloral hydrate-anesthetized rats. The responsiveness to microiontophoretic application of 5-HT and NE was evaluated from the number of spikes suppressed/nA calculated by an on-line computer with a 100 -millisecond discrimination.

To evaluate the effectiveness of the 6-OHDA lesion, the recovery of the fring activity of the pyramidal neurons following the microiontophoretic application of $\mathrm{NE}$ was assessed by determining the recovery time 50 
$\left(R T_{50}\right)$. This parameter is defined by the time (in seconds) required for the firing rate to recover by $50 \%$ from the termination of the microiontophoretic application (de Montigny et al. 1980).

\section{Stimulation of the 5-HT Pathway}

To activate the 5-HiT projections to the dorsal hippocampus, a bipolar electrode (NE-100; David Kopf, Tujunga, CA) was implanted on the nudline with a $10^{\circ}$ backward angle in the ventromedial tegmentum, $1 \mathrm{~mm}$ anterior to lambda, and $8.3 \mathrm{~mm}$ below the cortical surface. Two hundreds square pulses of 0.5 milliseconds were delivered by a stimulator (Model S8800; Grass, Quincy, MA) at a frequency of $1 \mathrm{~Hz}$ and at an intensity of $300 \mu \mathrm{A}$. The stimulation pulses and the firing activity of the neuron recorded were fed to a computer equipped with a Tecmar interface. Peristimulus time histograms were generated to determine the duration of suppression of firing, measured to absolute silence value (SIL, in milliseconds; Chaput et al. 1986). This parameter is obtained by dividing the total number of events suppressed with the stimulation by the mean frequency of firing of the neuron recorded. It thus represents an estimate of the duration of the suppression of firing corrected for the rean prestimulation firing frequency. Several lines of evidence indicate that the effect of the electrical stimulation of the ascending 5-HT pathway is due to the release of 5-HT into the synaptic cleft. First, it is virtually abolished by a pretreatment with the 5-HT neurotoxin 5,7-dihydroxytryptamine (Blier and de Montigny 1983, 1985). Second, it is blocked by the acute intravenous injection of the 5- $\mathrm{HT}_{1 \mathrm{~A}}$ receptor antagonist BMY 7378 (Yocca et al. 1987; Chaput and de Montigny 1988). Third, it is enhanced by terminal 5-HT autoreceptor blockade (Chaput et al. 1986). Fourth, it is reduced by terminal 5-HT autoreceptor activation (Chaput and de Montigny 1988).

In most neurons, following the suppression of firing induced by the electrical stimulation there is a transient increase of the probability of firing, the exact nature of which is presently unknown. However, this transient increase was shown to be unaffected by 5 -HT reuptake inhibitors or MAO inhibitors (Blier et al. 1988). The relative activation was calculated by dividing the total number of supplementary events by the mean prestimulation firing frequency.

The effect of stimulating the ascending 5- $\mathrm{HT}$ fibers was determined while recording from the same neuron before and after the successive intravenous injections of $10 \mu \mathrm{g} / \mathrm{kg}$ and $400 \mu \mathrm{g} / \mathrm{kg}$ of clonidine. The time elapsed between the control stimulation periods and those following the administration of clonidine was approximately 15 minutes.

\section{Drugs}

The following drugs were used: befloxatone (Delalande, Rueil-Malmaison, France); paroxetine (SmithKline Beecham, Harlow, England); nisoxetine and fluoxetine (Eli Lilly, Indianapolis, IN); clonidine $\mathrm{HCl}$, 7- $\mathrm{HT}$ creatinine sulfate, NE bitartrate, 6-OHDA $\mathrm{HCl}$, and acetylcholine chloride (Sigma Chemical, St. Louis, $\mathrm{MO}$ ); and chloral hydrate (American Chemicals, Montréal, Québec, Canada).

\section{Statistical Analyses}

All results are expressed as means plus/minus standard error of the mean. The statistical significance of the difference between the effects of the stimulation of the 5-HT pathway before and after the administration of a drug in the same rat was assessed using the paired Student's $t$-test. Possible differences in the magnitude of the effects of clonidine between saline- and drugtreated rats were tested for statistical significance by covariance analysis. Differences in the efficacy of the stimulation of the 5-HT pathway and neuronal responsiveness to microiontophoretic application of NE and 5-HT between saline- and d $:$ treated rats were assessed for statistical significance using the unpaired Student's $t$-test.

\section{RESULTS}

\section{Effect of Long-Term MAO Inhibition on the} Modulation of 5-HT Neurotransmission by Clonidine

The suppressant effect of the electrical stimulation of the ascending 5-HT pathway on the firing rate of $\mathrm{CA}_{3}$ pyramidal neurons of the dorsal hippocampus was assessed following long-term drug treatments. The peristimulus time histograms in Figure $1 \mathrm{~A}$ show that $10 \mu \mathrm{g} /$ $\mathrm{kg}$ IV of clonidine enhanced, while $400 \mu \mathrm{g} / \mathrm{kg}$ iV reduced, the duration of suppression of fring in the same neuron of a saline-treated rat. Following the administration of $10 \mu \mathrm{g} / \mathrm{kg}$ and $400 \mu \mathrm{g} / \mathrm{kg}$ IV of clonidine, the mean duration of suppression of firing of the salinetreated group (Fig. 1B) was increased by $37 \% \pm 8 \%$ and decreased by $25 \% \pm 7 \%$, respectively. These incremental and decremental effects of clonidine were statistically significant at $p<.01$ and $p<.001$, respectively, using the paired Student's $t$-test. In contrast, the facilitatory effect on the firing rate of pyramidal neurons induced by the electrical stimulation was not significantly changed by either dose of clonidine (Table 1).

To induce a sustained increase in the synaptic concentration of 5-HT and NE, MAO-A was blocked over a 3-week period with $0.75 \mathrm{mg} / \mathrm{kg}$ per day of befloxatone. The minipumps were removed 48 hours before 


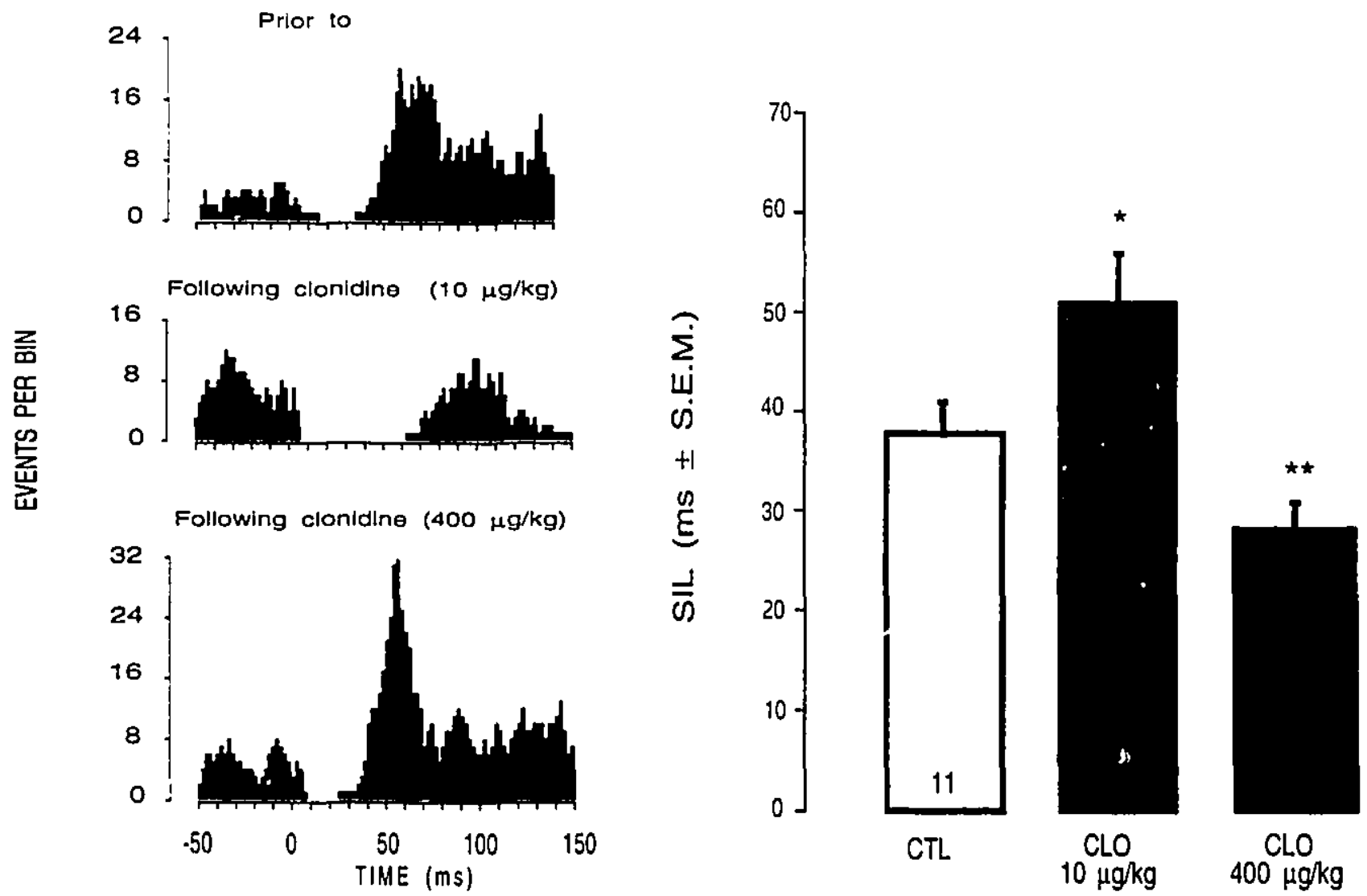

Figure 1. Effect of systemic injections of clonidine on the efficacy of the electrical stimulation of the ascending 5-HT pathway in suppressing the fring activity of $\mathrm{CA}_{3}$ pyramidal neurons of hippocampus in the saline-treated group. A: Representative peristimulus time histogr $\mathrm{ms}$ illustrating the effects of clonidine $(10 \mu \mathrm{g} / \mathrm{kg}$ and $400 \mu \mathrm{g} / \mathrm{kg}$ IV) in a single experiment. Each peristimulus time histogram was constructed from 200 pulses of $0.5 \mathrm{msec}$ delivered at $1 \mathrm{~Hz}$ at time 0 with an intensity of $300 \mu \mathrm{A}$. Bin width is $2 \mathrm{msec}$. B: Histograms showing the effects of the high and the low dose of clonidine in saline-treated rats. The number of neurons tested is given at the bottom of the open column. " $p<.01,{ }^{* *} p<.001$, using a two-tailed paired Student's t-test, comparing prior to and following clonidine administration.

Table 1. Effect of Long-Term Drug Treatments with Befloxatone, Nisoxetine, or Paroxetine on the Late Excitatory Effect of the Firing Activity of Dorsal Hippocampus Pyramidal Neurons Induced by the Electrical Stimulation Before and After the Intravenous Administration of Clonidines

\begin{tabular}{|c|c|c|c|c|}
\hline \multirow[b]{2}{*}{ Treatment ${ }^{b}$} & \multirow{2}{*}{$\begin{array}{c}\text { Before } \\
\text { Clonidine }\end{array}$} & \multicolumn{2}{|c|}{ After Clonidine } & \multirow{2}{*}{$\begin{array}{c}\text { No. of } \\
\text { Neurons }\end{array}$} \\
\hline & & $10 \mu \mathrm{g} / \mathrm{kg}$ & $400 \mu \mathrm{g} / \mathrm{kg}$ & \\
\hline $\begin{array}{l}\text { Saline } \\
\text { Befloxatone } \\
\text { 6-OHDA }{ }^{\mathrm{d}}+\text { befloxatone } \\
\text { Nisoxetine } \\
\text { Paroxetine }\end{array}$ & $\begin{array}{r}102 \pm 25 \\
89 \pm 22 \\
135 \pm 37 \\
130 \pm 54 \\
172 \pm 69\end{array}$ & $\begin{array}{r}97 \pm 23 \\
117 \pm 15 \\
171 \pm 34 \\
131 \pm 35 \\
134 \pm 37\end{array}$ & $\begin{array}{l}140 \pm 25 \\
167 \pm 61 \\
162 \pm 42 \\
155 \pm 42 \\
123 \pm 36\end{array}$ & $\begin{array}{r}11 \\
9 \\
6 \\
9 \\
8\end{array}$ \\
\hline
\end{tabular}

"One neuron per rat was : :udied before and after the administration of clonidine.

b Befloxatone: $0.75 \mathrm{mg} / \mathrm{kg}$ per day; nisoxetine and paroxetine: $10 \mathrm{mg} / \mathrm{kg}$ per day.

c Data are expressed as the relative activation in milliseconds $t$ the standard error of the mean.

d One-hundred twenty micrograms free base of 6-OHDA was injected intracerebroventricularly 1 week before the initiation of the befloxatone treatment. 
Prior to
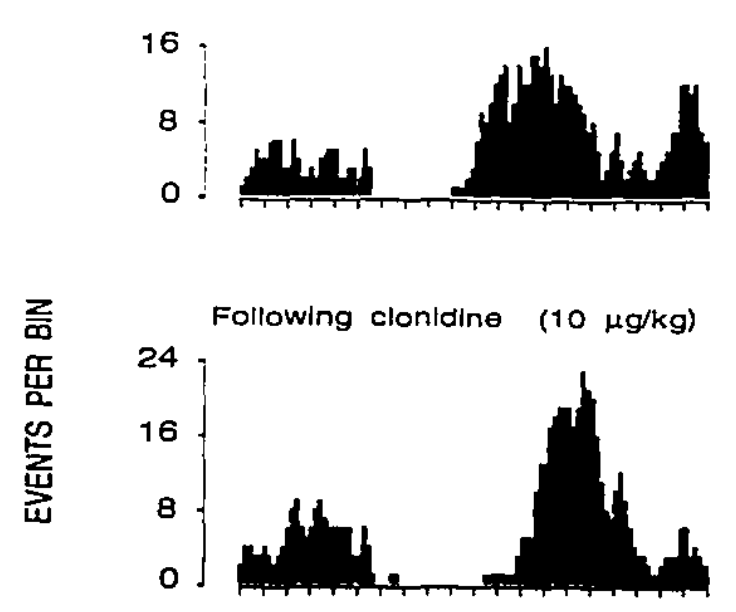

Following clonidine $(400 \mu g / k g)$

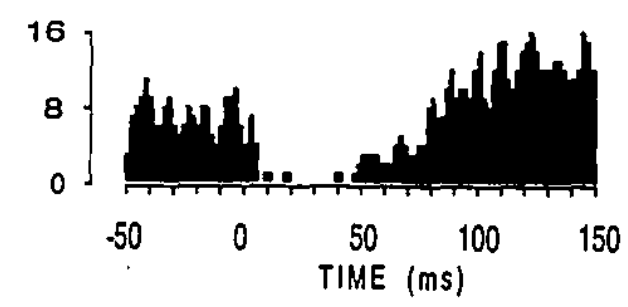

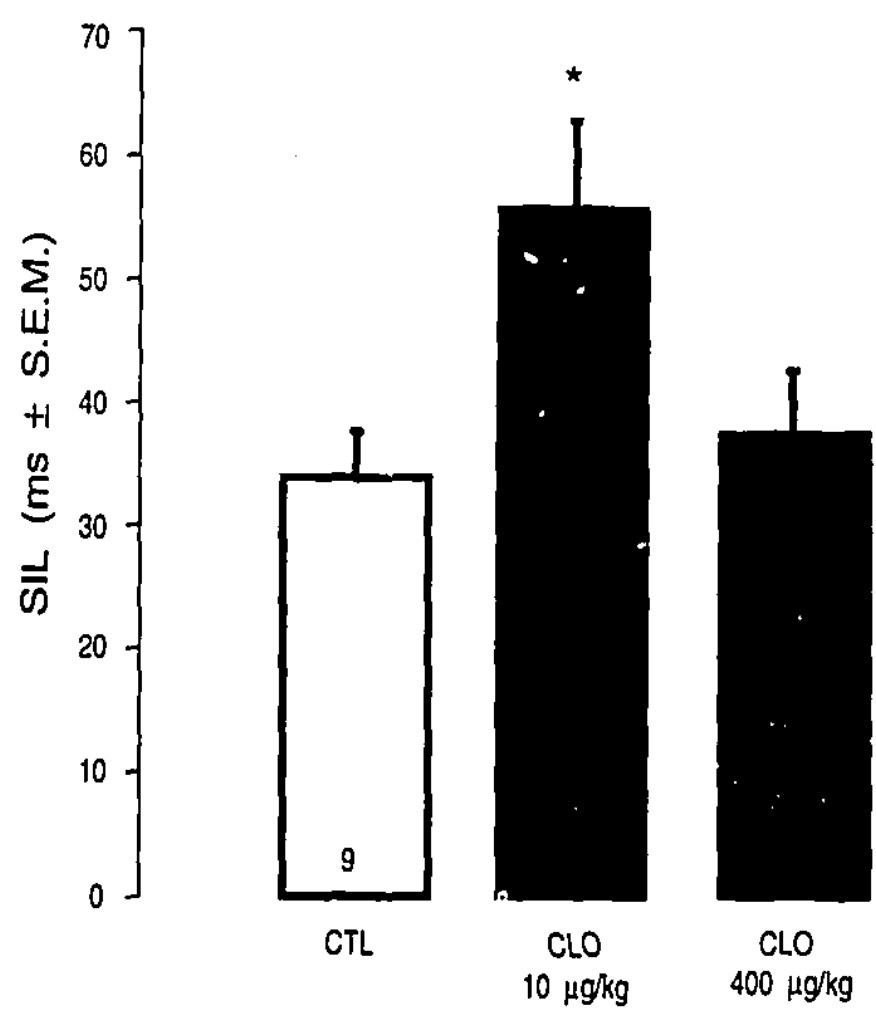

Figure 2. Effect of systemic injections of clonidine on the efficacy of the electrical stimulation of the ascending 5-HT pathway after a long-term treatment with $0.75 \mathrm{mg} / \mathrm{kg}$ per day of befloxatone. The minipumps containing the drug were removed 48 hours before the experiment began. A: Representative peristimulus time histograms illustrating the effects of clonidine $(10 \mu \mathrm{g} / \mathrm{kg}$ and $400 \mu \mathrm{g} / \mathrm{kg} \mathrm{IV})$ in a single experiment. B: Histograms showing the effects of the high and the low dose of clonidine in befloxatone-treated rats. The number of neurons tested is given at the bottom of the open column. $p<.01$, using a two-tailed paired Student's t-test, comparing prior to and following clonidine administration.

the experiments began to regain full enzymatic activity (Curet et al. 1992a). The results in Figure $2 \mathrm{~A}$ are representative peristimulus time histograms of the effect of clonidine in befloxatone-treated rats. The small dose of clonidine $(10 \mu \mathrm{g} / \mathrm{kg}$ IV) produced a $72 \% \pm 21 \%$ increase of the duration of the suppression of fring in the befloxatone-treated group (Fig. 2B). Although the amplitude of the effect of the small dose of clonidine appeared greater than that of the saline group $(37 \% \pm 8 \%$; Fig. 1B), the difference between the two groups did not reach statistical significance using covariance analysis. The subsequent administration of the high dose of clonidine $(400 \mu \mathrm{g} / \mathrm{kg}$ IV) decreased the duration of suppression of firing (Fig. 2B); however, unlike the controls (Fig. $1 B)$, it was not reduced below the initial value. This decreased responsiveness of the $\alpha_{2}$-adrenoceptors mediating the effect of the high dose of clonidine was not accompanied by any change in the responsiveness of pyramidal neurons of the $\mathrm{CA}_{3}$ region of hippocampus to microiontophoretic applications of 5-HT and NE. The suppressant effects of the microiontophoretic applications of 5-HT and NE on the firing rate of the pyramidal cells after the befloxatone treatment (5-HT: $322 \pm$ 37 spikes/nA, $n=13$; NE: $407 \pm 43$ spikes $/ n A ; n=13$ ) were not different from those of the saline-treated group (5-HT: $376 \pm 62$ spikes/nA, $n=11 ;$ NE: $400 \pm 71$ spikes/nA, $n=11$ ).

The assumption that the increased synaptic concentration of NE, but not that of 5-HT, is responsible for the decrease in the responsiveness of the $\alpha_{2}$-adrenoceptors mediating the effect of the high dose of clonidine was assessed by investigating the effect of the same befloxatone treatment in NE-denervated rats. Intracerebroventricular injections of the neurotoxin 6-OHDA (1 

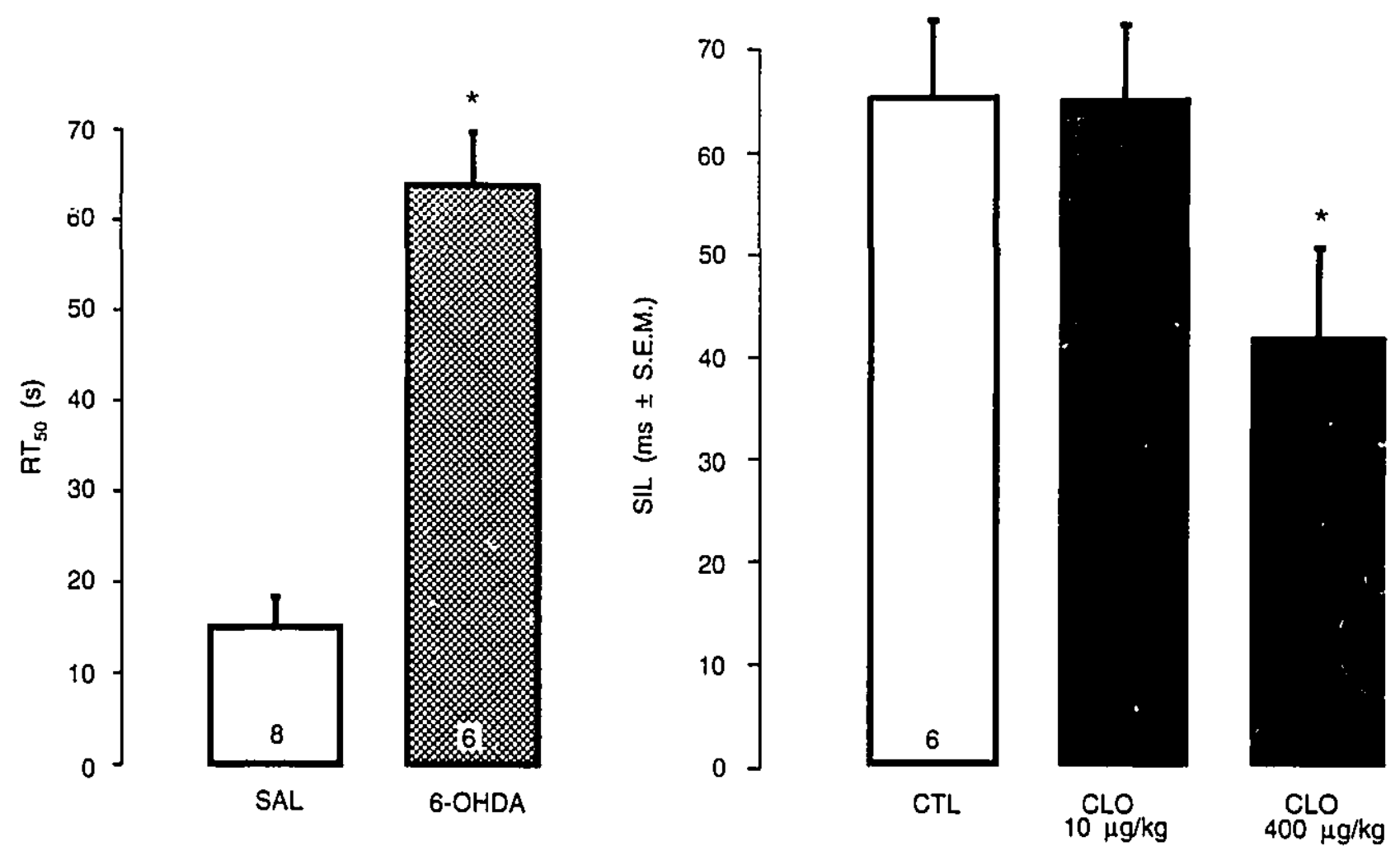

Figure 3. Effect of long-term treatment with $0.75 \mathrm{mg} / \mathrm{kg}$ per day of befloxatone in NE-denervated rats. The intracerebroventricular injection of $120 \mu \mathrm{g}$ of $6-\mathrm{OHDA}$ was given 1 week before the minipump containing the drug was installed. The minipumps were removed 48 hours before the experiment began. A: Neuronal recovery from the microiontophoretic application of $\mathrm{NE}$ in saline (SAL) and 6-OHDA + befloxatone-pretreated rats. The $\mathrm{RT}_{50}$ value is the time required for the neuron to recover its firing activity by $50 \%$ from the end of the application. The number of neurons tested is given at the bottom of each column. Data are expressed as mean \pm SEM. ${ }^{*} p<.001$, two-tailed Student's $t$-test comparing contril and 6-OHDApretreated rats. $\mathbf{b}$ : Histograms showing the effects of systemic injections of clonidine on the efficacy of the electrical stimulation of the ascending 5-HT pathway after a long-term treatment with befloxatone in six 6-OHDA-pretreated rats. ${ }^{*} p<.01$, using a two-tailed paired Student's t-test, comparing prior to and following clonidine administration.

hour after a pretreatment with the 5-HT reuptake inhibitor fluoxetine) were performed 1 week before initiating the befloxatone treatment. The effectiveness of the lesions was verified by assessing the $\mathrm{RT}_{50}$ of the firing rate of $\mathrm{CA}_{3}$ pyramidal neurons following the microiontophoretic application of NE. In keeping with the notion that intact $N E$ fibers are required for the NE reuptake process to allow a prompt recovery of the firing activity of these neurons (de Montigny et al. 1980), the marked increased $(327 \%)$ in the $\mathrm{RT}_{50}$ of $6-\mathrm{OHDA}-$ treated rats (Fig. $3 \mathrm{~A}$ ) confirmed an adequate lesioning of the NE terminals (except in two rats that were excluded from statistical analyses). In these conditions, the enhancing effect of the small dose of clonidine (10 $\mu g / \mathrm{kg}$ IV) was completely abolished, as previously observed (Mongeau et al. 1993). On the other hand, the decreasing effect of the high dose of clonidine $(400 \mu \mathrm{g} / \mathrm{kg}$
IV) was still present and was even of a greater amplitude than that in the saline group (saline: $25 \% \pm 5 \%$, $n=11 ; 6-$ OHDA: $39 \% \pm 9 \%, n=6 ; p=.06$, using covariance analysis).

The efficacy of the stimulation of the 5-HT pathway in the saline group was compared to those in the drug. treated groups using the unpaired Student's $t$ test. The diciation of suppression of firing in the 6-OHDA plus befloxatone group was increased by $71 \%$ (SIL values: saline $38 \mathrm{msec} \pm 3 \mathrm{msec}, n=11 ; 6-$ OHDA + befloxatone $65 \mathrm{msec} \pm 7 \mathrm{msec}, n=6 ; p<.01$; Figs. 1 and 3). However, the befloxatone treatment did not by itself change the efficacy of the stimulation in suppressing the furing activity of the pyramidal neurono (Figs. 1 and 2). It is noteworthy that in the two rats pretreated with 6-OHDA plus befloxatone for which the NE denervation failed (as indicated by the unchanged $\mathrm{RT}_{50}$ ), the 
increase in 5-HT transmission was not observed, and the effects of clonidine were similar to those observed for the befloxatone group (data not shown). Finally, none of the above treatments significantly changed the late excitatory effect on the firing rate induced by the electrical stimulation (Table 1).

Effect of Long-Term Monoanine Uptake Inhibition on the Modulation of 5-HT Neurotransmission by Clonidine

For two reasons, it was deemed crucial to test whether monoamine reuptake inhibitors could also affect the $\alpha_{2}$-adrenoceptors that modulate 5 -HT transmission. First, selective 5-HT reuptake inhibitors have long betn known to have antidepressent efficacy, and, second, 5 - $\mathrm{HT}$ and NE reuptake inhibitors have been shown to increase the synaptic concentration of 5-HT and NE, respectively (L'Heureux et al. 1986; Bel and Artigas 1992; Curet et al. 1992b). The effect of a sustained increase in the synaptic concentration of either NE or 5-HT was investigated using specific reuptake inhibitors. Following long-term treatment with the selective 5-HT reuptake inhibitor paroxetine $(10 \mathrm{mg} / \mathrm{kg}$ per day for 21 days), the effects of the low and the high dose of clonidine on 5-HT transmission were unchanged. After the paroxetine treatment, the small dose of clonidine (10 $\mu \mathrm{g} / \mathrm{kg}$ IV) increased the efficacy of the stimulation of the $5-\mathrm{HT}$ pathway by $29 \% \pm 9 \%$ whereas the high dose $(400 \mu \mathrm{g} / \mathrm{kg}$ IV) decreased it by $25 \% \pm 7 \%$ (Fig. $4 \mathrm{~A}$ ).

In contrast, the 21-day treatment with the selective NE reuptake inhibitor nisoxetine $(10 \mathrm{mg} / \mathrm{kg}$ per day) produced effects similar to those obtained in the befloxatone-treated rats. The small dose of clonidine produced a $30 \% \pm 8 \%$ increase (Fig. $4 \mathrm{~B}$ ), which was similar to that of the saline group (Fig. 1B). The subsequent administration of the high dose of clonidine decreased the duration of suppression of firing; however, it was not reduced below the initial value (Fig. 4B). Furthermore, as observed in the befloxatone group (Fig. $2 B)$, there was no change in the responsiveness of pyramidal neurons of the $\mathrm{CA}_{3}$ region of hippocampus to microiontophoretic applications of 5-HT and NE in the nisoxetine-treated rats. The suppressant effects of the microiontophoretic applications of 5-HT and NE on the firing rate of the pyramidal neurons after the nisoxetine treatment (5-HT: $332 \pm 66$ spikes/nA, $n=11$; NE: $378 \pm 71$ spikes $/ n A, n=11$ ) were not different from those of the saline-treated group (5-HT: $376 \pm 62$ spikes/nA, $n=11$; NE: $400 \pm 71$ spikes/nA, $n=11$ ).

The paroxetine and nisoxetine treatments did not significantly modify the efficacy of the stimulation of the 5-HT pathway in suppressing the fring activity of pyramidal neurons of the dorsal hippocampus (Fig. 4). In addition, the late excitatory effect of the stimulation
A

\section{PAROXETINE}

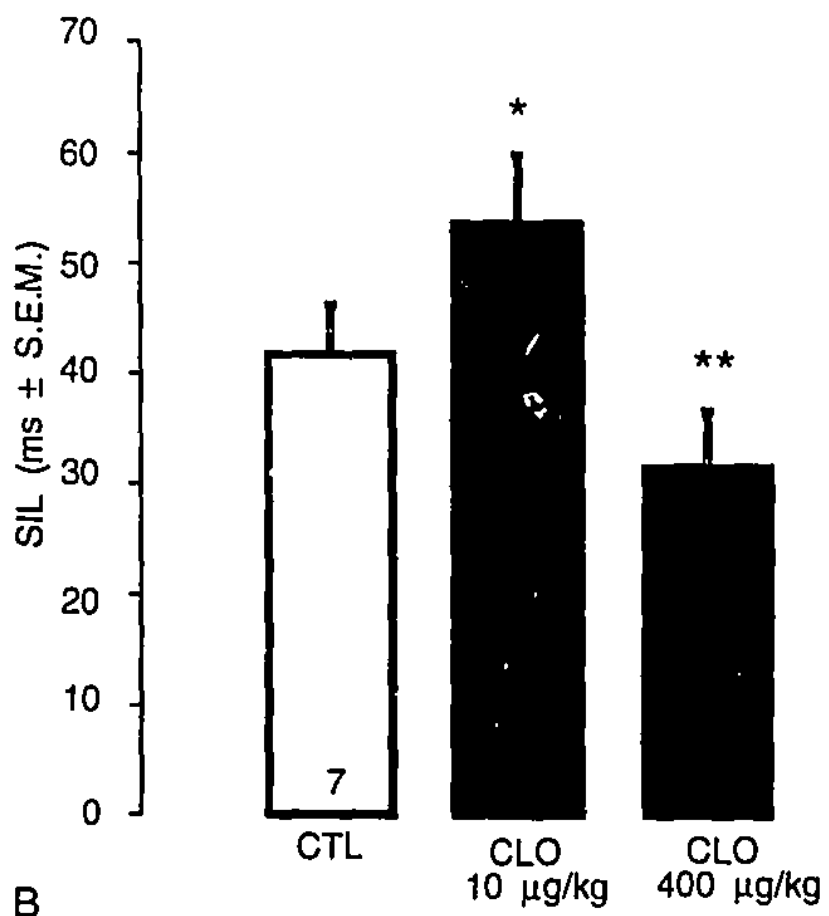

NISOXETINE
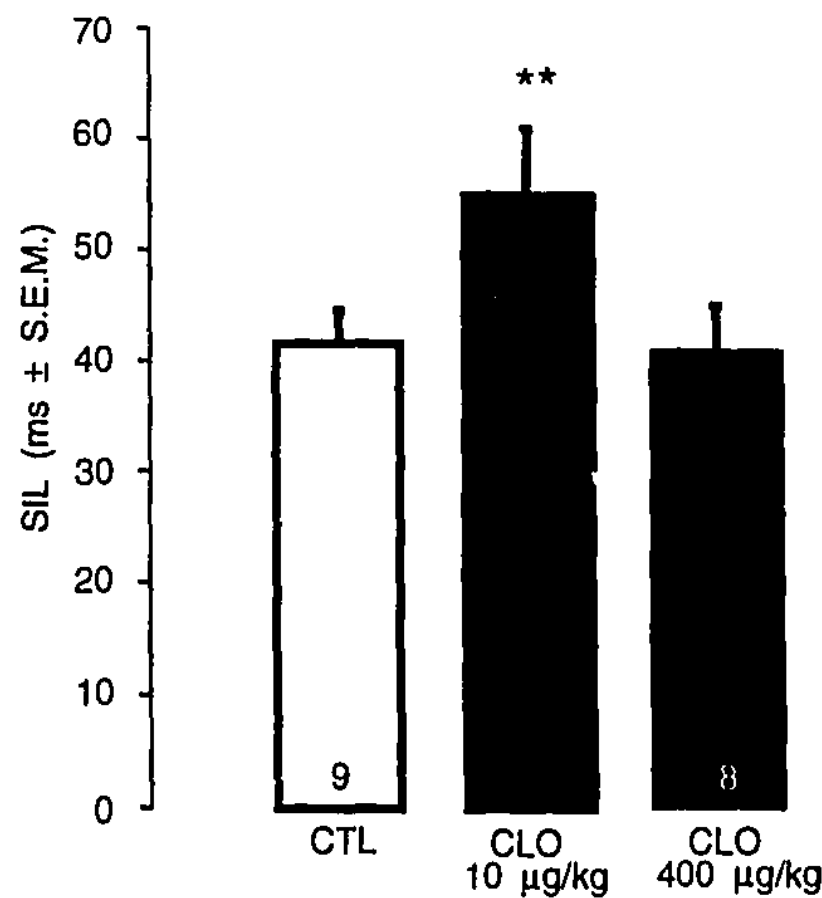

Figure 4. Histograms showing the effect of systemic injections of clonidine on the efficacy of the electrical stimulation of the ascending 5-HT pathway after a long-term treatment with $10 \mathrm{mg} / \mathrm{kg}$ per day of paroxetine (A) and $10 \mathrm{mg} / \mathrm{kg}$ per day of nisoxetine (B). The number of rats tested is given inside the columns. " $p<.05,{ }^{* *} p<.01$ using a two-tailed paired Student's $t$-test, comparing prior to and following clonidine administration. 
observed on these same neurons was not changed by these treatments (Table 1 ).

\section{DISCUSSION}

The present electrophysiologic data show that longterm treatment with pharmacologic agents that increase the synaptic concentration of NE, but not that of 5-HT, decrease the inhibitory action of a high dose of clonidine $(400 \mu \mathrm{g} / \mathrm{kg}$ IV) on 5-HT neurotransmission and that the enhancing effect of a low dose of clonidine $(10 \mu \mathrm{g} / \mathrm{kg}$ IV) is not affected by such treatments (Figs. 2, and 4B). These changes observed in the befloxatone and the nisoxetine groups are most likely related to the sustained increase in NE concentration in the synapse, because similar changes were not produced either by longterm 5-HT reuptake blockade or by long-term MAO-A inhibition in NE-denervated rats (Figs. 3B and 4B). None of the long-term treatments produced any significant changes in the enhancing effect of the low dose of clonidine (Figs. 2 and 4). However, the lesion of the NE neurons abolished, as expected, this enhancing effect of clonidine (Fig. 3B).

In a previous study, the incremental and decremental effects of clonidine have been fully characterized using the present electrophysiologic paradigm (Mongeau et al. 1993). On the one hand, it was concluded that the reducing effect of the high dose of clonidine is most likely mediated by the activacion of $\alpha_{2}$-adrenergic heteroreceptors on 5-HT terminals, which results in a decrease in the amount of 5-HT released per action potential. On the other hand, low doses of clonidine enhance the suppression of firing by preferentially activating $\alpha_{2}$-adrenergic autoreceptors, thereby reducing the concentration of endogenous NE tonically activating $\alpha_{2}$-adrenergic heteroreceptors on 5-HT terminals (Mongeau et al. 1993). Thus, the present results suggest that although the sensitivity of $\alpha_{2}$-adrenergic heteroreceptors is reduced by long-term treatment with nisoxetine and befloxatone, an inhibitory action of endogenous NE on 5-HT neurotransmission still appears to be present.

The magnitude of the effect of the small dose of clonidine on 5-HT neurotransmission does not provide a direct estimate of the responsiveness of the $\alpha_{2}-$ adrenergic autoreceptors, because its magnitude is also determined by the level of activation of $\alpha_{2}$-adrenergic heteroreceptors by endogenous NE. Thus, the unaltered effect of the low dose of clonidine after these treatments is not an indication that the $\alpha_{2}$-adrenergic autoreceptors on the cell body and terminals of NE neurons are normosensitive. Previous investigations performed in our laboratory have shown that terminal $\alpha_{2}$ adrenergic autoreceptors are desensitized following a long-term treatment with the NE reuptake inhibitor desipramine (Lacroix et al. 1991). However, despite an attenuated responsiveness of the somatodendritic $N E$ autoreceptors of locus coeruleus neurons to clonidine following this treatment, a $10-\mu \mathrm{g} / \mathrm{kg}$ IV dose of clonidine still substantially decreased $(85 \%$ instead of $100 \%)$ the furing rate of these neurons. It can thus be concluded that the desensitization of the $\alpha_{2}$-adrenergic autoreceptors would not hinder the enhancement of 5-HT neurotransmission that results from a decrease in the synaptic concentration of NE when these autoreceptors are activated by the $10-\mu \mathrm{g} / \mathrm{kg}$ IV dose of clonidine. In fact, a desensitization of $\alpha_{2}$-adrenergic autoreceptors could even increase the inhibitory tone of NE on 5-HT neurotransmission, because the synaptic concentration of $\mathrm{NE}$ is normally expected to be increased when the negative feedback autoregulation is decreased.

It is also interesting that the high dose of clonidine in both nisoxetine- and befloxatone-treated rats brought back the effectiveness of the stimulation to initial value (Figs. 2B and $4 B$ ), suggesting that these receptors still possess residual capacity to modulate $5-\mathrm{HT}$ release after the befloxatone or the nisoxetine treatment. The tonic inhibitory action of endogenous NE on the $\alpha_{2}$ adrenergic heteroreceptors at baseline was probably maximal because the high dose of clonidine merely reestablished the initial duration of suppression of firing (Figs. 2B and 4B).

These observations lead us to the following interpretation: normally $\mathbf{5}-\mathrm{HT}$ neurotransmission is decreased in the presence of an increased NE output. After long-term exposure to a high concentration of synaptic $\mathrm{NE}$, there is a desensitization of $\alpha_{2}$-adrenergic heteroreceptors. Although the paradigm used in the present study does not provide clues about the cellular mechanisms involved, it might result from a decrease in the density of the receptors, a change in the coupling of the receptors with their $G$ proteins, or an alteration in second messenger function. This desensitization would hinder the tonic inhibitory action of the NE system on 5-HT neurotransmission, more so in the condition of a high NE output.

No change in the efficacy of 5-HT neurotransmission was observed with long-term treatment with befloxatone, paroxetine, or nisoxetine. However, in the 6-OHDApretreated rats, the long-term befloxatone treatment significantly increased the duration of suppression of fring. This increase most likely resulted from the depletion of the endogenous NE that would normally exert a tonic inhibitory action on 5-HT neurotransmission through $\alpha_{2}$-adrenergic heteroreceptors. Finally, the increase in the magnitude of the effect of the high dose of clonidine in these rats was expected and is consistent with our previous study (Mongeau et al. 1993). It was most likely related to a supersensitivity of the $\alpha_{2}$-adrenergic heteroreceptors resulting from the NE depletion. 
To our knowledge, no study has directly assessed the modulation of 5-HT neurotransmission by $a_{2}$-adrenergic ligands following antidepressant treatments in an in vivo paradigm. Nevertheless, this has been previously investigated in in vitro studies. Some have failed to detect a reduction in the efficacy of $\alpha_{2}$-adrenergic heteroreceptors function following MAO inhibition of $\mathrm{NE}$ reuptake blockade. Groß et al. (1987) reported no change in the potency of clonidine to inhibit the electrically evoked overflow of $\left[{ }^{3} \mathrm{H}\right] 5-\mathrm{HT}$ in preloaded rat brain cerebral cortex slices when superfusing throughout the experiment $1 \mu \mathrm{M}$ of the NE reuptake inhibitor viloxazine. This result suggested that short-term exposure to an elevated synaptic concentration of NE does not desensitize $\alpha_{2}$-adrenergic heteroreceptors on $5-\mathrm{HT}$ terminals. However, long-term NE reuptake blockade with desipramine or long-term MAO inhibition with MDL 72394 or pargyline has not been reported to change the ability of $\alpha_{2}$-adrenergic heteroreceptors to reduce the electrically induced release of $\left[{ }^{3} \mathrm{H}\right] 5-\mathrm{HT}$ in rat cortical and hippocampal slices (Schlicker et al. 1982; Schoffelmeer and Mulder 1982; Palfreyman et al. 1986). Finally, another study using rat brain synaptosomes revealed that the attenuation of $\mathrm{K}^{+}$-stimulated release of $\left[{ }^{3} \mathrm{H}\right] 5-\mathrm{HT}$ by clonidine was diminished following a long-term treatment with the irreversible MAO inhibitor clorgyline but not by desipramine (Ellison and Campbell 1986). This effect of clorgyline was, however, attributable to a competition between clonidine and the increased NE in the biophase. Such a competition, however, cannot account for the decreased sensitivity of $\alpha_{2}$-adrenergic heteroreceptors to the high dose of clonidine observed in the pres.'nt study after the befloxatone treatment, because this drug is a reversible MAO inhibitor and the minipumps were removed 48 hours before the experiments. The method of administration of the drugs might explain the absence of desensitization of $\alpha_{2}$-adrenergic heteroreceptors in the abovementioned studies. In the present study, the drugs were continuously delivered through osmotic minipumps inserted subcutaneously, whereas desipramine was administered orally in the studies by Schlicker et al. (1982) and Ellison and Campbell (1986) and iritraperitoneally in the study of Schoffelmeer and Mulder (1982). The oral or intraperitoneal administration of desipramine in rats might not produce sustained reuptake blockade, considering the important furst-pass hepatic metabolism and also the much faster catabolism of such drugs in rats than in humans.

In vitro studies performed in our laboratory using osmotic minipumps provided results consistent with the desensitization of $\alpha_{2}$-adrenergic heteroreceptors. long-term treatment with minalcipran, a NE reuptake nhibitor, reduced the NE inhibition of the electrically induced release of $\left[{ }^{3} \mathrm{H}\right] 5-\mathrm{HT}$ in the rat hippocampus, whereas long-term treatment with befloxatone shifted the concentration-effect curve to the right of the $\alpha_{2-}$ adrenergic agonist UK 14.304 on electrically induced release of $\left[{ }^{3} \mathrm{H}\right] 5-\mathrm{HT}$ in guinea pig hypothalamus slices (Blier and Bouchard 1992; Blier et al. 1993). Hence, these results suggest that $\alpha_{2}$-adrenergic heteroreceptors are endowed with the capacity to become desensitized after a sustained increase in NE synaptic concentration.

Although the antidepressant property of befloxatone has not yet been documented in humans, all potent MAO-A inhibitors, specific or not, that bind irreversibly or not to the enzyme (clorgyline, phenelzine, tranylcypromine, moclobemide, brofaromine, and $t r$ oxatone) increase 5-HT and NE synaptic concentra : in and are effective in major depression. Furthermore, minalcipran which, like nisoxetine, selectively blocks the reuptake of NE (Blier et al. 1993), has been reported to be an effective antidepressant in humans (Macher et al. 1989; von Frenckell et al. 1990). It is thus possible that the desensitization of $\alpha_{2}$-adrenergic heteroreceptors on 5-HT terminals contributes to the therapeutic action of these drugs.

\section{ACKNOWLEDGMENTS}

This work was supported in part by the Medical Research Council of Canada (MRC) Grants MT.6144 and 11014 and the Fonds de la Recherche en Santé du Québec (FRSQ). R. Mongeau is the recipient of a studentship from FRSQ and $P$. Blier is the recipient of a scholarship from MRC.

\section{REFERENCES}

Bel N, Artigas F (1992): Fluvoxamine preferentially increases extracellular 5-hydroxytryptamine in the raphe nuclei: An in vivo microdialysis study. Eur J Pharmacol 229: 101-103

Blier P, de Montigny C (1983): Electrophysiological investigations on the effect of repeated zimelidine administration on serotonergic neurotransmission in the rat. J Neurosci 3:1270-1278.

Blier P, de Montigny C (1985): Short-term lithium administration enhances serotonergic neurotransmission: Electrophysiological evidence in the rat CNS. Eur J Pharmacol 113:69-77

Blier P, Chaput Y, de Montigny C (1988): Long-term 5-HT reuptake blockade, but not monoamine oxidase inhibition, decreases the function of terminal 5-HT autoreceptors: An electrophysiological study in the rat brain. NaunynSchmiedebergs Arch Pharmacol 337:246-254

Blier P, de Mlontigny C, Chaput Y (1990): A role for the serotonin system in the mechanism of action of antidepressant treatments: Preclinical evidence. J Clin Psychiatry 51 (Suppl): 14-20.

Blier P, Bouchard C (1992): Enhancement of serotonin release in the guinea pig hypothalamus following long-term 
treatment with antidepressant drugs. Clin Neurophar macol 13 (Suppl 1): 66B

Blier P. Mongeau R, Weiss M, de Montigny C (1994): Modulation of serotonin neurotransmission by presynaptic $\alpha_{2-}$ adrenergic receptors: $A$ target for antidepressant pharmacotherapy? Internat! Acad Biomed Drug Res (In press)

Chaput Y, de Montigny C, Blier P (1986): Effects of a selective 5-HT reuptake blocker, citalopram, on the sensitivity of 5.HT autoreceptors: Electrophysiological studies in the rat brain. Naunyn-Schmiedebergs Arch Pharmacol 333:342-348

Chaput Y, de Montigny C (1988): Effects of the 5-hydroxytryptamine receptor antagonist, BMY 7378, on 5-hydroxy. tryptamine neurotransmission: Electrophysiological studies in the rat central nervous system. J Pharmacol Exp Ther 246:359-370

Crews FT, Smith CB (1978): Presynaptic alpha-receptor subsensitivity after long-term antidepressant treatment. Science 202:322-324

Cohen RM, Ebstein RP, Daly JW, Murphy DL (1982): Chronic effects of a monoamine uxidase inhibiting antidepressant: Decreases in functional alpha-adrenergic autoreceptors precede the decrease in norepinephrine-stimulated cy. clic adenosine $3^{\prime}: 5^{\prime}$-monophosphate systems in rat brain. J Neurosci 2:1588-1595

Curet O, Damoiseau G, Aubin N (1992a): Biochemical profile of befloxatone, a new reversible MAO-A inhibitor. Clin Neuropharmacol 15 (Suppl 1):424b

Ciret O, de Montigny C, Blier P (1992b): Effect of desipramine and amphetamine on noradrenergic neurotransmission: Electrophysiological studies in the rat brain. Eur J Pharmacol 221:59-70

de Montigny C, Wang RY, Reader TA, Aghajanian GK(1980): Monoaminergic denervation of the rat hippocampus: Microiontophoretic studies on pre- and postsynaptic supersensitivity to norepinephrine and serotonin. Brain Res 200:363-376

Doyle MC, George AJ, Ravindran AV, Phillpot R(1985): Flatelet $\alpha_{2}$-adrenoceptor binding in elderly depressed patients. Am J Psychiatry 142:1489-1490

Ellison DW, Campbell IC (1986): Studies on the role of $\alpha_{2}-$ adrenoceptors in the control of synaptosomal $\left[{ }^{3} \mathrm{H}\right] 5$ hydroxytryptamine release: Effects of antidepressant drugs. J Neurochem 46:218-223

Feuerstein TJ, Mutschler A, Lupp A, van Velthoven V, Schlicker E, Gothert M (1993): Endogenous noradrenaline activates $\alpha_{2}$-adrenoceptors on serotonergic nerve endings in human and rat neocortex. J Neurochem $61: 474-480$

Finberg JPM, Tal A (1985): Reduced peripheral presynaptic adrenoceptor sensitivity following chronic antidepressant treatment in rats. Br J Pharmacol 84:609-617

Frankhuyzen AL, Mulder A (1980): Noradrenaline inhibits depolarization-induced $\left[{ }^{3} \mathrm{H}\right]$-serotonin release from slices of rat hippocampus. Eur J Pharmacol 63:179-182

Frankhuyzen AL, Mulder AH (1982): Pharmacological characterization of presynaptic $\alpha$-adrenoceptors modulating $\left[{ }^{3} \mathrm{H}\right] 5$-hydroxytryptamine release from slices of the hippocampus of the rat. Eur J Pharmacol 81:97-106

Fuller RW, Snoddy HD, Perry KW (1979): Nisoxetine an- tagonism of norepinephrine depletion in brain and heart after alpha-methyl-m-tyrosine administration. Neuropharmacology 18:76\% -770

Galzin AM, Poirier MF, Lista A, Chodkiewicz JP, Blier P, Ramdine R, Lóo H, Roux FX, Redondo A, Langer SZ (1992): Characterization of the 5-hydroxytryptamine receptor modulating the release of $5-\left[{ }^{3} \mathrm{H}\right]$ hydroxytryptamine in slices of the human neocortex. J Neurochem 59:1293-1301

Garcia-Sevilla JA, Guimon J, Garcia-Vallejo, Fuster MJ (1986): Biochemical and functional evidence of supersensitive platelet alpha-2 adrenoceptor in major affective disorder. Arch Gen Psychiatry 43:51-57

Garcia-Sevilla JA, Udina C, Fuster Mj, Alvarez E, Cassas M (1987): Enhanced binding of ${ }^{3} \mathrm{H}(-)$ adrenaline to platelets of depressed patients with melancholia: Effect of longterm clomipramine treatment. Acta Psychiatr Scand $75: 150-157$

Göthert M, Huth H (1980): Alpha-adrenoceptor-mediated modulation of 5-hydroxytryptamine release from rat brain cortex slices. Naunyn-Schmiedebergs Arch Pharmacol 313:21-26

Gothert M, Huth H. Schlicker E (1981): Characterization of the receptor subtype involved in alpha-adrenoceptormediated modulation of serotonin release from rat brain cortex slices. Naunyn-Schmiedebergs Arch Pharmacol 317:199-203

Groß G, Hante K, Göthert M (1987): Effect of antidepressant and neuroleptic drugs on the electrically evoked release of serotonin from rat cerebral cortex. Psychopharmacology $91: 175-181$

Kandel ER, Spencer WA (1961): Electrophysiology of hippocampal neurons. II. After-potentials and repetitive fring. J Neurophysiol 24:243-259

Lacroix D, Blier P, Curet O, de Montigny (1991): Effects of long-term desipramine administration of adrenoceptors and norepinephrine reuptake: Electrophysiological studies in the rat brain. J Pharmacol Exp Ther 257:1081-1090

L'Heureux R, Dennis T, Curet O, Scatton B (1986): Measurement of endogenous noradrenaline release in the rat cerebral cortex in vivo by transcortical dialysis: Effects of drugs affecting noradrenergic transmission. J Neurochem 46:1794-1801

Macher JP, Schel JP, Serre C, von Frenckell R, Huck JC, Demarez JP (1989): Double-blind placebo-controlled study of minalcipran in hospitalized patients with major depres. sive disorders. Neuropsychobiology 22:77-82

Maura G, Gemignani A, Raiteri M (1982): Noradrenaline inhibits central serotonin release through alphaz-adrenoceptors located on serotonergic nerve terminals. NaunynSchmiedebergs Arch Pharmacol 320:272-274

McMillen BA, Warnack W, German DC, Shore PA (1980): Effects of chronic desipramine treatment on rat brain noradrenergic responses to alpha-adrenergic drugs. Eur J Pharmacol 61:239-246

Mongeau R, Blier P, de Montigny (1993): In vivo electrophysiological evidence for tonic activation by endogenous noradrenaline on $\alpha_{2}$-adrenergic heteroreceptors of 5-hydroxytryptamine terininals in the rat hippocampus. Naunyn-Schmiedebergs Arch Pharmacol 347:266-272

Palfreyman MG, Mir AK, Kubina M, Middlemiss DN, 
Richards M, Tricklebank MID, Fozard JR (1986): Monoamine receptor sensitivity changes following chronic administration of MDL 72394, a site-directed inhibitor of monnamine oxidase. Eur J Pharmacol 130:73-89

Piletz JE, Halaris A, Saran A, Marler M (1991): Desipramine lowers tritiated para-aminoclonidine binding in platelets of depressed patients. A.rch Gen Psychiatry 48:813-820

Schlicker E, Göthert M, Clausing R (1982): Acute or chronic changes of noradrenergic neurotransmission do not affect the $\alpha$-adrenuceptor-mediated inhibition of ${ }^{3} \mathrm{H}$-serotonin release in the cerebral cortex. Naunyn-Schmiedebergs Arch Pharmacol 320:38-44

Schoffelmeer ANM, Maulder AH (1982): ${ }^{3} \mathrm{H}$-Noradrenaline and ${ }^{3} \mathrm{H}-5$-hydroxytryptamine release from rat brain slices and its presynaptic alpha-adrenergic modulation after lcng-term desipramine pretreatment. Naunyn. Schmiedebergs Arch Pharmacol 318:173-1.90

Spyraky C, Fibiger HC (1980): Functional evidence for subsensitivity of noradrenergic $\alpha_{2}$-receptors after chronic desipramine treatment. Life Sci 47:1863-1867

Starke K, Montel H (1973): Involvement of alpha-receptors in clonidine-induced inhibition of transmitter release from central monoamine neurones. Neuropharmacology 12: 1073-1080

Svensson TH, Usdin T (1978): Feedback inhibition of brain noradrenaline neurons by tricyclic antidepressants: $\alpha-\mathrm{Re}$ ceptor mediation. Science 202:1089-1091

Takeda T, Harada T, Otsuki S (1989): Platelet ${ }^{3} \mathrm{H}$-clonidine and ${ }^{3} \mathrm{H}$-imipramine birıding and plasma cortisol level in depression. Biol Psychiatry 26:52 60

Thomas DK, Nelson DR, johnson AM (1987): Biochemical effects of the antidepressant paroxetine, a specific 5-hydroxytryptamine uptake inhibitor. Psycinopharmacology 93:193-200

von Frenckell R, Ansseau M, Serre C, Sutet P (1990): Pooling two coritrolled comparisons of milnacipran (F2207) and amitriptiline in endogenous inpatients. Intl Clin Psy. chopharmacol 5:49-56

Willner P (1985): Depression: A Psychobiological Synthesis. NY, Wiley.

Yocca FD, Hyslop DK, Smith DW, Maayani S (1987): BMY 7378, a buspirone analog with high affinity, selectivity, and low intrinsic activity at the $5-\mathrm{HT}_{1 \mathrm{~A}}$ receptor in rat and guinea pig hippocampus membranes. Eur J Pharmacol 137:293-294 


\section{Third article}

At this point, we were interested to known whether other types of antidepressant treatments would produce the desensitization of $\alpha_{2}$-adrenergic heteroreceptors on 5-HT fibers. The effect of repeated electroconvulsive shocks, a very effective treatment for depression, was studied with the electrophysiological paradigm using clonidine as in the preceding studies. There were suggestions in the literature indicating that some antidepressant drugs achieve therapeutical efficacy through the blockade of $\alpha_{2}$ adrenoceptors. We have thus deemed crucial to test drugs endowed with this property. Again, the responsiveness of postsynaptic $\alpha_{2}$-adrenoceptors and 5-HT1A receptors was assessed in parallel with microiontophoresis techniques. We have also decided to test the effect of the above antidepressant treatments on the sensitivity of terminal $5-\mathrm{HT}_{1 \mathrm{~B}}$ autoreceptors. In brief, this sensitivity is estimated by increasing the frequency of the electrical stimulation of the $5-\mathrm{HT}$ pathway, from $1 \mathrm{~Hz}$ to $5 \mathrm{~Hz}$. In this condition, there is a reduction in the duration of the suppression of firing that is explained by an increased activation of terminal autoreceptors consequent to the enhanced 5-HT output.

This article entitled "Effects of long-term alphaz-adrenergic antagonists and electroconvulsive treatments on the alpha2-adrenoceptors modulating serotonin neurotransmission" by myself, Claude de Montigny and Pierre Blier was published in the Journal of Pharmacology and Experimental Therapeutics (1994, vol. 269, pp. 11521159). 


\title{
Effects of Long-Term Alpha-2 Adrenergic Antagonists and Electroconvulsive Treatments on the Alpha-2 Adrenoceptors Modulating Serotonin Neurotransmission ${ }^{1}$
}

\author{
RAYMOND MONGEAU, ${ }^{2}$ CLAUde de MONTIGNY and PIERRE blieR ${ }^{3}$ \\ Department of Psychlatry, Nouroblological Psychiatry Unit, MCGill University, Montrad, Quebec, Canada H3R 1A1 \\ Accepted for publication February 21, 1994
}

\begin{abstract}
Previous results from our laboratory indicate that small doses of the alpha-2 adrenergic agonist clonidine increase serotonin (5-HT) nourotransmission by attenuating the release of endogenous norepinephrine (NE), as a result of the activation of alpha2 adrenergic autoreceptors on NE neurons, and that high doses decrease 5-HT neurotransmission by activating directly alpha-2 adrenergic heteroreceptors on 5-HT terminals. In addition, we have shown that long-term treatments with a monoamine oxjdase inhibitor or a selective NE, but not a 5-HT, reuptake inhibitor abolish the effect of a high dose of clonidine, but not that of a small dose of clonidine. The aim of the present study was to determine whether the alpha-2 adrenergic antagonists idazoxan $(10 \mathrm{mg} / \mathrm{kg} /$ day $\times 21$ days s.c.) and mianserin (5 mg/kg/day $x$ 21 days s.c.), or electroconvulsive shocks (6 or 7 over a 2-week period) would also affect the a/pha-2 adrenoceptors modulating 5-HT neurotransmission in the rat hippocampus. The responsiveness of these hetereceptors was tested in parallel with those of the terminal 5-HT, autorecoptors and of the postsynaptic 5-HT, a and alpha-2 adrenergic receptors. None of the above treatments altered the responsiveness of the $5-H T_{10}$ autorecep-
\end{abstract}

tors, as assessed by comparing the differential effectiveness of 1 and $5 \mathrm{~Hz}$ electrical stimulations of the 5-HT pathway. Idazoxan and mianserin did not affect the responsiveness of the postsynaptic 5-HT 1 and alpha-2 adrenergic receptors as indicated by the unchangeo suppressant effects of microiontophoretically applied 5-HT and NE. However, these alpha-2 adrenergic antagonists markedly decreased the reducing effect of $400 \mu \mathrm{g} / \mathrm{kg}$ i.v. of clonidine on the efficacy of the stimulation of the 5-HT pathway, indicating a desensitization of the alpha-2 adrenergic heteroreceptors on 5-HT terminals. Furthermore, the tonk inhibitory action of endogenous NE on these heteroreceptors was apparently weaker, because the enhancing effect of $10 \mu \mathrm{g} / \mathrm{kg} \mathrm{i.v.} \mathrm{of}$ clonidine also was reduced after long-term treatment with these two antagonists. In contrast, these above effects were not induced by repeated electroconvulsive shocks. Consistent with previous findings, the latter treatment increased the efficacy of the stimulation of the 5-HT pathway as we-l as the suppressant effect of microiontophoretically applied 5-HT, without altering that of NE.
5-HT terminals have long been known to be endowed with alpha-2 adrenergic heteroreceptors that modulate the evokedrelease of [ $\left.{ }^{3} \mathrm{H}\right]-5-H T$ (Starke and Montel, 1973; Frankhuijzen and Mulder, 1980, 1982; Göthert and Huth, 1980; Gothert et al, 1981; Maura et ah, 1982). By using an in vivo electrophysiological paradigm, we have shown recently that these alpha-2 adrenergic heteroreceptors in the rat hippocampus are activated tonically by endogenous NE (see fig. 1 and Mongeau et ah, 1993a). Small doses (2 and $10 \mu \mathrm{g} / \mathrm{kg}$ i.v.) of the alpho-2 adrenergic agonist clonidine enhances 5-HT neurotransmission, an effect which is abolished by an NE denervation and blocked by the alpha-2 adrenergic antagonist yohimbine. These results

Recoived for publication November 5, 1993.

This worts was wepported in pert by the Medical Research Council of Cened Granta MT-6144 and 11014 and the Fonds do in Recherche en Santi du Quibec.

Recipient of a Studentahip from the Medical Resench Council of Canad.

- Recipient of a Scbolanabip from the Medical Revench Council of Canadn. indicate that low doses of clonidine activate preferentially alpha-2 adrenergic autoreceptors on NE neurons, reducing the tonic inhibitory action of endogenous $\mathrm{NE}$ on alpha-2 adrenergic heteroreceptors on 5-HT terminals and consequently increasing 5-HT release. However, at higher doses (100 and $400 \mu \mathrm{g} / \mathrm{kg}$ i.v.), clonidine decreases 5-HT neurotransmission tirrough a direct activation of alpha-2 adrenergic heteroreceptors, because this effect is not affected by an NE denervation and is blocked by yohimbine (Mongeau et al, 1993).

In addition, we have shown that long-term treatments with a MAO inhibitor or a selective NE, but not a 5-HT, reuptake inhibitor abolish the effect of $\mathrm{a}$ high dose of clonidine, but not that of a small dose of clonidine (Mongeau et ah, 1994). It was thus concluded that long-term MAO or NE reuptake inhibition desensitize the alpha-2 adrenergic heteroreceptors. A sustained increase in NE synaptic availability most likely accounts for the desensitization of the alpha-2 adrenergic heteroreceptors, 


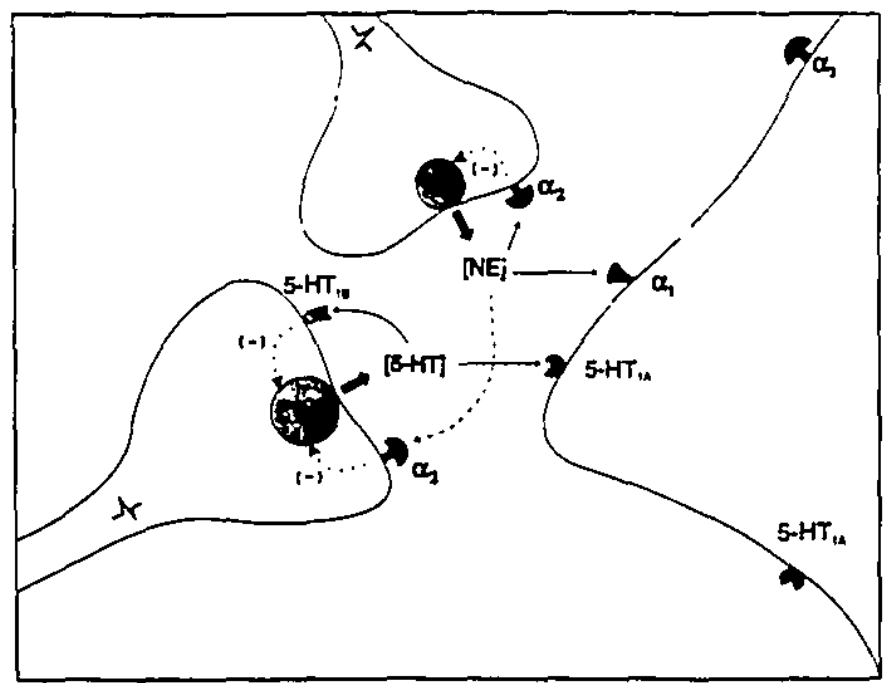

Fig. 1. Functional and anatomical relations of 5.HT and NE receptors. The effects on rat dorsal hippocampus pyramidal neurons of endogenous $\mathrm{NE}$ and 5-HT released by the electrical stimulation of the corresponding pathway are mediated by intrasynaptic alpha-1 adrenoceptors and 5HT 1 A receptors, respectively. Furthermore, the amount of NE and 5.HT released in the synaptic clett is negatively regulated by autoreceptors of the alpha-2 adrenerglc and 5-HT, subtypes. In contrast, NE and 5-HT applled by microiontophoresis activate extrasynaptic alpha-2 adrenoceptors and 5-HT, $1 \mathrm{a}$ receptors, respectively. Endogenous NE tonically inhibits 5-HT neurotransmission through alpha-2 adrenergic heteroreceptors located on 5-HT terminals.

inasmuch as long-term MAO inhibition fails to induce such a desensitization after an NE denervation (Mongeau et al., 1993b).

The aim of the present study was to determine whether other drugs acting on the NE system through different primary sites of action also would affect alpha. 2 adrenergic heteroreceptor function. An alternative way to modify NE neurotransmission was to block the alpha-2 adrenoceptors with two specific antagonists, idazoxan and $( \pm)$-mianserin (Bauman and Maitre, 1977; Doxey et al., 1983; Raiteri et al., 1983b). Studies using release from pretuaded brain slices, microdialysis or electrophysiological techniques have shown that these two alpha-2 adrenergic antagonists increase $\mathrm{NE}$ synaptic concentration in the rat brain in vitro, as well as in vivo when given acutely, by blocking the alpha-2 adrenergic autoreceptors (Rose et al., 1984; L'Heureux et al., 1986; Dennis et al., 1987; Curet and de Montigny, 1989). Furthermore, these drugs have antidepressant properties as for MAO inhibitors and monoamine reuptake blockers. Indeed, racemic ( \pm )-mianserin is used in Europe as an antidepressant drug (van Riezen et al., 1981), and there are reports supporting the antidepressant efficacy of idazoxan (Pinder and Sitsen, 1987; Osman et al., 1989; Taylor, 1989).

In the present study, the effects of long-term treatment with mianserin and idazoran were tested on the alpha.2 adrenoceptors that modulate 5-HT neurotransmission and compared with that of repeated ECS, which is generally considered as a "gold standard" in the treatment of depression. A well established effect of repeated ECS is the increase in 5-HT neurotransmission through the sensitization of postsynaptic $5 \cdot \mathrm{HT}_{34}$ receptors (de Montigny, 1984; Chaput et al, 1991). Endogenous NE release in the brain also is modulated by ECS (Thomas et al. 1992). Finally, the function of the 5-HT $\mathrm{T}_{1 \mathrm{~B}}$ autoreceptors and the postsynaptic $5-\mathrm{HT}_{1 \wedge}$ and alpha-2 adrenergic receptors (see fig. 1) was also assessed in parallel with that of the alpha-2 adrenoceptors that modulate 5.HT neurotransmission in order to determine the specificity of the changes induced by longterm alpha.2 adrenergic antagonism or repeated ECS.

\section{Materials and Methods}

Animals and treatments. Male Sprague-Dawley rats (Charles River. St. Constant, Qué, Can), maintained on a 12:12 light-dark cycle with free access to food and water, were treated for 3 weeks with either $5 \mathrm{mg} / \mathrm{kg} /$ day of mianserin, $10 \mathrm{mg} / \mathrm{kg} /$ day of idazoxan or saline by using osmo:ic minipumps (Alzet) implanted s.c. These minipumps were removed $48 \mathrm{hr}$ before the experiment in order to allow a complete elimination of the drugs. During a period of 2 weeks, group of rats was treated with siz or seven ECS under light halothane anesthesia. During the same period, controls received halothane without ECS.

Recording from dorsal hippocampus $\mathrm{CA}_{3}$ pyramidal neurons. Extracellular unitary recording of $\mathrm{CA}_{3}$ pyramidal neurons in the dorsal hippocampus and microiontophoresis applications were carried out with five-barreled micropipettes, pulled conventionally with the tip broken to a diameter of 9 to $11 \mu \mathrm{m}$. The central barrel, used for recording, was filled with a $2 \mathrm{M} \mathrm{NaCl}$ zolution and the side barrels contained the following solutions: 5 -HT creatinine sulfate $10.5 \mathrm{mM}$ in $200 \mathrm{mM} \mathrm{NaCl}, \mathrm{pH} 4), \mathrm{NE}$ bitartrate (20 $\mathrm{mM}$ in $200 \mathrm{mM} \mathrm{NaCl}, \mathrm{pH} 4)$, acetylcholine chloride $(20 \mathrm{mM}$ in $200 \mathrm{mM} \mathrm{NaCl}, \mathrm{pH}$ 4) and a $2 \mathrm{M} \mathrm{NaCl}$ solution used for automatic current balancing. The 5-HT and NE solutions were retained with a $-7 \mathrm{nA}$ current hetween ejections. Pyramidal neurons were identified by their large amplitude $(0.5-1.2 \mathrm{mV})$ and long duration $(0.8-1.2 \mathrm{msec})$ simple spikes alternating with complex spike discharges (Kandel and Spencer, 1961). These characteristics readily allow to differentiate pyramidal neurons from interneurons. A leak or a small current of acetylcholine $(-1$ to $5 \mathrm{nA})$ was used to activate silent or slowly discharging pyramidal neurons to a physiologic firing rate $(8-12 \mathrm{~Hz}$ ) (Ranck, 1975), because most of these cells do not discharge spontaneously in chloral hydrate-anesthetized rats. The responsiveness to microiontophoretic applicatior: of 5-HT and NE was evaluated from the number of spikes suppressed/nanoampere calculated on -line by computer analysis allowing a $100 \mathrm{msec}$ discrimination.

Stimulation of the 5-HT pathway. To activate the 5-HT projections to the dorsal hippocampus, a bipolar electrode (NE.100; David Kopf. Tujunga, $C A$ ) was implanted on the midline with \& $10^{\circ}$ backward angle in the ventromedial tegmentum, $1 \mathrm{~km}$ anterior to lambda and $8.3 \mathrm{~mm}$ below the cortical surface. Two hundred square pulses of 0.5 msec were delivered by a stimulator ( $\$ 8800$, Grass, Quincey, MA) at an intensity of $300 \mu \mathrm{A}$ and general!y at a frequency of $1 \mathrm{~Hz}$. Different frequencies of stimulation $(1$ and $5 \mathrm{~Hz}$ ) were used to evaluate the function of the terminal 5.HT autoreceptor. Thi. approach is based on the assumption that the higher the frequency of stımulation, the greater should be the degree of activation of the terminal autoreceptor at the time of arrival of the next stimulation-triggered action potential. Therefors, each pulse of the higher frequency of stimulation is expected to produce a smaller etfact on postsynaptic neurons, tach imoulse releasing a smaller amount of 5.HT. Evidence has been provided for the validity of this assumption (Chaput et al, 1986a). The stimulation pulses and the firing activity of the neurons recorded were fed to a computer equipped with a Tecmar interface. Peristimulus time histo. grams were generated to determine the duration of suppression of firing, measured in absolute silence value (in milliseconds). This pa. rameter is obtained by dividing the total number of events suppressed by the stimulation by the mean frequency of firing of the neuron recorded. It thus represents an estimate of the duration of the suppression of firing corrected for the mean prestimulation firing frequency. Several lines of evidence indicate that the effect of the electrical stimulation of the ascending 5.HT pathway is due to the release of 5 . HT into the synaptic cleft. First, it is virtually abolished by a pretreatment with the 5-HT neurotoxin, 5,7-dihydroxytryptamine (Blier and de Montigny, 1983, 1985). Second, it is blocked by the acute i.v. injection of the 5-HT 1 A receptor antagoniat BMY 7378 (Chaput and de 
Montigny, 1988). Third, it is enhanced by terminal 5.HT autoreceptor blockade (Chaput et al, 1986b). Fourth, it is reduced by terminal 5-HT autoreceptor activation (Chaput and de Montigny, 1988).

The effect of stimulating the ascending 5. HT fibers was determined while recording from the anme neuron before, and following, the successive i.v. injections of 10 and $400 \mu \mathrm{g} / \mathrm{kg}$ of clonidine. The time elapsed between the control stimulation periods and those following the administration of clonidine was of about 15 min.

Drugu. The following drugs were used: idazoran and mianserin (RBI, Natick, MA); 5-HT creatinine sulfate, NE bitartrate and acetylcholine chloride (Sigma Chemical Co., St. Louis, MO); and chloral hydrate (American Chemicals, Montreal, Québec, Canada),

Statistical analyeses. All results are expressed as means \pm S.E.M. The statistical significance of the difference between the effects of the stimulation of the 5.HT pathway at 1 and $5 \mathrm{~Hz}$, as well as before and after the acute administration of a drug, was assessed by using the paired Student's $t$ test. Possible differences in the magnitude of the effects of clonidine between control and treated rats were tested for statiatical significance by covariance analysis, the predrug value being used as the regressor. Differences in the efficacy of the stimulation of the 5-HT pathway and in the neuronal responsiveness to microiontophoretic applications of NE and 5-HT between control and treated rats were assessed for atatistical significance by using the unpaired Student's $f$ test.

\section{Results}

Effects of long-term alpha-2 adrenergic antagonist treatment. The suppressant effect of the electrical stimulation of the ascending 5-HT pathway on the firing rate of $\mathrm{CA}_{\text {, }}$ pyramidal neurons of the dorsal hippocampus was assessed after 3-week treatments with saline, idazoran $(10 \mathrm{mg} / \mathrm{kg} / \mathrm{day})$ or mianserin $(5 \mathrm{mg} / \mathrm{kg} / \mathrm{day})$ delivered through osmotic minipumps implanted s.c. These minipumps were removed $48 \mathrm{hr}$ before the experiment to allow elimination of the drugs. As shown in figure 2, the efficacy of the stimulation at 1 or $5 \mathrm{~Hz}$ was changed neither by the idazoran nor by the mianserin treatment. Furthermore, the magnitude of the reduction in the duration of firing recorded when the frequency of stimulation was increased from 1 to $5 \mathrm{~Hz}$ was not significantly different in the treated (idazoran, $-27 \pm 9 \%$ and mianserin, $-24 \pm 7 \%$ ) compared to the saline group $(-31 \pm 4 \%)$, thus indicating an unchanged responsiveness of the 5-HT $1 \mathrm{~B}$ autoreceptors.

The peristimulus time histograms of figure $3 \mathrm{~A}$ show that 10 $\mu \mathrm{g} / \mathrm{kg}$ of i.v. clonidine enhanced, whereas $400 \mu \mathrm{g} / \mathrm{kg}$ reduced, the duration of suppression of firing in the same neuron of a saline-treated rat. Similar results were obtained for control rats tested in parallel to the ECS treatment. In the pooled control groups (fig. 4), the administration of 10 and $400 \mu \mathrm{g} / \mathrm{kg}$ of i.v. clonidine increased by $33 \pm 8 \%$ and decreased by $24 \pm 5 \%$, respectively, the mean duration of suppression of firing when compared to the initial value. These incremental and decremental effects of clonidine were statistically significant at the 0.001 and 0.01 levels, respectively, by using the paired Student's $t$ test.

The duration of suppression of firing after 10 and $400 \mu \mathrm{g} / \mathrm{kg}$ i.v. of clonidine in the same neuron of an idazoran-treated rat is shown in the peristimulus time histograms of figure 3B. It shows that the low or the high dose of clonidine did not alter the efficacy of the stimulation in this case. On average, the 10 $\mu g / \mathrm{kg}$ i.v. dose of clonidine did not significantly increase the duration of suppression of firing in rats treated for 3 weeks with idazoran (fig. 5A) or mianserin (fig. 5B) treatment. Furthermore, $400 \mu \mathrm{g} / \mathrm{kg}$ of i.v. clonidine failed to reduce the dura-

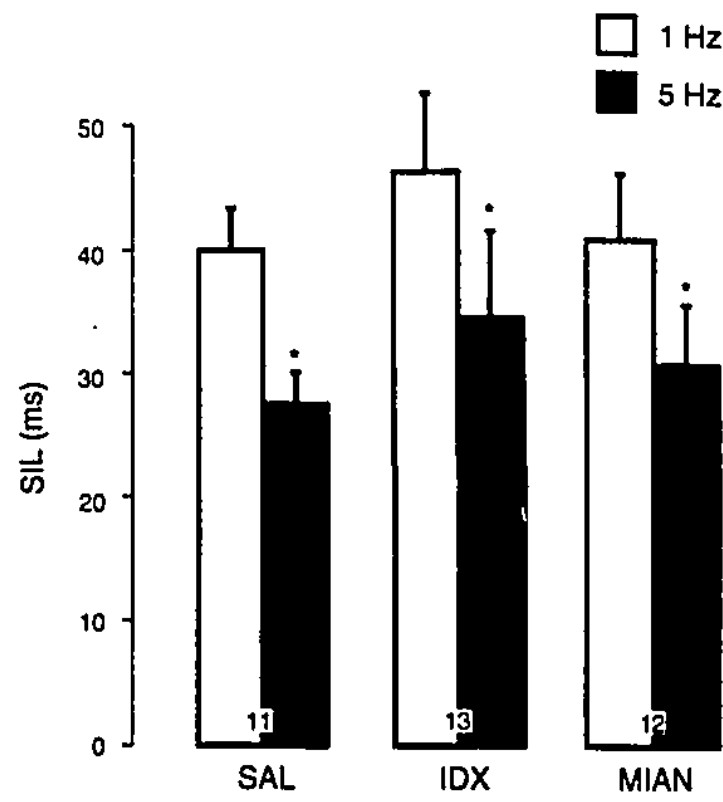

Fig. 2. Duration of suppression of firing activity of dorsel hippocampus pyramidal neurons obtained from electrical stimulations of the ascending 5-HT pathway at an intensity of $300 \mu \mathrm{A}$ and at trequencies of 1 or $5 \mathrm{~Hz}$. Data are means \pm S.E.M. In this and the following figures. The numbers within the histograms represent the number of neurons tested. The $5 \mathrm{~Hz}$ frequency of stmulation was significantly less effective than the $1 \mathrm{~Hz}$ frequency in suppressing the firing activity when tested on the same nourons ("P $<.05$, using the two-tailed paired Student's $P$ test). This degree of reduction was similar in rats treated with saline (SAL), idazoxan (IDX; $10 \mathrm{mg} / \mathrm{kg} /$ day) and mianserin (MIAN; $5 \mathrm{mg} / \mathrm{kg} /$ day) for 3 weoks. SIL, silence values.

A

B

SALINE

Prior to

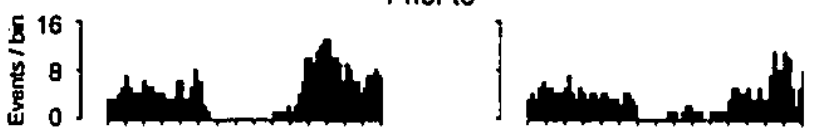

Following clonidine $10 \mu g / k g$

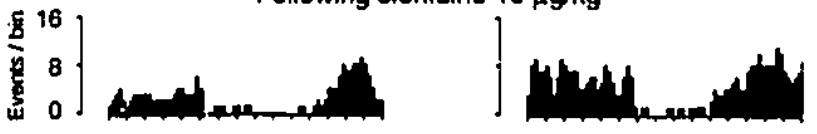

Following clonidine $400 \mu \mathrm{g} / \mathrm{kg}$
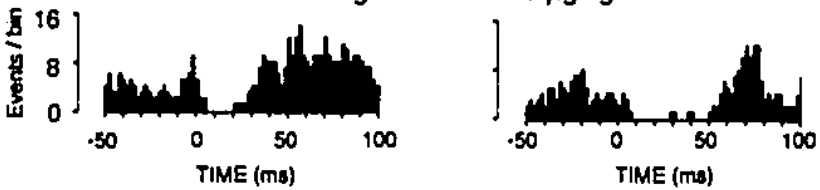

Fig. 3. Representative pertstimulus time histograms ilfustrating the effects of systernic injections of clonidine (10 and $400 \mu \mathrm{g} / \mathrm{kg} \mathrm{l.v.}$ ) on the efficacy of the electrical stmulation of the ascending 5-HT pathway in suppressing the firting activity of CA pyramidal neurons of hippocampus in a control rat (A) and in a rat treated with idazoxan (B). Each peristimulus time histogrem was constructed from 200 putses of $0.5 \mathrm{msec}$ delivered at $1 \mathrm{~Hz}$ at time 0 with an intensity of $300 \mu \mathrm{A}$. Bin width is $2 \mathrm{msec}$.

tion of suppression of firing below to the initial value after the idazoxan (fig. 5A) and the mianserin (fig. 5B).

Microiontophoretic applications of 5-HT and of NE were

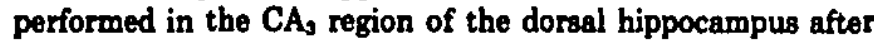
alpha-2 adrenergic antagonists treatments to assess their suppressant effects on the firing activity of the postaynaptic py- 


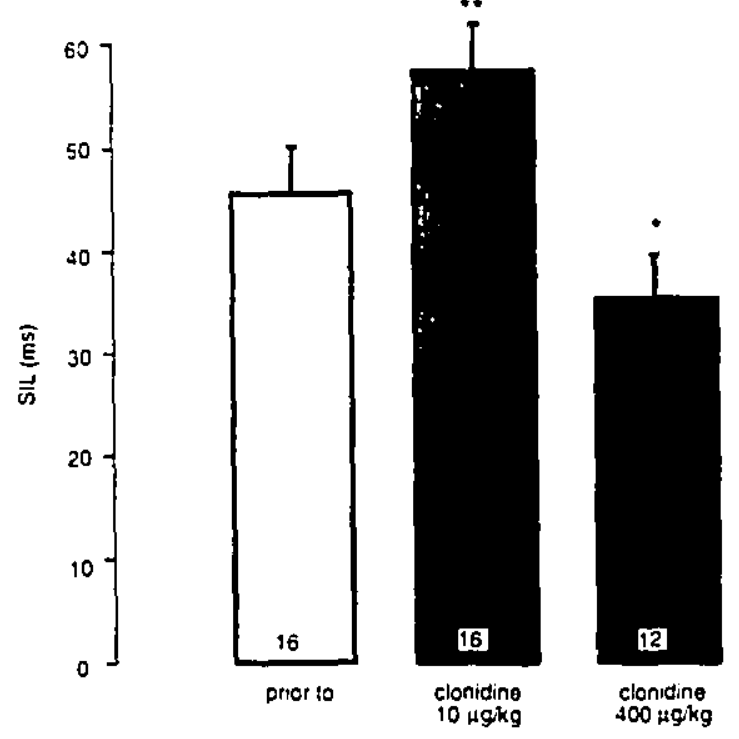

Fig. 4. Histograms showing the effects of systemic injections of cionidine on the efficacy of the electrical stimulation of the ascending 5-HT pathway in suppressing the firing activity of CA, pyramidal neurons of hippocampus in the control group. The number of neuron tested to assess the effect of the low and the high dose of clonidine is 16 and 12 , respectively. $" P<.01 ; " * p<.001$, by using a two-tailed paired Student's 1 test. comparing before and after clonidine administration in the same rats. SIL, silence values.

ramidal neurons. As shown in figure 6, no significant changes in the suppressant effects of 5-HT and NE were observed after long-term treatment with idazoxan or mianserin. Considering that the suppressant effect of $5 \cdot \mathrm{HT}$ is mediated by postsynaptic 5-HT 1 A receptors (Chaput and de Montigny, 1988) and that of NE through postsynaptic alpho-2 adrenergic receptors (Curet anc de Montigny, 1988; fig. 1), the results indicate an unchanged responsiveness of the alpha.2 adrenergic and $5 \cdot \mathrm{HT}_{1 \wedge}$ receptors of the pyramidal neurons of hippocampus.

Effects of repeated ECS. In another series of experiments rats received, over a 2-week period, six or seven ECS under light halothane anesthesia. During the same period, controls received halothane without ECS. Figure 7 shows representative illustrations of the suppressant effect of the electrical stimulation of the ascending 5-HT pathway on the firing rate of $\mathrm{CA}_{3}$ pyramidal neurons of the dorsal hippocampus in the ECS and the control group. Increases in the efficacy of the stimulation of $46 \%$ ( $P<.01)$ and $27 \%(P=.05)$ were found in the ECS group at 1 and $5 \mathrm{~Hz}$, respectively (fig. 8). However, the magnitude of the reduction in the duration of firing recorded when the frequency of stimulation was increased from 1 to $5 \mathrm{~Hz}$ in the ECS group $(-33 \pm 4 \%)$ was not different from that of the controls $(-34 \pm 7 \% ;$ fig. 8$)$, suggesting that the function of the terminal $5-H T_{10}$ autoreceptors had not been altered by the ECS treatment.

As can be seen in the histograms of figure 9 , the 10 and the $400 \mu \mathrm{g} / \mathrm{kg}$ i.v. doses of clonidine produced their usual incremental and decremental effects in the ECS group. The small dose produced in this group an enhancement of $19 \pm 6 \%$, which was smaller from that found in the control group ( $33 \pm 8 \%$; fig. 4), but the difference between these two groups was not statistically significant using covariance analysis. The decrease in the effectiveness of the stimulation of the 5-HT pathway by the high dose of clonidine in ECS-treated rats $(-29 \pm 10 \%)$

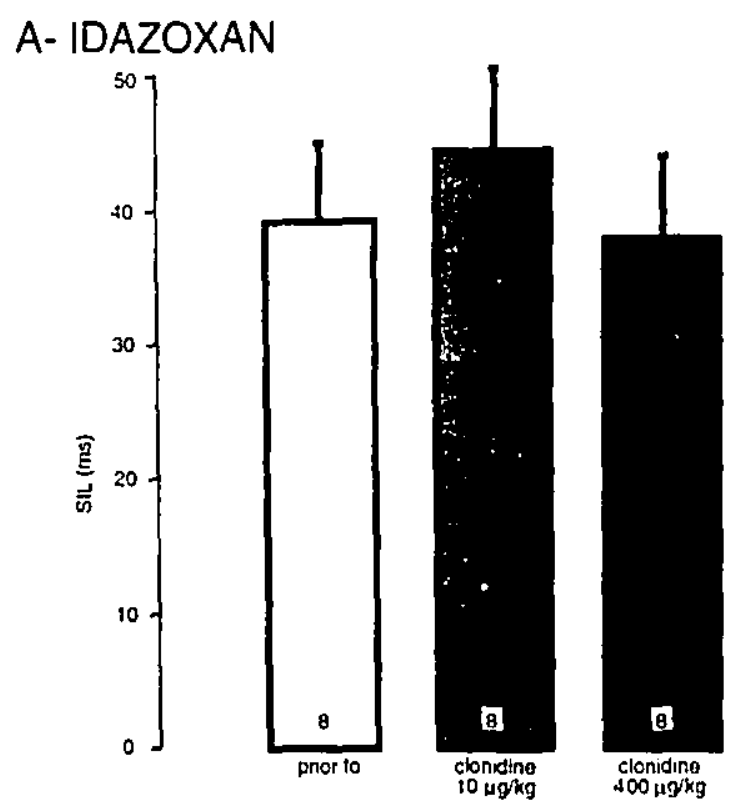

\section{B- MIANSERIN}

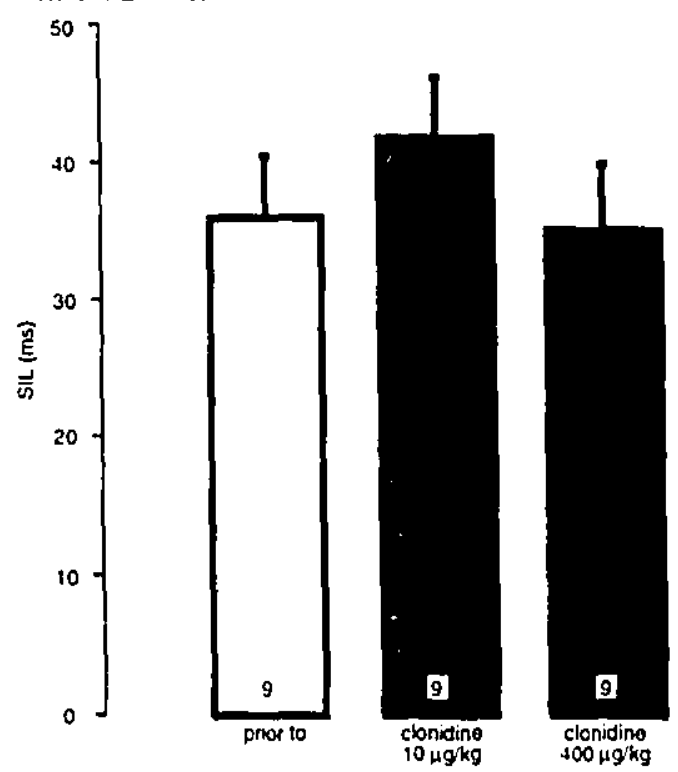

Fig. 5. Histograms showing the effect of systemic injections of clonidine on the efficacy of the electrical stimulation of the ascending 5-HT pathway in suppressing the firing activity of CA, pyramidal neurons of hippocampus in the ldazoxan (A) and the mianserin (B) treated group. The number of neurons tested is given at the bottom of each column. None of the mean silence values (SIL) after clonldine administration in the idazoxanand mianserin-treated groups were significantly different from those before clonidine administration in the same rats $(P>.05$ using paired two-tailed Student's $t$ test).

was similar to that found in the control group $(-24 \pm 5 \%$; fig. 4).

Changes in postsynaptic receptor responsiveness were found to parallel those observed in the efficacy of the stimulation of the 5-HT pathway. Indeed, as illustrated in figure 10 , the suppressant effect of microiontophoretically applied 5-HT, but not $\mathrm{NE}$, was increased after the ECS treatment. On average, there was a $148 \%$ enhancement of the suppressant effect of 5 HT in the ECS group compared to controls (fig. 11). In contrast, there was no significant change in the suppressant effect of $\mathrm{NE}$ (fig. 11). Thus, these results indicate an increase in the respon- 
5.HT

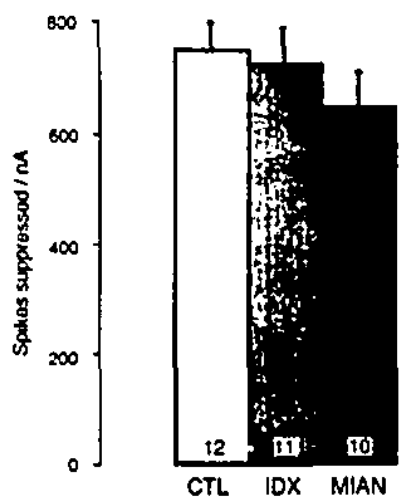

NE

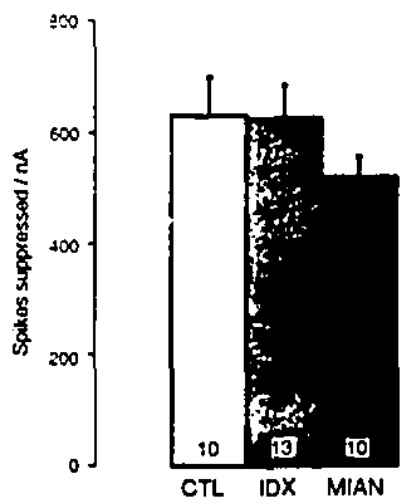

Fig. 6. Responsiveness, expressed in splkes suppressed per nanoanpere, of dorsal hippocampus pyramidal neurons to microlontophoretically applied 5-HT and NE in control (CTL), idazoxan (IDX) and mianserin (MIAN)-treated rats. The number of neurons tested is given at the hottom of each column. None of the mean values in the IDX-and MiAN-treated rats were significantly different from saline-treated rats $(P>.05$ by using two-tailed Student's ( test).

siveness of the 5-HT $1 \wedge$ receptors, but not alpha-2 adrenergic receptors, localized postsynaptically on $\mathrm{CA}_{3}$ pyramidal neurons.

\section{Discussion}

The present electrophysiological data show that a long-term treatment with an alpha-2 adrenergic antagonist decreases the inhibitory effect of a high dose of clonidine $(i n \mathrm{Hg} / \mathrm{kg}$ i.v.) on the effectiveness of the stimulation of the 5-HT pathway (figs. 4 and 5). In a previous study (Mongeau et al. 1993), the decremental effect of high doses of clonidine has been fully characterized by using the present electrophysiological paradigm. It was shown to be mediated by the activation of alpho. 2 adrenergic heteroreceptors on 5-HT terminals, resulting in a decreased amount of 5-HT released per action potintial. The present results thus suggest that mianserin and idezoxan desensitize alpha-2 adrenergic heteroreceptors. Such a change could result from a decrease in the density of the het: roreceptors, a change in the coupling of the heteroreceptors with their $G$ proteins or an alteration in second messenger function.

Idazoxan and mianserin most likely produced their lo 18 -term effects on these heteroreceptors indirectly, by increasinf during the treatment the concentration of NE through a blorkade of alpha-2 adrenergic autoreceptors of NE neurons. Although mianserin can block in vitro the reuptake of $\mathrm{NE}$, it does not produce this effect in vivo (Bauman and Maitre, 1977; Goodiet et al., 1977). A partial agonist effect of idazoxan at presynaptic alpha-2 adrenoceptors in rabbit ear artery has been reported (Limberger and Starke, 1983), but such an effect does not occur at the alpha-2 adrenergic heteroreceptors on 5-HT terminals, because idazoxan by itself does not decrease the efficacy of the stimulation of the 5-HT pathway when administered acutely (Mongeau et al., 1993).

There is evidence supporting our contention that idazoxan altered indirectly the responsiveness of alpha-2 adrenergic heteroreceptors on 5-HT terminals by acting preferentially on the alpha-2 adrenergic autoreceptors of NE neurons. Indeed, idazoxan, contrarily to the alpha-2 adrenergic antagonist yohim. bine, does not block the decremental effect of desipramine (a NE reuptake blocker) on the efficacy of the stimulation of the 5-HT pathway (Mongeau et al, 1993), whereas it readily blocks the decremental effect of desipramine on the efficacy of stimulation of the ascending NE pathway (Curet et al., 1992). This is in keeping with the 250 -fold greater affinity of idazoxan for autoreceptors than for the alpha-2 adrenergic heteroreceptors located on 5-HT terminals (Preziosi et al., 1989).

Mianserin also can increase NE release by the action of its (+)-stereoisomer which blocks the alpha-2 adrenergic autoreceptors on NE neurons (Raiteri et al., 1983b; Maura et al., 1985). It thus appears likely that the mianserin treatment produced its long-term effect on alpha-2 adrenergic heteroreceptors by a mechanism similar to that proposed above for the idazoxan treatment. Nevertheless, mianserin also blocks alpha2 adrenergic heteroreceptors (Raiteri et al., 1983b; Mongeau et al., 1993; Maura et al., 1985), which could oppose to the desensitizing action of the increased levels of endogenous NE elicited during the treatment by $(+)$-mianserin. For example, the desensization of beta adrenergic receptors by desipramine is impeded by the beta adrenergic antagonist propranolol (Wolfe et al., 1978). One might assume that the elevation in endogenous $\mathrm{NE}$ produced by the treatment was such that mianserin, at the dose used, did not prevent the desensitization of alpha-2 adrenergic heteroreceptors.

In addition, the results of the present study indicate that long-term treatments with alpha-2 adrenergic antagonists decrease the incremental effect of the small dose of clonidine (10 $\mu g / \mathrm{kg}$ i.v.; figs. 4 and 5). A previous electrophysiological study has shown that low doses of clonidine enhance the suppression of firing by preferentially activating alpha-2 adrenergic autoreceptors, thereby reducing the concentration of endogenous NE tonically activating alpha-2 adrenergic heteroreceptors on

$$
\text { A. CONTROL B. ECS }
$$
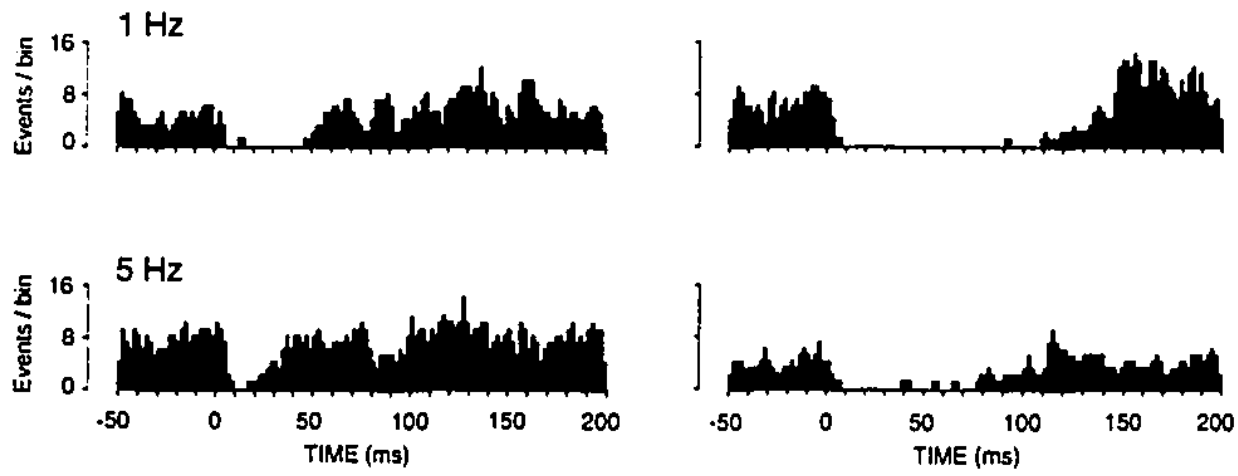

Fig. 7. Representative peristimulus time histograms illustrating the effect of the electrical stimulation of the 5-HT pathway in suppressing the firing activity of CA, pyramidal neurons of hippocampus at 1 or $5 \mathrm{~Hz}$ in a control (A) and an ECS-treated (B) rat. Each peristimulus time histogram was constructed from 200 pulses of 0.5 msec deliv. ered at time 0 with an intensity of $300 \mu \mathrm{A}$. Bin width is 2 msec. 


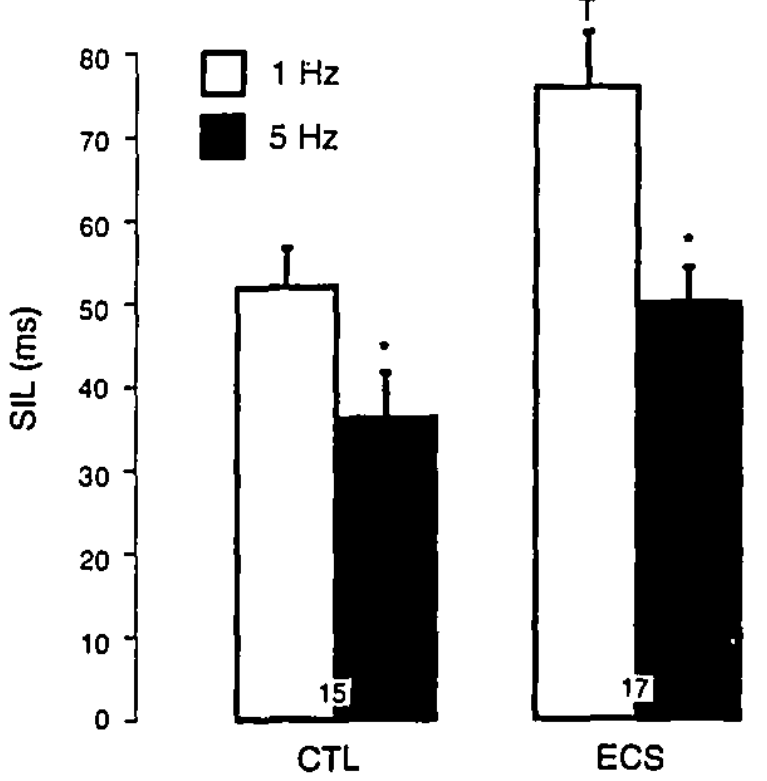

Fig. 8. Duration of suppression of firing activity of dorsal hippocampus pyramidal neurons obtained from electrical stimulations of the ascending 5.HT pathway at an intensity of $300 \mu \mathrm{A}$ and at frequencies of 1 and 5 Hz. The numbers within the histograms represent the number of neurons tested. The efficacy of the stimulation at $1 \mathrm{~Hz}$ was increased significantly in the ECS compared to the control (CTL) group (TP $<.01$, by using the unpaired two-tailed Student's ( test). The $5 \mathrm{~Hz}$ frequency of stimulation was skgnificantly less effective than the $1 \mathrm{~Hz}$ frequency in suppressing the firing activity when tested on the same neurons ("P $<.05$, by using the two-tailed paired Student's $t$ test). The degree of reduction was similar in the CTL and the ECS group. SIL, silence values.

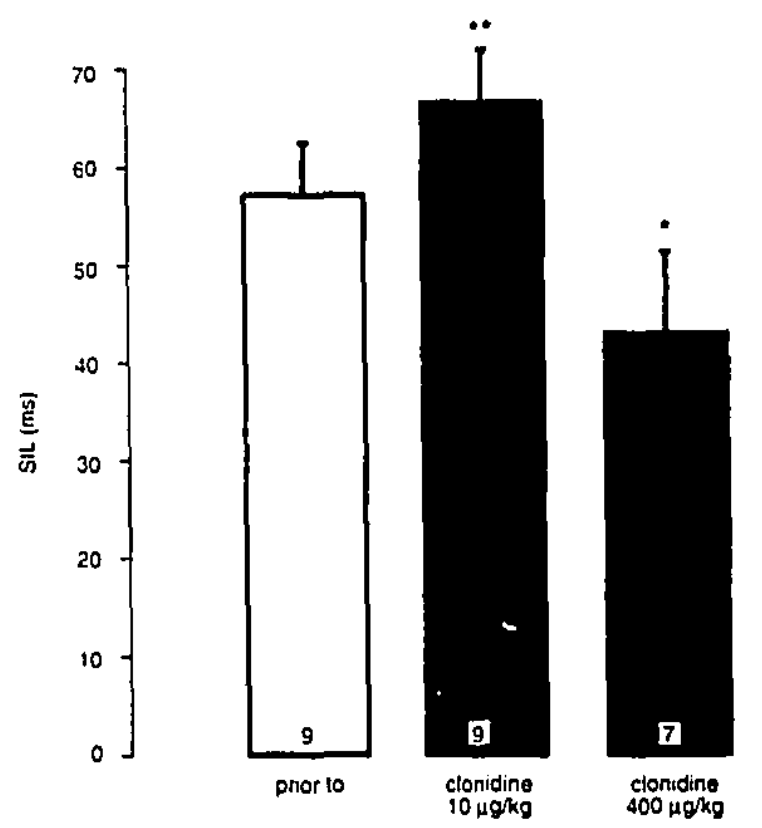

Fig. 9. Histograms showing the effect of systemic injections of chonidine on the efficacy of the electrical stimulation of the ascending 5-HT pathway in suppressing the firing activity of CA, pyramidal neurons of hlppocampus in the ECS group. The number of neurons tested to assess the effect of the small and the high dose of clonidine is 9 and 7 , respectively. - $p<.05$; $" p<.01$, by using a twa-tailed paired Student's $t$ test, comparing before and after clonidlne administration in the same rats. SIL, sllence values.
A. CONTROL
B. ECS

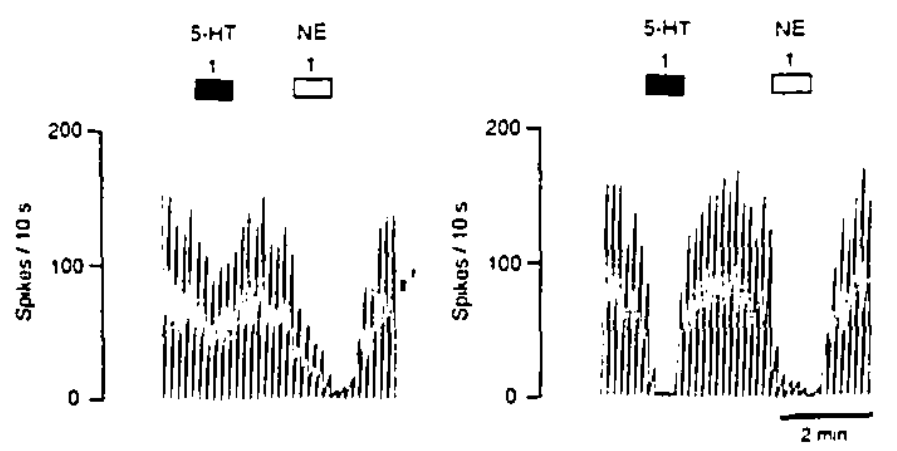

Fig. 10. Integrated firing rate histograms of two CA, dorsal hippocempus pyramidal neurons showing their responses to microiontophoretically applied 5-HT and NE in a control rat (A) and in a rat treated with repeated ECS (B). The bars indicate the duration of the application $(50 \mathrm{sec})$ for which the ejection current is given in nanoamperes. Time base applies to both traces.

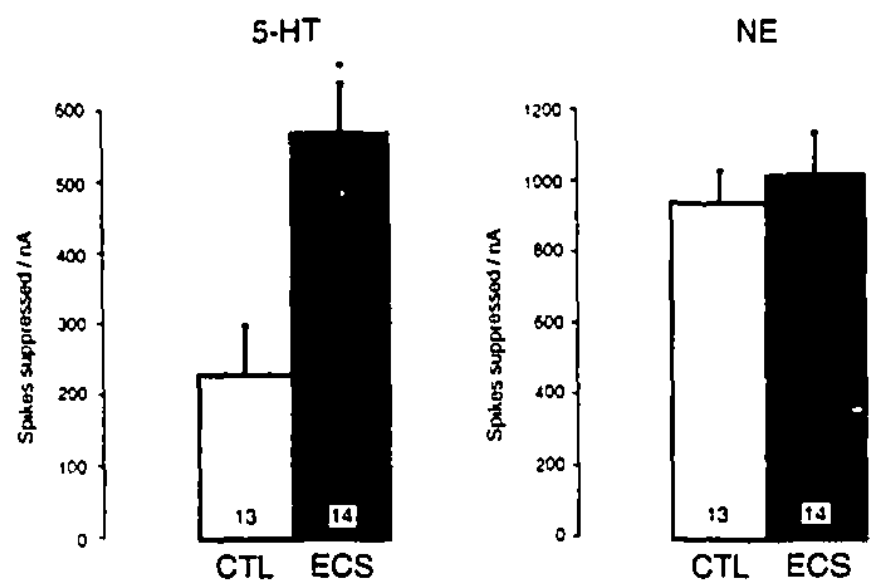

Fig. 11. Responsiveness, expressed in spikes suppressed per nanoampere, of dorsal hippocampus pyramidal neurons to microiontophoretically applied 5.HT and NE in control (CTL) and repeated ECS-treated rats. The number of neurons tested is given at the bottom of each column. The suppressant effect of 5-HT was increased significantly in the ECS group compared to the CTL group ("P $<.01$, by using the unpaired two tailed Student's t test), whereas that of NE was not.

5-HT terminals (Mongeau et al., 1993). The present data thus suggest a decrease tonic inhibitory action of endogenous NE on these heteroreceptors after idazoxan and mianserin treatments, $48 \mathrm{~h}$ after the minipumps were removed. This would be consistent with previous reports showing decreased levels of $\mathrm{NE}$ in the rat brain after a 10 -day treatment with idazoxan delivered through osmotic minipump (Dickinson et ah, 1990) and a supersensitivity of alpha-2 adrenergic autoreceptors after their chronic blockade with mianserin and idazoxan (Sugrue, 1980; Cerrito and Raiteri, 1981; Raiteri et ah, 1983a; Dickinson et al, 1989). An increased negative feedback regulation of $\mathrm{NE}$ release through the autoreceptors would obviously reduce the amount of endogenous NE acting on the alpha-2 adrenergic heteroreceptors at the moment of the experiment. The tonic inhibitory action of endogenous NE on 5-HT neurotransmission would thereby be so weak that a further activation of the alpha-2 adrenergic autoreceptors with the small dose of clonidine would have little or no detectable effect.

Raiteri et al. (1983a) reported no change in the sensitivity of the alpha-2 adrenergic heteroreceptors from 5-HT nerve end- 
ings of the rat brain after long-term treatment with mianserin. Two factors can readily be excluded to explain the discrepancy of this study with the present data. First, in the siudy of these investigators (Raiteri et al, 1983a), the treatments were performed with either the $(+)$ - or the $(-)$-stereoisomers of mianserin, but not with racemic ( \pm )-mianserin. The desensitization of the heteroreceptors might occur only with racemic $( \pm)$ mianserin, which is the compound used clinically. However, we do not believe this explanation likely, inasmuch as only the $(+)$-stereoisomer is active at the alpho-2 adrenergic autoreceptors and thus can contribute to desensitize alpha-2 adrenergic heterorsceptors by increasing NE release. Second, as in the study of Raiteri et al. (1983a), the washout period ( 2 days) was appropriate considering that there was no change in the responsiveness of postsynaptic alpha-? adrenoceptors to microiontophoretically applied NE after mianserin or idazozan treatment in the present study (fig. 6). One can thus assume that no residual drug could have blocked the effect of clonidine on alpha-2 adrenergic heteroreceptors. On the other hand, the difference that might be crucial is that Raiteri et al (1983a) injected the drug i.p. once daily for 14 days, whereas in the present study mianserin was delivered continuously during 21 days with osmotic minipumps inserted s.c. Intraperitoneal administration of mianserin in rats might not produce sustained alpha-2 adrenergic autoreceptor blockade, considering the important first-pass hepatic metabolism and also the much faster catabolism of such drugs in rats than in humans.

Contrarily to the idazoxan and mianserin treatments, repeated ECS increased the efficacy of 5-HT synaptic neurotransmission, as determined by the increased duration of suppression of furing of pyramidal neurons by the electrical stimulation of the afferent 5-HT pathway (fig. 8). This is consistent with a previous report (Chaput et ah, 1991), and can be correlated with an increased responsiveness of postsynaptic 5-HT 1 receptors to microiontophoretically applied 5-HT (fig. 11) (de Montigny, 1984; Chaput et ah, 1991). On the other hand, the increuse in 5-HT neurotransmission induced by repeated ECS does not appear to be related to an action of NE on alpha-2 adrenergic heteroreceptors. Indeed, there were no significant differences in the incremental and decremental effects of clonidine on the efficacy of the stimulation of the 5.HT pathway in ECS-treated and control rats (fige. 3 and 8 ). The effect of acute and repeated ECS on the release of endogenous NE was investigated recently in the rat (Thomas et al, 1992); in the hippocampus, acute ECS produced a transient $30-\mathrm{min}$ increase of NE levels, whereas repeated ECS did not change basal NE levels 24 h after the last treatment. In the light of these data, it can be presumed that alpho-2 adrenergic heteroreceptors on 5-HT terminals in the hippocampus are not exposed to a sustained increase in NE synaptic concentration during the course of a ECS treatment, explaining the lack of effect of this treatment on the responsiveness of the heteroreceptors to clonidine (figs. 3 and 8). It would be interesting to study the effect of ECS in the frontal corter, because a substantial increase in the levels of NE was observed in this region $24 \mathrm{hr}$ after repeated ECS (Thomas et al. 1992).

In conclusion, various classes of antidepressant treatments (MAO inhibitor, NE reuptake inhibitor and alpha-2 adrenergic antagonist), but not all (5.HT reuptake blocker, repeated ECS), share the capacity to desensitize the alpha-2 heteroreceptors on 5-HT terminals in the hippocampus by producing a sustained enhancement in the synaptic concentration of NE. This desensitization process might contribute to their therapeutic efficacies.

\section{Roferences}

BAUman, P. A. AND Mutrex, L.: Blockede of preayneptic alphe receptors and of amine uptake in the rat brain by the entidepreasent minnerino. NaunynSchmiedebert's Arch. Pharmacol. 300: 31-37, 1977.

BLRR, P. AND DE MONTIGNY, C.: Electrophytiolorical investigations on the effect of repested zimelidine adminintration on eorotonergic neurotransmission in the rat. J. Neurouci. 3: 1270-1278, 1983.

BLIER, P. AND DE MONTIGNY, C.: Short-term lithium administration enhancen serotonerric neurotranemistion: Electrophysiolorical evidence in the rat CNS. Eur. J. Pharmacol. 113: 69-77, 1985.

CERRTTO, F. AND RATrzR1, M.: Supersensitivity of central noradrenergic presynaptic sutoroceptors following chronic treatment with the antidepreasant mianserin. Eur. J. Pharmecol. 70: 425-126, 1981.

ChapUt, Y., Blier, P. AND de Montigny, C.: In vivo electrophysiological evidence for the refulatory role of autoreceptort on serotonergic cerminals. J. Neuracici. 6: 2796-2801, 1986.

ChapUT, Y. AND dE MONTIGNY, C.: Effects of the 5-hydroxytryptamine receptor antaconist, BMY 7378, on 5-hydroxytryptamine neurotransmistion: Electro. phytiological itudiea in the rat central nervous eyatem. J. Pharmacol. Exp. Ther. 246: 359-370, 1988.

ChapUt, Y., de MontrGery, C. AND BLtan, P.: Effecte of a celective 5.HT reuptake blocker, citalopram, on the sensitivity of S.HT sutoreceptorn: Elec. trophyaiological atudies in the rat brain. Neunyn-Schmiedebert's Anch. Pharmacol. 333: 342-348, 1986b.

Chaput, Y., de Montigny, C. and Bluer, P.: Preaynaptic and postoynaptic modifications of the ecrotonin system by long-Lerm administration of antide. prosennt trestmente: An in vivo electrophyaiologic atudy in the rat. Neuropey. chopharmacolory 8: 219-229, 1991.

CURST, O. AND DE MONTIONY, C.: Eloctrophyaiolonical characterization of adrenoceptors in the rat doral hippocampus. I. Recepton mediating the effect of microiontophoretically applied norepinephrine. Brain Res. 478: 35-46, 1988.

CURE, O. AND DE MONTIGNY, C.: Eloctrophysiological charecterization of adrenocepton in the rat doral hippocampun. III. Evidence for the phytiolopical role of terminal aredrenerric autorecepton. Brain Res. 489: 18-26, 1989.

CURET, O., DE MONTIGNY, C. AND BLIER. P.: Effect of detipramine and amphetamine on noradrenergic neurotrnamisaion: Electrophyaiological studies in the rat brain. Eur. J. Phnrmecol. 221: 59-70, 1992.

DE MONTIONY, C.: Eloctroconvulaive ohock treatments enhance retpontiveness of forebrain neturone to merotonin. J. Pharmecol. Exp. Ther. 228: 230-234, 1984.

Dennig, T. L'Heureux, R., Carter, C. and Scatton, B.: Preaynaptic $\alpha_{1}$ edrenoceptors play a major rolo in the effocts of idezoran on cortical noradrenaline rolean (o mesarred by in vivo dialyis) in the rat. J. Pharmacol. Exp. Ther. 241: 642-649, 1987.

Dickinson, S. L., Gadie, B., Havlez, M. E., Hunter, C. and Tulloch, I. F.: Behaviourd effocts of idszoxan given continuounly by otmotic minipump in the rat. Br. J. Pharmacol. 88: 932p, 1989.

Dickinson, S. L., Gadie, B., Haynes, M. J., Lane, A. C., Walter, D. S. AND WalthaM, K.: Neurochemical effects of idezoren given continuously by osmotic minipump in the rat. Br. J. Pharmacol. 90: 274p, 1990.

DoXeY, J. C., Ronch, A. G. AND SMrTH, C. F. C.: Studies on RX781094: A solective, potent and epocific antagonist of $\alpha_{\mathrm{r}}$-edrenoceptors. Br. J. Pharmecol. 78: 489-505, 1883.

FrunxhULzzeN, A. L. AND MULDER, A. H.: Noradrenaline inhibits depolarization-induced 'H-5-HT relenee from olices of rat hippocempus. Eur. J. Pharmancol. 63: 179-182, 1980

FrunkHULXRN, A. L. AND MULDER, A. H.: Pharmecological characterization of preaynaptic alphe-edrenoceptor trom modulating ' $\mathrm{H}$ - NA and ${ }^{~} \mathrm{H} \cdot 5 \cdot \mathrm{HT}$ release form alices of the hippocampus of the rat. Eur. J. Pharmecol. 81: 97-106, 1982.

Goodtet, I., MIRzylees, S. E. AND SUGRUE, M. F.: Effect of mianuerin, a new antidepreanent, on the in vitro and in vivo uptake of monosmines. Br. J. Pharmecol. 61: 307-313, 1977.

GOTHERT, M. AND HUTH, H.: Alpha-adrenoceptor-medinted modulation of 5-HT releaw from rat brein cortox alices. Naunyn-Schmiedeterg'o Arch. Pharmacol. 313: 21-26, 1880 .

GOTHERT, M., HUTH, H. AND SCHLICKER, E.: Churacterization of the receptor subtype involvod in atpha-edrenocoptor-mediatod modulation of cerotonin relente from rat brain cortex alices. Naunyn-Schmiedoberg's Arch. Pharmacol. S17: 199-203, 1981.

KANDEL, E. R. AND SPENCER, W. A.: Electrophyaiolory of hippocampal neuron. II. Aferpotantials and repetitive firine. J. Neurophyaiol. 24: 243-259, 1961.

L'Heuretx, R., Dennis, T., Curst, O. AND Scatton, B.: Menurement of ondogenow noradrenaline releane in the rat cerebral cortex in vivo by trans. cortical dialyais: Effects of druge affecting noradrenertic tranemiation. $J$. Nourochem. 16: 1794-1801, 1986.

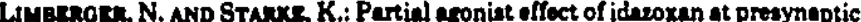
ar-edronoceptors in rabbit ear artory. Neunyn-Schmiodeberf's Arch. Phermacol. 324: 75-78, 1983

MaURu, G., Grutionani, A. AND Rurtza, M.: Noredrenaline inhibits central cerotonin rolean throuth ar-edrenocepton located on merotonertic nerve terminala. Neunyn-Schmiodibort's Arch. Pharmecol. 320: 272-274, 1982. 
Maura, G., Gemicnanj, A. AND Rartert, M.: a. Adrenoceptors in rat bypothalamu and cerebral cortex: Functional evidence for pharmacologically distinct subpopulations. Eur. J. Pharmacol. 116: 335-339, 1985.

MONGeAU, R. BLIER, P. AND DE MONTIGNY, C.: In vivo electrophysiological evidence for tonic activation by endorenous noradrenaline of $\alpha_{\mathfrak{g}}$-adrenoceptors on 5-hydroxytryptumine terminals in the rat hippocampus. Naunyn-Schmiedebers'o Arch. Pharmacol. 347: 266-272, 1993.

Mongeav, R. DE MONTIGNY, C. AND ButR, P.: Electrophysiological evidence for the desensitization of $a_{3}$-adrenocepton on serotonin terminals following long-term treatment with drug increasing norepinephrine synaptic concentra. tion. Neuropaychopharmacolopy, 10: 41-51, 1994

Osman, O. T., RudorteR, M. V. AND POTtER, W. Z.: Chronic $\alpha_{\text {, antagonism }}$ incresses norepinephrine, blocks GH release and is antidepreasent. Biol. Psy. chintry 26: 197A, 1989.

PINDER, R. M. AND SITSEN, J. M.: a,-Adrenoceptor antaronisto as antidepressants: The search for selectivity. Puychophermecol. Series 3: 107-112, 1987.

Preztogi, P., Martikz, M., Navarra, P., Pistritto, G. and Vacca, M. Prolectin-lowering abjlity of $( \pm)$-jderoran may be linked to a central noradrenergic-nerotonerric interplay. J. Pharmacol. Exp. Ther. 240: 256-263, 1989.

RuTrza, M., Marchi, M. AND MaURa, G.: Chronic drut treatmente induce changes in the censitivity of preaynaptic autoreceptor but not of preaynaptic heteroreceptor. Eur. J. Phermecol. 81: 141-143, 1983 .

Ramert. M., Mauza, G., Gemignant, A. and Prttaluga, A.: Differential blocked by (-)minnerin of the $\alpha_{\mathrm{r}}$-adrenocepton mediating inhibition of noradrenaline and serotonis releace from rat brain aynaptosomea. Naunyn. Schmiedaber's Arch. Phermecol. 322: 180-182, $1983 \mathrm{~b}$.

RANCK, J. B.: Behavioral correlatee and firing repertoires of neurons in the doral hippocampal formation and septum of unreatrained rate. In The hippocampus. ed. by I. Iaracson and R. Lee, pp. 207. PJenum Pres, New York, 1975

Rose, A. MCCulloch, M. W., SArantos-Laska, C. AND Rand, M. J.: Effecte of miancerin on noradrenergic mechnnisms. J. Paychintr. Ret. 18: 79-88, 1984 STARKE, K. AND MONTEL, H.: Involvement of alpha-receptor in clonidine induced inhibition of tranamitter relesse from central monoemine neurones. Neuropharmacolozy 12: 1073-108U, 1973.

SUGRUE, M. F.: The inability of chronic mianserin to blocic central az-adrenoceptors. Eur. J. Pharmacol. 68: 377-380, 1980.

TAYLOR, M. A.: Idazoran: A selective $a_{2}$-antagonist and effective auseninod antidepresant in two bipolar depreaned patiente. Arch. Gen. Psychintry 46: 958-959, 1989.

Thomas, D. N., NUTT, D. J. AND HOLMAN. R. B.: Effecte of acute and chronic electroconvilaive shock on noradrenaline release in the rat hippocampun and frontal cortex. Br. J. P harmacol. 108: 430-434, 1992.

van Riezen, H., Pinder, R. M. Nickolson, V. J., Hobbelen, P., Zared, I. AND VAN ORR VeEN, F.: Miansorin, a really different antideprasant. in Pharmacological and Biochemical Properties of Drus Substances, vol. 3, ed by M. E. Goldbert, pp, 1-38, American Pharmeceutical Associntion and Academy of Pharmeceutical Sciences, Wahinton, 1981.

Wolne, D. B., Harden, T. K., SPORN, J. R. AND MoLjnotr, P. B.: Presynaptic modulation of bete adrenerpic roceptors in rat cerebral cortex aftar treatment with antidepresoanta. J. Pharmecol. Exp. Ther. 207: 305-312, 1978.

Sond reprtat requenta to: Dr. Raywond Mongear:, Depurtanent of Paychintry, Neurobiolopical Poychintry Unit, McGill Univernity, 1033 Pine Ave. Went, Montréal, Quebec, Canada H3A $1 \mathrm{Al}$. 


\section{Fourth article}

The second step of this Ph.D. thesis was to investigate the opposite NA - 5-HT interaction, that is, 5-HT receptors of the rat hippocampus modulating the release of NA. We have decided to focus on the possible involvement of 5-HT3 receptors, having already had some indications from the literature that the 5-HT3 agonist 2-methyl-5-HT modulates the release of $\mathrm{NA}$ in other species and brain areas. The technique of in vitro release of tritiated neurotransmitter from brain slices was used in the present study. In brief, slices of the rat hippocampus, hypothalamus or frontal cortex were preloaded with [3H]NA and then placed into glass chambers where they were superfused with a physiological medium. The slices were electrically stimulated twice (first at $S_{1}$ and then at $\mathrm{S}_{2}$ ) and the effect on the evoked release of [3H]NA of various 5-HT agents, introduced before $\mathrm{S}_{2}$, was estimated by calculating the $\mathrm{S}_{2} / \mathrm{S}_{1}$ ratios.

This article entitled "Activation of $5-H T 3$ receptors enhances the electrically evoked release of $[3 \mathrm{H}]$ ]noradrenaline in rat brain limbic structures" by myself, Claude de Montigny and Pierre Blier was published in the European Journal of Pharmacology (1994, vol. 256, pp. 269-279). 


\title{
Activation of 5- $\mathrm{HT}_{3}$ receptors enhances the electrically evoked release of $\left[{ }^{3} \mathrm{H}\right]$ noradrenaline in rat brain limbic structures
}

\author{
Raymond Mongeau *, Claude De Montigny, Pierre Blier

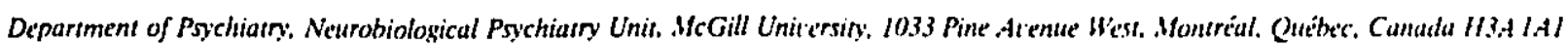

(Received 29 September 1993: revised MS received 27 December 1993: accepted \& Februany 1994)

\begin{abstract}
The ability of 5-HT receptor agonists to modulate the electricully evoked release of $\left[{ }^{3} \mathrm{H}\right.$ ]noradrenaline was tested on preloaded slices of the rat brain. The 5-HT, receptor agonist 2-methyl-5.hydroxytryptamine (2-methyl-5-HT) (16-100 $\mu \mathrm{M})$ concentration-dependently enhanced the electrically evoked release of [ ${ }^{3} \mathrm{H}$ ]neradrenaline in the hippocampus and the hypothalamus, but only at $100 \mu \mathrm{M}$ in the frontal cortex. The enhancing effect of $2 \cdot$ methyl-5-HT was blocked by the $5 \cdot \mathrm{HT}$, receptor antagonist ondansetron. Elevated levels of endogenous 5-HT, achieved through selective reuptake blockade with paroxetine, as well as the addition of exogenous 5.HT in the medium. also enhanced $\left[{ }^{3} \mathrm{H}\right]$ noradrenaline release. Furthermore. this effect of paroxetine was blocked by nanomolar concentrations of the 5.HT, receptor antagonists ondansetron. tropisetron and $(S)$. zacopride. Only high concentrations of the $5 \cdot \mathrm{HT}_{3}$ receptor agonist $m$-chlorophenylbiguanide increased $\left[{ }^{3} \mathrm{H}\right]$ noradrenaline release from hippocampal slices, and this effect was not blocked by ondansetron nor by $(S)$-zacopride. The possibility that the enhancing effect of 2-methyl-5-HT could have been due to the antagonism of $\alpha_{2}$-3utoreceptors of noradrenergic terminals was ruied out by the unaltered effectiveness of the $\alpha_{2}$-adrenoceptor agonist UK-14.304 $(1 \mu \mathrm{M})$ to attenuate [ $\left.{ }^{3} \mathrm{H}\right]$ noradrenaline release in the presence of $100 \mu \mathrm{M}$ of 2-methyl-5-HT. Moreover, in pseudo-one-pulse experiments $100 \mu \mathrm{M}$ of 2-methyl-5-HT increased [ ${ }^{3} \mathrm{H}$ )noradrenaline release in the absence of autoinhibition through $\alpha_{2}$-adrenergic autoreceptors. The 5-HT ${ }_{1 \mathrm{~A}}$ and 5-HT ${ }_{13}$ receptor agonists 8-hydroxy-2(di-n-propyl-aminotetralin and CP-93,129, respectively, as well as the 5-HT receptor $^{2}$ agonist 5-carboxyamidotryptamine, were devoid of effect on the release of ['H]noradrenaline. The $5-H T_{2 A} / 2 \mathrm{C}$ receptor agonist 1-(2.5-dimethoxy-4-iodopheny))-2-aminopropane increased the release of $\left[{ }^{3} \mathrm{H}\right]$ noradrenaline. but this cffect was not blocked with the 5.- $\mathrm{HT}_{3}$ receptor antagonist ondansetron. Lesioning 5- $\mathrm{HT}$ fibers with the neurotoxin 5.7-dihydroxytryptamine did not alter the action of 2-methyl-5-HT on [ $\left.{ }^{3} \mathrm{H}\right]$ noradrenaline release. indicuting that this effect is not attributable to an action of this $5-\mathrm{HT}_{3}$ receptor agonist on 5 -HT terminals.
\end{abstract}

Key words: 5-Carboxyamidotryptamine; CP-93.129; Desensitization; 5.7-Dihydroxytryptamine: Hippocampus: Hypothalamus: Frontal cortex; Tropisetron; 5-HT (5-hydroxytryptamine, scrotonin): 8-OH-DPAT (8-hydroxy-2(di-n-propylamino)tetralin); 2Methyl-5.HT: $m$-Chlorophenylbiguanide; Ondansetron: Paroxetine: Presynaptic modulation: $(S)$-Zacopride

\section{Introduction}

Numerous studies have shown the ability of 5-HT (5-hydroxytryptamine, serotonin) to modulate noradrenaline and dopamine neurotransmissions. In the peripheral nervous system, activation of $5-\mathrm{HT}_{3}$ receptors depolarizes sympathetic postganglionic neurons and consequently triggers noradrenaline release in rabbit and rat superior cervical ganglia (Fozard, 1984), as well as in the pig mesenteric ganglion (Meehan and

\footnotetext{
Corresponding author. Tel. (514) 398-7304, fax (514) 398-4866.
}

Kreulen, 1991). On the other hand, the release of noradrenaline from sympathetic nerves in certain human and rat blood vessels is inhibited through presynaptic 5-HT receptors (Göthert et al.. 1986; Molderings et al., 1987, 1990), and in the pig vessels through presynaptic 5-HT receptors different from the $5-\mathrm{HT}_{1}$. $5-\mathrm{HT}_{2}$ or 5- $\mathrm{HT}_{3}$ receptor class (Molderings et al., 1989; Mechan and Kreulen, 1991). In the central nervous system, the dorsal and the median raphe exert an inhibitory action on the firing activity of the locus coeruleus neurons (Lechin et al., 1989). Synaptic potentials evoked by glutamate and $\gamma$-aminobutyric acid (GABA) in the rat locus coeruleus are inhibited 
through $5-\mathrm{HT}_{1,}$ and $5-\mathrm{HT}_{13}$ receptors (Bobker and Williams, 1989). On the other hand, the activation of 5-HT, receptors facilitates the sensory-evoked firing activity and decreases the spontaneous activity of noradrenergic neurons in this nucleus by GABA and excitatory amino acid inputs. respectively (Rasmussen et al., 1986; Aghajanian, 1980: Chiang and Aston-Jones, 1993). Numerous reports have also shown that dopaminergic neurotransmission is enhanced by the activation of $5-\mathrm{HT}_{3}$ receptors (Blandina et al., 1988 1989; Jiang et al., 1990; Chen et al., 1991, 1992). In contrast, few investigations have focussed on the effect of 5-HT agents on the function of noradrenergic terminals. A recent study has reported that the potassiumevoked release of endogenous noradrenaline in rat hypothalamic slices is decreased by $5-\mathrm{HT}_{3}$ receptor activation (Blandina et al., 1991). However, this is at variance with other data showing a facilitatory effect of 5-HT on noradrenergic terminals in different preparations. The 5-HT receptor agonist 5-methoxy- $N, N$-dimethyltryptamine and the electrical stimulation of the descending 5-HT pathway of rats pretreated with a 5-HT reuptake blocker increase the efflux of endogenous noradrenaline in the rat spinal cord (Hammond et al., 1985: Reimann and Schneider, 1993). In addition, a study by Feuerstein and Hertting (1986) carried out in rabbit hippocampus showed that exogenous 5-HT, 5-HT reuptake blockade, and the $5-\mathrm{HT}_{3}$ receptor agonist 2-methyl-5-HT increase the electrically induced release of $\left[{ }^{3} \mathrm{H}\right]$ noradrenaline. These effects were blocked by the 5-HT, receptor antagonists MDL-72222 and ICS 205-930 (tropisetron). However, 5-carboxyamidotryptamine $(5-\mathrm{CT})$, a $5-\mathrm{HT}$, receptor agonist without significant affinity for $5-\mathrm{HT}_{3}$ binding sites (Hoyer 1991), produced a similar enhancement of the croked release of $\left[{ }^{3} \mathrm{H}\right]$ noradrenaline.

The present study on the effect of 5- $\mathrm{HT}_{3}$ ligands on $\left[{ }^{3} \mathrm{H}\right]$ noradrenaline release was undertaken to address several issues: (1) to test the potency of the agonist 2-methyl-5-HT in different limbic structures in order to determine whether its effect on the function of noradrenergic terminals varies among brain regions; (2) to assess whether the $5-\mathrm{HT}_{3}$ receptors modulating noradrenaline release desensitize rapidly after agonist exposure: (3) to assess the capacity of endogenous $5-\mathrm{HT}$ to alter $\left[{ }^{3} \mathrm{H}\right]$ noradrenaline release using a $5-\mathrm{HT}$ reuptake blocker and various $5-\mathrm{HT}_{3}$ receptor antagonists; (4) to rule out the possibility that 2-methyl-5-HT exerts its enhancing effect on $\left[{ }^{3} \mathrm{H}\right]$ noradrenaline release through a blockade of $\alpha_{2}$-autoreceptors of noradrenergic terminals.

It was also considered crucial to test the ability of various $5-\mathrm{HT}_{1}$ and $5-\mathrm{HT}_{2}$ receptor agonists to modulate the evoked release of $\left[{ }^{3} \mathrm{H}\right]$ noradrenaline and compare their actions to that of 5-HT. The results of Feuerstein and Hertting (1986) and Blandina et al.
(1991) suggested a participation of 5-HT, receptors in the modulation of noradrenaline release by $5-\mathrm{HT}$ and 2-methyl-5-HT in the brain. First, the 5-HT receptor receptor agonist 5-CT was found to enhance the electrically evoked release of $\left[{ }^{3} \mathrm{H}\right]$ noradrenaline in the rabbit hippocampus (Feuerstein and Hertting. 1986). Second. the blockade of $5-\mathrm{HT}_{2 \mathrm{~N} / \mathrm{CC}}$ receptors with ritanserin was required for the observation of the inhibition of the potassium-evoked release of endogenous noradrenaline induced by 5-HT, but not for that induced by 2 -methyl-5-HT. Furthermore. the activation of 5 - $\mathrm{HT}_{2 \mathrm{~A} / 2 \mathrm{C}}$ agonist receptors with $\alpha$-methyl-5-HT reduced the inhibitory effect of 2-methyl-5-HT (Blandina et al., 1991).

\section{Materials and methods}

Male Sprague-Dawley rats $(250-350 \mathrm{~g})$ were killed by decapitation and their brain immediately removed and rapidly dissected on an ice-cold glass plate. Slices $400 \mu \mathrm{m}$ thick and of around $3 \mathrm{~mm}^{2}$ of surface from the hippocampus, hypothalamus, or frontal cortex were prepared using a Mcllwain chopper and incubated for $30 \mathrm{~min}$ at $37^{\circ} \mathrm{C}$ in $\mathrm{Krebs}$ buffer containing $0.1 \mu \mathrm{M}$ $\left[{ }^{3} \mathrm{H}\right]$ noradrenaline (specific activity $15 \mathrm{mCi} / \mathrm{mmol}$ ) and bubbled with a mixture of $95 \% \mathrm{O}_{2} / 5 \% \mathrm{CO}_{2}$. The composition of the Krebs buffer in $\mathrm{mM}$ concentrations was: $\mathrm{NaCl} 118, \mathrm{KCl} 4.7, \mathrm{CaCl}_{2}$ 1.3. $\mathrm{MgCl}_{2}, 1.2$. $\mathrm{NaH}_{2} \mathrm{PO}_{4} 1, \mathrm{NaHCO}_{3}$ 25, glucose 11.1. $\mathrm{Na}_{2}$ EDTA 0.004 and ascorbic acid 0.11. At the end of the $\left[{ }^{3} \mathrm{H}\right]$ noradrenaline uptake period, the slices were washed in Krebs buffer to remove the free $\left[{ }^{3} \mathrm{H}\right]$ noradrenaline, and then one hypothalamus slice or ti:ree slices of the hippocampus or frontal cortex (five slices of the hippocampus were used in pseudo-onepulses experiments) were transferred into each gliss chamber (volume $1.85 \mathrm{ml}$ ) and superfused at rate of 0.5 $\mathrm{ml} / \mathrm{min}$ with oxygenated Krebs buffer maintained at $37^{\circ} \mathrm{C}$. Nineteen consecutive 4-min fractions were collected starting $60 \mathrm{~min}$ after the beginning of superfusion for hippocampus and frontal cortex slices and 52 min after for hypothalamus slices. The slices were stimulated twice, at $8 \mathrm{~min}\left(S_{1}\right)$ and at $56\left(S_{2}\right) \mathrm{min}$. after the end of the washing period. The electrical field generated in the chambers between the (wo platinum electrodes (2 $\mathrm{cm}$ apart) had a voltage drop of about 5 $\mathrm{V} / \mathrm{cm}$. The following stimulation parameters were normally used: $20 \mathrm{~mA}, 2 \mathrm{~ms}, 3 \mathrm{~Hz}$ for $2 \mathrm{~min}$. In pseudoone-pulses experiments the following parameters were used: $20 \mathrm{~mA}, 2 \mathrm{~ms}, 4$ pulses at $100 \mathrm{~Hz}$. The first stimulation period $\left(S_{1}\right)$ was always used as control and, when $5-\mathrm{HT}_{3}$ receptor antagonists were used to antagonize the action of agonists, they were present throughout the superfusion, whereas the other drugs were added 8 or $20 \mathrm{~min}$ before $S_{2}$ and remained present 
until the end of the experiment. Idazoxan and 2methyl-5-HT were added to the superfusion medium 20 min before $S_{1}$ to test their antagonism toward the inhibitory effect of UK-14.304 introduced $20 \mathrm{~min}$ before $S_{z}$. Nisoxetine, a selective noradrenaline reuptake inhibitor (Fuller et al.. 1979), was present threughout the superfusion period when necessary to avoid the displacement of $\left[{ }^{3} \mathrm{H}\right]$ noradrenaline from its storage vesicles by 5-HT receptor agonists. At the end of the experiments, the slices were solubilized in $0.5 \mathrm{ml}$ of Soluene 350 and the radioactivity in the slices and superfusate samples was determined by liquid scintillation spectrometry using $5 \mathrm{ml}$ of Ready Micro at $50 \%$ efficiency using a Beckman LS1600 counter. The amount of tritium released per 4-min sample was expressed as a fraction of the total tissue tritium content at the onset of the respective collection period. The overflow of tritium produced by the electrical stimulation was calculated as the total increase in radioactivity above the basal outflow of tritium determined in the sample immediately preceding the onset of stimulation. To assess the drug-induced changes of electrically evoked release of tritium from the slices preloaded with $\left[{ }^{3} \mathrm{H}\right]$ noradrenaline. $S_{2} / S_{1}$ ratios were calculated. and $B_{2} / B_{1}$ ratios were also calculated to determine whether the drugs had altered the basal outflow of radioactivity.

\subsection{Pretreatment with 5,7-dihydroxytnptamine}

A group of rat was treated 3 weeks before the experiments with a $0.9 \% \mathrm{NaCl}$ and $0.1 \%$ ascorbic acid solution containing $10 \mu \mathrm{g} / \mu$ l of 5.7-dihydroxytryptamine (5.7-DHT). $20 \mu$ l were injected intracerebroventricularly and $2 \mu 1$ were locally applied in both the dorsal and median raphe. To protect noradrenergic neurons from the neurotoxic action of 5.7-DHT, the selective noradrenaline reuptake blocker desipramine (25 mg/kg i.p.) was administered $1 \mathrm{~h}$ prior to the neurotoxin.

\subsection{Statistical analyses}

All results are expressed as means \pm S.E.M. Differences between the means of treated and control groups were tested with the two-tailed Studen's t-test, prerequisites of which were condidered using the Bartlett's test. Probability $(P)$ values smaller than 0.05 were considered as significant.

\subsection{Drugs}

The following drugs were used: 2-methyl-5-HT, 8hydroxy-2(di-n-propylamino)tetralin (8-OH-DPAT), 1 (2,5-dimethoxy-4-iodophenyl)-2-aminopropane $(( \pm)$. DOI), 5-CT (Research Biochemicals, Natick, MA.
USA), 5-HT creatinine sulfate, 5,7-DHT creatinine sulfate, idazoxan $\mathrm{HCl}$ (Sigma Chemicals, St. Louis, MO. USA), 3-[1.2.5,6-tetrahydropyrid-4-yl]pyrrolo[3.2b]pyrid-5-one (CP-93.129), 5-bromo-6-[2-imidazolin-2yl-aminol-quinoxaline (UK-14,304; Pfizer. Groton. CT. USA), [ ${ }^{3} \mathrm{H}$ ]noradrenaline $\mathrm{HCl}$ (NEN. Mississauga. Ont.. Canada), ondansetron (Glaxo. Montreal. Canada), tropisetron (ICS 205-930; Sandoz, Basel. Switzerland), (S)-zacopride (Delalande, Rueil-Malmaison. France). paroxetine (SmithKline Beecham. Harlow. UK), nisoxetine (Lilly, Indianapolis, IN, USA), $m$-chlorophenylbiguanide $\mathrm{HCl}$ (Cookson Chemicals, Nursling Southampton, UK).

\section{Results}

3.I. Effects of 5-HT, receptor agonists on the eloked release of tritiated noradrenaline

In the following experiments, the noradrenaline reuptake blocker nisoxetine (Fuller et al., 1979) was added throughout the experiment to avoid displacement of $\left[{ }^{3} \mathrm{H}\right]$ noradrenaline from its storage vesicles by 5-HT receptor agonists. The effect of electrically stimulating the slices preloaded with $\left[{ }^{3} \mathrm{H}\right]$ noradrenaline on the fractional release of tritium in the presence of nisoxetine is shown in Fig. 1A. The electrically evoked release of $\left[{ }^{3} \mathrm{H}\right]$ noradrenaline was considerably enhanced in the presence $\because \because$ a noradrenaline reuptake blocker (without nisoxetine: $S_{1}=1.47 \pm 0.12 c^{\prime} \cdot n=25$; with nisoxetine: $\left.S_{1}=3.42 \pm 0.27 c^{\circ}, n=25\right)$. However. the $S_{2} / S_{1}$ ratios were near unity and the $B_{3} / B_{1}$ ratios were approximately 0.8 , with or without nisoxetine in the superfusion medium.

When the 5-HT $\mathrm{H}_{3}$ receptor agonist 2-methyl-5-HT $(100 \mu \mathrm{M})$ was introduced $8 \mathrm{~min}$ before $S_{2}$, the electrically evoked release of [ ${ }^{3} \mathrm{H}$ ]noradrenaline elicited in $S_{2}$ was enhanced by $45 \%$ compared to that in $S_{1}$, without any change of the basal outflow (Fig. 1B). Concentration-effect curves were generated for hippocampus. hypothalamus and frontal cortex slices to compare the potentiating effect of 2-methyl-5-HT among these brain areas. The enhancing effect of 2-methyl-5-HT was concentration-dependent and similar in the hippocampus and hypothamalus (Fig. 2A and 2B). However, concentrations of 10 and $30 \mu \mathrm{M}$ of 2-methyl-5-HT did not change significantly the electrically evoked release of $\left[{ }^{3} \mathrm{H}\right]$ noradrenaline in frontal cortex slices. Nonetheless, $100 \mu \mathrm{M}$ of 2 -methyl-5-HT produced in this brain area an enhancement of the $\left[{ }^{3} \mathrm{H}\right]$ noradrenaline release of the same amplitude to that observed in the hippocampus and the hypothalamus (Fig. $2 \mathrm{C}$ ).

The enhancing effect of 2-methyl-5-HT $(100 \mu \mathrm{M})$ in hippocampus was blocked by the addition of a $5-\mathrm{HT}_{3}$ receptor antagonist, ondansetron $(1 \mu \mathrm{M})$, throughout 
the superfusion. In this condition. ondansetron did not change by inclf the $S_{z}, S_{1}$ ratio (Fig. 3 ).

Another 5-fit; receptor agonist. m-chorophenylbiguanide. Wals lested in the rat hippocimpus. At at concentration of $11.1 \mu . \mathrm{V}$. this drug added $8 \mathrm{~min}$ before $S_{2}$ did not change the eroked release of $\left[{ }^{3} \mathrm{H}\right]$ noradrenaline. but when the concentration was increased to 1 and to $\mu \mathrm{M}$. there was an increase in the eroked release of [' $H]$ noratdrenaline of $17 r^{\prime}$; and $42 r^{\prime} ;$. respectively (control: $S_{2} / S_{1}=1.01 \pm 0.05 . n=9$. $m$-chlorophenylbiguanide $0.1 \mu \mathrm{M}: S_{:} / S_{1}=1.08 \pm 0.0 \%, n=5$; $m$-chlorophenylbiguanide $1 \mu \mathrm{M}: \quad S_{2} / S_{1}=1.21 \pm 0.07$. $n=5, \quad P<0.05$ : m-chlorophenylbiguanide 10 $\mu \mathrm{M}$ :
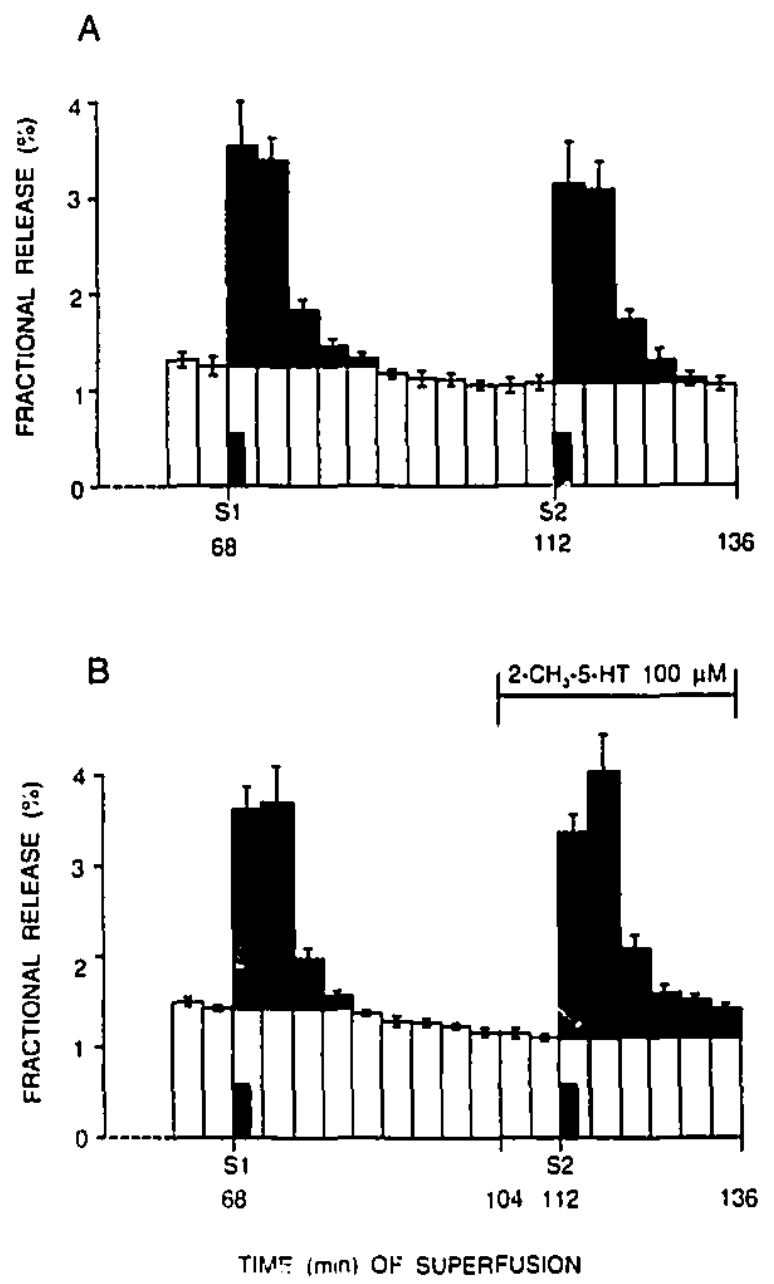

Fip. 1. Bassil and electrically esoked releast of tritium from hippoc:ampus slices preleaded with ['H] noradrenaline in a Krebs bulfer contaising $1 \mu .11$ of nisoxetine throughout superfusion in the control condition (A). and with the introduction of 100$) \mu \mathrm{M}$ of $2-$ methyl-5.HT $8 \mathrm{~min}$ before the second period of electrical stimulation (B). Each stimulation peried consisted of 36 (f) pulses delivered at an intensity of 20) $\mathrm{mA}$, with a durition of $2 \mathrm{~ms}$, and at a frequency of $3 \mathrm{~Hz}$. Ordinates represent the amount of tritium released per 4 min sample expressed as a fraction of the teral tiss a tritium content at the onset of the respective collection period. The hatched areiss represem the propurtion of tritium owerfluw ewoked by the stimulations. Dita are metans \pm S.E.M. of 5 experiments.
A

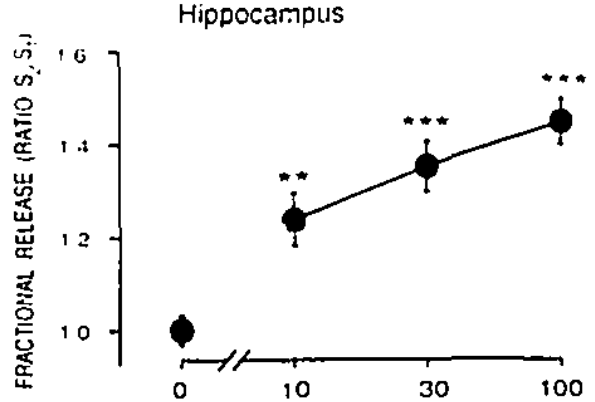

B

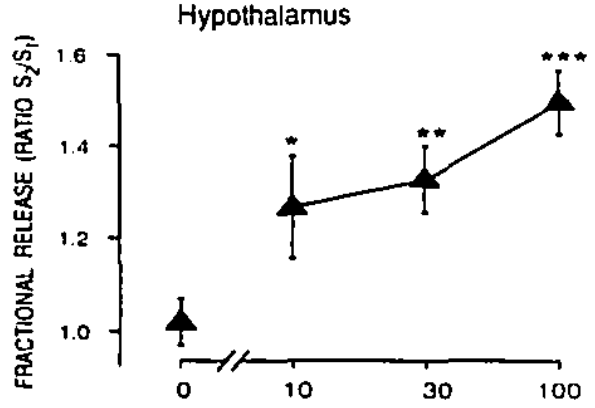

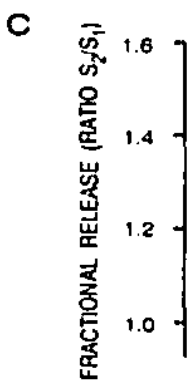

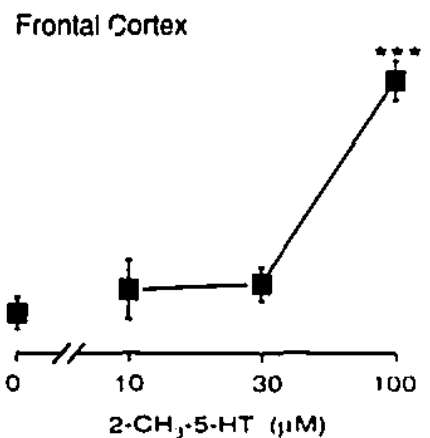

Fig. 2. Concentration-effect curies of 2 -methyl-5-1rr, introdected $x$ min hefore $S_{z}$ in a Krebs huffer containing $1 \mu$ il of nisuretine. on the relcatse of tritium elicited by the electrical itimulation if hip. pocampus (A). hypothalamus (B). and frontal corlex (C) vices preloaded with ['H]noradrenaline. Ordinate is the fratetion of torill lissue radioactivity released by 360 pulses $(20 \mathrm{~ms}$. $2 \mathrm{~ms}$. $3 \mathrm{H}, \mathrm{l})$ expressed as the ratio $\left(S_{2} / S_{1}\right)$ oblained hetseen the second peried of stimulation in the presence of 2 -methyl-5-11T $\left(S_{2}\right)$ and the tirst one done without this drug $(S I)$. Each point represents the mean t. S.E.M. of $5-10$ experiments. ${ }^{*} p<0.015 .{ }^{* *} p<0.011 .{ }^{* *} p$. 0.0111. using the two-tailed Student's :-test.

$\left.S_{2} / S_{1}=1.46 \pm 0.11, n=5 . \quad P<0.011\right)$, without any change in the basal outflow of raddioactivity. However. the enhancing effect of $10 \mu \mathrm{M}$ of $m$-chlorophenylbiguanide was not blocked by $0.1 \mu \mathrm{M}$ of $(S)$-zatopride nor by $1 \mu \mathrm{M}$ of ondansetron introduced before $S_{1}$ and maintained throughout superfusion (data not shown).

The agonist 2-methyl-5-HT was also introduced 20 $\min$ before $S_{2}$ to assess the possibility that the $5-\mathrm{HT}$ receptor involved could rapidly desensitize. The spontaneous outflow of radioactivity following the addition of 2-methyl-5-HT $20 \mathrm{~min}$ before $S_{2}\left(B_{2} / B_{1}=0.87 \pm\right.$ 


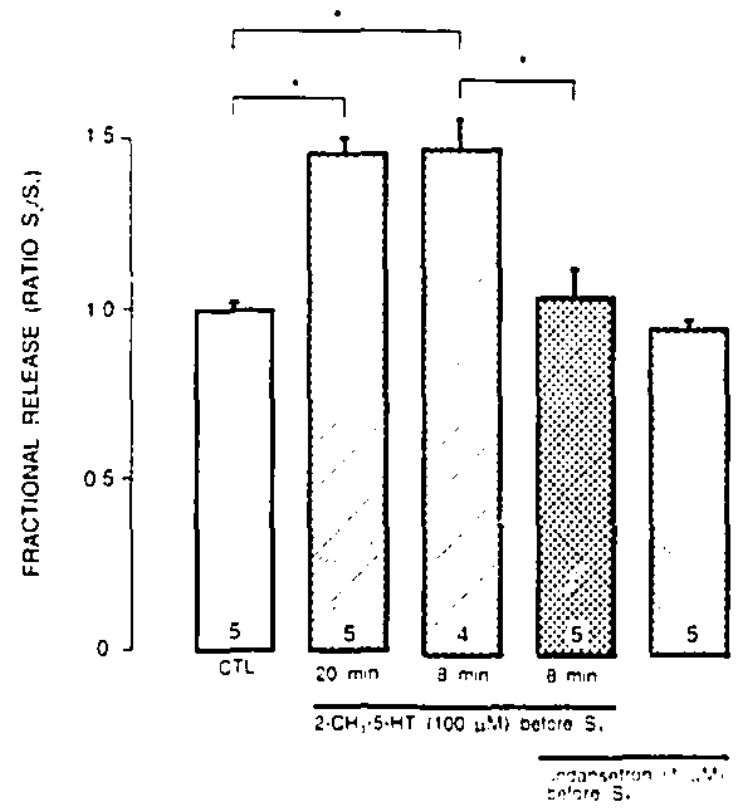

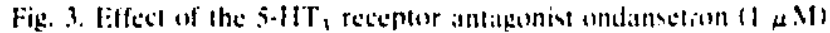

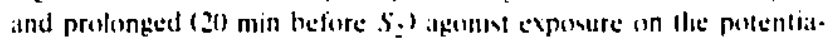
twon of the evoked [ 'H]nerradrenalline release induced by 2-methỵl-5.

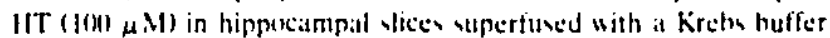
containing I $\mu \mathrm{M}$ nimexetine. Ondanectron wass present before $S_{1}$ and throughoul vunerfusion. Datil are means $\div$ S.E.M. The number of experiments is given at the lowtom al each column. " $P<10.01$. using the turblitiled student' tetest.

0.02. $n=5$ ) was not different from that observed following its addition 8 min before $S_{2}\left(B_{2} / B_{1}=0.81 \pm\right.$ 0.05. $n=4)$. Most importantly. tiis enhancing effect elicited by this $5 \cdot \mathrm{HT}_{3}$ receptor agonist introduced 20 min hefore $S_{2}$ did not differ from that produced by its addition $S$ min before $S_{2}$ (Fig. 3 ).

\subsection{Effect of iarious 5./HT recteptor agemists on the 'roked relectse' of tritiated noralre'naline'}

The 5-HT receptor agonists, 5-HT and 5.CT, were tested here at the highest concentration shown to be effective in the study of Feuersicin and Hertting (1986) in the rathit hippocampus. In our conditions. $30 \mu \mathrm{M}$ $5 \cdot H T$ introduced 8 min before $S_{2}$ enhanced by 23 ; the evoked release of [ ${ }^{3} \mathrm{H}$ ]noradrenaline without affecting basal tritium efflux, while 5-CT at the same concentration did not alter the $S_{3} / S_{1}$ ratio (Table 1). The 5-HT , receptor agonist 8.OH-DPAT $(0.1 \mu \mathrm{M}$ : Gozlan et al., 1983) and the 5-HT 183 receptor agonist CP-93.129 (10 $\mu \mathrm{M}$ : Macor et al. 1990) did not alter the eroked release of $\left[{ }^{3} \mathrm{H}\right]$ noradrenaline (Table 1$)$ Yet. 8-OH-DPAT and CP-93.129 at the same or lower concentrations efficiently decreased the release of S-HT by acting on somatodendritic or terminal autoreceptors (Hjorth and Tao, 1991: Skingle ot al.. 1992). On the other hand, the $5-H T_{3 N} ; C^{2}$ receptor agonist ( \pm )-DOI
T.Nile 1

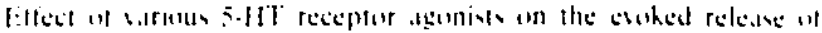

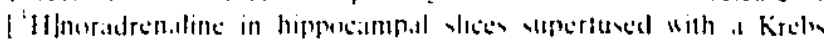

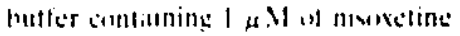

\begin{tabular}{|c|c|c|c|}
\hline Drit!̣ & $\begin{array}{l}\text { (ionicentrittun } \\
(\mu \mathrm{N})\end{array}$ & $n$ & Rutios s, s: \\
\hline Cinstrol & & 24 & $|f| \mid=11.112$ \\
\hline $51 \mathrm{HT}$ & . & 5 & $1.23+11.114 \cdot$ \\
\hline S. $\mathrm{T}$ & ill & $s$ & $1.11 .4-11.113$ \\
\hline$\therefore \cdot() \| \cdot D P A T$ & 0.1 & I1\} & $1.114=11.115$ \\
\hline$(19.4: 129$ & 111 & $\Sigma$ & ב $11.94=0.10$ \\
\hline $1+|-|)(3)$ & 1 & III & $1.119 \pm 11.12=$ \\
\hline
\end{tabular}

The agenists were introduced in the medium $s$ min hefore $S_{z}$, all igents remaining present in the supertusalte until the end of superfuvinn. Data are mean - S.E.MI.: $n=$ number of experiments per group.

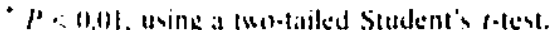

enhanced the evoked release of $\left[{ }^{3} \mathrm{H}\right]$ noradrenaline (Fig. 4). This effect. however, was not hlocked when $1 \mu . \mathrm{M}$ of the $5 \cdot \mathrm{HT}_{3}$ receptor antagonist ondansetron was introduced before $S_{1}$ and maintained throughout su. perfusion (data not shown). It was thus decmed out of the scope of the present study to investigate further this effect. Nonetheless, it is noteworthy that ( \pm )-DOI probably remained selective for $5-\mathrm{HT}_{2}$ and $5 \cdot \mathrm{HT}_{3}$ receptors at $1 \mu \mathrm{M}$ since this is under 100 -fold above $K_{\mathrm{d}}\left(5-\mathrm{HT}_{3 \mathrm{~N}}: p K_{\mathrm{d}}=7.8: 5-\mathrm{HT}_{3 \mathrm{C}}: \mathrm{p} K_{\mathrm{d}}=7.7:\right.$ Hover.

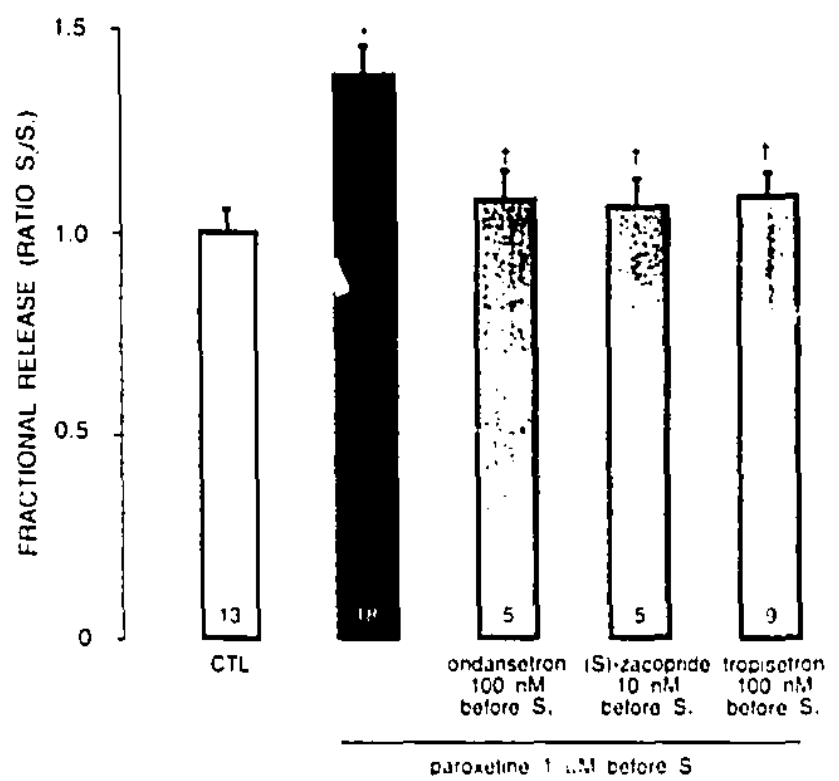

Fig. 4. The effect of pintoxetine $(1, \mu .11)$ on the esoked releatse of ('1])noradrenaline in hipporampal slices and its blockade by the s.11T, receptor antiagenists ondansetron (S)-akcopride and tronisetron. The antigenists were present hefore $S_{1}$ and throughout superfusion. Data atc meitns \pm S.E.M. The number of experiments is given at the hottom of each column. " $P<0.00)$, using a two-tailed Students petest comparing the paroxetine group with the control group. " $P<10,15$, using a twollated Student's totest comparing the 5-1IT, receptor antiggonists groups with the paroxetine group. 
1991) which is generilly considered ateceptable in release studies.

\subsection{Effect of paroxetine and 5-HT, receptor antagonists} on the eroked rele'ase of tritianed norradrenaline

The specific 5-HT reuptake inhibitor paroxetine was added to the medium to test whether an elevation in the synaptic availability of endogenous $5-\mathrm{HT}$ would alter the release of $\left[{ }^{3} \mathrm{H}\right]$ nor adrenaline. Paroxetine (1 $\mu M$ ) introduced 8 min before $S$, enhanced by $38 \%$ the electrically evoked release of $\left[{ }^{3} \mathrm{H}\right]$ noradrenaline from preloaded slices of hippocampus, without enhancing the basal outflow of tritium. This effect was blocked by adding throughout superfusion nanomolar concentrations of the 5-HT ${ }_{3}$ receptor antagonists, ondansetron. (S)-zacopride and tropisetron (Fig. 4).

The presence in the basal condition. of an endogenous tone of 5-HT on the 5- $\mathrm{HT}_{3}$ receptors modulating $\left[{ }^{3} \mathrm{H}\right]$ noradrenaline release, was tested with various concentrations of the $5 \cdot \mathrm{HT}_{3}$ receptor antagonists ondansetron, $(S)$-zacopride and tropisetron. None of these receptor antagonists produced any significant change in the spontaneous or the electrically croked release of $\left[{ }^{3} \mathrm{H}\right]$ noradrenaline at the concentrations tested, although there was a $15 \%$ decrease, albeit nonsignificant, in the evoked release with $100 \mathrm{nM}$ of $(S)$. zacopride (Table 2).

\subsection{Effect of 2-methyl-5.HT on $\alpha_{2} \cdot$ adrenergic autorecep- tors of noradrenaline terminals}

The $\alpha_{2}$-adrenoceptor antagonist potency of 2 methyl-5-HT was tested, in parallel with that of the $\alpha$-adrenoceptor antagonist idazoxan, against the inhibitory effect of the $\alpha_{2}$-adrenoceptor agonist UK14.304 on the evoked release of $\left[{ }^{3} \mathrm{H}\right]$ noradrenaline from preloaded slices of the rat hippocampus (Fig. 5). Since the presence of a noradrenaline reuptake blocker is required for the assessment of the effect of 2-methyl-

Table 2

Effect of the 5.11T, receptor intagenists, ondansetron. (S)-zateopride and trupistion on the evoked release of ['H]noradrenaline in hip. pocampal slices:

\begin{tabular}{llrl}
\hline Drug & $\begin{array}{l}\text { Concentration } \\
(\mu .1)\end{array}$ & $n$ & Ratio $S_{2} / S_{1}$ \\
\hline Control & & 10 & $1.02 \pm 0.07$ \\
Ondinsetron & 1 & 4 & $1.04 \pm 0.08$ \\
Zacopride & 0.01 & 4 & $1.05 \pm 0.06$ \\
& 0.1 & 4 & $0.85 \pm 0.03$ \\
Tropisetron & 0.1 & 4 & $1.06 \pm 0.05$
\end{tabular}

The antagonists were introduced in the medium 20 min before $S_{:}$. all agents remaining present in the superfusate untit the end of superfusion. Data are means \pm S.E.M.: $n$ a number of experiments per group.

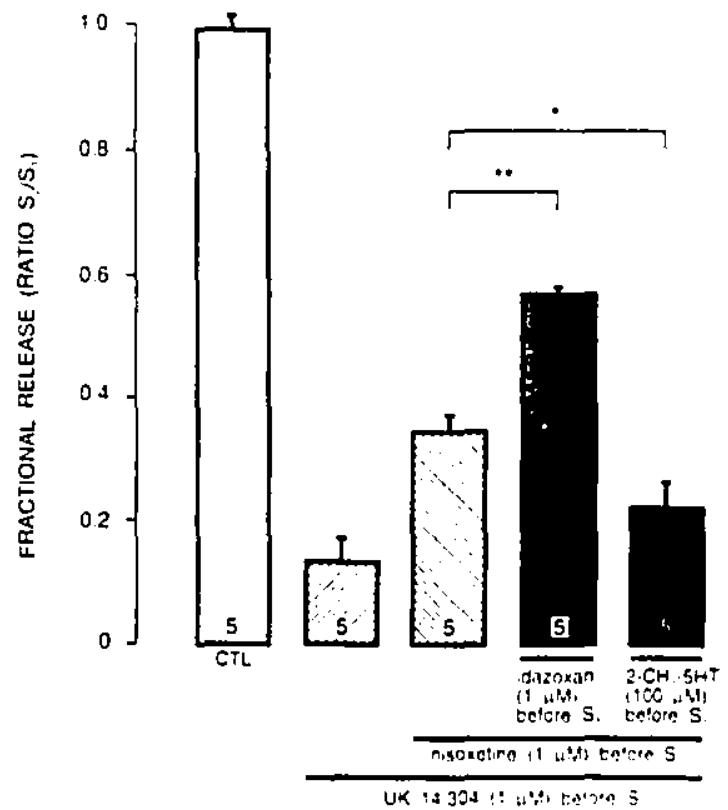

Fig. 5. Effect of idizoxan and 2-methỵ-5.HT un the L'K-14.31)4. mediated inhibition of the ewoked release of ['11]noradrenaline in hippocampal slices superfused with a Krebs huffer containing I $\mu . / 1$ of nioxetine. The az-idrenoceptor agonist UK-14.314 $(1, \mu . \mathrm{N})$ was intre duced in the medium 20 min before the second stimulation $\left(S_{2}\right)$ while the $a_{2}$-adrenoceptor antagunist idazoxin and the s-HT, ingenist 2-methyl-5.11T were introduced $20 \mathrm{~min}$ hefore the tirst vimulittion $\left(S_{1}\right)$, all agents rematining present in the superlusilte until the end of superfusion. Data are means \pm S.E.M. of tive evperimenls in each group. " $P<0.015 . " * P<0 .(0) 1$, using a Iwo-ladiled Students i-test.

5-HT, the inhibitory effect of UK-14.30.t was lested in the absence and in the presence of $1 \mu \mathrm{M}$ of nisoxetine. As expected, elevation of noradrenaline in the bio. phase induced by nisoxetine decreased the efficacy of UK-14.304 introduced $20 \mathrm{~min}$ before $S_{2}$ to inthibit the evoked release of $\left[{ }^{3} \mathrm{H}\right]$ noradrenaline (Fig. 5). Compared to control $S_{2} / S_{1}$ ratios, $1 \mu \mathrm{M}$ of UK-14.31)4 produced $83 \%$ and $70 \%$ inhibition in the absence and in the presence of nisoxetine. respectively.

The evoked release of $\left[{ }^{3} \mathrm{H}\right]$ noradrenaline was increased in the presence of $1 \mu \mathrm{M}$ of idazoxan (control: $S_{1}=3.0 \pm 0.2 \%, n=5$ idazoxan: $S_{1}=14.6 \pm 1.8 \%, n$ $=5$ ) as well as in the presence of 100$) \mu \mathrm{M}$ of $2-\mathrm{methyl-}$ 5-HT but to a much lesser degree (control: $S_{1}=2.1 \pm$ $0.2 \%, n=5 ; 2$-methyl-5-HT: $S_{1}=3.1 \pm 0.1 \%, n=5$ ). When $1 \mu \mathrm{M}$ idazoxan was added $20 \mathrm{~min}$ before $S_{1}$ until the end of the superfusion. the $S_{2} / S_{1}$ ratio wals $0.86 \pm 0.06(n=5)$. The addition of UK-14.304 inhibited by only $34 C^{3}$ the evoked release of $\left[{ }^{3} \mathrm{H}\right]$ noradrenaline in the presence of idazoxan, indicating an effective block of the $\alpha_{2}$-idrenergic autoreceptors. The presence of 2-methyl-5-HT in the same condition did not attenuate the inhibition by UK-14,304 of the evoked release of $\left[{ }^{3} \mathrm{H}\right]$ noradrenaline. In fact, the inhibition produced by UK-14.304 was slightly increased from 
$70 \%$ to sis? (hatsal ratio with 2-methyl-5-HT added hefore $S_{1}$ and present throughout: $S_{2} / S_{1}=1.13 \div 1$ i.iic. $n=5)$.

The 2-methyl-5-HT was also tested in the condition where there is no autoinhibition of noradrenaline iclabse through a $x_{z}$-adrenergic atuturcepters. Previous studies have shown that olectrical stimulations using $f$ pulse's at 1100 $\mathrm{Hz}$ (psetudo-(onc-pulse) elicit no autoinhibition of noradrenaline releatse. esen in the presence of a noradrenaline reuptake inhibitor (Zier et al.. 19sis). These results could be replicated in ur superfusion system. In the standard conditions (stimulation at i $\mathrm{Hz}$ during $2 \mathrm{~min}$ in the presence of $1 \mu \mathrm{M}$ of nisoxetine). 1 $\mu \mathrm{M}$ of the a $x_{2}-$ addrenoceptor antagonist idazoxan added 20 $\mathrm{min}$ before $S$, produced a 301$)^{\circ}$; increatse in the evoked release of ['H]noradrenaline (control: $S_{2} / S_{1}=$ $0.94 \pm 0.06, n=4:$ idazoxan: $S, / S_{1}=3.80 \pm 0.10 . n=$ 4). When the pseudo-one-pulse stimulations were used (in the presence of $1 \mu \mathrm{M}$ of nisoxetine) the evoked release of $\left[{ }^{3} H\right]$ noradrenaline $\left(S_{1}\right)$ was on average 0. ht $\pm 0.05 \%(n=28)$, and the introduction of $1 \mu . \mathrm{M}$ of idazoxan 20 min before $S$, did not facilitate the ewoked release (control: $S_{2} / S_{1}=1.16 \pm 0.14, n=6$ : idazoxian: $S_{2} / S_{1}=1.19 \pm(0.10, n=5)$. In contrast. the presence of $1 \mu \mathrm{M}$ of the $x_{2}$-aldrenoceptor agonist UK-14.3014 20 min before the second pseudo-one-pulse produced its usual inhibitory effect fontrol: $S_{2} / S_{1}=0.86=0.0 \mathrm{~s}, n$ $=5$ : UK-14.3(1): $S_{2} / S_{1}=(0.04 \pm 1) .02$. $\left.n=4\right)$. Most importantly. $100 \mu \mathrm{M}$ of 2-methyl-5-HT introduced $s \mathrm{~min}$ hefore the second pecudoesne-pulse produced a $75 \%$; enhancement of the eroked release of [ $\left[{ }^{3} \mathrm{H}\right]$ noradrena-

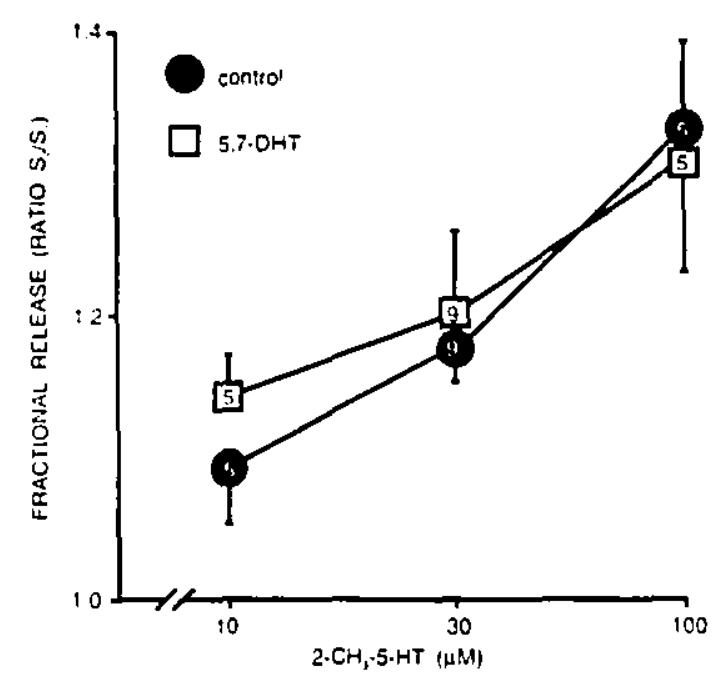

Fig. 6. Effect of 5.7.DHT lesiom of the 5-HT fihers on the 2-melhyl5-HT-medialled enlsancement of the cwoked releise of $($ 'HI. noradrenaline in hippeximpal slices superlused with a krehs huller containitg $1 \mu \mathrm{M}$ of nisoxetine. The toxin 5.7.DHT was injected i.c... as well as injected in the dorsal and the median raphe 3 weths before the experiments. Data are meim $=$ S.E.A1. The number of experiment.s is gisen within each data point. line combrol: $s, / S_{1}=0.71 \pm 11.117, n=4: 2 \cdot$ medlyl-s. HT: $\left.5: S_{1}=1.24=11.11, n=4: P<11.111\right)$.

3.5. Effert of lesioming 5-HT Meturoms on the enhancic-

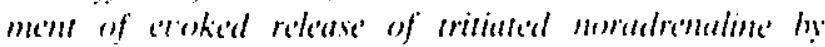
$2-11+1 / n \% .5-11 T$

Tike lesioning of 5.HT neurons with the neuromin 5.7-DHT Was performed to assess whether the 2methyl-5-HT-mediated enhancemen: of the esoked release of [ H]noradrenaline was mediated by 5-HT neurons. The results of Fig. 6 show that the effect of 2-methyl-5-HT was still present in 5.7-DHT-pretreated rats. Furthermore, a shift to the left of the concentrition-response curse of 2-methyl-5-HT would have been expected if the 5-HT receptors involved had hecome supersensitive in response to the prolonged $5-H T$ depletion induced by the lesions. The responsiteness of tike 5.HT: receptors modulating ["H]noradrenaline release was not enhanced since the concentration-effect relationship was unaltered (Fig. 6).

\section{Discussion}

The present study demonstrates that 5-11T: receptors facilitate noradrenaline release in various limbic structures of the rat brain, namely, the hippocianpus. the hypothalamus and the frontal cortex. These results ar: consistent with a previous study hy Feucrstein and Herting $(1986)$ in the rabbit hippocimpus. The facilitatory effect of 5-HT receptor agonists on the electrically evoled relcase of [ ${ }^{3} \mathrm{H}$ ]noradrenaline reported hy these athors was then attributed to the attivation of 5-HT-M receptors. It is now generally accepted that $5 \cdot H T \cdot M$ and $5-H_{3}$ are (wo denominations for the sime receptor (Hoyer. 1990). However, the enhancement of the esoked release of $\left[{ }^{3} H\right]$ noradrenaline from preloaded slices of rabbit hippocampus by 5-CT in Feuerstein and Herttings study cannot be alcounted for by $5 . H T_{3}$ receptors since the $p K_{3}$ of $5-C T$ for $5-\mathrm{HT}$ : recepters is only 4.1 (Hoyer. 194)1). In the present study the introduction of $5-C T$ intu the superfusion medium $\&$ min before the second stimulation did not alter the evoked release of [ $\mathrm{H}$ ]noradrenaline from rat hippocampus slices (Table 1). At present. we have no definitive explanation for this discrepancy except for a possible species difference. At any rate, on the hasis of the negative result ootained here with 5.C"T. ats well ats those obtained with 8 -OH-DPAT and CP-93,124) ("Talble 1), an implication of 5-HT, receptors in the modulation of noradrenaline release in the rat hippocampus can be ruled out. Finally, an enhansesinent in the evoked release of $\left[{ }^{3} H\right]$ noradrenaline was also found with $( \pm)-\mathrm{DOI}$ which is a $5 \cdot \mathrm{HT}_{21} \ldots$ ligand (Tathle 1 ). 
The response is probibly not mediated by 5-HT:C. receptors since it was not obs: ved with $30 \mu . \mathrm{M}$ 5-CT. which hats moderate affinity for this site $\left(\mathrm{pK}_{4}=6.2\right.$ : Hoyer. l990). Since ondansetron did not block this effect of $( \pm)$-DOI. thus excluding any involiement of the 5.HT: recepturs. it was deemed out of the scope of the present study to further characterize this response. Nonetheless, it is noteworthy that the effect of $( \pm)$-DOI in the present study does not concord with the results of a recent in vivo microdialysis study where the administration of this drug decreased the release of endogenous noradrenaline in the same brain area (Done and Sharp. 1942).

An enhancement in $\left[{ }^{3} \mathrm{H}\right]$ noradrenaline release was atso observed with the $5 \cdot \mathrm{HT}_{3}$ agonist $m$-chlorophenylbiguanide. However, considering that this effect was not blocked by ondansetron nor by $(S)$-zacopride and that micromolar concentrations of this potent $5-\mathrm{HT}_{3}$ receptor agonist $\left(\left[{ }^{3} \mathrm{H}\right] \mathrm{GR} 67330\right.$ binding $\mathrm{p} K_{\mathrm{i}}=9.5$; Kilpatrick et al.. 1990) were required. m-chlorophenylbiguanide at high concentrations probably does not act through $5-\mathrm{HT}_{3}$ receptors to increase $\left[{ }^{3} \mathrm{H}\right.$ ]noradrenaline release. It was intriguing that nanomolar concentrations of $r$-chlorophenylbiguanide failed to produce any enhancement of [ $\left.{ }^{3} \mathrm{H}\right]$ noradrenaline release similar to that of $5-\mathrm{HT}$ and 2-methyl-5-HT. A previous report by Kilpatrick et al. (1990) has shown that nanomolar concentrations of $m$-chlorophenylbiguanide depolarize rat vagus nerve through $5-\mathrm{HT}_{3}$ receptors, an effect which is blocked by the 5-HT, receptor antagonist ondansetron. Furthermore. the enhancing effect of phenylbiguanide on dopamine release in the rat nucleus accumbens is blocked by zacopride and ondansetron (Chen et al., 1991). However, $m$-chlorophenylbiguanide was found to be ineffective when tested on 5-HT ${ }_{3}$ receptors mediating fast excitatory synaptic potential in the rat amygdala (Sugita et al., 1992). In addition. Steward et al. (1993) recently reported that $\left[{ }^{3} \mathrm{H}\right] \mathrm{m}$-chlorophenylbiguanide displays specific binding in hindbrain nuclei of the rat (nucleus tractus solitarius. area postrema and dorsal motor nucleus of the vagus nerve) similarly to other $5-\mathrm{HT}_{3}$ ligands such as $\left[{ }^{3} \mathrm{H}\right](S)$-zacopride. But, in contrast to $\left[{ }^{3} \mathrm{H}\right](S)$ zacopride. $\left[{ }^{3} \mathrm{H}\right] \mathrm{m}$-chlorophenylbiguanide did not display any specific binding in forebrain areas of the rat (e.g. cerebral cortex, hippocampus, amygdala). Hence, further studies are required to determine whether there is intraspecies heterogeneity of $5-\mathrm{HT}_{3}$ receptors, as it may appear from the above.

The present finding of an enhancement of the electrically evoked release of $\left[{ }^{3} \mathrm{H}\right]$ noradrenaline in the rat hypothalamus by 2-methyl-5-HT is discordant with the results of Blandina et al. (1991) who found the opposite with potassium-evoked release of endogenous noradrenaline in the same brain region of this species. Three possibilities can be envisaged to account for this discrepancy. First. they used potassium while we used clectrical pulses to evole the release of neurotransmitter. Second. contrary to the release of preloaded tritiated neurotransmitters. the release of endogenous neurotransmitters reflects the contribution of newly synthesized neurotransmitter. However, although these wo factors could account for a quantitative difference in the amplitude of 2-methyl-5-HT modulation, they can hardly explain a qualitative difference, i.e. an inhihition instead of a facilitation. Another difference that could account for the discrepancy is the fact that the monoamine oxidase inhibitor pargyline was used in the experiments of Blandina et al. (1991) to prevent the degradation of noradrenaline. Pargyline prevents not only the degradation of noradrenaline, but also that of 5-HT and dopamine and consequently elevates the concentration of these neurotransmitters in the biophase. Thus, this concurrent and sustained elevation of the levels of 5-HT, noradrenaline, and dopamine may have contributed to decrease the evoked release of endogenous noradrenaline. It would thus be interesting to study the $5-\mathrm{HT}_{3}$ modulation of endogenous noradrenaline release using a selective noradrenaline reuptake blocker rather than a monoamine oxidase inhibitor.

It was conceivable that the enhancing effect of 2methyl-5-HT in the present study cosild have been due to an antagonistic action of this drug at $\alpha_{z}$-adrenergic autoreceptors given the negative feedback role they exert on noradrenaline release. It was thus deemed crucial to test this possibility. The effect of UK-14.31)4 and idazoxan were tested in the presence of nisoxetine. The smaller effects of these $\alpha_{2}$-ildrenergic agents in the presence of nisoxetine. compared to those in the control condition. most likely resulted from a competition with the increased endogenous noradrenaline in the biophase induced by noradienaline reuptake blockade. Nonetheless, the results clearly indicate the inability of 2-methyl-5-HT, contrarily to idazoxan, to block the agonistic effect of UK-14,304 at terminal $\alpha_{2}$-autoreceptors. In fact, the inhibitory action of UK-14,304 on $\left[{ }^{3} \mathrm{H}\right]$ noradrenaline release was even greater in the presence of 2-methyl-5-HT. This enhancement of the effect of UK-14,304 could possibly be related to the increased release of $\left[{ }^{3} \mathrm{H}\right]$ noradrenaline in the presence of 2-methyl-5-HT which could have allowed a greater amplitude of negative modulation. A second argument against an antagonistic action of 2-methyl-5-HT att $\alpha_{2}$ autoreceptors is the fact that this drug still produced its enhancing effect in an autoinhibition-free environment using pseudo-one-pulse experiments.

Although the experiments with the selective 5-HT reuptake blocker paroxetine clearly indicate an activation of $5-\mathrm{HT}_{3}$ receptors by elevated levels of endogenous 5-HT, a tonic action of this neurotransmitter was not apparent under basal conditions. Indeed. the 5-HT, 
receptor antagonists ondansetron, $(S)$-zacopride and tropisetron did not decrease by themselves the evoked release of $\left[{ }^{3} \mathrm{H}\right]$ noradrenaline (Table 2). However, one should be cautious in interpreting a lack of apparent endogenous tone from results obtained in vitro. For instance, in vivo results indicating an endogenous tone of noradrenaline on $\alpha_{2}$-adrenergic heteroreceptors on 5-HT terminals are at variance with in vitro studies reporting either a weak or no endogenous tone at this level (see Mongeau et al.. 1993 for a review).

The question of whether the responsiveness of the $5-\mathrm{HT}_{3}$ receptors modulating the evoked release of $\left[{ }^{3} \mathrm{H}\right]$ noradrenaline decreased following prolonged agonist stimulation was also considered. Many reports have indicated the occurrence of a rapid desensitization of $5 \cdot \mathrm{HT}_{3}$ receptors in different preparations (Fozard et al., 1979; Ireland and Tyers, 1987; Yakel and Jackson, 1988; Sugita et al., 1992). In the guinea pig frontal cortex and hypothalamus. 2-methyl-5-HT was reported to enhance the evoked release of $\left[{ }^{3} \mathrm{H}\right] 5$ HT from preloaded slices when introduced in the medium $8 \mathrm{~min}$, but not when added $20 \mathrm{~min}$, before the second stimulation period (Galzin and Langer. 1991; Blier and Bouchard, 1993). The agonist 2-methyl-5-HT was thus tested under identical conditions. No decrement in its effectiveness in increasing $\left[{ }^{3} \mathrm{H}\right]$ noradrenaline release was observed after $20 \mathrm{~min}$ of exposure compared to the $8 \mathrm{~min}$ exposure. indicating that a desensitization does not occur within this time period. It is noteworthy that a similar absence of desensitization of 5-HT, receptors has been reported in the modulation of dopamine release in the rat striatum (Blandina et al., 1989). Thus, it might be argued that the $5 \cdot \mathrm{HT}_{3}$ receptors modulating the release of catecholamines are different, albeit maybe only slightly so, from those modulating 5-HT release. Indeed. Yakel et al. (1992) using cloned $5 \cdot \mathrm{HT}_{3}$ receptors have shown that a single amino acid substitution is sufficient to produce marked changes in desensitization kinetics. Alternatively, the differences between the two receptors might be explained by intracellular factors. For instance, $5-\mathrm{HT}_{3}$ receptor desensitization is accelerated in the presence of agents, such as the adenylate cyclase activator forskolin or the phosphodiesterase inhibitor isobutylmethylxanthine, which increase cAMP levels (Yakel, 1992).

Considering that the activation of the $5-\mathrm{HT}_{3}$ receptors enhances the release of 5-HT (Galzin and Langer, 1991; Blier and Bouchard, 1993), it was theoretically possible that the enhanced evoked release of $\left[{ }^{3} \mathrm{H}\right]$ noradrenaline observed herein following exposure to $5-\mathrm{HT}_{3}$ receptor agonists was indirectly mediated by 5-HT neurons. This eventuality was thus tested by lesioning 5-HT neurons with the neurotoxin 5,7-DHT. No change in the potency of 2-methyl-5-HT to enhance the evoked release of $\left[{ }^{3} \mathrm{H}\right]$ noradrenaline was observed in the 5.7-DHT-pretreated rats. indicating that 5-HT fibers are not necessary for the occurrence of this effect. Similar results were also ubtained for the $5-\mathrm{HT}_{3}$ receptors that modulate the release of dopamine (Chen et al.. 1991). A further objective of the 5.7-DHT lesions was to assess whether the $5-\mathrm{HT}_{3}$ receptors modulating $\left[{ }^{3} \mathrm{H}\right]$ noradrenaline release become supersensitive following long-term 5-HT depletion. Microiontophoretic applications of 5-HT and 2-methyl-5-HT produce a suppressant effect on the firing activity of prefrontal cortex pyramidal neurons (Wang et al., 1992). The current-response of this effect was shown to be shifted to the left after 5,7-DHT lesions, indicating a supersensitivity of the $5 \cdot \mathrm{HT}_{3}$ receptors following chemical denervation of the 5-HT fibers (Wang et al.. 1992). Such an adaptive phenomenon was not observed for the $5-\mathrm{HT}_{3}$ receptors that modulate the release of $\left[{ }^{3} \mathrm{H}\right]$ noradrenaline after a similar denervation (Fig. 6). The reason might be that the endogenous tone of 5.HT on these receptors is rather weak or absent, as discussed above.

Although the results of the present study show that the $5-\mathrm{HT}_{3}$ receptors which modulate $\left[{ }^{3} \mathrm{H}\right.$ ]noradrenaline release are not located on $5-H T$, no information is yet available about the localization of these receptors. A recent report (Kidd et al.. 1993) indicates that the binding of $\left[{ }^{3} \mathrm{H}\right](S)$-zacopride to $5-\mathrm{HT}_{3}$ receptors is not affected by a noradrenaline denervation. suggesting that the effects of $5-\mathrm{HT}_{3}$ ligands on noradrenaline release are not attributable to heteroreceptors on noradrenergic neurons. However. only experiments using isolated noradrenergic nerve endings (i.e. in synaptosomes) could reliably resolve this issue.

In conclusion, the presence of facilitatory $5 \cdot \mathrm{HT}_{3}$ receptors modulating the release of noradrenaline in the rat hippocampus, frontal cortex and hypothalamus was documented. Physiological relevance of these results obtained with 2-methyl-5-HT and exogenous 5-HT was indicated by the demonstration that endogenous 5-HT, obtained by blocking 5-HT reuptake, can elicit a facilitating effect on $5-\mathrm{HT}_{3}$ receptors. The responsiveness of these receptors is not affected by short-term agonist exposure, nor by long-term 5-HT depletion. It would be interesting to determine if the sensitivity of these $5-\mathrm{HT}_{3}$ receptors is altered by long-term antidepressant treatments that increase the synaptic availability of 5-HT.

\section{Acknowledgements}

This work was supported in part by the Medical Research Concil of Canada (MRC) grants MT.6hlt4 and 11014 and the Fonds de la Recherche en Sante du Québec. R.M. received a Studentship and P.B. a Scholarship from MRC. 


\section{References}

Aghajanian, G.K.. 19kil. Mescaline and LSD facilitate the activation of locus coeruleus neurons hy peripheral stimuli. Brain Res. 186. 442.

Blandinal. P.. J. Cinddfard and J.P. Green. 1988, Activation of a 5-HT, receptor releases dopamine from rat striatal slice. Eur. J. Pharmacol. 155, 349.

Blandinal. P.. 3. Goldfarh. B. Craddock-Royal and J.P. Green. I9s9. Release of endegenous dopimine by stimulation of 5-hydroxyinplaminc, receptors in rit striatum. J. Pharmacol. Exp. Ther. 251.803 .3$.

Blandina. P.. J. Goldfarb, J. Walcott and J.P. Green. 1991, Serolon. ergic modulation of the reledse of endogenous norepinephrine from rat hypothalamic slices. J. Pharmacol. Exp. Ther. 256. 341 .

Blier. P. and C. Buuchard. 199.3. Functional characterizattion of a $5-\mathrm{HT}_{3}$ receptor which modulates the release of $5 \cdot \mathrm{HT}$ in the guinea pig brain. Br. J. Pharmacol. 108, 13.

Bubker. D.H. and J.T. Williams, 1989. Serotonin agonists inhibit synaptic potentials in the rat locus ceruleus in vitro via S-hydroxytryptamine 1 and 5-hydroxytryptamine, ato receptors. J. Pharmacol. Exp. Ther. 250, 37.

Chen. J., H.M. Van Praag and E.L. Gardner. 1991. Activation of 5.HT, receptor by 1-phenylbiguanide increases dopamine release in the rat nucleus accumbens. Brain Res. 543. 354.

Chen. J.. W. Paredes. H.M. Van Praag. J.H. Lowinson and E.L. Gardner. 1992. Presynaptic dopamine release is enhanced by 5-HT, receptor activation in medial prefrontal costex of freely moving rats. Synapse 10, 264.

Chiang. C. and G. Aston-Jones. 1993. A 5-hydroxytryptamine, agonits augments $\gamma$-aminobutyric acid and excitatory amino acid inputs to noradrenergic locus coeruleus neurons. Neuroscience 5., 4199.

Done. C.J.G. and T. Sharp. 1992. Evidence that 5-HT, receptor activation decreases noradrenaline release in rat hippocampus in vivo. Br. J. Pharmacol. 107, 240.

Feuerstein. T.J. and G. Hertting. 1986, Serotonin (5-HT) enhances hippocampal noradrenaline (NA) release: evidence for facilita. onn 5-HT receptors within the CNS. Natunyn-Schmied. Arch. Pharmacil. 333. 191.

Fozard. J.R.. 1984, Neuronal 5-HT receptors in the periphery, Neuropharmacology 23, 1473.

Fozard. J.R.. A.T.M. Mubarok .'li and G. Newgrosh. 1979, Blockade of serotonin receptors on autonomic neurones by $(-)$ cocaine and some related compounds, Eur. J. Pharmacol. 59. 195.

Fuller, R.W.. H.D. Snoddy and K.W. Perry, 1979, Nisoxetine antagonism of norepinephrine depletion in brain and heart after alphamethyl-m-tyrosine administration. Neuropharmacology 18.767.

Galzin, A.M. and S.Z. Langer, 1991. Modulation of 5.HT release by presynaptic inhibitory and facilitatory 5-HT receptors in brain slices. Adv. Biosci. 82, 59.

Göthert. M.. P. Kollecker, N. Rohm and H.-R. Zerkowski, I986. Inhibitory presynaptic 5-hydroxytryptamine (5-HT) receptors on the symputhetic nerves of the human saphenous vein. NaunynSchmied. Arch. Pharmacol. 332, 317.

Gozlan. H.. S. El Mestikawy, L. Pichat, J. Glowinski and M. Hamon. 1983. Identification of presynaptic serotonin alltoreceptors using a new ligand "H.PAT. Nature 305. 140.

Hammond. D.L.. G.M. Tyce and T.L. Yaksh, 1985. Efflux of 5-hy. droxytryptamine and noradrenaline into spinal cord superfusates during stimulation of the rat medulla, J. Physiol. (London) 359. 151.

Hjorth, S. and R. Talo, 1991. The putative 5-HT $1 \mathrm{a}$ receptor atgunist CP.93,129 suppresses rat hippocampal 5.HT release in vivo: comparison with RU 24969. Eur. J. Pharmacol. 209. 249.
Hoyer. D., 1990, Serotonin, 5-HT, 5-HT, and 5-HT-M receptors. Neuropsychopharmacology 3. 371 .

Hoyer. D.. 1991. The 5.HT receptor family: ligands, distribution and receptor-tfector coupling. in: 5-HT $\mathrm{HA}_{\mathrm{A}}$ Agonists. 5.HT, Antagonists and Benzodiazepines: Their Comparative Behavioral Phar. mucology. eds. R.J. Rodgers and S.J. Cooper (Wiley. Chichester) p. 31 .

Irelind. S.J. and M.B. Tyers. 1987. Pharmacological characterisation of 5-HT-induced depolarisation of the rat isolated vagus nerve. Br. J. Pharmacol. 9), 229.

Jiang, L.H. C.R. Ashby, Jr. R.J. Kasser and R.Y. Wang. 1990), The iffect of intraventriculas administration of the $5-\mathrm{HT}_{3}$ receptor agonist 2-meihylserotonin on the release of dopamine in the nucleus accumbens: an in vivo chronocoulometric study. Brain Res. 513. 156.

Kidd, E.J.. A.M. Laporte, X. Langlois, C.-M. Fattaccini, C. Doyen, M.C. Lombard. H. Gozian and M. Hamon. 1993, 5-HT, receptors in the rat centrall newous system are mainly located on nerve fibres and terminals. Brain Res. 612, 289.

Kilpatrick. G.J.. A. Butler. J. Burridge and A.W. Oxford. 1990, [-(m-Chlorophenyl)-biguanide, a potent high affinity $5 \cdot \mathrm{HT}_{3}$ receptor agonist, Eur. J. Phormacol. 182. 193.

Lechin F., B. Van der Dijs, J. Amat and M. Lechin. 19k9, Neu. roanatomical basis. in: Neurochemistry and Clinical Disorders: Circuitry of Some Psychiatric and Psychosomatic Syndromes, eds. F. Lechin and B. Van der Dijs (CRC Press, Boca Ritun) p. 1.

Macor, J.E., C.A. Burkhart, J.H. Heym. J.L. Ives, L.A. Lebel, M.E Newman. J.A. Nielsen, K. Ryan, D.W. Schulz, L.K. Torgersen and B.K. Koe. 1990, 3-(1,2,5,6-Tetrahydropyrid-4-yl)pysrololl 3,2b]pyrid-5-one: a potent and sclective serotonin (5-11 18 ) agonist and rotationally restricted phenulic analogue of 5 -methoxy-3. (1.2.5.6.tetranydropyrid-4-y!)indole, 3. Med. Chem. 33, 2087.

Methan, A.G. and D.L. Kreulen, 1991. Electrophysiological studies on the interaction of 5-hydroxytryptamine with sympathetic trans. mission in the guinea pig inferior mesenteric artery and panglion. J. Pharmacol. Exp. Ther. 256. 82.

Molderings. G.J., K. Fink. E. Schlicker and M. Gïthert. 1987. Inhibition of noradrenaline release via presynaptic 5-HTT 13 recep. tors in the rat vena cava, Naunyn-Schmied. Arch. Pharmacol. 336. 245.

Molderings. G.J., M. Göthert, K. Fink. E. Roth and E. Schlicker. 1989. Inhibition of noradrenaline release in the pig coronary artery via a novel serotonin receptor. Eur. J. Pharmacol. 164. 213.

Molderings, G.J., K. Werner. J. Likungu and M. Göthert. 1990, Inhibition of noradrenaline release irem the sympathetic nenes of the human saphenous vein via presynaptic 5.HT receptors similar to the $5-\mathrm{HT}_{1 \mathrm{I}}$ subtype. Naunyn-Schmied. Arch. Pharmacol. 342, 371 .

Mongtau. R., P. Blitr and C. De Montigny, 1993. In vivo electrophysiological evidence for a tonic inhibitory action of endogenous noradrenaline on $\alpha_{2}$-idrenetgic heleroreceptors of 5 -hydroxytryptamine terminals in the rat hippocampus. Naunyn-Schmied. Arch. Pharmacol. 347. 266.

Rasmussen, K., R.A. Glennon and G.K. Aghajanian, 1986. Phenethylamine hallucinogens in the locus coeruleus: potency of action correlates with rank order of 5-HT, binding affinity, Eur. J. Pharmacul. 132, 79.

Reimann. W. and F. Schneider, 1993, The serotonin receptor agonist 5 -methoxy- $N$. N-dimethyltryptamine facilitates noradrenaline release from rat spinal cord slices ard inhibits monoamine oxidase activity. Gen. Pharmacol. 24. 449.

Skingle. M.. A.J. Sleight and D.J. Rosser, 1992. Effect of local infusions of 8-OH-DPAT on extracellular levels of 5.HT in the anaesthetised guinea pig. Soc. Neurosci. Abstr. $18,46.4$.

Steward, L.J., K.E. West, G.J. Kilpatrick and N.M. Barnes, 1993, 
Labelling of $5 \cdot \mathrm{HT}_{3}$ receptor recognition sites in the rat brain using the agonist radioligand $\left[{ }^{3} \mathrm{H}\right]$ meta-chlorophenylbiguanide. Eur. J. Pharmacol. 243, 13.

Sugita. S., K.-Z. Shen and R.A. North, 1992. 5-Hydroxytryptamine is a fast excitatory transmitter at $5 \cdot \mathrm{HT}_{3}$ receptors in rat amygdala, Neuron $8,199$.

Wang. R.Y., J.Y. Zhang and C.R. Asthy, 1992. The intractrebroventricular administration of 5.7-dihydroxytryptamine (5.7-DHT) produces a functional supersensitivity of 5- $\mathrm{HT}_{3}$, but not 5-HT, or S-HT $T_{1 \lambda}$ receptors in the rat medial prefrontal cortex (mPFc): iontophoretic studies, in: Serotonin: from Cell Biology to Phar. macology and Therapeutics, Abstr. 2. 37.

Yakel. J.L., 1992. 5.HT, receptors as cotion channels. in: Central and Peripheral 5-HT, Receptors, ed. M. Hamon (Academic Press, London) p. 102.

Yakel. J.L. and M.B. Jackson. 1988, 5-HT, recepturs mediate rapid responses in cultured hippocampus and clonal cell line. Neuron 615,615 .

Yakel, J.L., A. Lagrulta. J.P. Adelman and R.A. North. 1993. Single amino acid substitution affects desensitizatio" of the 5-hydroxy. tryptamine type 3 receptor expressed in Kenopus oocytes, Proc. Natl. Acad. Sci. USA (9), 5030.

Zier, G., H. Drobny. B. Valenta anci E.A. Singer. 198k. Evidence against a functional link between noradrenaline uptake mechanisms and presynaptic $\alpha_{2}$-adrenoceptors. Naunyn-Schmied. Arch. Pharmacol. 337. 118. 


\section{Fifth article}

The 5-HT3 receptors that enhance the release of [3H]NA being fully characterized in the rat hippocampus, it was possible to assess the effect of long-term antidepressant treatments on the sensitivity of these receptors. It is noteworthy that this is the first study that has ever addressed this issue. Concentration-effect curves with the agonist 2-methyl5-HT were generated to assess the sensitivity of these 5-HT3 receptors following longterm treatments vith antidepressant drugs of various classes: a reversible type A MAOI, selective NA or 5-HT reuptake inhibitors, and TCAs. Our working hypothesis here was that long-term treatments with antidepressant drugs that increase the synaptic concentration of 5-HT would desensitize the 5-HT3 receptors that enhance the release of NA.

This article entitled "Effect of long-term administration of antidepressant drugs on the 5-HT3 receptors that enhance the electrically evoked release of $[3 \mathrm{H}]$ noradrenaline in the rat hippocampus" by myself, Claude de Montigny and Pierre Blier was in press in the European Journal of Pharmacology at the moment of completing this thesis. 


\title{
Effect of long-term administration of antidepressant drugs on the
}

\section{$5-\mathrm{HT}_{3}$ receptors that enhance the electrically evoked release of}

\author{
$\left[{ }^{3} \mathrm{H}\right]$ noradrenaline in the rat hippocampus
}

\author{
Raymond Mongeau, Claude de Montigny and Pierre Blier \\ Neurobiological Psychiatry Unit, Department of Psychiatry, McGill University, 1033 Pine Avenue West, Montrcal, Québec. Canada H3A IAI
}

Received 2 June 1994; revised MS reccived 2 August 1994; accepted 6 September 1994

\begin{abstract}
The present study investigated the effects of various classes of antidepressant dngs $(10 \mathrm{mg} / \mathrm{kg} / \mathrm{day}$, s.c. during 21 days) on the electrically evoked release of $\left[{ }^{3} \mathrm{H}\right]$ noradrenaline and on its modulation by the $5-\mathrm{HT}_{3}$ receptor agonist 2methyl-5-hydroxytryptamine (2-methyl-5-HT) using preloaded rat hippocampal slices. Treatments with either fluoxetine, a selective serotonin (5-HT) reuptake inhibitor, or moclobemide, a reversible type A monoamine oxidase inhibitor, increased the evoked release of $\left[{ }^{3} \mathrm{H}\right]$ noradrenaline. These two antidepressant treatments did not change, however, the magnitude of the enhancing effect of 2-methyl-5-HT on the electrically evoked release of [ $\left.{ }^{3} \mathrm{H}\right]$ noradrenaline. Desipramine produced a much larger increase of the electrically evoked release of $\left[{ }^{3} \mathrm{H}\right]$ noradrenaline than fluoxetine or moclobemide. and desensitized the 5-HT 3 receptors that modulate this release. Trimipramine, which like desipramine has a tricyclic structure but does not block the reuptake of noradrenaline or that of 5-HT, did not increase the evoked release of ${ }^{[} \mathrm{H}$ H]noradrenaline and did not desensitize the $5-\mathrm{HT}_{3}$ receptors that enhance the release of $\left[{ }^{3} \mathrm{H}\right]$ noradrenaline. Maprotiline, a selective noradrenaline reuptake inhibitor, did not produce the same changes as desipramine, but maprotiline inhibited noradrenaline reuptake to a lesser extent (50\%) than desipramine (80\%). These results suggest that the high potency noradrenaline reuptake blocker desipramine desensitizes 5 - $\mathrm{HT}_{3}$ receptors modulating $\left[{ }^{3} \mathrm{H}\right.$ ]noradrenaline release, but that this effect is not common to all antidepressant drugs.
\end{abstract}

Key words: Antidepressant drugs; Desipramine; Fluoxetine; Maprotiline; Moclobemide; Trinipramine; 2-Methyl-5hydroxytryptamine; 5-HT (5-hydroxytryptamine); Reuptake inhibition; Hippocampus; Presynaptic modulation.

\section{Introduction}

The primary effect of many antidepressant drugs is to prolong the synaptic action of noradrenalineand serotonin (5-HT) (Maître et al., 1975; Wong et al., 1975; Ross and Renyi, 1977; Blackburn et al., 1978; Hyr:el, 1982; Waldmeier et al., 1982; Thomas et al., 1987). A critical issue that needs to be resolved in order to fully understand the mechanism of action of antidepressant dnugs is whether they can alter interactions between the noradrenergic and the serotonergic systems. It was therefore deemed important to investigate the effect of long-term antidepressant drug treatments on 5-HT recepiors modulating noradrenaline release. We recently reported that $5-\mathrm{HT}, 2-$
methyl-5-HT (a 5-HT 3 receptoragonist), and 5-HT reuptake inhibition enhance the electrically evoked release of $\left[{ }^{3} \mathrm{H}\right]$ noradrenaline in the rat limbic system, effects which are blocked by $5-\mathrm{HT}_{3}$ receptor antagonists (Mongeau et al., 1994a).

It is noteworthy that the 5-HT, 3 receptor agonist mchlorophenylbiguanide was also found to enhance the electrically evoked release of $[3 \mathrm{H}]$ noradrenaline, but this effect occurs at concentrations well above the affinity of this dng for $5-\mathrm{HT}_{3}$ receptors and is not blocked by $5-\mathrm{HT}_{3}$ antagonists (Mongeauetal., 1994a: Schlickeretal., 1994). This enhancing effect of m-chlorophenylbiguanide was 
shown to be mediated by $\alpha_{2}$-adrenergic autoreceptors (Schlicker et al., 1994). Although 2-methyl-5-HT has a weak affinity for $\alpha_{2}$-adrenergic binding sites (Schlicker et al., 1994), its enhancing on the electrically evoked release of $\left[{ }^{3} \mathrm{H}\right]$ noradrenaline in the rat hippocampus does not involve $\alpha_{2}$-adrenergic autoreceptors. Indeed, 2-methyl-5HT did not block the inhibitory effect of an $\alpha_{2}$-adrenergic autoreceptor agonist and displayed its usual enhancing effect in conditions where there is no autoinhibion through $\alpha_{2}$-adrenergic autoreceptors (Mongeau et al., 1994a).

Electrophysiological studies have shown that longterm treatments with selective 5-HT reuptake inhibitors or monoamine oxidase inhibitors enhance the efficacy of the electrical stimulation of the 5-HT pathway in suppressing the firing activity of rat hippocampus pyramidal neurons (Blierandde Montigny, 1994). In contrast, treatments with monoamine oxidase inhibitors do not change the suppressing effect of the stimulation of the noradrenergic pathway on these neurons (Blier et al., 1986). Superfusion experiments carried out in the guinea-pig hippocampus have shown that long-term treatments with a monoamine oxidase inhibitor or a selective 5-HT reuptake inhibitor increase the electrically evoked release of [3H]5-HT (Blier and Bouchard, 1994). Furthermore, the increase in rat brain 5 HT concentrations is higher following long-term than following acute administration of monoamine oxidase inhibitors (Blier etal., 1986). These findings are consistent with the fact that, at the beginning of a treatment with a monoamine oxidase inhibitor, there is a decrease in the firing rate of 5-HT neurons, but upon long-term administration, the firing activity of these neurons progressively normalizes and there ensues a net increase in 5-HT neurotransmission (Blier et al., 1986). A recent microdialysis study has shown thata long-term monoamine oxidase inhibitor treatment increases to a much greater exient the release of noradrenaline in the rat frontal cortex than an acute monoamine oxidase inhibitor treatment, despite similar degrees of enzyme inhibition being achieved (Finberg et al., 1993). Contrary to the increased 5-HT neurotransmission, however, the increase in noradrenaline release following long-term monoamine oxidase inhibitors appears inconsistent with the fact that the firing activity of locus coeruleus noradrenergic neurons is steadily decreased by a long-term monoamine oxidase inhibitor treatment (Blier and de Montigny, 1985).

The changes in 5-HT release induced by long-term treatments with monoamine oxidase inhibitors and selective 5-HT reuptake inhibitors might alter secondarily the reltease of noradrenaline. However, the occurrence of such modulation would depend on the sensitivity of 5-HT receptors modulating the release of noradrenalint being unaltered by these antidepressant treatments. Recent data from our laboratory have shown that $5-\mathrm{HT}_{3}$ receptors exerting a positive feedback on 5-HT release in the guineapighippocampus desensitize during a long-term treatment a selective 5-HT reuptake inhibitor (Blier and Bouchard,
1994). It was thus deemed crucial to test whether the sensitivity of the 5- $\mathrm{HT}_{3}$ receptors that enhance the release of $\left[{ }^{3} \mathrm{H}\right]$ noradrenaline can be altered by any of the major classes of antidepressant drugs. The following drugs were studied: 1) fluoxetine as a selective 5-HT reuptake inhibitor (Wong et al., 1975); 2) moclobemide as a reversible type A monoamine oxidase inhibitor (Baldwin and Rudge. 1993); 3) desipramine as a tricyclic antidepressant drug that blocks noradrenaline reuptake (Glowinskiand Axelrod. 1964); 4) trimipramine as a tricyclic antidepressant drog devoid of noradrenaline- and 5-HT-reuptake blocking properties (Randrup and Braestrup, 1977; Hyttel, 1982); and 5) maprotiline as a selective noradrenaline reuptake inhibitor (Maitre et al., 1975). The siove drugs have been shown to have comparable antidepressant efficacy in clinical trials (Pecknold et al., 1985; Cournoyer et al., 1987; Laux et al., 1989; Bowden et al., 1993; Judd et al., 1993; Williams et al., 1993).

\section{Materials and Methods}

\section{I Treatments}

Male Sprague-Dawley rats $(175-200 \mathrm{~g})$, maintained on a 12:12 h light-dark cycle with free access to food and water, were treated for three weeks with saline or $10 \mathrm{mg} /$ $\mathrm{kg} /$ day of either fluoxetine, moclobemide, desipramine, trimipramine or maprotiline using osmotic minipumps (Alzet) implanted subcutaneously. These minipumps were removed under halothane anesthesia $48 \mathrm{~h}$ before the superfusion experiment to allow elimination of the drugs.

In the $\left[{ }^{3} \mathrm{H}\right]$ noradrenaline uptake experiments, rats $(250-275 \mathrm{~g})$ were treated for 2 days with saline or $10 \mathrm{mg} /$ $\mathrm{kg} /$ day of either desipramine or maprotiline using osmotic minipumps (Alzet) implanted subcutaneously. The minipumps containing the drugs were left in place at the time of sacrifice.

\subsection{Superfusion experiments}

Rats (350-375 g) were sacrificed by decapitation and their brain immediately removed and rapidly dissected on an ice-cold glass plate. Slices $400-\mu \mathrm{m}$ thick from the hippocampus (about $3 \mathrm{~mm}^{2}$ in surface) were prepared using a McIl wain chopper and incubated for $30 \mathrm{~min}$ at $37 \circ \mathrm{C}$ in Krebs buffer containing $0.1 \mu \mathrm{M}$ [ $\left.{ }^{3} \mathrm{H}\right]$ noradrenaline (specific activity $15 \mathrm{mCi} / \mathrm{mmol}$ ) and bubbled with a mixture of $95 \% \mathrm{O}_{2} / 5 \% \mathrm{CO}_{2}$. The composition of the Krebs buffer in mM concentrations was: $\mathrm{NaCl} 118, \mathrm{KCl} 4.7$, $\mathrm{CaCl}_{2} 1.3, \mathrm{MgCl}_{2}$ 1.2, $\mathrm{NaH}_{2} \mathrm{PO}_{4} 1, \mathrm{NaHCO}_{3} 25$, glucose 11.1. Na2EDTA 0.004 and ascorbic acid 0.11. At the end of the 30-min [ $\left.{ }^{3} \mathrm{H}\right]$ noradrenaline uptake period, the slices were washed in Krebs buffer to remove the free [ $3 \mathrm{H}]$ noradrenaline, and then three slices of the hippocampus were transferred into each glass chamber (volume $1.85 \mathrm{ml}$ ) and superfused at rate of $0.5 \mathrm{ml} / \mathrm{min}$ with oxygenated Krebs buffer maintained at $37{ }^{\circ} \mathrm{C}$. Nineteen consecutive $4-$ $\mathrm{min}$ fractions were collected starting $60 \mathrm{~min}$ after the 
beginning of superfusion. The slices were stimulated twice, at $8 \mathrm{~min}\left(\mathrm{~S}_{1}\right)$ and at $56\left(S_{2}\right)$ inin), after the end of the washing perict. The elcitrical field generated in the chambers betweer: the two platinum electrodes $(2 \mathrm{~cm}$ apart) had a voltage drop of about $5 \mathrm{~V} / \mathrm{cm}$. The folluwing stimulation parameters were used: $20 \mathrm{~mA}, 2 \mathrm{~ms}, 3 \mathrm{~Hz}$ for $2 \mathrm{~min}$. The first stimulation period $\left(S_{1}\right)$ was alwavs used as control and 2-methyl-5-HT $(10,30,100 \mu \mathrm{M})$ was added 8 $\min$ before $S_{2}$ and remained present until the end of the experiment. Nisoxetine, a selective noradrenaline reuptake inhibitor (Fuller et al., 1979), was present throughout superfusion period to avoid the displac sment of [ ${ }^{3} \mathrm{H}$ ] noradrenaline from noradrenaline terminals by 2 methyl-5-HT. At the end of the experiments, the slices wise solubilized in $0.5 \mathrm{ml}$ of Soluene 350 and the radioactivity in the slices and superfusate samples was determined by liquid scintillation spectrometry using $5 \mathrm{ml}$ of Ready Micro at $50 \%$ efficiency using a Beckman LS 1600 counter. Between 8 and 19 experiments were performed in individual superfusion chambers for each concentration of 2-methyl-5-HT and 4 to 6 animals were used to generate each concentration-effect curve. The amount of tritium released per 4-min sample was expressed as a fraction of the total tissue tritium content at the onset of the respective collection period. The overflow of tritium produced by the electrical stimulation was calculated as the total increase in radioactivity above the basal outflow of tritium determined in the sample immediately preceding the onset of stimulation. Toassess the drug-induced changes of electrically evoked release of tritium from the slices preloaded witn [ $\left.{ }^{3} \mathrm{H}\right]$ noradrenaline, $S_{2} S_{1}$ ratios were calculated, and $\mathrm{B}_{2} / \mathrm{B}_{1}$ ratios were also calculated to determine whether the drugs had altered the basal outflow of radioactivity.

\subsection{Uptake experiments}

Hippocampal slices were prepared as for superfusion experiments and pre-incubated for $3 \mathrm{~min}$ at $37{ }^{\circ} \mathrm{C}$ in Kreis buffer. After the pre-incubation period, $\left[{ }^{3} \mathrm{H}\right]$ noradrenaline was added at a final concentration of $20 \mathrm{nM}$. Following a $3 \mathrm{~min}$ incubation period, uptake was terminated by transferring the slices to $5 \mathrm{ml}$ of ice-cold buffer, and they were then solubilized in $0.5 \mathrm{ml}$ of Soluene 350. Parallel experiments were carried out at $0{ }^{\circ} \mathrm{C}$ as controls for passive diffusion. Radioactivity in the slices and in the incubation medium was determined by liquid scintillation spectroscopy. All experiments were performed 3 to 4 times and the amount of tritium actively captured by the tissue $\left(C_{A}\right)$ was calculated according to the formula $C_{A}=C_{T}-C_{P}$, where $C_{T}$ and $C_{P}$ are the tissue/ medium ratios of [ ${ }^{3} \mathrm{H}$ ]noradrenaline at $37{ }^{\circ} \mathrm{C}$ (total) and $0{ }^{\circ} \mathrm{C}$ (passive), respectively. The percentage inhibition was calculated by comparing the $\boldsymbol{C}_{\boldsymbol{A}}$ values obtained in the slices prepared from the control and treated rats.

\subsection{Statisticui analyses}

All results are expressed as means \pm S.E.M. Possible differences in mean evoked release $\left(S_{i}\right)$ between treated and control groups were tested with the two-tailed Student's $t$ test. A two-way ANOVA was used to compare the concentration-effect curves for 2-methyl-5-HT in saline and drug treated groups, concentration and ireatment being the two factors. The results of the reuptake experiments were analysed with the two-tailed Student's $t$ test. Probability $(P)$ values smaller than 0.05 were considered as significant.

\section{Drugs}

The following drugs were used: 2-methyl-5-HT and maprotiline (Sigma Chemicals, St-Louis, MO, USA), [3H]noradrenaline $\mathrm{HCl}$ (NEN, Mississauga, Ont.. Can.), fluoxetine $\mathrm{HCl}$ (Eli Lilly, Indianapolis, IN, USA), moclobemide (Hoffman-La Roche, Basel, Switzerland), desipramine (RBI, Natick, MA, USA), trimipramine bismaleate (Rhône-Poulenc, Montreal, Can.), nisoxetine (Eli Lilly, Indianapolis, Ind, USA).

\section{Resuits}

3.1 Effects of long-term fluoxetine, moclobemide and desipramine treatments

In a first series of experiments, the effect long-term inhibition of monoamine oxidase $\mathrm{A}$ with moclobemide (Baldwin and Rudge, 1993), or long-term blockade of the 5HT reuptake carrier with fluoxetine (Wong et al., 1975) using $10 \mathrm{mg} / \mathrm{kg} /$ day $\times 21$ days of either drug was assessed for their effects on the evoked release of $\left[{ }^{3} \mathrm{H}\right]$ noradrenaline and its modulation by $5-\mathrm{HT}_{3}$ receptor activation. The minipumps containing the drugs were removed $48 \mathrm{~h}$ before the experiments to allow elimination of the drugs. As shown in Fig. 1, there was a small but significant increase of the electrically evoked release of $[3 \mathrm{H}]$ noradrenaline $\left(S_{1}\right)$ from rat hippocampal slices after either the fluoxetine or moclobemide treatments. The enhancing effects of these long-term treatments were of about the same amplitude. i.e. $16 \%$ with fluoxetine and $18 \%$ with moclobemide. However, the basal outflow of radioactivity $\left(B_{1}\right)$ was not altered by these treatments (fluoxetine: $1.47 \pm 0.03 \%, \mathrm{n}=38$ vs saline: $1.44 \pm 0.03 \%, n=38$; moclobemide: $1.32 \pm 0.02 \%$, $n=47$ vs saline $=1.27 \pm 0.02 \%, n=47$ ). The amount of radioactivity recovered in slices at the end of the experiments was slightly reduced in the moclobemide group (moclobemice: $40.7 \pm 1.7 \mathrm{nC}, \mathrm{n}=47$ vs saline: $46.8 \pm 1.4$ $n C, n=47 ; P<0.01$, and similar to controls in the fluoxetine group (fluoxetine: $33.3 \pm 1.8 \mathrm{nC}, \mathrm{n}=38$ vs saline: $34.9 \pm 2.4$ $\mathrm{nC}, \mathrm{n}=38$ ).

It was previously shown that the activation of $5-\mathrm{HT}_{3}$ receptors with the agonist 2-methyl-5-HT concentrationdependently enhances the evoked release of $\left[{ }^{3} \mathrm{H}\right]$ noradrenaline from rat hippocampal slices (Mongeau et al.. 1994a). This effect was consistently reproduced in all 
the groups of this series of experiments (Fig. 2). Indeed, 2methyl.5-HT introduced in the medium $8 \mathrm{~min}$ before $S_{2}$ produced a significant concentration-dependent increase in the evoked release of $\left[{ }^{3} \mathrm{H}\right]$ noradrenaline without altering the basal efflux of radioactivity. The mean percentage increases in the $S_{2} / S_{1}$ ratios produced by 10,30 and $100 \mu \mathrm{M}$ of 2-methyl-5-HT in the saline groups of this series of experiments were, respectively, $15 \%, 30 \%$ and $47 \%$. Longterm treatment with either a drug that prolongs the synaptic action of 5-HT, fluoxetine, or with one that increases the neuronal pool of 5-HT, moclobemide, did not alter the magnitude of the effect of activating 5-HT3 receptors on the evoked release of [ $\left.{ }^{3} \mathrm{H}\right]$ noradrenaline. Indeed, the potency of the 5-HT3 receptor agonist in the fluoxetine and the moclobemide groups was similar to that in control rats nun in parallel (Fig. 2A, 2B).

In contrast, a long-term treatment with desipramine ( $10 \mathrm{mg} / \mathrm{kg} /$ day $\times 21$ days), a noradrenaline reuptake inhibitor(Glowinskiand Axelrod, 1964), considerably decreased the effect of 30 and $100 \mu \mathrm{M}$ of 2-methyl-5-HT to enhance the evoked release $\left[{ }^{3} \mathrm{H}\right]$ noradrenaline (Fig. $2 \mathrm{C}$ ). At these

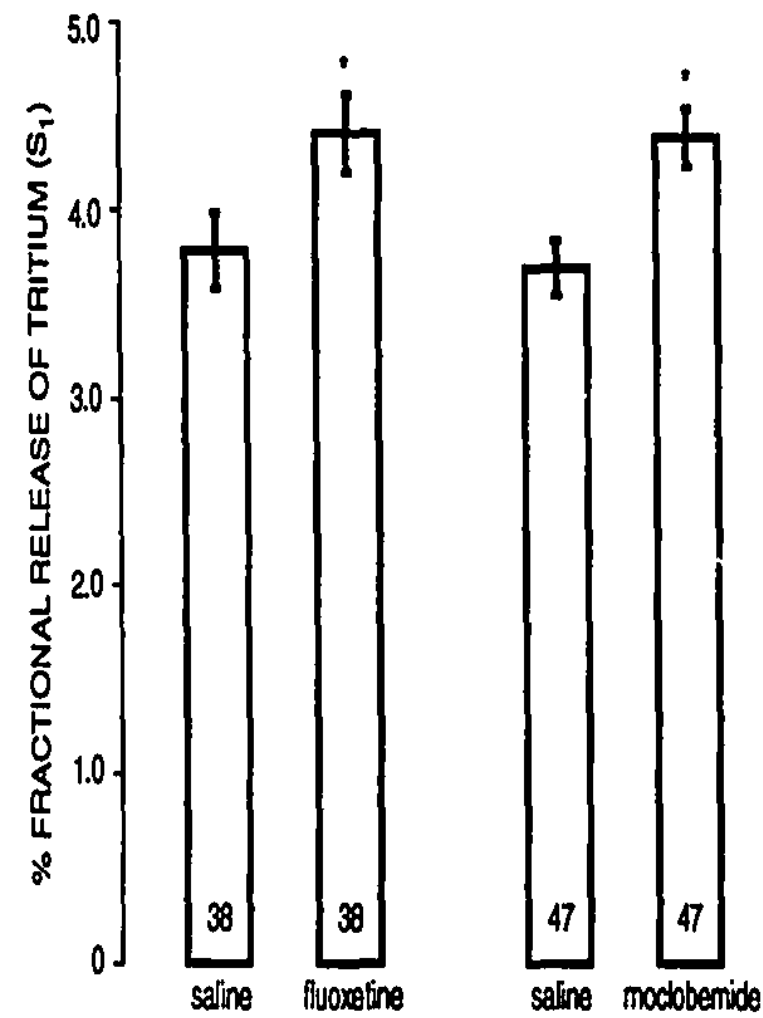

Fig. 1. Effect of long term treatments with $10 \mathrm{mg} / \mathrm{kg} / \mathrm{day} \times 21$ days of either fluoxetio or moclobemide on the relesse of tritiun elicitod by the oloctrical stimulation of hippocampal slices preloaded with [3H]noradreralino auporfused with $\&$ Krebs buffor contrining $1 \mu \mathrm{M}$ of nisoxetine. Ordinute depicts the percentage of radiosctivity relensed by 360 pulses of $2 \mathrm{~ms}$ delivered at an imentity of $20 \mathrm{~mA}$ and a froquency of $3 \mathrm{~Hz}$ Dath aro expressed as mean \pm S.E.M.M. of the number of experiments indicated inside the columns. $P<0.05$, using the two-tsiled 5udent'st tox.

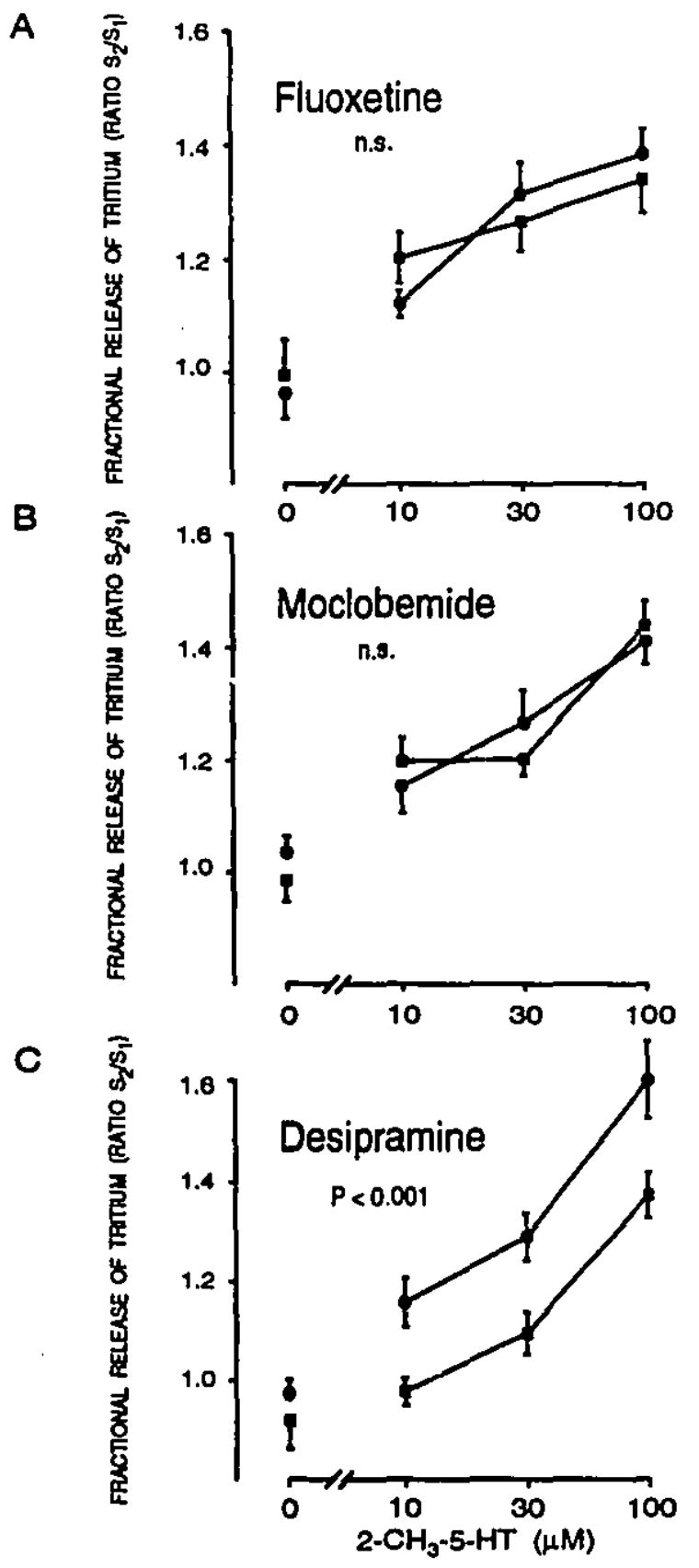

Fig. 2. Concentration-effect curves of 2-methyl-5-HT, introduced $8 \mathrm{~min}$ before $S_{2}$ in a Kreba buffer containing $1 \mu$ M of nisoxetine, on the release of tritium elicited by the electrical stimulution of hippocampal slices prepared from rats treated with aline or $10 \mathrm{mg} / \mathrm{kg} / \mathrm{day} \times 21$ days of either fluoxetine (A), moclobemido (B) or destpremino (C). Tho circle and square symbols represent experiments with allino-trealod and drug-treated rats, respectively. Ontinate depicts the fruction of toul tissue radionctivity released by 360 polses $\left(20 \mathrm{~mA}, 2 \mathrm{~ms}, 3 \mathrm{~Hz}\right.$ ) expresed as the ratio $\left(\mathrm{S}_{2} \mathrm{~S}_{1}\right)$ obtained between the recond period of atimulation in the presence of 2-methyl-5-HT (S2) and tho firs one done withou this drug (S1). Each point represents the mean \pm S.E.M of the number of experiments which were of 8-10 (A), 8.14 (B) and 9-19 (C). Two-way ANOVA revealed a significanl effoct $(P<$ $0.001)$ for concestration for all the groups and a significant effect $(P<$ 0.001) for the desipromino treutmen. 
two concentrations, there was respectively $44 \%$ and $29 \%$ less enhancement in the desipramine group compared to thi matched control group. Furthermore, $10 \mu \mathrm{M} 2$-methyl-5HT did not produce any significant effect in slices from rats treated with desipramine(Fig. 2C). This decreased potency of 2-methyl-5-HT in the desipramine group was accompanied with a large increase $(77 \%)$ in the evoked release of [ ${ }^{3} \mathrm{H}$ ]noradrenaline in $\mathrm{S}_{1}$ (Fig. 3). However, this treatment did not change the spontaneous outflow of radioactivity (desipramine: $1.14 \pm 0.03 \%, n=57$; saline: $1.16 \pm 0.03 \%$. $\mathrm{n}=57$ ), but decreased the amount of radioactivity recovered in the slices at the end of the experiments (desipramine: 42.8 $\pm 1.8 \mathrm{nC}, \mathrm{n}=57$ vs saline: $51.3 \pm 1.3 \mathrm{nC}, \mathrm{n}=57 ; \mathrm{P}<0.001$ ).

Before concluding an attenuated sensitivity of the 5 . $\mathrm{HT}_{3}$ receptors, it was considered essential to determine whether such a large increase in the evoked release of $[3 \mathrm{H}]$ noradrenaline could interfere with a reliable analysis of the effect of 2-methyl-5-HT by prolonging the time during

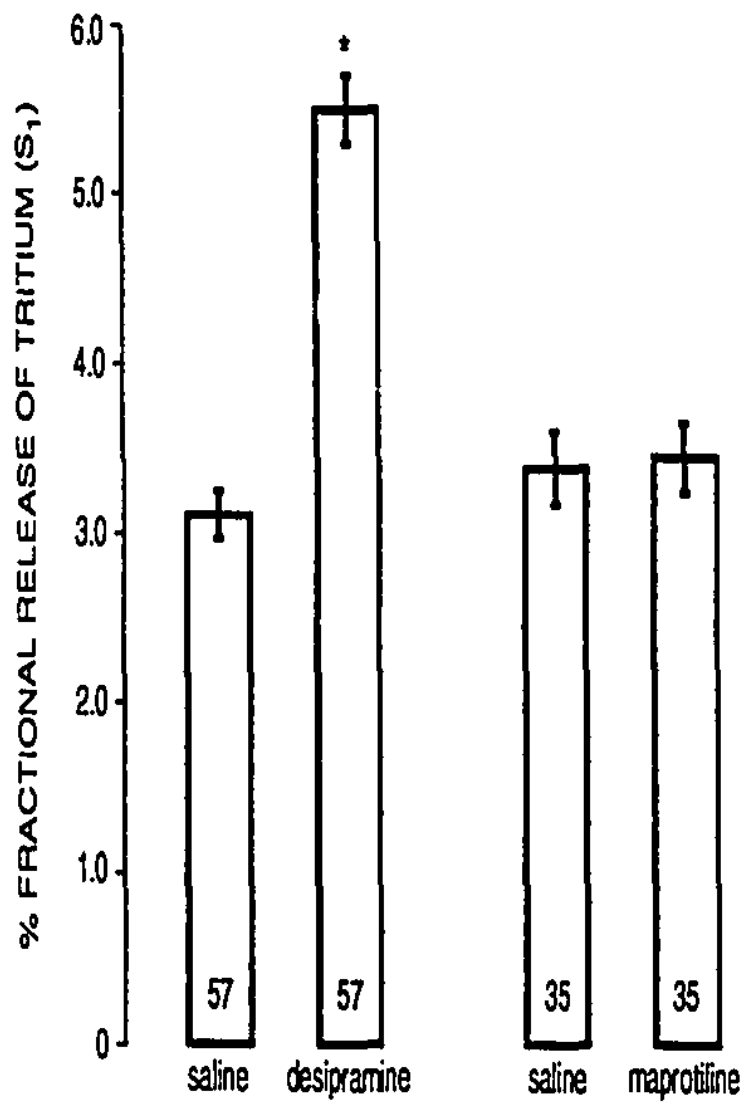

Fig. 3. Effect of long-tem treatments with $10 \mathrm{mg} / \mathrm{kg} / \mathrm{day} \times 21$ days of cither desipramine ormaprotiline on the release of tritium elicitod by the eloctrical stimulation of hippocampal slices prelosded with [3 H] nondrenalino supertused with a Krebs buffer contuining I $\mu \mathrm{M}$ nisoxetine. Ondinato depicts the percentage of ndiostivity relessed by 360 pulses $(20 \mathrm{~mA}, 2 \mathrm{~ms}$, $3 \mathrm{~Hz}$ ). Duta are expressed as mean \pm S.E.M of the number of experiments isdiculed inside the columns. $\bullet P<0.001$, using the two-tailed Student's t test. which the release process occurs. Considering that the overflow of $\left[{ }^{3} \mathrm{H}\right]$ noradrenaline is generally enhanced not only in amplitude but also in duration by 2-methyl-5-HT, it was possible therefore that a large increase in the evoked release would have caused an underestimation of the enhancing effect of the 5- $\mathrm{HT}_{3}$ receptor agonist in the desipramine group. Further experiments in desipramine treated-rats were thus carried out and the overflow was collected and analysed for 30 instead of $22 \mathrm{~min}$ after the first and the second stimulation period. The $S_{2} / S_{1}$ values obtained were identical to those obtained with a $22 \mathrm{~min}$ collection (data not shown), indicating that it is not necessary to take into account the overflow of tritium sometimes present after the end of the 22 -min period to estimate reliably the $S_{2} / S_{1}$ values. More importantly, the decreased potency of 2-methyl-5-HT after the desipramine treatment was still observed in this condition. For example, when the analysis of the overflow was carried out over a 22-min period, $30 \mu \mathrm{M}$ of 2 -methyl-5-HT produced a $46 \%$ increase in the saline group and a $33 \%$ increase in the desipramine group, and analysing the overflow over a 30 -min period indicated similar increases, i.e. $49 \%$ and $34 \%$ respectively.

\subsection{Comparison of the effects oflong-term trimipramine and maprotiline treatments with that of desipramine}

Other long-term antidepressant treatments were studied to determine whether antidepressant drugs sharing with desipramine either a tricyclic structure or the property of blocking noradrenaline reuptake would also desensitize the $5-\mathrm{HT}_{3}$ receptors that modulate $\left[{ }^{3} \mathrm{H}\right]$ noradrenaline release. First, trimipramine $(10 \mathrm{mg} / \mathrm{kg} /$ day $\times 21$ days) was studied because this tricyclic antidepressant drug blocks neither 5 HT nor noradrenaline reuptake (Randrup and Bracstrup, 1977; Hyttel, 1982). Second, the antidepressant maprotiline ( $10 \mathrm{mg} / \mathrm{kg} /$ day $\times 21$ days) was also studied since it is a tetracyclic which selectively blocks noradrenaline reuptake (Maitre et al., 1975).

Neither the trimipramine nor the maprotiline treatment produced any changes in the evoked release or spontaneous outflow of $\left[{ }^{3} \mathrm{H}\right]$ noradrenaline (trimipramine: $\mathrm{S}_{1}=$ $3.73 \pm 0.20, B_{1}=1.35 \pm 0.03 \%, n=34$ vs saline: $S_{1}=3.86$ $\pm 0.21, B_{1}=1.32 \pm 0.04 \%, n=36$; maprotiline: $S_{1}$ see Fig. $3, B_{1}=1.09 \pm 0.03 \%, n=35$ vs saline: $B_{1}=1.05 \pm 0.03 \%$. $\mathrm{n}=35$ ). The amount of adioactivity recovered in the slices of these two groups were also similar to their respective controls (trimipramine: $43.9 \pm 2.0 \mathrm{nC}, \mathrm{n}=34$ vs saline: 43.9 $\pm 1.8 \mathrm{nC}, \mathrm{n}=36$; maprotiline: $55.2 \pm 2.4 \mathrm{nC}, \mathrm{n}=35$ vssaline: $55.8 \pm 2.2 \mathrm{nC}, \mathrm{n}=35$ ). The mean percentage increase in the $\mathrm{S}_{2} / \mathrm{S}_{1}$ ratios produced by 10,30 and $100 \mu \mathrm{M}$ of 2 -methyl-5HT in the saline groups was of $20 \%, 31 \%$ and $71 \%$, respectively. Neither trimipramine nor maprotiline altered the effect of 2-methyl-5-HT on the evoked relense of [3H]noradrenaline. The concentration-effect curves of 2 methyl-5-HT in the trimipramine and maprotiline groups were not different from those of their matched controls (Fig.4A and 4B). 
Maprotiline was compared to desipramine for its efficacy in blocking $\left[{ }^{3} \mathrm{H}\right]$ noradrenaline uptake. Two groups of rats received $10 \mathrm{mg} / \mathrm{kg} /$ day for 2 days of either drug while a third group received saline for the same period. The minipumps containing the drugs were kept in the animals at the time of sacrifice. As expected, both drug treatments reduced, when compared to the saline group, the uptake of $\left[{ }^{3} \mathrm{H}\right]$ noradrenaline in hippocampus slices (tissue / medium ratios of tritium; desipramine: $0.33 \pm 0.10 . n=5$; maprotiline: $0.83 \pm 0.18, n=5$; saline: $1.57 \pm 0.13, n=5$ ). However, the degree of inhibition of $\left[{ }^{3 \mathrm{H}}\right]$ noradrenaline uptake by the desipramine treatment $(80 \%)$ was significantly greater $(\mathrm{P}<$ 0.05 ) than that produced by the maprotiline treatment $(50 \%)$.

A

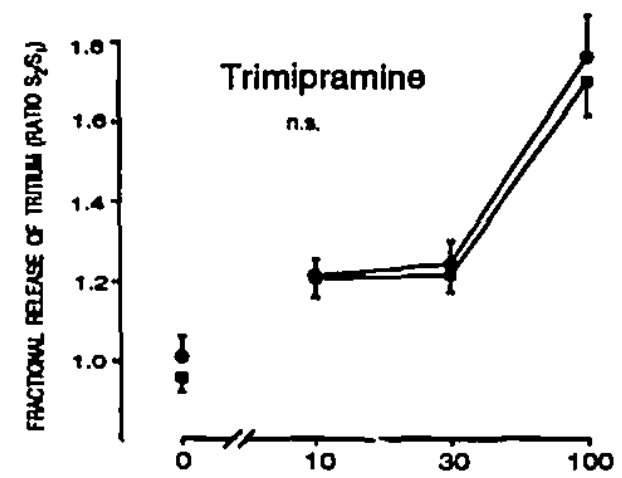

B

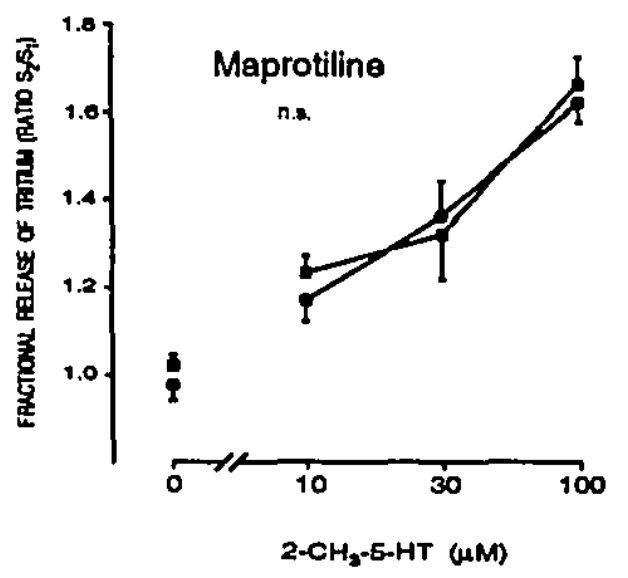

Fig. 4. Concentration-effoct curves of 2-methyl-5-HT, introduced $8 \mathrm{~min}$ before $S_{2}$ in a Krebs buffer contenining $1 \mu \mathrm{M}$ of nisoxetine, on the release of tritium elicited by the electrical stimulation of hippocampal slices prepared from rats treated withsaline or $10 \mathrm{mg} / \mathrm{kg} / \mathrm{day} \times 21$ days of either trimipounine (A) or maprotiline (B). The circle and square symbols represent experiments with saline-treated and drug-treseted rits, respectively. Ordinne lepicts the fraction of total tissue radionctivity released by 360 pulses (20 $\mathrm{mA}, 2 \mathrm{~ms}, 3 \mathrm{~Hz}$ ) expressed as the ratio ( $\mathrm{S}_{2} \mathrm{~S}_{1}$ ) obtuinod between the second period of stimulation in the presenco of 2-methyl-5-HT(S2) and the fint ono done without this drug $\left(S_{1}\right)$. Each point represents the mean $\pm S E . M$ of tho number of experiments which were of 8-10 (A) and \&-10 (B). Two-way ANOVA revealed a significant effect for concentration $(P<0.001)$ for all the groups without any significant effect for treament.

\section{Discussion}

Fluoxetine prolongs the synaptic action of 5-HT and moclobemide increases the neuronal pool of 5-HT via the inhibition of reuptake and monoamine oxidase, respectively (Wong et al., 1975; Baldwin and Rudge, 1993). Longterm treatment with these drugs did not change the sensitivity of the $5-\mathrm{H}_{13}$ receptors that enhance the release of $\left[{ }^{3} \mathrm{H}\right]$ noradrenaline in the rat hippocampus (Fig. 2). It is interesting to compare these results with those of previous studies which examined the interaction between the noradrenergic and ihe 5-HT systems mediated through $\alpha_{2-}$ adrenergic heteroreceptors of 5-HT terminals. Endogenous noradrenaline tonically inhibits the release of 5-HT by activating these heteroreceptors in the rat hippocampus (Mongeau et al., 1993). Long-term inhibition of noradrenaline re uptake or monoamine oxidase desensitize these $\alpha_{2}$-adrenergic heteroreceptors, an effect that was shown to result from a sustained increase in noradrenaline synaptic concentration (Mongeau et al., 1994b). Contrary to $\alpha_{2}$-adrenergic heteroreceptors, the $5-\mathrm{HT}_{3}$ receptor that enhances the release of [ ${ }^{3 H}$ ] noradrenaline does not appear to be tonically activated by its endogenous neurotransmitter under basal conditions. Two observations support this contention: 1) 5-HT 3 receptor antagonists do not decrease the evoked release of $\left[{ }^{3} \mathrm{H}\right]$ noradrenaline; and, 2) 5-HT denervation does not increase the sensitivity of the $5-\mathrm{HT}_{3}$ receptors modulating [ ${ }^{3} \mathrm{H}$ ]noradrenaline release (Mongeau et al., 1994a). Differences in levels of neurotransmitters normally present in the biophase of the presynaptic fibers might account for the fact that $\alpha_{2}$-adrenergic heteroreceptors modulating 5-HT release, but not 5-HT3 receptors modulating noradrenaline release, desensitize following sustained increase in the concentration of neurotransmitter acting at these receptors.

Long-term treatments with moclobemide increased the evoked release of $\left[{ }^{3} \mathrm{H}\right]$ noradrenaline from rat hippocampal slices (Fig. 1). This observation cannot be explained by an inhibition of monoamine oxidase at the time of the experiment since moclobemide is a reversible monoamine oxidase inhibitor and the minipump containing the drug had been removed $48 \mathrm{~h}$ before the experiment. This result is consistent with those of a recentmicrodialysis study which showed that long-term inhibition of monoamine oxidase A by clorgyline increases the release of noradrenaline in the rat frontal cortex more than an acute treatment with this dru, (Finherg etal., 1993). This increase in noradrenaline release could not readily be explained by differences in the degree of monoamine oxidase inhibition as there was near maximal inhibition of the enzyme after both acute and longterm treatments (Finberg et al., 1993). Long-term changes in the basic mechanisms of noradrenaline release have to be considered to explain the enhancing effect of moclobemide. The prolonged increase in the synaptic concentration of noradrenaline induced by moclobemide might have decreased the sensitivity of terminal $\alpha_{2}$-adrenergic autoreceptors and consequently increased the evoked re- 
lease of $\left[{ }^{3} \mathrm{H}\right]$ noradrenaline. Toourknowledge, no superfusion studies have yet directly assessed the effect of long-term moclobemide on the sensitivity of terminal $\alpha_{2}$-adrenergic autoreceptors. Nevertheless, studies have shown that longterm treatments with monoamine oxidase inhibitors increase the release of $\left[{ }^{3} \mathrm{H}\right]$ noradrenaline but do not change the sensitivity of the $\alpha_{2}$-adrenergic autoreceptors in the hippocampus (Campbell and McKeman, 1986; Palfreyman et al., 1986; Blier and Bouchard, 1994). There is also the possibility that more storage vesicles are released per action potential during long-term moclobemide treatment. However, this appears unlikely considering that the levels of dopamine $\beta$-hydroxylase, an enzyme co-released with noradrenaline, is normally reduced after long-term administration of monoamine oxidase inhibitors (Finberg et al., 1993). An alternative hypothesis is that long-term changes in 5-HT release induced by monoamine oxidase inhibitors might have secondarily altered the evoked release of [3H]noradrenaline. This possibility is suggested by the fact that fluoxetine has produced a similar increase in the evoked release of $[3 \mathrm{H}]$ noradrenaline (Fig. 1). Yet, this inhibitor is highly selective for 5-HT reuptake. A recent study has shown that long-term monoamine oxidase inhibitor or selective 5-HT reuptake inhibitor treatments increase the evoked release of [ $\left.{ }^{3} \mathrm{H}\right] 5-\mathrm{HT}$ in the guinea pig hippocampus (Blier and Bouchard, 1994). It is noteworthy that these effects were attributable to alterations of the sensitivity of presynaptic receptors on 5-HT terminals rather than to the acute actions of these drugs since the minipumps containing the drugs were removed $48 \mathrm{~h}$ before the experiments to allow elimination of the inhibitors (Blier and Bouchard, 1994). Furthermore, previous electrophysiological studics in the rat hippocampus had documented an enhanced efficaiy of the electrical stimulation of the 5-HT pathway following long-term, but not acute, treatments with various selective 5-HT reuptake inhibitors and monoamine oxidase inhibitors (Blier and de Montigny, 1994). Such increases in the release of 5-HT induced by moclobemide and fluoxetine treatments might have concomitantly enhanced the release of [ $\left.{ }^{3} \mathrm{H}\right]$ noradrenaline through the activation of 5-HT receptors, such as the 5- $\mathrm{HT}_{3}$ receptors that enhance the release of $\left[{ }^{3} \mathrm{H}\right]$ noradrenaline.

A surprising finding was the reduced sensitivity of 5 $\mathrm{HT}_{3}$ receptors modulating $[3 \mathrm{H}]$ noradrenaline release following the desipramine treatment (Fig. 2). The noradrenaline reuptake blocker potency or the tricyclic structure of desipramine might account for this effect. An action of tricyclic antidepressant drugs at the 5- $\mathrm{HT}_{3}$ receptors appeared plausible considering that this class of drugs is commonly known to down-regulate, for instance, 5- $\mathrm{HT}_{2}$ receptors in binding studies (Willner, 1985). We have thus decided to test the effects of a long-term treatment with trimipramine, a tricyclic antidepressant drug that does not block the reuptake of noradrenaline (Randrup and Braestrup, 1977; Hyttel, 1982). This treatment did not change the potency of 2-methyl-5-HT, indicating therefore that desipramine was unlikely to have produced its effect on 5 $\mathrm{HT}_{3}$ receptors by a mechanism common to tricyclic antidepressant drugs. We then considered the possibility that a desensitization of $5-\mathrm{HT}_{3}$ receptors which enhance the release of $[3 \mathrm{H}]$ noradrenaline could be produced by other antidepressant drugs that block the noradrenaline reuptake carrier. Long-term treatments with maprotiline, a selective noradrenaline reuptake inhibitor (Maitre et al., 1975), did not change the sensitivity of $5-\mathrm{HT}_{3}$ receptors modulating $\left.{ }^{3} \mathrm{H}\right]$ noradrenaline release. Preliminary data have further shown that long-term treatments with nomifensine, another antidepressant drug that blocks noradrenaline reuptake, did not alter the sensitivity of these receptors (data not shown). Taken together, the above results suggest that the desensitization of the 5-HT3 receptors that enhance the release of $[3 \mathrm{H}]$ noradrenaline might not be a general property of antidepressant drugs that inhibit the reuptake of noradrenaline.

However, it cannot be excluded that the effect of desipramine on these $5-\mathrm{HT}_{3}$ receptors involved the noradrenaline reuptake carrier. Indeed, desipramine produced a greater inhibition of noradrenaline reuptake tian did maprotiline (section 3.2). This result was consistent with previous studies showing that desipramine has a greater affinity for the NA reuptake carrier than maprotiline $\left(K_{m}\right.$ values of $0.97 \mathrm{nM}$ for desipramine versus $8.4 \mathrm{nM}$ for maprotiline; Hyttel, 1982). There was also a large increase in the evoked release of $\left[{ }^{3} \mathrm{H}\right]$ noradrenaline following the desipramine treatment that was not present after the maprotiline treatment (Fig. 3). The latter effect of desipramine might be linked with a threshold level of noradrenaline reuptake blockade not having been reached with maprotiline treatment in our experimental conditions. A high degree of noradrenaline reuptake inhibition could desensitize for example, either the reuptake sites themselves or the terminal $\alpha_{2}$-adrenergic autoreceptors and consequently lead to an enhancement of the evoked release of [ $\left.{ }^{3} \mathrm{H}\right]$ noradrenaline. Indeed, there are autoradiographic data showing that noradrenaline reuptake sites are downregulated in the rat hippocampus following a long-term treatment with desipramine (Bauer and Tejani-Butt, 1992). Nevertheless, this down-regulation is not likely to be responsible for the large enhancement in the evoked release of ${ }^{3} \mathrm{H}$ ]noradrenaline since nisoxetine (a selective noradrenaline reuptake inhibitor) was present in the superfusion medium throughout experiments and should have masked the effect of a desensitization of the reuptake carrier. Consistent with the present data, other studies have found an increase in the electrically evoked release of $\left[{ }^{3} \mathrm{H}\right]$ noradrenaline in the rat brain after long-term desipramine treatment (Schoffelmeer and Mulder, 1982; Campbell and McKeman, 1986). However, no changes were generally found in the sensitivity of the $\alpha_{2}$-adrenergic autoreceptor to the inhibitory action of exogenously applied noradrenaline or clonidine (Schoffelmeer and Mulder, 1982; Campbell and McKernan, 1986; Hollingsworth and Smith, 1991). 
The decrease in the sensitivity of the 5- $\mathrm{HT}_{3}$ receptors that enhance the release of noradrenaline associated with the large increase in the release of this neurotransmitter might be the consequence of an increased intraneuronal concentration of calcium resulting from the desipramine treatment. The facts that the exocytotic release of noradrenaline has been postulated to involve intraneuronal calcium (Jackisch et al., 1992) and that the sensitivity of terminal 5-HT autoreceptors is reduced by high calcium concentration (Starke et al., 1989), support this contention. It might be argued that the desensitization of 5- $\mathrm{HT}_{3}$ receptors is a physiological mechanism that compensates for the large increase in the basal release of noradrenaline induced by the desipramine treatment. The efficacy of 5-HT in inducing an increase in the neuronal depolarization evoked release of noradrenaline would thereby be reduced by such a homeostatic mechanism. It would be of interest to know whether other drug treatments, such as amphetamine, that produce large increases in noradrenaline release could alter as well the sensitivity of the $5-\mathrm{HT}_{3}$ receptors modulating noradrenaline.

These results suggest that the high potency noradrenaline reuptakeblocker desipramine desensitizes 5$\mathrm{HT}_{3}$ receptors modulating [ $\left.{ }^{3} \mathrm{H}\right]$ noradrenaline release; however, this effect is not common to all antidepressant drugs. In most cases, enhancement in the release of 5-HT induced by antidepressant drugs could also change noradrenaline release through normosensitive $5-\mathrm{HT}_{3}$ receptors.

\section{Acknowledgement}

This work was supported in part by the Medical Research Council of Canada (MRC) grants MT-6144 and 11014 and the Fonds de la recherche en sante du Quebyri. K.M. is in receipt of a Studentship and P.B. of a Schularship from : MRC.

\section{References}

Baldwin, D. and S. Rudge, 1993, Moclobemide: a reverniblo inhibitor of monoamino oxidase type A, Br. J. Hosp. Med. 49, 497.

Baucr, M.E. and S.M. Tojani-Buth, 1992, Effects of repeated administration of desipramine or electroconvulsive shoct on norepinephrine uptato sites measured by $[3 \mathrm{H}]$ nisoxetine autoradiography, Brain Res. 582, 208.

Blackburn, T.P., G.A. Foster, D.T. Greenwood and R. Howe, 1978, Effects of viloxazine, its optical isomers and its major metabolites on bjogenic amine uptake mechanisms in vitro and in vivo, Eur. J. Pharmacol. 52, 367.

Blier, P. and C. Bouchard, 1994, Modulation of 5-HT relesse in tho guinea pig brain following long-tem administration of antidepressant druga, Br. J. Pharmacol. 113, 485..
Blier, P. and C. de Montigny, 1985, Serotonergic but not nomdrenergic neurons in rat central nervous system adapt to long-term treatment with monoamine oxjdase inhibitors, Neuroscience 16, 949.

Blier,P.,C. de Montigny and A. Azmaro, 1986, Modification of scrotonergic and noradrenergic neurotransmissions by repeated administration of monoamine oxidase inhibitors: Electrophysiological studies in the rat central nervous system, J. Phamacol. Exp. Ther. 237, 987.

Blier, P. and C. de Montigny, 1994, Current advances and trends in the treatment of depression. Trends Pharmacol. Sci. 15, 220

Bowden, C.L.,A.F.Schatzberg, A.Rosenbaum,S.A. Contreras.J.A.Samson, E. Dessain and M. Sayler, 1993, Fluoxetine and desipramine in major depressive disorder, J. Clin. Psychophamacol. 13, 305.

Campbell, I.C. and R.M. McKeman, 1986, Clorgyline and desipramine alter the sensitivity of $[3 H]$ noradrenaline relense to calcjum but not to clonidine, Brain Res. 372, 253.

Coumoyer, G., C. de Montigay, J. Ouellette, R Langlois, R. Elic. G. Caille and P. le Morvan, 1987. A comparative double-blind controlled study of trimipramine and amitriptyline in major depression: lack of correlation with 5-hydroxytryptamino reupuke blockade, J.Clin. Psychopharmacol. 7, 385.

Finberg, J.P., K. Pacak, I.J. Kopin and D.S. Goldstein, 1993, Chronic inhibition of monoamine oxidese type $A$ increases noradrenaline release in rat frontal cortex, Naunyn-Schmiedeberg's Arch. Pharmacol. 347, 500.

Fuller, R.W., H.D. Snoddy and K.W. Perry, 1979, Nisoxetine antagonism of norepinephrine depletion in brin and heart after alpha-methyl-m-tyrosine administration, Neuropharmacology 18, 767.

Glowinski, J. and J. Axelrod, 1964, Inhibition of upake of Iritiated noradrenaline in the intact rat brain by imipramine and structurally-selated compounds, Nature 204, 1318.

Hollingsworth, P.J. and C.B. Smith, 1991, Factors influencing the function

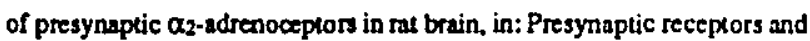
neuronal transporters, eda. S.Z. Langer, A.M. Galzin and J. Costentin (Pergemon, Oxford) p. 39.

Hytsel,J., 1982, Citalopram - Pharmucological profite of a specific serotonin uptake inhibitor with antidepreasant activity, Prog. Neuropsychopharmacol. Biol. Psychiatry 6. 277.

Jackisch, R, H.Y. Huang, H. Rensing, D. Lauth, C. Altgaier and G. Henting. 1992, a. -Adrenoceptor medialod inhibition of exocytotic noradrenaline release in the absence of extracellular Calcium, Eur. J. Pharmacol. Mol. Pharmacol. 226, 245.

Judd, F.K., K. Moore, T.R. Noman, G.D. Burrows, R.K. Gupta and G. Parker, 1993, A multicentre double blind trial of fluoxetine versus amitriptyline in the treatment of depressive illness, Austr. New Zealand J. Psychiatry 27. 49. 
Laux, G.,H. Beckmann, W. Classen and T.Becker, 1989,Moclobemide and maprotiline in the treatment of inpatients with major depressive disorder, J. Neural Trans. , suppl. 28,45.

Maitre, L., P.C. Waldmejer, P.M. Greengrass, J. Jaekel, S. Sedlacek and A. Delini-Stula, 1975, Maprotiline-Its position as an antidepressant in the light of recent neurophamacological and neurobiochemical findings, J. Int. Med. Res. 3, 1.

Mongeau, R., P. Blier and C. de Montigny, 1993, Invivo electrophysiological evidence for tonic activation by endogenous noradrenaline of $\alpha_{2}$ adrenoceptors on 5-hydroxytryptamine terminals in the rat hippocampus, Naunyn-Schmiedeberg's Arch. Pharmacol. 347. 266.

Mongeas, R., C. de Montigny and P. Blier, 1994a, Activation of 5-HT3 receptors enhances the electrically evoked release of [HH]noradrenaline in rit brain limbic structures, Eur. J. Pharmacol. 256, 269.

Mongeau, R., C. de Montigny and P. Blier, 1994b, Electrophysiological evidence for the desensitization of $\alpha_{2}$-adrenoceptors on serotonin terminals following long-term treatment with drugs increasing norepinephrine synnptic concentration, Neuropsychophamacology 10,41 .

Palfreyman, M.G. A.K. Mir. M. Kubinn, D.N. Middlemiss, M. Richards, M.D. Tricklebank and J.R. Fozard, 1986, Monosmine receptor sensitivity changes following chronic administration of MDL 72394, a site-directed inhibitor of monosmine oxidase, Eur. J. Phamacol. 130. 73.

Pecknold, J.C., P. Familamiri, D.J. McCluro, R. Elie and H. Chang, 1985. Trimipramine and maprotiline: antidepressant, anxiolytic, and cardiotoxic comparison. J. Clin. Psychiatry 46, 166.

Randrup. A. and C. Bracstrup, 1977. Uptake inhibition of biogenic amines by newer antidepressane drugs: relevance to the dopamine hypothesis of depression, Psychopharmacology 53, 309.

Ross, S.B. and A.L. Renyi, 1977, Inhibition of the neuronal upeske of 5 . hydroxytryptamine and noradrenaline in rat brain by (Z)- and (E).3-(4bromophenyl)-N,N-dimethyl-3-(3-pyridyl)allylamines and their seoridary analogues, Neurophamacology $16,57$.

Schlicker, E., M. Kathmann, H.J. Exner, M. Detzner and M. Gothert, 1994. The 5-HT3 receptor agonist 1-(m-chlorophenyl)-biguanide facilitates noradrenaline release by blockade of $\alpha_{2}$-adrenoceptors in the mouse brain cortex, Naunyn-Schmiedeberg's Arch. Pharmacol. 349, 20.

Schoffeimeer, A.N.M. and A.H. Mulder, 1982, ${ }^{3} \mathrm{H}$-Noradrenaline and ${ }^{3} \mathrm{H}$ 5-HT release from rat brain stices and its presynaptic $\alpha$-edrenergic modutation after long-term desipramine pretreatment. Numyn-Schmiedeberg's Arch. Pharmacol. 318, 173.

Starke, K., M. Gothert and H. Kilbinger, 1989, Moctulation of neurotransmituer release by presynaptic autoreceptors, Physiol. Rev, 69, 864.
Thomas, D.R., D.R. Nelson and A.M. Johnson. 1987, Biochemical effects of the antidepressant paroxetine, a specific 5-hydroxytrypxamine uptake inhibitor, Psychopharmacology. 93, 193.

Waldmeicr. P., P.A. Baumann, K. Hauser, L. Maitre and A. Stroni, 1982, Oxaprotiline, a norad renaline uptake inhibitor with an active and inactive enantiomer, Biochem. Phamacol. 2169.

Williams, R. R.A. Edwards, G.M. Newburn, R. Mullen. D.B. Menkes and C. Segkar, 1993, A double-blind comparison of moclobernide and fluoxetine in 'be treatment of depressive disorders, Int.Clin. Psychopharmacol. 7, 155.

Wiltiner, P., 1985. Antidepressants and serotonin, in: Depression: A Psychobiological synthesis. Wiley, New York, p. 327.

Wong, D.T., F.P. Bymater, J.S. Homg and B.B. Molloy, 1975, A new selective inhibitor for uptake of serotonin inlo synaptosomes of rat brain: 3 (p-trifuoromethylphenoxy)-N-methyl-3-phenylpropylamise.J. Pharmacol. Exp. Ther. 193, 804 . 


\section{General discussion}

The data of the present thesis provide new elements for the comprehension of the physiology of NA and 5-HT systems in limbic brain areas such as the hippocampus. Rather than being functionally isolated, these systems were shown to interact extensively through selective adrenergic and 5-HT receptors. The first study revealed that the release of 5-HT is inhibited in vivo by $\alpha_{2}$-adrenergic heteroreceptors located on 5-HT terminals. Evidence was provided indicating that these receptors are tonically activated by endogenous NA in basal cc 'itions. Moreover, the fourth study suggested that the release of NA is facilitated by the activation of 5 HT3 receptors. These receptors were shown not to be localized on 5-HT fibers. In fact, some preliminary data suggest that these 5-HT3 receptors could be heteroreceptors localized on NA fibers because the enhancing effect of 2-methyl-5-HT was observed in hippocampal slices in which the propagation of action potentials was blocked with tetrodotoxin (data not shown), as well as in hippocampal pinched-off nerve terminals (P.J. Monroe, personal communication). It also important to emphasize that these 5-HT3 receptors did not appear to be tonically activated by endogenous 5 -HT in basal conditions, albeit they were activated when the concentration of this neurotransmitter was raised by blocking the 5-HT reuptake carrier.

The presence of an inhibitory feedback mechanism involving terminal 5-HT $1 \mathrm{~B}$ or 5-HT $1 \mathrm{D}$ autoreceptors has been well documented (section 5.1). In some rare conditions, such as when depolarization is elicited with a single pulse, there is no autoinhibition of 5-HT release via these autoreceptors $^{18}$. However, in most cases this negative feedback is operative even at low frequencies of nerve depolarization, or low synaptic concentraticas of 5-HT, and thus exerts a precise and continuous control over the amount 5-HT released. The existence of an alternative feedback mechanism might be put forward on the basis of the results of the present thesis (fig. 1). An increased NA output would be triggered by $5-\mathrm{HT}_{3}$ heteroreceptors when the amount of 5-HT released from varicosities gets high enough to increase the concentration of this neurotransmitter in the biophase of noradrenergic fibers. This would in turn enhance the tonic inhibitory input of NA at $\alpha_{2}$-adrenergic heteroreceptors and, thereby, provide a negative feedback on the release of 5-HT (fig. 1).

The existence of such a regulatory loop remains only hypothetical. Further experiments would be required to determine if some aspects of 5-HT autoinhibition are impaired by inactivating NA neurotransmission. Knowing that 5-HT autoreceptor antagonists increase the evoked release of [3H]5-HT (in the presence of a SSRI) ${ }^{9}$, it would be of interest to verify if the blockade of $\alpha_{2}$-adrenergic heteroreceptors on 5 -HT fibers could potentiate the enhancing effect of 5-HT terminal autoreceptor antagonists. Methiothepin has the unique property, among various 
terminal 5-HT autoreceptor antagonists, of substantially increasing the evoked release of [3H] $3-\mathrm{HT}$ in basal conditions (without a SSRI), and this has been attributed to a possible inverse agonism of the drug at terminal 5-HT autoreceptors $^{21}$. It might be postulated, alternatively, that the propensity of methiothepin to increase the release of 5-HT in basal conditions is explained by the dual blockade of 5-HT terminal autoreceptors and $\alpha_{2}$-adrenergic heteroreceptors on 5-HT fibers as methiothepin was shown to be a potent $\alpha_{2}$ adrenoceptor antagonist ${ }^{11}$. In keeping with this view, the mere blockade of terminal autoreceptors in basal conditions would not be sufficie.t to produce a net increase in the release of 5-HT, because the momentary enhanced output of this neurotransmitter resulting from the blockade of autoreceptors would trigger an enhanced output of NA via $5-\mathrm{HT}_{3}$ receptors. This would subsequently lead to a negative feedback on the release of 5-HT

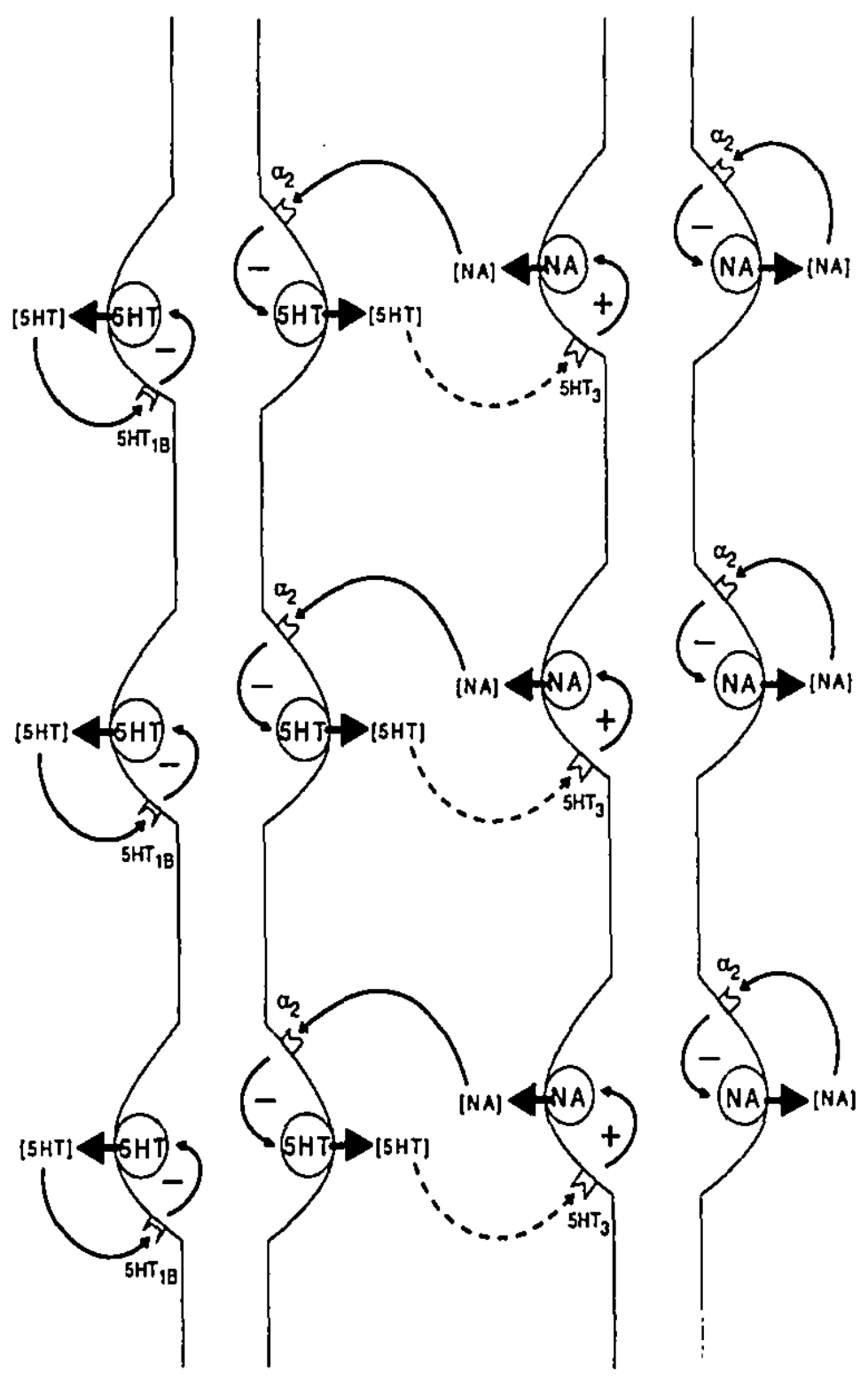

Fig. 1. Hypothetical regulatory loop involving $\alpha_{2}$-adrenergic and 5- $\mathrm{HT}_{3}$ heteroreceptors on 5-HT and NA varicosities. via $\alpha_{2}$-adrenergic heteroreceptors.

Another way to verify the hypothetical physiological relevance of a regulatory loop involving $\alpha_{2}$-adrenergic and 5-HT3 heteroreceptors would be to perform experiments following lesions of NA fibers. We could verify whether the effect of increasing the frequency of nerve terminal depolarization, by electrically stimulating either brain slices in vitro or the 5-HT pathway in vivo, would decrease the release of 5-HT in NA-denervated rats as much as in controls. The above hypothesis predicts that noradrenergic denervation would impair 5-HT autoinhibition 
mostly at high frequencies of terminal depolarization, i.e., when the biophase of 5-HT is high. So far, the only clue that we have comes from the second study (with befloxatone), in which it was shown that the efficacy of the stimulation of the 5-HT pathway, to suppress the firing activity of $\mathrm{CA} 3$ pyramidal neurons, is markedly enhanced four weeks after a pretreatment with 6-OHDA. However, these experiments would need to be reproduced with adequate control conditions before reaching a definite conclusion.

It could also be useful to determine if the inhibition of the evoked release of [3H]5-HT by exogenously applied 5-HT (in the presence of a SSRI) ${ }^{31}$ is altered by lesions of NA fibers. The end of the concentration-effect curve (high concentration of 5-HT) would be expected to be shifted to the right if a negative feedback mechanism mediated by NA is indeed important for 5-HT autoinhibition. A second topic of interest regarding the putative regulatory loop of figure 1 concerns the 5-HT3 receptors that enhance the release of 5-HT (see section 5.1). The electrically-evoked release of $[3 \mathrm{H}] 5-\mathrm{HT}$ was found enhanced when the SSRI paroxetine was introduced 8 minutes before stimulating preloaded brain slices. This enhancement in the release of [3H] $5-\mathrm{HT}$, produced by endogenous $5-\mathrm{HT}$, was markedly diminished when paroxetine was introduced 20 minutes instead of 8 minutes before the second stimulation. It was thus suggested that the 5-HT3 receptors mediating this enhancementrapidly desensitize through some intracellular mechanism ${ }^{3}$. An alternative explanation could be that a prolonged activation by paroxetine of the 5-HT3 receptors that enhance the release of NA (for 20 minutes, but not for 8 minutes) sufficiently enhances the inhibitory input at $\alpha_{2}$-adrenergic heteroreceptors, and thus counteracts the facilitarory action of the 5-HT3 receptors that enhance the release of [3H]5-HT. This appears possible inasmuch as the 5-HT3 receptors that enhance the release of NA do not rapidly desensitize (fourth article). It would thus be of interest to determine if the time-dependent decrease in the enhancing effect of paroxetine on the release of $[3 \mathrm{H}] 5-\mathrm{HT}$ could be prevented by lesions of the NA fibers.

The discovery that endogenous NA exerts a tonic inhibitory control over the release of 5HT via $\alpha_{2}$-adrenergic heteroreceptors in vivo provides an important insight into the behavioral physiology of the NA and 5-HT systems. Indeed, it can be inferred that any variation in the NA output will be reflected by parallel changes at the level of 5-HT output. As discussed earlier (section 3.2), LC NA neurons increase their firing activity in response to noxious, stressful or anxiogenic stimuli, resulting in an increased NA output in terminal brain areas. It can be assumed that noradrenergic responses to environmental stimuli will, in turn, decrease the release 5-HT via the activation of $\alpha_{2}$-adrenergic heteroreceptors on 5 -HT terminals. The release of NA in forebrain areas is thought to be important in the mediation of the facilitatory effects of arousal 
on various neuronal functions. For example, infused or synaptically released NA in rat hippocampus have been shown to increase the exploration of a novel environment, and increase hippocampal cells' responses associated with food rewards $10,27,28$. Increased NA neurotransmission also facilitates the acquisition of memory traces ${ }^{15}$. On the other hand, a particularly strong case was made for an inhibitory role of 5-HT on a variety of behavioral responses. Indeed, evidence was provided indicating that low levels of 5-HT are associated with a trend towards impulsivity and exaggerated stimulus reactivity 30 . 5-HT has been described as a neurotransmitter selectively disengaging memory traces ${ }^{14}$. Furthermore, Gray has reviewed findings ${ }^{13}$ supporting the notion that increases in 5-HT neurotransmission facilitate the activity of the septo-hippocampal system to suppress behavioral responses leading to punishment. It might thus be argued that the NA and 5-HT systems have antagonistic actions on some behavioral responses to the environment. In this regard, the tonic inhibitory input of endogenous NA at $\alpha_{2-}$ adrenergic heteroreceptors might render the NA system unable to exert a continuous control over the 5-HT behavioral inhibition system.

One salient biological correlate of the behavioral deficits produced by uncontrollable chronic aversive events (e.g. foot-shocks) in the learned helplessness model of depression is the depletion of NA in brain terminal areas. This phenomenon appears to have some psychological ground because no depletions of NA are produced when chronic stresses, of equivalent duration and intensity, are delivered to animals that are allowed to control the termination of the aversive stimuli (e.g. by pressing a lever) ${ }^{33}$. One consequence of the depletion of NA would be to increase the sensitivity of adrenergic receptors ${ }^{16}$. We can suppose that this could also occur in human depression as it was consistently found that high affinity $\alpha_{2}$-adrenoceptors are up-regulated in platelets of depressive patients (for a review see ${ }^{24}$ ). Previous findings ${ }^{2}$ and the results of our first. and second article with the denervation of the NA fibers with 6-OHDA suggest that the sensitivity of the $\alpha_{2}$-adrenergic heteroreceptors is increased by a sustained NA depletion. Based on these facts, it can be hypothesized that one etiological factor leading to depression is an up-regulation of $\alpha_{2}$-adrenergic heteroreceptors on 5-HT fibers. If this interpretation is correct, an outburst of NA in response to aversive events would decrease 5-HT neurotransmission in depressed patients more than in healthy individuals because of the supersensitivity of $\alpha_{2}$-adrenergic heteroreceptors. An indirect way to test this hypothesis would be to assess the responsiveness of $\alpha_{2}$-adrenergic heteroreceptors to intravenous clonidine in rats with the behavioral deficits of learned helplessness. 
The results of the present thesis provided evidence indicating that the interactions between the 5-HT and NA systems of the hippocampus are modified by long-term antidepressant treatments. It is of interest at this point to integrate these findings with those of the previous studies reviewed earlier (sections 7 and 8). Selective NA reuptake inhibitors have been studied for their ability to increase the synaptic concentration of NA. In the second article, long-term treatment with nisoxetine was shown to desensitize the $\alpha 2$-adrenergic heteroreceptors on 5-HT fibers (the effect of the high dose of clonidine was abolished). Although the inhibitory effect of high NA output appeared blunted by this treatment, the tonic inhibitory action of basal levels of endogenous NA on 5-HT neurotransmission was still present (unchanged effect of the small dose of clonidine). The sketches of figure 2 illustrate these notions: (1) Normally, 5-HT

(1) PRIOR TO TREATMENT
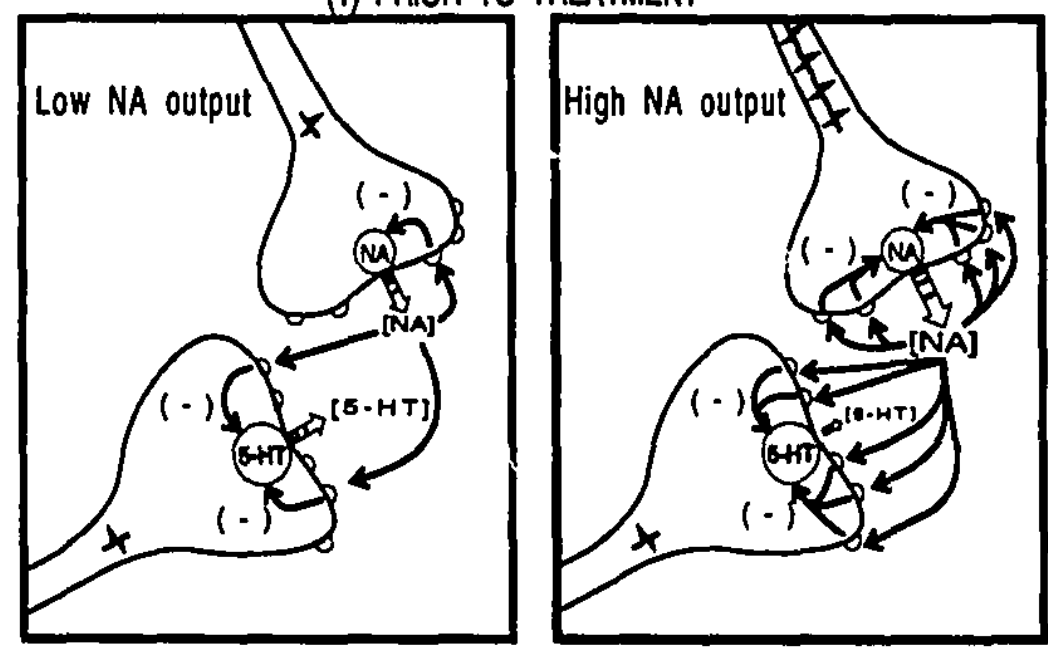

(2) FOLLOWNG LONG-TERM TREATMENT WITH NISOXETINE
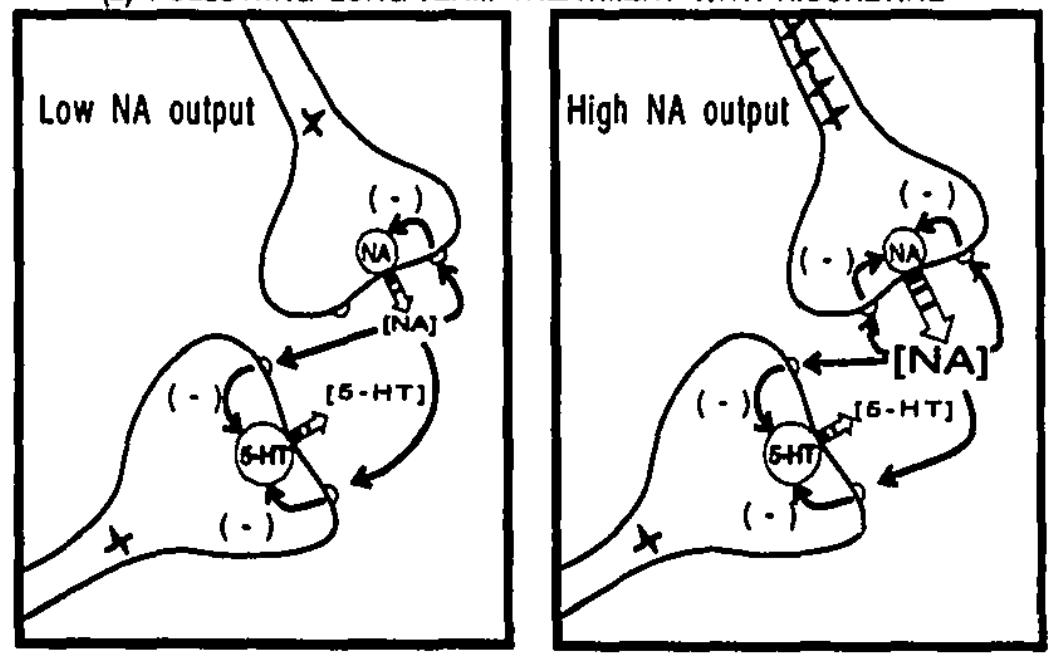

Fig. 2. Desensitization by nisoxetine of $\alpha_{2}$-adrenergic heteroreceptors on 5-HT terminals 
neurotransmission is decreased in the condition of increased NA output. (2) Following long-term treatments with nisoxetine there might be, for example, a down-regulation of $\alpha_{2}$-adrenergic heteroreceptors on 5-HT filers. The consequent desensitization of the $\alpha_{2}$-adrenergic heteroreceptors would not hinder the inhibitory action of low NA output. However, an increased NA output would not decrease further the release of 5-HT. The consequence of all this is that 5HT neurotransmission would remain under the tonic control of the NA system after long-term treatments with antidepressant drugs that block NA reuptake. However, the decline in the release of 5-HT produced by outburst of NA, in reaction to aversive events for instance. would be attenuated.

In contrast, it is difficult to make any assumptions regarding the status of the NA neurotransmission after long-term inhibition of the NA reuptake carrier. Some studies, but not all, found that these treatments decrease the firing activity of LC NA neurons (section 7.1), thus suggesting that they produce a decreased NA output. The opposite conclusion can be reached, however, considering that the evoked release of [3H]NA (fifth article) and the efficacy of the stimulation of the NA pathway (at $5 \mathrm{~Hz}$ ) to suppress the firing activity of $\mathrm{CA}_{3}$ pyramidal neurons ${ }^{17}$ were both found increased following long-term treatments with desipramine. But this contrasts with the effect of the long-term maprotiline treatment which did not change the evoked release of [3H]NA (fifth article). There are also contradictions regarding the sensitivity of the terminal $\alpha_{2}$-adrenergic autoreceptors after long-term NA reuptake inhibitors. Administration of high doses of desipramine was shown to desensitize these autoreceptors when assessed in vivo ${ }^{17}$, but not in vitro 8,26 . Finally, we have shown that the $5-\mathrm{HT}_{3}$ receptors that enhance the release of NA become desensitized following long-term treatments with desipramine, but not with maprotiline (fifth article). It was postulated that this might be a homoeostatic mechanism that restrains 5-HT3 receptors to enhance the release of NA when inis parameter is already very high.

We have also studied the effect of antidepressant drugs that block selectively the 5-HT reuptake carrier. Long-term treatments with SSRIs have been shown to desensitize both somatodendritic and terminal 5-HT autoreceptors. Indeed, short-term treatments with SSRIs decrease the firing rate of DR 5-HT neurons. However, this firing activity progressively returns to baseline level in the course of long-term treatments as the somatodendritic 5-HT/A autoreceptors become desensitized (section 7.4). Moreover, the amount of 5-HT released per each action potential is enhanced consequently to the desensitization of terminal 5-HT autoreceptors (section 8.1.4). These effects would account for the enhancement of 5-HT neurotransmission following long-term SSRIs. In the second article, we have seen that long-term treatment with paroxetine 
does not change the sensitivity of $\alpha_{2}$-adrenergic heteroreceptors, thus suggesting that these receptors are not involved in the therapeutic effect of SSRIs. Nonetheless, we have discussed earlier (section 9.1) the results of Blier et al. ${ }^{6}$ indicating that the occupation of the 5-HT reuptake carrier, and the consequent activation of terminal 5-HT autoreceptors, decreases the sensitivity of $\alpha 2$-adrenergic heteroreceptors through some molecular link. This decreased sensitivity of $\alpha_{2}$ adrenergic heteroreceptors was not observed here (second article) because there was no paroxetine present in the brain of the animal at the moment of the experiments (the minipumps containing the drug were removed two days before). In future electrophysiological experiments, we could determine if the inhibitory effect of high intravenous dose of clonidine is attenuated in the presence of paroxetine. After all, it is possible that this acute effect of SSRIs contributes, with the desensitization of 5-HT autoreceptors, to increase 5-HT neurotransmission.

Contrary to $\alpha_{2}$-adrenergic heteroreceptors, the 5 -HT3 receptors that enhance the release of NA did not become desensitized following long-term treatments with a selective reuptake blocker that increase the synaptic concentration of the endogenous neurotransmitter acting at the receptor (fifth article). It can thus be assumed that elevation of 5-HT concentrations can be normally translated into increased release of NA via the activation 5-HT3 receptors after longterm treatments with SSRIs. The expression of tyrosine hydroxylase was found to be increased in the LC area following long-term treatment with fluoxetine 7 . Interestingly, the evoked release of [3H]NA was also increased in rats treated with this drug (fifth article). It was hypothesized that this is the result of an increased 5-HT input at the 5-HT3 receptors that enhance the release of NA, because in a previous study ${ }^{4}$ long-term treatments with a SSRI was shown to increase the evoked release of [3H]5-HT two days after the last administration of the drug. One way to test this hypothesis would be to introduce a 5-HT3 antagonist before $S_{1}$ and to compare the evoked release of [3H]NA from preloaded hippocampal slices of rats treated with fluoxetine for three weeks with that of saline-treated rats.

It was then of interest to consider the effect of long-term treatments with type A MAOIs as these antidepressant drugs increase the synaptic concentration of both NA and 5-HT. Although the firing rate of DR 5-HT neurons is decreased after short-term treatments with type A MAOIs, there is a gradual recovery of the firing activity of 5-HT neurons in the course of long-term MAOI treatments as the somatodendritic 5-HT 1 a autoreceptors become desensitized (section 7.3). This effect most likely contributes to increase 5-HT neurotransmission. Another presynaptic effect of MAOIs that could produce the same result, i.e. enhancement of 5-HT neurotransmission, is the increased release of 5-HT at the level of terminals. This increased 5-HT output cannot be explained by the desensitization of the somatodendritic 5-HT1A autoreceptors (the method used 
to assess their sensitivity bypasses the influence of raphe nuclei neurons) nor by a desensitization of terminal 5-HT autoreceptors (section 8.1.3). The results of the second study indicate that a long-term treatment with the MAOI befloxatone decreases the sensitivity of $\alpha_{2}$-adrenergic heteroreceptors. As was suggested ${ }^{4}$, the increased release of 5-HT observed after long-term MAOIs might be attributed to an attenuated sensitivity of $\alpha 2$-adrenergic heteroreceptors.

In contrast to DR 5-HT neurons, the firing activity of LC NA neurons remained inhibite. throughout long-term treatments with MAOIs, and there was no desensitization of either somatodendritic or terminal $\alpha_{2}$-adrenergic autoreceptors. In addition, the efficacy of the stimulation of the NA pathway to suppress the firing activity of $\mathrm{CA} 3$ pyramidal neurons was also found unaltered (sections 7.3 and 8.1.3). From these studies it could be concluded that the decreased firing activity of NA neurons produced a decrease in NA output at the level of the hippocampus. However, it was found (fifth article) that long-term treatment with the MAOI moclobemide increases the evoked release of [3H]NA in the hippocampus. It thus appears that some presynaptic mechanisms, not related to a desensitization of $\alpha 2$-adrenergic autoreceptors, contribute to increase NA neurotransmission. This might be attributed to an increased 5-HT input at the 5-HT3 receptors that enhance the release of NA inasmuch as the sensitivity of these receptors was found unaltered after long-term moclobemide treatments (fifth article). Overall, these results suggest that after long-term MAOI treatments the decreased firing activity of NA neurons is counteracted by an increased release per action potential of NA from terminals.

We have seen that long-term TCAs and ECS do not generally have prominent effects at the level of the presynaptic 5-HT receptors that control the firing rate of DR 5-HT neurons nor those that modulate the release of $5-\mathrm{HT}$ in terminal areas (sections 7.1, 7.2, 8.1.1, 8.1.2). It was suggested that these treatments increase 5-HT neurotransmission by their long-term action on postsynaptic 5-HT receptors. For instance, many investigations (sections 8.1.1 and 8.1.2) as well as our present results (third article) indicate that the sensitivity of 5-HT $1 \mathrm{~A}$ receptors that inhibit the firing activity of $\mathrm{CA} 3$ pyramidal neurons is increased by long-term treatments with TCAs and by ECS. Furthermore, the fact that the sensitivity of $\alpha 2$-adrenergic heteroreceptors was not changed by ECS (third article) brings forth another argument indicating that the increased 5-HT neurotransmission produced by this type of treatment does not involve presynaptic receptors on 5-HT neurons.

Several indications would suggest that another prominent effect of TCAs and ECS is to decrease NA neurotransmission in the hippocampus. Although the results remain controversial, some studies indicated that the firing activity of LCNA neurons is decreased by long-term TCAs. Second, the expression of the synthesizing enzyme of NA, tyrosine hydroxylase, is decreased by 
ECS and by TCA treatments (section 7.1 and 7.2). Third, $\beta$-adrenergic functions and receptor number are also decreased by these treatments. We have also learned that long-term treatments with TCAs that have either weak or no NA reuptake potency, such as trimipramine, do not change the amount of NA released from terminals nor the sensitivity of the 5-HT3 receptors that enhance the release of NA (fifth article). But the picture is complicated by the fact that desipramine, a high potency NA reuptake blocker, produces an important enhancement in the evoked release of NA. Nevertheless, it might be argued that there is no net increase in NA neurotransmission after long-term treatments with this drug since it desensitized the 5 - $\mathrm{HT} 3$ receptors that enhance the release of NA in addition to the $\beta$-adrenoceptor:

Despite the fact that the sensitivity of 5-HT3 and $\alpha_{2}$-adrenergic heteroreceptors was not changed by the TCA trimipramine and ECS, it is important to emphasize that there is evidence indicating that interactions between the NA and 5-HT systems are important in the mechanism of action of these types of antidepressant treatments. For example, the sensitization of the $5-\mathrm{HT}_{1 \mathrm{~A}}$ receptors that inhibit the firing rate of $\mathrm{CA} 3$ pyramidal neurons by long-term TCA treatments was prevented by lesions of the noradrenergic neurons ${ }^{12}$. Furthermore, the desensitization by longterm desipramine treatments of the $\beta$-adrenergic mediated production of cAMP in the cerebral cortex was prevented by lesions of the serotonergic fibers ${ }^{1}$.

It was also of interest to consider the effects of some at;pical intidepressant drugs. Longterm treatments with mianserin and idazoxan share with classical noradrenergic antidepressant drugs (inhibitors of $\mathrm{MAO}$ and NA reuptake) the ability to desensitize $\alpha 2$-adrenergic heteroreceptors (third article). This desensitization is most likely explained by an increased synaptic concentration of NA during the treatment, resulting from the blockade of $\alpha_{2}$-adrenergic autoreceptors (sections 7.5 and 8.1.5). Interestingly, the main metabolite of antidepressant drugs of the azapirone family, 1-PP, blocks also $\alpha_{2}$-adrenergic autoreceptors (sections 7.5 and 8.1.5). Therefore, long-term treatments with azapirones should theoretically desensitize $\alpha_{2}$-adrenergic heteroreceptors like idazoxan and mianserin did. It would be of interest to verify this assumption using the same electrophysiological paradigm with clonidine. Azapirones, similarly to SSRIs and MAOIs, were shown to desensitize 5-HT $1 \mathrm{~A}$ autoreceptors on 5-HT neurons (section 7.5), and the 5-HT1A agonist activity of these agents may increase the serotonergic input at postsynaptic 5HTIA receptors since they do not desensitize these receptors (section 8.2.1). It is possible that the latter two properties, in addition to the desensitization of $\alpha_{2}$-adrenergic heteroreceptors, act together to increase 5-HT neurotransmission after long-term treatments with azapirone antidepressant drugs. 
The status of the NA neurotransmission after these treatments can be debated from the results presently available. The enhancing effect of the small dose of clonidine that is mediated by $\alpha_{2}$-adrenergic autoreceptors was markedly reduced after the idazoxan and mianserin treatments (third article). Studies which have directly assessed the function of terminal $\alpha_{2}-$ adrenergic autoreceptors after long-term idazoxan and mianserin treatments revealed that these receptors become supersensitive in response to their sustained blockade (section 8.1.5). It was concluded that the amount of NA released per action potential is decreased after treatments with antidepressant drugs that block $\alpha_{2}$-adrenoceptors. The NA input at postsynaptic targets, such as the $\alpha_{2}$-adrenergic heteroreceptors, should consequently be decreased (third article). Furthermore, the sensitivity of postsynaptic $\beta$-adrenoceptors was shown to be decreased by long-term mianserin treatments (section 8.2.2). Nevertheless, it is not clear if the net effect of these agents is to decrease NA neurotransmission considering that, during treatment, these agents block $\alpha_{2}-$ adrenergic autoreceptors and should therefore maintain an elevated NA output.

The evidence gathered in the present thesis could be useful for the development of new pharmacological strategies for the treatment of depression. It was recently shown that depressive patients respond more rapidly to medication when they are treated with the combination of a selective NA reuptake inhibitor plus a SSRI than with either drug alone ${ }^{23,29}$. Yet, antidepressant drugs that either block NA or 5-HT reuptake are equaliy effective in the treatment of depression 35 . It could be argued from these data that an antidepressant drug that desensitizes the $\alpha 2$-adrenergic heteroreceptors on 5-HT fibers, by blocking NA reuptake, as well as the terminal 5-HT autoreceptors, by blocking 5-HT reuptake, is a superior strategy for a rapid treatment of depression. Although MAOIs increase the synaptic concentration of both NA and 5-HT and desensitize $\alpha_{2}$-adrenergic heteroreceptors, treatments with these drugs would not produce rapid antidepressant responses because they do not desensitize terminal 5-HT autoreceptors ${ }^{5}$. It will be of interest to follow up the clinical trials with compounds such as duloxetine and venlafaxine, which potently block both NA and 5-HT reuptake 22,34 , as data suggest that these drugs may exert a more rapid and robust antidepressant response 25,32 . Another strategy could be to use a potent $\alpha 2$-adrenergic autoreceptor antagonist as an adjunctive therapy to a SSRI treatment. Idazoxan, for instance, would be a treatment of choice as this drug is as effective as a NA reuptake blocker to desensitize $\alpha_{2}$-adrenergic heteroreceptors on 5-HT fibers.

The effects of repeated administration of 5-HT3 antagonists have been recently investigated in the learned helplessness paradigm of depression. Similarly to classical antidepressant drugs, administration of low doses of ondansetron, zacopride or tropisetron reduced the behavioral deficits produced by uncontrollable foot-shocks ${ }^{19}$. It is possible that the 5-HT3 
receptors that enhance the release of NA are implicated in this "antidepressant" effect. The reversal of helplessness behaviors by antidepressant drugs is thought to be related to an increase in 5-HT neurotransmission 20 . In this context, it might be argued that by blocking 5-HT3 receptors, the purported negative feeback mediated by these receptors and the $\alpha_{2}$-adrenergic heteroreceptors (fig. 1) is impaired and therefore allows a greater release of 5-HT. However, this is highly speculative, and data indicating that 5-HT3 antagonists are effective antidepressive agents in humans are awaited before exploring further this possibility.

Overall, our results suggest that there are interactions between the NA and 5-HT systems in terminal areas such as the hippocampus, and that these are altered by some, but not all, antidepressant treatments. Nonetheless, a global picture of the NA - 5-HT interactions in the whole brain can hardly be outlined at the present time because there are no data concerning the relative contribution of similar interactions in other brain areas. It was mentionned, for instance, that there are $\alpha$-adrenoceptors and 5-HT receptors that modulate the firing activity of DR and LC neurons (section 9.4). We do not know whether these receptors or those in terminal areas are predominantly affected by the administration of noradrenergic and serotonergic agents. It would also be of interest to explore whether these receptors modulating cell firing are involved in the mechanism of action of antidepressant treatments. This might well be the subject of future investigations.

\section{References}

1. Barbaccia, M. L., N. Brunello, D. M. Chuang, and E. Costa. (1983) On the mode of action of imipramine: Relationship between serotonergic axon terminal function and down-regulation of $\beta$-adrenergic receptors. Neuropharmacology 22:373.

2. Benkirane, S., S. Arbilla, and S. Z. Langer. (1985) Supersensitivity of $\alpha_{2}$-adrenoceptors modulating ${ }^{3} \mathrm{H}-5$ HT release after noradrenergic denervation with DSP4. Eur. J. Pharmacol. 119:131.

3. Blier, P. and C. Bouchard. (1993) Functional characterization of a 5-HT3 receptor which modulates the release of 5-HT in the guinea-pig brain. Br. J. Pharmacol. 108:13.

4. Blier, P. and C. Bouchard. (1994) Modulation of 5-HT release in the guinea pig brain following long-term administration of antidepressant drugs. Br. J. Pharmacol. 113:485.

5. Blier, P., Y. Chaput, and C. de Montigny. (1988) Long-term 5-HT reur tke blockade, but not monoamine oxidase inhibition, decreases the function of terminal 5-HT autoreceptors: an electrophysiological study in the rat brain. Naunyn-Schmiedeberg's Arch. Pharmacol. 337:246.

6. Blier, P., A. M. Galzin, and S. Z. Langer. (1990) Interaction between serotonin uptake inhibitors and $\alpha_{2}-$ adrenergic heteroreceptors in the rat hypothalamus. J. Pharmacol. Exp. Ther. 254:236. 
7. Brady, L. S., P. W. Gold, M. Herkenham, A. B. Lynn, and H. J. Whitfield,Jr.. (1992) The antidepressants fluoxetine, idazoxan and phenelzine alter corticotropin-releasing hormone and tyrosine hydroxylase mRNA levels in rat brain: therapeutic implications. Brain Res. 572:117.

8. Campbell, I. C. and R. M. McKernan. (1986) Clorgyline and desipramine alter the sensitivity of $\left[{ }^{3} \mathrm{H}\right]$ noradrenaline release to calcium but not to clonidine. Brain Res. 372:253.

9. Feuerstein, T. J., A. Lupp, and G. Hertting. (1987) The serotonin (5-HT) autoreceptor in the hippocampus of the rabbit: role of 5-HT biophase concentration. Neuropharmacology 26:1071.

10. Flicker, C. and M. A. Geyer. (1982) Behavior during hippocampal microinfusions. I. Norepinephrine and diversive exploration. Brain Res. Rev. 4:79.

11. Frankhuijzen, A. L. and A. H. Mulder. (1980) Noradrenaline inhibits depolarization-induced 3H-5-HT release from slices of rat hippocampus. Eur. J. Phormacol. 63:179.

12. Gravel, P. and C. de Montigny. (1987) Noradrenergic denervation prevents sensitization of rat forebrain neurons to serotonin by tricyclic antidepressant treatment. Synapse 1:233.

13. Gray, J. A. (1987) The neuropsychology of anxiety: An enquiry into the functions of the septohippocampal system. Oxford University Press, Oxford.

14. Handley, S. L. and J. W. McBlane. (1991) 5-HT-The disengaging transmitter. J. Psychopharmacol. $5: 322$.

15. Harley, C. W. (1987) A role for norepinephrine in arousal, emotion and leaning?: limbic modulation by norepinephrine and the Kety hypothesis. Prog. Neuropsychopharmacol. Biol. Psychiatry 11:419.

16. Johnson, J., A. Sherman, F. Petty, D. Taylor, and F. Henn. (1982) Receptor changes in learned helplessness. Soc. Neurosci. Abst. 8:392.

17. Lacroix, D., P. Blier, O. Curet, and C. de Montigny. (1991) Effects of long-term desipramine administration on noradrenergic neurotransmission reuptake: Electrophysiological studies in the rat brain. J. Pharmacol. Exp. Ther. 257:

18. Limberger, N., K. Starke, and E. A. Singer. (1990) Serotonin uptake blockers influence serotonin autoreceptors by increasing the biophase concentration of serotonin and not through a "molecular link". Naunyn-Schmiedeberg's Arch. Pharmacol. 342:363.

19. Martin, P., H. Gozlan, and A. J. Puech. (1992) 5- $\mathrm{HT}_{3}$ receptor antagonists reverse helpless behaviour in rats. Eur. J. Pharmacol. 212:73.

20. Martin, P., P. Soubrie, and A. J. Puech. (1990) Reversal of helpless behavior by serotonin uptake blockers in rats. Psychopharmacology 101:103.

21. Moret, C. and M. Briley. (1993) The unique effect of methiothepin on the terminal serotonin autoreceptor in the rat hypothalamus could be an example of inverse agonism. J. Psychopharmacol. 7:331.

22. Muth, E. A., J. T. Haskins, J. A. Moyer, G. E. Husbands, S. T. Nielsen, and E. B. Sigg. (1986) Antidepressant biochemical profile of the novel bicyclic compound $W y-45,030$, an ethyl cyclohexanol derivative. Biochem. Pharmacol. 35:4493. 
23. Nelson, J. C., C. M. Mazure, M. B. Bowers, and P. I. Jatlow. (1991) A preliminary, open study of the combination of fluoxetine and desipramine for rapid treatment of major depression. Arch. Gen. Psychiatry 48:303.

24. Pilez, J. E., A. Halaris, A. Saran, M.R. Marler (1991) Desipramine lowers tritiated paraaminoclonidine binding in platelets of depressed patients. Arch. Gen. Psychiatry 48:813.

25. Rickels, K. (1991) Venlafaxine: A new potent antidepressart agent with putative fast onset of action. Biol. Psychiatry 1:345.

26. Schoffelmeer, A. N. M. and A. H. Mulder. (1982) ${ }^{3}$ H-Noradrenaline and ${ }^{3} \mathrm{H}-5-\mathrm{HT}$ release from rat brain slices and its presynaptic $\alpha$-adrenergic modulation after long-term desipramine pretreatment. NaunynSchmiedeberg's Arch. Pharnuacol. 318:173.

27. Segal, M. and F. E. Bloom. (1976) The action of norepinephrine in the rat hippocampus. IV. The effects of locus coeruleus stimulation on evoked hippocampal unit activity. Brain Res. 107:513.

28. Segal, M. and F. E. Bloom. (1976) The action of norepinephrine in the rat hippocampus. III. Hippocampal cellular responses to locus coenuleus stimulation in the awake rat. Brain Res. 107:499.

29. Seth, R., J. Jennings, J. Bindman, J. Phillips, and K. Bergmann. (1992) Combination treatment with noradrenaline and serotonin reuptake inhibitors in resistant depression. Br. J. Psychiatry 161:562.

30. Spoont, M. R. (1992) Modulatory role of serotonin in neural information processing: Implications for human psychopathology. Psychological Bull. 112:330.

31. Starke, K., M. Gothert, and H. Kilbinger. (1989) Modulation of neurotransmitter release by presynaptic autoreceptors. Physiol. Rev. 69:864.

32. Tollefson, G. D., R. G. Thompson, J. H. Heiligenstein, S. James, D. T. Wong, D. E. Faries, and D. L. Hyslop. (1993) Is the potent, dual uptake inhibition of norepinephrine and serotonin by LY248686 (duloxetine) associated with a more rapid and robust antidepressant response? Am. Coll. Neuropsychopharmacology (Abstract)

33. Willner, P. (1985) Noradrenaline and depression: Theoritical perspectives. In Depression: $A$ psychobiological synthesis. Wiley, New York. 193-215.

34. Wong, D. T., F. P. Bymaster, A. M. Douglas, R. R. Leroy, J. H. Krushinski, and D. W. Robertson. (1993) LY248686, a new inhibitor of serotonin and norepinephrine uptake. Neuropharmacology 8:23.

35. Yazici, O., F. Aricioglu, G. Gürvit, A. Uçok, Y. Tastaban, . Canberk, M. Ozgüroglu, T. Durat, and D. Sahin. (1993) Noradrenergic and serotoninergic depression. J. Affect. Disord. 27:123. 